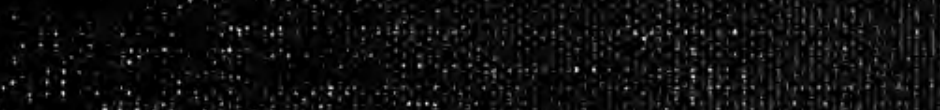

and

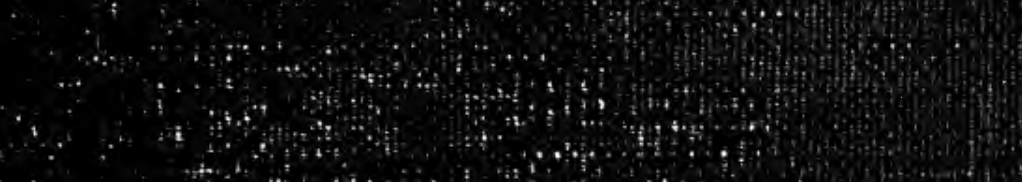
$\therefore$ as $\ldots$ 

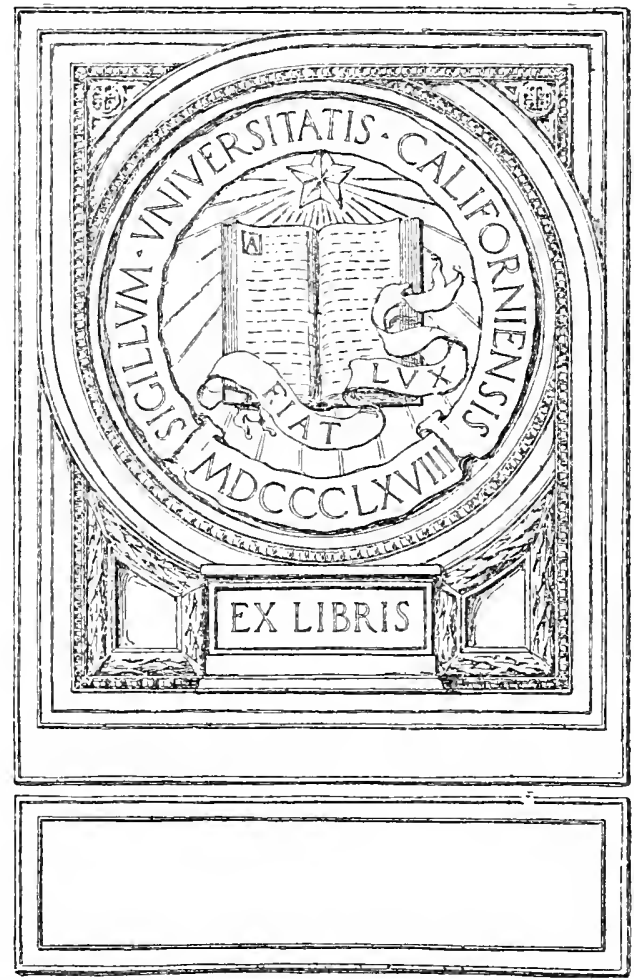


$$
\text { , }
$$




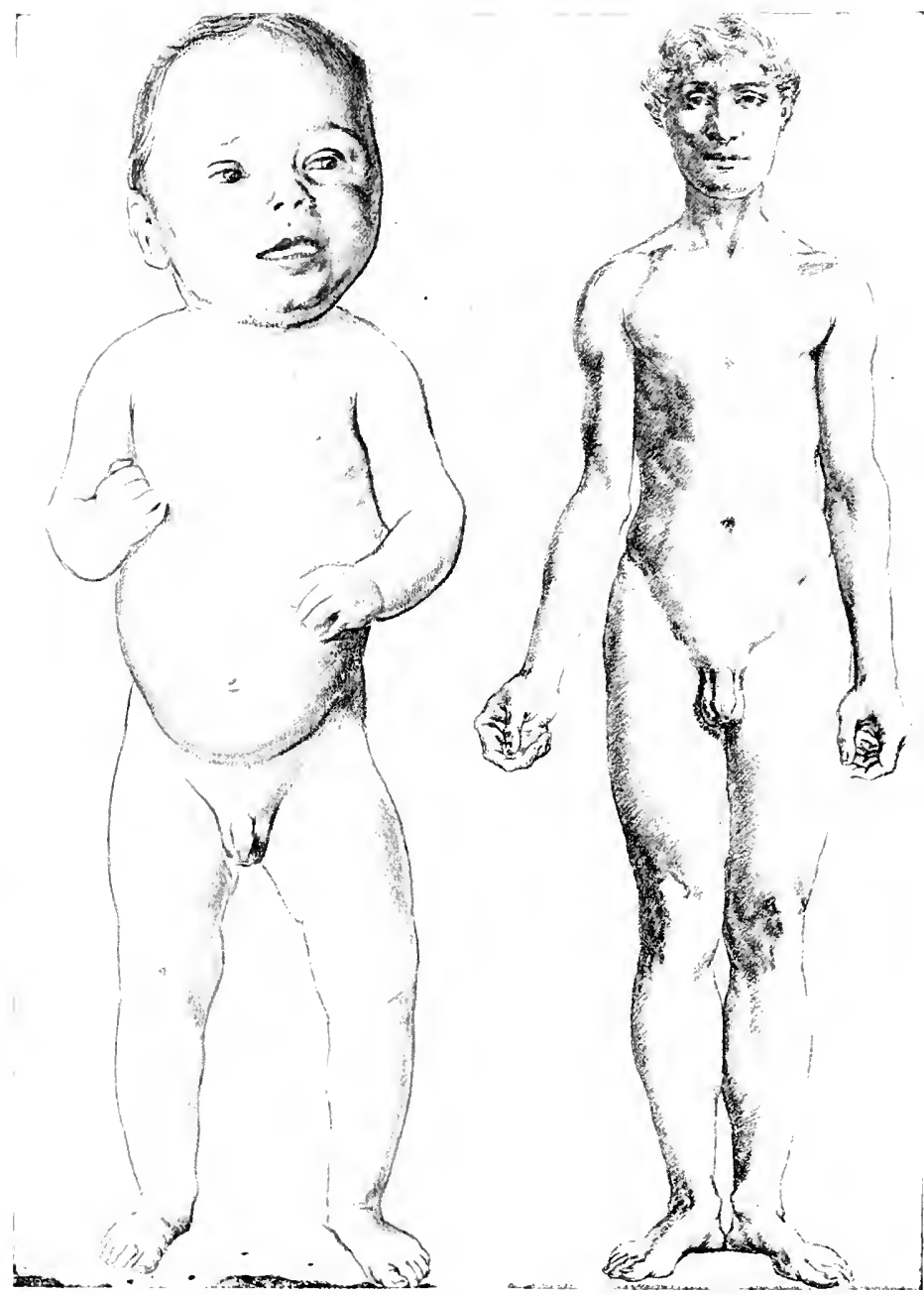

THE CHILD AND THE MAN.

In the Plate the figure of an infant of eleven months has been enlarged to equal in height that of an adult. The illustration shows at a glance the profound changes that are gradually effected during the years of growth. The infant's head is, relatively, large, but its arms and legs are short. Consequently the mid-point of the body, which is at the pubes in the adult, lies, in the infant, almost half-way up the abdomen. The slope of the shoulders, the form of the chest, the fulness of the abdomen, the development of the leg-muscles, and the shape of the feet are all strikingly different at the two ages. (See pp. 23 et seq.) 


\title{
CHILDREN IN HEALTH AND DISEASE
}

\author{
A STUDY OF CHILD-LIFE
}

\section{BY DAVID FORSYTH, M.D., D.Sc.}

PIYYSICIAN TO THE EVELINA HOSPITAL FOR SICK CHILDREN

assistant PHYSician (LATE PHYSICIAN in CHAROE OF THE CHILDREN'S DEPARTMANT) AND JOINT PAThologist, CHARING cross hospital

WITH FRONTISPIECE

\section{LONDON}

JOHN MURRAY， ALBEMARLE STREET, W. 
GEAERL 
MY FRIEND AND TEACHER

SIR COOPER PERRY 



\section{PREFACE}

ONE of the most notable developments of public opinion in recent years has been the growth of a widespreading interest in the conditions of child-life. This movement has specially concerned itself with the health of the young, which, for the first time, has become recognised as the essential factor in their lives. As a natural result, the direct bearing of medical science on all matters relating to childhood is now admitted. For the future, therefore, medical men will require a broader experience of children than can be obtained in the sickroom alone, and they must possess a more general knowledge of childhood than is implied in an acquaintance with its diseases.

While modern opinion has thus brought the physician into closer relation with childhood, it demands also that educationalists themselves should no longer regard the health of their scholars as a matter outside their province. They must be familiar with the physiology and psychology of children, and they should possess some knowledge of the medical problems of children in general and of school children in particular. The teacher and the doctor must meet on common ground. Both work for the same end, and, though they advance from different directions, each should attempt to understand the aims and difficulties of the other. The closer the harmony between them the better for the children.

In all cases, however, the final results of their efforts must largely depend on the attitude of those who, in municipal and public life, control the fate of the younger generation. Public authorities are not yet sufficiently alive to the lamentable amount of preventable disease that exists among children, nor do they fully appreciate the necessity of basing our educational 
methods on the physiological and psychological requirements of the young.

For these reasons I believe that a book dealing with childlife from a scientific standpoint is needed at the present time. The many works that have already been published are concerned, as a rule, only with some one or other aspect of childhood-its education, schools, diseases, and so on-and I know of none that presents a comprehensive account of the whole subject. This gap I have attempted to fill in the following pages by providing as complete a study of children as could be made within convenient limits. I realise only too well how far the result falls short of what I hoped to make it, and what others, more fitted for the task than myself, would have made it. Yet in a book such as this, which, though of limited size, covers a very wide field, the difficulty must always be to present a concise account of each subject without sacrificing clearness of outline or proportion. Readers who are interested in some particular branch of child-study may consider that I have treated their own specialty in insufficient detail. These I would ask to remember that we cannot obtain a true and sympathetic understanding of children from the standpoint of any one specialism alone. We shall see things in their proper perspective only when we take up our position at the meeting-point of the many sciences that are concerned in the development and welfare of the young.

This is the outlook I have endeavoured to present-always, however, keeping in the foreground the prime factors of health and disease. I have consistently borne in mind the greater importance of contrasting juvenile and adult qualities than of pointing out their resemblances. This fact is responsible for the slight attention which I have given to pathological processes in the young.

Although I have addressed myself primarily to medical readers, many of the subjects I have dealt with are of equal importance to teachers and others, some of whom may, however, retain the belief that the doctor is a disturbing influence in school life. I shall be more than satisfied if my book succeeds in convincing them that childhood offers the most promising 
field for the labours of the physician no less than those of the educationalist and the publicist, and that, for the successful co-operation of their different activities, it is only necessary to keep in mind the motive that they share in common-the wellbeing of the coming generation.

Before concluding, I am glad to have the opportunity of acknowledging $\mathrm{my}$ indebtedness to the writings of Dukes, Goodhart and Still, Ireland, Newman, Newsholme and Pakes, Pfaundler and Schlossmann, Preyer, Romanes and Spencer. In addition, I have availed myself of many official publications, including the annual reports of the Medical Officer (Education) to the London County Council, the report of the Royal Commission on the Care and Control of the Feeble-minded, the reports of the second International Congress on School Hygiene and of the first International Moral Education Congress, the annual reports of the Registrar-General, and numerous reports and articles in the Lancet and British Medical Journal.

Finally, I must thank my brother, Cecil Forsyth, for the many hours he has given to reading and criticising the proofsheets. His suggestions have been invaluable to me.

LONDON, W.

DAVID Forsyth. 



\section{CONTENTS}

\section{CHAPTER I}

\section{THE PHYSIOLOGY OF CHILDREN}

Introduction. Antenatal Physiology - The Phylogenetic Influence-The Influence of the Intra-uterine Environment: The Protection of the Embryo; The Effect of Gravity; Malformations; Amniotic Adhesions; Anencephalus; Acardiacus-The Influence of the Placenta: The Functions of the Placenta; Disease in the Mother; Bacterial Infections; Alcoholism; Disease in the Father-The Physiology of the Fœtal Child at Term ; Inactive Organs; The Lungs, etc. ; Active Organs ; The Heart, Liver, etc. Natal Physiology-The Influence of the Mechanical Forces of Parturition; Physiological Changes at Birth ; Respiration ; Negative Intrathoracic Pressure-Changes in the Heart .

\section{CHAPTER II}

\section{THE PHYSIOLOGY OF CHILDREN (concluded)}

Postnatal Physiology. Physiology of Growth-Interdependence of Organs; Changes in the Skeleton and in Bodily Proportions ; Growth in Height ; Growth in Weight ; Growth of Individual Organs. Physiology of CERTAIN SPECIAL FunCTIONS-Digestion; Circulation and Respiration; Body-weight and Surface-area ; Kidneys ; Sweat ; Carbon Dioxide ; Respiratory Exchange; Lymphoid Tissue. Physiology of METABOLISM-Experimental Methods; Growth and Animal Heat; Heat-Regulation; Its Development ; Nutrition; Caloric Requirements of an Infant; Caloric Values of Food-stuffs; Respiratory Quotient; Growth and Heat-Production; Age not the Criterion of Food Requirements; Surface-area per kilo. body-weight; Principles of Infant Feeding; Total Heat-loss; Total Minimum of Energy required; Human Milk-Its Calorific Value; The Quantity Required ; Cow's Milk-Its Calorific Value; The Quantity Required; Influence of Muscle-work; Of Atmospheric Temperature; Size and Frequency of Feeds; The

Feeding of Older Children. PUbERTY 


\section{CHAPTER III}

\section{THE PSYCHOLOGY OF CHILDREN}

InTRODUCTION-Observations on Infant Psychology; Sensations the Basis of Mentality ; Ideas ; Recognition ; Memory ; Judgments ; Influence of Environment; Walking; Talking. AT BIRTHSleep ; Hunger ; Pain ; Hearing ; Sight ; No Intelligence ; No Emotions; No Power of Expression; Automatic and Reflex Muscular Movements. THE FIRST MONTH-Sleep; Impulsive Movements; Yawning; Hiccoughing; Movements of Eyes and Eyelids; Taste and Smell; Effect of Light; Appreciation of Sound ; Pleasant and Unpleasant Feelings ; Smiling ; Phonation ; Mental Association. THE SECOND, THIRD, AND FOURTH MONTHS-Reflex Movements ; Co-ordination of Eyes ; Balancing Head ; Sensations ; Grasping Objects Seen ; Hearing ; Emotions ; Fear of Strangers. The FifTh, SiXTh, AND Seventh Months -Use of Arms; Interest in Toes; Grasping Movements; Muscular Sense; Sitting Posture; Pleasure from Auditory Sensations; Expression of Feeling; Recognition; Memory; Early Consciousness of Self

\section{CHAPTER IV}

\section{THE PSYCHOLOGY OF CHILDREN (concluded)}

The Eighth, Ninth, and Tenth Months-Instinctive Movements ; The Static Sense ; Judgments of Distance ; Capacities of a Child at Nine Months; Conscious Imitation; Attempts to Stand; Understanding of Words. THE END OF THE FIRST YEAR-Locomotion; Psychological Importance of Walking; Other Co-ordinated Movements; Curiosity; Stages in the Development of Speech; Intellect ; Judgments ; Reasoning ; Appreciations of Causality; Experimental Investigations by Infants; Repetition of Experiments ; Consciousness of Self. The SECOND Year-Locomotion; Climbing Stairs; Use of Joints; Speech; Monosyllabic Words; Mistakes in Use of Words; Consciousness of Self still Imperfect; The I-feeling; Intellectual Advances; Limited Experience responsible for Erroneous Judgments; Obedience ; Imagination. THE THIRD YEAR AND LATER-Complex Muscular Movements ; Skipping; Jumping ; Colour Sense: Emotions more Complex; Timidity; Sympathy; Affection; Ideas of Altruism; Use of the First Personal Pronoun; Imagination; Intellect 


\section{FYGIENE OF SCIIOOLS AND OF SCHOOL LIFE}

INTRODUCTION - Importance of School Hygiene; Parents and School Hygiene; Hygiene of Boarding Schools. HyGienE OF ScHOOLS-Boarding-Schools : Site, etc. ; Dormitories ; Internal Arrangements of Dormitories; Their Cubic Capacity; Their Furniture ; School Water-Supply ; Milk-Supply ; Laundry-Day Schools: Site, etc.; Classrooms; Size; Ventilation ; Artificial Heating; Natural and Artificial Illumination; Wall-colouring ; Furniture; School Desks. Hygiene of ScHOOL LIFE-Food : Cantines Scolaires of Paris; Food at Boarding-Schools; Times and Numbers of Meals; Alcohol; Hours of Sleep; Mental Fatigue; Physical Strain of Classroom Work; Intervals for Exercise; Daily Programme of Work; Number of Working Hours ; Home-lessons; Holidays ; Relation between Work and Play ; School Games; Clothing of School Children

\section{CHAPTER VI}

\section{MEDICAL ASPECTS OF SCHOOL LIFE}

InTRODUCTION-Hygienic Differences between Home-life and Schoollife. InFECTIOUS AND CONTAgious Diseases - Zymotic Diseases: Spread by Schools; Infectious Children attending Schools; Teachers Penalised if Infectious Children sent away from School ; Infectious Children and Prize Attendances ; Sunday Schools and the Spread of Infectious Fevers; Prevention of Spread of Infectious Fevers in Schools; Health Certificates; Schoolroom Cleanliness; Exclusion of Scholars; Closure of School; Closure of Infant Department; Closure for different Fevers - Infectious Diseases in Boarding-Schools - Tuberculous Diseases: Statistics of Tuberculous School Children; Infected Schoolrooms ; Infectious Scholars ; Infectious Teachers -Contagious Skin Diseases. CONDITIONS DEPENDENT ON Classroom Hygiene: Responsibility for Maintaining Healthy Conditions ; Training of Teachers in Elementary Hygiene and Physiology-Bad Ventilation and its Effects-Unsuitable Schooldesks-Injury to Sight from Unsuitable School-work-Overwork.

\section{CHAPTER VII}

\section{MEDICAL INSPECTION AND SUPERVISION OF SCHOOL, CHILDREN}

Duties of School Medical OfFicers : The Education (Administrative Provisions) Act, I9O7 ; The Board of Education and the Medical Inspection of Schools; Outline of the Scheme; Duties 
of the School Medical Officer; School Hygiene; Inspection of

Children; Routine Inspection and Detailed Examination; Prevention rather than Cure ; Frequency of Medical Attendance ; Trivial Febrile Attacks; Underfed Children; Half-timers. Methods OF InSPECTION : Medical Requirements ; Preliminary Assistance by School Nurse; Routine Inspection-General and Anthropometric-Eyes and Ears-Mental Capacity and Speech - Mouth and Throat ; Special Examination; Schedule of Medical Inspection; Annual Reports. Common Pathological CoNDITIONS OF SCHOOL CHILDREN-Dental Caries-Enlarged Tonsils and Adenoids-Swollen Glands in the Neck-Lateral Curvature of the Spine-Stammering and its Treatment. INVALID ScHOOLS : Their Object ; Children suitable for Admission; Open-air Schools. MEdical SUPERvision OF BOARDINGScHOOLS : Physique of Children of the Upper Classes; Unsatisfactory Hygienic Conditions of Boarding-Schools; School Sanatoria; Required Amount of Invalid Accommodation; Sickrooms; Attendance and Duties of Medical Officers; Preliminary Examination of Boys on first entering School .

\section{CHAPTER VIII}

\section{THE TRAINING OF CHILDREN}

Introduction. Educational Methods of the Present Day. THE MATERIAL: Animal Instincts; Habit-formation; Attention; Enthusiasm; Sensitiveness to Blame; Desire for Sympathy. ThE Methods: Methods must conform to the Process of Evolution; Stages in Training; (I) Spontaneous Training; Infant Education; Sensorial Training; Motor Training ; Infant "Play"; Habits of Life ; Speech ; (2) Disciplinary Training; Obedience; Prizes and Punishments; Religious and Esthetic Influences; Schools; Use of Leisure ; Relation between Home and School ; Minimum Age for School Attendance; Scholastic Instruction ; (3) Adolescence ; Relaxation of Authority ; Ideas of Altruism and of Community of Action; Intellectual Training ; Inductive and Deductive Reasoning; Puberty. The SPECIAL Training of GIRLS: Comparison between Girls and Boys from the point of view of their Training; Co-education of the Sexes : Rules of Health ; Phenomena of Sex; School Training; Domestic Economy; Child-rearing; Influence of Teachers on Girls. ABNoRMal TyPes OF CHILDREN: The Selfish; The Passionate; The Cruel; The Lying; The Precocious; The Neurotic

\section{CHAPTER IX}

\section{IDIOTS AND FEEBLE-MINDED CHILDREN}

Methods of Public Control: Historical; Guardians of the Poor; Metropolitan Asylums Board; Special Schools; Results of Training in Special Schools; Recommendations of the Royal 
Commission on the Care and Control of the Feeble-minded.

NUMBER OF MENTALLY DEFECTIVES in ENGLAND AND Wales. Causes of Mental Deficiency. Classification OF MENTAL DEFICIENCY: Idiots, Imbeciles, and Feebleminded ; Congenital Idiocy; Microcephalic ; Hydrocephalic ; Mongols ; Cretins; Primary Amaurotic; Genetous; Birth Palsies ; Acquired Idiocy; Epileptic ; Post-Febrile; Traumatic ; By Deprivation; General Remarks on Idiots. Diagnosis of IDIOCY IN INFANTS. TRAINING OF IDIOTS AND MENTALly Deficient ChILdReN : At Home and in Institutions; Combined Defects; Results of Training; General Care ; Moral Training; Physical Training; Sensory; Muscular; Speech ; Intellectual Training. MORAL INSANITY . . .

\section{CHAPTER $\mathrm{X}$}

\section{INFANT MORTALITY}

The Age-Factor in INFANT Mortality : The First Five Years ; The First Year; Infant Mortality, I85I-I907; General Deathrate; Birth-rate; Infant Mortality in each Quarter of the First Year; The First Quarter; The First Month ; The First Week; The First Day. THE Influence of Town Life : Urban and Rural Counties; Density of Population, etc. THE INFLuENCE OF ILLEGITIMACY. DISEASES RESPONSIBLE FOR INFANT Mortality : Wasting Diseases ; Diarrhcea ; Acute Pulmonary Diseases ; Convulsions ; Tubercle ; Syphilis; Rickets ; Infectious Fevers; Death Certification; Statistics of Infant Mortality in the Evelina Hospital for Sick Children, I885I906; Conclusions

\section{CHAPTER XI}

\section{THE CONDITIONS OF INFANT LIFE}

CONDITIONS AT BIRTH : Deaths in the First Month ; Injury at Birth ; Congenital Defects. IMMATURITY AS A CAUSE OF INFANT MoRTAlITy : Physical Deterioration; Industrial Employment of Women; Want of Food; Heredity; Notification of Births; Post-mortem Examination of Stillborn Infants; Legitimacy and Illegitimacy; Parental Neglect; Preventable Deaths. Conditions of LATER INFANT LifE: Infant Health, Good Food and Cleanliness; Maternal Neglect; Infant Life Assurance; Baby-farming ; Mothers who "go out"; Dirt ; Improper Feeding; Infantile Diarrhœa; Breast versus Bottle; Tuberculous Disease. THE REMEdies: Legal Remedies; Social Remedies; Infant Mortality merely one aspect of the problem of the Poor ; Education of Mothers ; Lessons in Babyrearing; Health Societies; Voluntary and Municipal Effort; Pure Milk Supplies ; Prohibition of Industrial Labour for Women after Confinement ; Day Nurseries. 


\section{CHAPTER XII \\ CHILDREN AND DISEASE}

INCIDENCE OF DISEASE IN CHILDHOOD: Factors diminishing the Incidence of Disease in Childhood; The Simplicity of Child-life ; Secondary Pathological Changes; Hereditary Diseases ; Sexual Instinct; Diseases incident to Parturition; Habits of Excess ; Occupational Diseases; Muscular Strain; Many Diseases of unknown Pathology restricted to Adult Life-Factors increasing the Incidence of Disease in Childhood ; Congenital Deformities; Injuries at Birth ; Susceptibility to Bacterial Infections ; Interference with Growth; Physiological Equilibrium of Childhood unstable; Certain Diseases restricted to Childhood; The Helplessness of the Young. PECULIARITIES OF CHILDREN IN DISEASE : Children dislike Illness; Neurotic Affections uncommon; Malingering; Chronic Illness; Loss of Muscular Activity; Mental Tiredness; Habit-spasms; Interference with Growth; Heart-disease; Appetite; Acute Illness ; Constitutional Symptoms prominent; Epileptic Convulsions; Cerebral Symptoms; Irregular Clinical Course; Rapidity of Recovery; Complications and Sequelæ frequent; Sudden Death .

PAGE

\section{CHAPTER XIII}

\section{ON EXAMINING INFANTS AND CHILDREN}

The Child's Fear of the Doctor ; Crying assists the Examination in some respects ; Position of the Patient ; Medical History ; Value of the Mother's Observations ; General Inspection of the Patient ; Painful Examinations; Risks in handling the Child, in impeding his Movements, in startling him; Auscultation; Procedure; Percussion; Abdominal Examination; Examination of the Nervous System; Rectal Examination; Examination of the Urine; Examination of the Blood; Temperature Records; Examination of the Pulse; Examination of the Sputum; Examination of the Vomit and the Fæces; Examination of the Tonsils

\section{CHAPTER XIV}

\section{THE DIAGNOSIS OF DISEASE IN CHILDREN}

General Considerations: Absence of Subjective Symptoms; Sex; Age; Season of Year; Prevalent Epidemics; Relative Frequency of Diseases. Diagnostic VAlue of Clinical SigNS AND Symptoms: General Appearance of the Patient; Importance of Minute Observation ; Diagnostic Value of Pain ; Condition of the Anterior Fontanelle; Weight and Growth; Deformities of Bones; Abdominal Examination; The Fauces; Temperature ; Pulse ; Respiration ; Expectoration ; Vomiting ; 
Constipation ; Diarrhœea ; Epistaxis ; Rigors. Diagnosis of

DiseASES OF THE HEART AND OF THE LUNGS: The Heart ; The Lungs; Peculiarities of the Healthy Vesicular Murmur; Auscultatory Signs of Pneumonia; Unequal Expansion of the Lungs; Transmission of Signs from one Lung to the other; Auscultatory Signs of Pleuritic Effusion . . .

\section{CHAPTER XV}

SOME POINTS IN THE TREATMENT OF SICK CHILDREN

Preventive Treatient: Cleanliness; Food; Sleep; Exercise ; Clothing. ACUTE Illness. Chronic Illness. Medicinal TREATMENT: Dosage ; Susceptibility to Drugs; Alcohol; Tonics; Pharmaceutical Preparations suitable to Children. SPECIAL REMEdial MEASURES: Methods inapplicable to Children; Baths; Antipyretic Remedies; Sponging, etc. ; Rectal Injections; Nutrient Enemata; Medicinal Enemata; Aperient Enemata ; Irrigation of Colon; Gastric Lavage ; Nasal Feeding. INFANT FEEDING: Feeding of Healthy Infants; Natural Feeding ; Wet-nursing ; Artificial Feeding ; Proprietary Foods ; Effects and Treatment of Improper Feeding; Wasting; Diluents of Milk; Gastro-intestinal Disturbances; Substitutes for Cow's Milk 



\title{
CHILDREN IN HEALTH AND DISEASE
}

\author{
CHAPTER I
}

THE PHYSIOLOGY OF CHILDREN

Introduction. Antenatal Physiology-The Phylogenetic InfluenceThe Influence of the Intra-uterine Environment: The Protection of the Embryo; The Effect of Gravity; Malformations ; Amniotic Adhesions ; Anencephalus; Acardiacus-The Influence of the Placenta: The Functions of the Placenta ; Disease in the Mother; Bacterial Infections; Alcoholism; Disease in the Father-The Physiology of the Fœtal Child at Term; Inactive Organs; The Lungs, etc.; Active Organs; The Heart, Liver, etc. NATAL Physiology-The Influence of the Mechanical Forces of Parturition; Physiological Changes at Birth; Respiration; Negative Intrathoracic Pressure-Changes in the Heart.

\section{INTRODUCTION}

GENERATIONS of parents, lacking the key to childhood, have attempted to apply to it the principles and the standards of adult life. They have sought to impose on one period of development the conditions which are suited only to a more advanced stage. To-day we are at last adopting a more rational attitude. We are beginning to understand that the child becomes the man by a process of gradual evolution, and that as the circumstances of early life are simple, so the conditions we impose at that time must be elementary. We are able to discern in the child's unshaped attributes, the faintly outlined characteristics of his future adult years; and, further, we are finding out that these attributes, if left to be moulded by nature alone, are likely to assume ugly forms. Our duty, there- 
fore, is to assist and guide the efforts of nature from the beginning, for the firmness and strength of the building can be assured only when the foundations have been deeply and truly laid.

In dealing with numbers of children, we soon find out that wide differences exist in their natures. These variations are by no means always restricted within the limits of normality; many children, indeed, are in some respect or another definitely abnormal. Yet their abnormalities are as unshaped as their normalities, and they can often be modelled to what shape we please. We must not, however, apply ordinary methods to extraordinary children. We must study very carefully the peculiarities of each child, and we must adapt our methods accordingly. As a result, we shall necessarily be drawn to a close examination of the child-mind, and gradually we shall learn that many of its variations are to be explained on strictly pathological grounds. This discovery will show us that a normal child is the product of physiological conditions; and thus we shall reach the conclusion that if we are to guide nature in her efforts to transform a child into a man, we must be well acquainted both with the physiological conditions of childhood, and with its more common pathological influences.

The full recognition of this supremely important fact will affect two changes in our attitude towards childhood. First, it will compel educationalists to alter their methods of training, and to bring them into harmony with modern physiological science. Second, it will widen the physician's share in the problems of childhood, and will bring him into intimate contact with methods of education and training. Hitherto he has interested himself merely in sick children. For the future he must realise his own direct concern in all the questions that deal with childhood; with the preservation of health in the individual and in the child-community no less than with the cure of disease; with class-room training and with moral education no less than with physical training. His special experience qualifies him to distinguish the healthy from the morbid, to recognise the slighter degrees of abnormality, to trace their causes, and to foresee their effects. And, since any 
mental or physical peculiarity in the child may easily determine a radical change, mental, moral, or physical, in the future man, it is vitally important that even slight defects should be ascertained and rectified early in life. A more extensive application of preventive measures will considerably diminish the number of children who grow up defective in body or in mind, and therefore will, in due time, effect an alteration in the conditions of the adult population.

In order to understand childhood, we must begin, then, with its physiology. But our study would necessarily be imperfect if we took up the subject only at the time when the child is already born. At this period he has, literally speaking, completed the greater part of the changes that occur between the germinal cell and old age. We must therefore give our first attention to antenatal life. Unfortunately this subject is one that is but little understood, and, amid innumerable speculations, established facts are rare. We do, however, know enough to assert that antenatal influences may be and often are deepreaching enough to modify the whole future life-history of the child. On this account, therefore, we must acquaint ourselves with the principal conditions that influence intra-uterine development. When we have considered these questions, there will be yet another period requiring our attention, before we turn to the conditions of postnatal life. The process of birth itself implies profound physiological changes in an infant, and these we must consider by themselves. It will be necessary, therefore, to deal with the whole subject under the three headings :-

\section{Antenatal Physiology. \\ 2. Natal Physiology. \\ 3. Postnatal Physiology.}

In making this division, we must be careful to remember that the development of children is not made up of sharply limited stages. From the time of conception onwards, the natural processes follow each other in an uninterrupted course, although at certain periods they are subjected to sudden or to extensive changes. 


\section{I.-Antenatal Physiology}

The science of antenatal physiology exists at present only in a rudimentary form, and the facts at our disposal are not sufficiently numerous to indicate more than a few elementary principles. We can, however, recognise three separate influences that modify the growth of the unborn child. First in point of time and of importance comes the phylogenetic influence, whereby the child, as the last in a long descent, is compelled to develop along certain definite lines. Next we have the influence of the strictly intra-uterine environment. Lastly we have the influence of the child's connections by means of the placenta with its mother, and through her with the external world.

The Phylogenetic Influence.-This influence may be regarded as the controlling power which initiates the development of the fertilised germinal cell, and shapes its course through embryonic life, until finally it assumes the form of a human child. Though we do not understand the nature of this influence, we may conclude that in the majority of instances it acts along the same lines and towards the same goal-namely, the formation of a healthy foetus. At any rate, we know that in a large number of cases in which a child is congenitally deformed, we are able to trace the cause to conditions which arose during the progress of development: that is to say, the deformity is not due to any phylogenetic or hereditary factor. Of these we shall give examples later. On the other hand, we have equally sure ground for the belief that a hereditary influence may in some cases be itself abnormal. This is proved by instances in which some anatomical or physiological defect or peculiarity is transmitted through succeeding generations of a family. Thus certain defects of sight, such as night-blindness, have been traced through ten generations. Many instances are on record of a family inheritance of structural defects, such as webbed fingers or toes, while among the inherited physiological peculiarities we find such conditions as mericismus (chewing the cud), diabetes insipidus, hæmophilia, and alcaptonuria. 
The point of practical interest is that though the phylogeny of a child is all-important in development, its nature is at present so little understood that we can take few if any active measures to direct its action. This passive attitude on our part is not, however, likely to continue indefinitely. When the science of eugenics has thrown light on the factors involved in the production of normal and of abnormal children, we shall be able to see in what directions we can modify these factors for the good of the child.

The Influence of the Intra-uterine Environment.-In order to appreciate the influence exerted by the intra-uterine environment, we must picture the immediate physical surroundings of the developing child. While still embryonic, it becomes enclosed in the amnion, which forms an enveloping membranous sac attached to the umbilicus and filled with amniotic fluid. Outside the amnion lie the thick walls of the uterus, which is itself covered by the abdominal walls of the mother. This triple casing serves as a most effectual protection against almost all kinds of extraneous injury, while the fact that the child lies actually suspended in a liquid medium safeguards it against any transmitted mechanical shocks.

One force, however, can still find its way to the embryo -namely, the force of gravity. To what extent and in what direction the development is influenced by gravity cannot be stated, but the force is undoubtedly not without its effect. We have no facilities for carrying out experimental observations with respect to the human embryo, but experiments on animals have proved how profound are the alterations in structure produced by varying conditions of gravity. Thus it has been shown by numerous observers that by standing an incubating egg with its long axis vertical, we can produce a deformity of the chick. Similarly, alterations in the position of the ovum in amphibian eggs may lead to malformation. We should mention here that striking deformities may be produced experimentally in animals by very simple means. If an egg is varnished over part of its shell, so as to prevent access of air to the interior, a chick with two heads may be produced. By adding sodium bromide and sodium chloride to the water in which the eggs of the common 
toad (Bufo vulgaris) are floating, headless embryos are formed.

Returning to the human embryo, we find that the developing child, in spite of its isolation from the outside world, is exposed to numerous intra-uterine conditions which are capable of seriously affecting its healthy development. If these conditions are in operation during the earliest months of pregnancy, before the embryo has attained its human form, congenital deformities are likely to occur. In our present knowledge we cannot assert that all varieties of congenital deformity are thus induced, but with many varieties we have ample proof of this relation. Thus it sometimes happens that adhesions-perhaps the result of inflammation-form between the amnion and the embryo. The resulting tension may produce serious deformity by preventing the union of the body-walls in the middle line, and may even drag on such viscera as the heart, the brain, or the intestines, until they come to protrude outside the body. In other cases, when the amnion adheres to the posterior end of the embryo, pressure is exerted on the developing legs, with the result that the two limbs are completely fused together and the so-called "siren" monster is produced. In yet other cases, bands formed in connection with the amnion get tightly twisted round the arm or the leg of a foetus, and the limb becomes amputated; at birth the severed part may be found loose in the amniotic cavity.

In each of the foregoing examples, the cause of the deformity, though lying within the uterus, is external to the embryo itself. Sometimes, however, the cause bears an even more intimate relation to the child. The production of certain malformations has been traced to disease arising in the embryo while its organs are still in progress of formation. Perhaps the best instance of this is provided by dropsy of the cerebro-spinal canal. This pathological condition plays an important part in originating abnormalities of the brain and of the spinal cord. To it has been ascribed the condition (anencephalus) in which a child at birth is found to have no brain and no vault to its cranium-a deformity attributed to pressure of the dropsical fluid which ruptures the head at an early stage of its development. The 
interesting deformity of a child born without a proper heart is also intimately related to the internal physiology of the embryo. These instances of "acardiacus" occur only in twin conceptions, and are produced when one twin succeeds in monopolising the placenta to the exclusion of its fellow. The allantois of the latter becomes attached, not to the maternal tissue, but to the placenta, or even to the umbilical cord of the more fortunate embryo. The circulations of the twins are thus placed in direct communication. Only one heart is needed to maintain the double flow of blood, and this work is gradually assumed by the more vigorous embryo. As a consequence, the heart of the second embryo is deprived of its function, and ceases to develop.

The Influence of the Placenta.-By the end of the third month of fotal life, the embryo has established a new and important connection with its surroundings. With the development of the placenta its physiology undergoes considerable modification, and it is now brought into a more intimate relation with its mother. The placenta establishes a close connection with the maternal circulation, and acts as the foetal organ of nutrition, of respiration, and of excretion. The future development of the child depends essentially on the degree and on the nature of the exchange that takes place through the placenta. So long as the child can obtain the nourishment and oxygen necessary for its growth, and can transfer to the maternal blood-stream the waste products of its own activity, its healthy development is likely to continue without interruption. But if for any reason the placenta fails in this purpose, or allows the passage of harmful material from the mother's blood, the health of the child is likely to suffer. The ill-effects do not now show themselves as deformities, because the embryonic stage-the period when the different organs were in process of formation-has already been completed. They take the form rather, of malnutrition, of fotal disease, or even of death.

The prominent causes of these ill-effects are those morbid states of health of the mother, which affect the composition of her blood. Though these pathogenic influences may be transmitted to her child through the medium of the liquor amnii, they usually pass by way of the placenta. Probably the 
most important of the diseases which are thus conveyed is syphilis-a disease that is responsible for large numbers of deaths, both in utero and after birth. Chronic poisonings, especially by lead and mercury, are well-recognised maternal diseases which lead to premature death of the foetal child. Certain of the specific fevers are known to affect the child in utero. There is reason to suppose that fotal rickets (achondroplasia) depends on maternal causes. Any of these conditions, which may lead to the death of the fœtus either before or after it has left the uterus, may, in some cases, stop short of a fatal issue, and yet may seriously interfere with the nutrition of the child. This result cannot fail to exercise a deep influence on its welfare after birth.

In mentioning the specific fevers, we have raised the interesting and important question as to whether the placenta is able to protect the child to any material extent against infection by the mother. The intimate connection that exists in the placenta between the circulations of the fotal child and of the mother, exposes the former to infection by many of the bacterial and protozoal diseases. Thus small-pox, malaria, anthrax, yellow fever, erysipelas, typhoid fever, and cholera are some of the diseases thus transmitted. Not only may bacterial toxins pass across the placenta, but we have proof that the bacteria themselves may gain access to the child through the same channel. In these cases the infection is necessarily by the blood-stream, and therefore the resulting lesions in the child are likely to be different from those in the mother, in whom the infection was very probably local.

The foregoing paragraphs clearly show us that the health of the child is intimately dependent on the health of the mother. Since, therefore, definite disease in the mother can produce definite disease in the child, we can only conclude that her slighter affections will not be without their similar effect. In other words, that the protection of the mother's health is essential to the future health of the child.

Another matter that requires brief mention is the possibly injurious effect of maternal alcoholism. We know that alcohol can pass across the placenta from mother to child, but with 
what result to the latter we cannot at present state. We must be cautious, therefore, in attributing postnatal defects to this particular antenatal cause. The relation is, unfortunately, not easy to prove. Though we must admit that, compared with the offspring of temperate parents, the infants of alcoholic mothers die in greater numbers, this fact may well mean no more than that the drunken mother neglects her child. Stronger evidence would be furnished if these infants could be taken at birth from their evil environment and placed among healthy surroundings. If, with this help, they still died in excessive numbers, the harmful effect of maternal alcoholism would be sufficiently proved.

With regard to the possible influence of the father's health, it is clear that his child cannot be directly infected from this source, unless disease exists in the father at the time of fertilisation. It has been stated that a father suffering at this time from small-pox may infect the child without infecting the mother. Interesting as this observation is from a scientific standpoint, the occurrence must be so excessively rare as to possess little or no practical significance. The question of the transmission of syphilis by means of the spermatozoa is, however, an important matter, but unfortunately this possibility can at present neither be asserted nor denied. The influence of paternal lead-poisoning is almost equally uncertain, though recently, evidence has been brought forward in France, which suggests that the disease in the father may lead to miscarriages in the mother, or to an excessive proportion of still-births.

Before concluding the question of the placental influence, we must briefly mention two additional factors that might affect the child through this channel. The opinion is held by many that insufficient nourishment of the mother during gestation is likely to retard the development of the child. There is a limited amount of evidence that mothers who are well fed during the concluding months of pregnancy produce somewhat larger children than those who undergo privation. However, there are cases which point in the opposite direction. They are common enough among the poor, where mothers who have been insufficiently fed throughout the whole period of gestation, nevertheless produce strong and well-developed babies. Further, 
it has yet to be shown that the weight of a child at birth is an exact indication of its healthiness. The second factor is the influence of physical work during the later months of pregnancy. It is commonly held that if women are employed in arduous occupations up to the time of their confinement, their infants are likely to be adversely affected. Here, again, we are without any conclusive proof. Most women engaged in household duties continue them up to the time of their confinement, while in primitive nations it is customary for the women to work in the fields until the last.

In the preceding pages we have a series of examples which shows that causes, comparatively simple in their nature, may produce profound structural - and therefore functionalabnormalities in the fotal child. Though in each of the examples the result is of a striking character, we cannot doubt that there must be many less serious influences which will produce milder results. In other words, the embryo and the foetal child are continually exposed to influences that tend to modify their development. Only when these influences are present to an average degree and operate in an average way will an average or normal child be the result. On occasions when they happen to act in a particularly favourable way, the development of the child or of certain of its organs will be more than usually favoured. Under converse conditions the development of the child or of certain of its organs will be adversely influenced. The degree of development of one organ and its function will react on other organs and their functions, and these in their turn will modify others. When, further, we remember that an individual is an expression of his physiological processes, and that an alteration in one single function may profoundly change his whole being, we can understand the essential importance of the intra-uterine environment, not only on the physical, but also on the mental welfare of the future child and man.

Vitally important as is this conclusion, we find at the present day but little disposition to apply it in practice. This indifference is accounted for, perhaps, by the imperfection of our knowledge of intra-uterine conditions of life. We do not yet 
know enough of their nature, of their modes of action and interaction, or of the nature of the reaction of the embryo, to enable us to employ with any certainty the measures that will ensure the safe development of the child. We can neither counteract the influences that are harmful, nor encourage those that are beneficial. We can recognise them only by their effects, and even then not until long after possible harm has been done. We can, however, appreciate the necessity of some preventive measures. We know that the temporary impairments of the mother's health are likely to be the permanent injuries to the child's health. We cannot, therefore, shut our eyes to the necessity and the wisdom of affording a woman, during her pregnancy, the advantages of modern medical science, so that by protecting her from disease we may ensure the health of a future generation. It is important for us to remember that to a probably considerable extent the general outline of a child's future course has already been settled by the time of birth.

The Physiology of the Fatal Child at Term.-Having now briefly traced the principal conditions which influence development in utero, we must complete the subject by a short account of the physiology of a healthy child near the end of its fotal life.

So long as its mother remains healthy, the child is completely shielded from the innumerable factors in the external world which, later, will tend to upset its physiological equilibrium. These factors, which in the adult are usually dependent on vegetable or animal parasites, on abnormal nourishment, or on excessive or deficient use of healthy functions, cannot, if the mother remains healthy, affect the child. It lives, therefore, in sterile surroundings, absolved from any necessity to resist bacterial or other parasitic influences. Its supply of nourishment through the placenta is sufficiently constant in quality and in quantity to maintain it in health. Excessive or deficient use of any of its functions cannot arise in a healthy environment except in response to voluntary effort-a contingency which is of course impossible in fotal life. Nature therefore provides every opportunity for the favourable development of the fœtal child. 
If we enquire into the physiological condition of individual organs, we find that experimental evidence is almost completely lacking. Certain facts, however, can be stated. We might perhaps be inclined to suppose that because the fotal and the maternal circulations are physiologically continuous, and the maternal viscera are all in active function, therefore no functional demand would be placed on most of the fœtal organs, and that they would remain dormant until birth. Without doubt, certain of these do remain thus functionless. In other cases the reverse is true. Of many, however, we cannot speak definitely.

Among the first group, the most important are the lungs, whose aërating function is wholly performed through the placenta by the corresponding maternal organs. It is not surprising, therefore, to find that a child may continue to live up to the time of its birth, even though its lungs are extensively diseased. In addition to aërating the blood, the lungs, after birth, possess a second important function, though this likewise is undeveloped in fotal life. By virtue of their elasticity, they produce a negative pressure in the thorax which very materially aids the heart in maintaining the circulation of the blood. In the absence of this mechanical help, additional work is necessarily thrown on the fotal ventricles, whose total musculature must therefore possess sufficient power to carry on the circulation unaided. Further consideration of this point will be postponed until we discuss the effects of the assumption of the pulmonary functions after birth. Among the other structures which are as yet functionless are the organs of digestion. The alimentary canal has not assumed the power either of digestion or of absorption. The former process is carried on exclusively by the mother; the latter is undertaken by the placenta. The cerebrum is another viscus whose special functions do not develop until later.

Of those organs which are undoubtedly active before birth, we may mention the heart, the liver, the spinal cord, and the medulla. The rhythmical contraction of the foetal heart is well known. Analysis of the foetal liver has proved that the hepatic cells possess the glycogenic function; and the excretion of bile 
into the intestine, where it collects to form meconium, establishes their ability to break down hamoglobin. The reflex action of the cord is shown by such muscular movements of the child as kicking and by the reflex grasping of, for example, an examining finger introduced into the uterus. Deglutition, which is a reflex action dependent on the medulla, may occur in foetal life, as is shown by the presence in the stomach of amniotic fluid which has been swallowed by the child. Some organs, such as the kidneys, may function to a slight extent while leaving the bulk of their work to the mother. Commonly, urine is present in the bladders of children at birth, yet, on the other hand, advanced cystic degeneration of the kidneys of the child is compatible with fotal life. To what degree the ductless glands are active before birth cannot be definitely stated, owing to our present ignorance of their functions. The thymus gland and the adrenal glands are relatively large in fotal life-a fact which probably indicates their physiological importance at that time. On the other hand, the adrenals are said not to contain their active principle, adrenalin, until some time after birth. The thyroid gland is known to secrete colloid before the second month of fœtal life, but the secretion has been found to possess chemical differences from the colloid produced after birth.

In general terms we may say that the activity or inactivity of any fotal viscus is determined by the demand on its function. Once the viscus has become histologically specialised, it seems to possess the power of active functioning, and it remains quiescent only so long as its work is performed by the corresponding maternal organ. For example, instances have been recorded of goitrous infants born to mothers with atrophied thyroid glands. In these cases the fœtal organ has not only served the requirements of the child, but has also supplemented the deficiency of the mother. Removal of the thyroid gland from pregnant dogs has produced enlarged thyroids in their puppies. With regard to the earliest date in fotal life at which the different functions can become active, we cannot speak definitely. Up to the sixth or seventh month many of them are in abeyance. This fact, of course, does not imply that they cannot, if required, become active, because in children who are 
born prematurely, all their functions can come into healthy action even at this early period.

\section{II.-NATAL Physiology}

It has been the custom too often to look on the child before birth and the child after birth as organisms widely separated from each other. Medical interest in childhood has, to a large extent, stopped short at the new-born infant, and has left the problems of intra-uterine physiology and pathology to biological investigators. This is to be regretted. The fotal child and the new-born child are one and the same organism. There are, however, essential differences in their environments, and we need to ascertain the physiological effects induced thereby. The transition from one environment to the other is effected more or less rapidly, and during its progress the child is temporarily exposed to certain new conditions. As soon as the change has been completed, the child is required to make rapid readjustments in its economy, in order to save its life. The important physiological changes that take place within these few hours or days must now be considered.

We recognise two distinct influences that are exerted at this period. First, the mechanical forces and resistances which bring about the expulsion of the child from the uterus. Next, the highly complex influence that comes into action at the moment when the child is deprived of its placental functions, and is compelled to establish new channels for obtaining its nourishment and its oxygen, and for disposing of its excretion.

The outstanding features of the mechanical forces of parturition are their temporary action and their great intensity. It is the former characteristic which enables the child to be subjected to them and yet to survive. When, however, labour is prolonged, owing, for example, to inadequate diameters of the maternal pelvis, the death of the infant may occur from the sustained pressure alone, without any obvious injury. In these cases the fatal result is usually attributable to the severe compression of the brain. On all occasions very material assistance is conferred on the child by virtue of the softness and the 
flexibility of its tissues by which it is able to accommodate itself to surrounding pressures. Its maximum diameters being those of the head and the thorax, these parts are exposed to the severest strain, and the results are often serious and permanent. Of these we may mention meningeal and cerebral hæmorrhages. Each has a deep effect on the subsequent history of the child, and often leads to paralysis, backward mental development, and even idiocy. In such cases the degree of the mental deficiency varies with the extent of the injury. Pressure on the thorax is sustained by the shoulder-girdle, and is not generally harmful. This is in remarkable contrast to the fatal effect of pressure on the thorax of a child at any time after birth, the difference being due, of course, to the presence or absence of pulmonary respiration.

The physiological changes occurring during the few days immediately succeeding birth are more radical and more manifold than at any other period. They are, essentially, attempts on the part of the organism to accommodate itself to new conditions. Throughout fotal life the most important organ is the placenta. By the end of parturition this organ is totally destroyed physiologically. Its functions, however, are still essential to the child, who is therefore compelled to find other means of carrying them on without interruption. The most urgent demand is for oxygen, and therefore the first effort of the new-born infant is directed to secure a fresh supply. The nervous mechanism of the first respiration is commonly stated to be a reflex due to stimulation of the skin by the cold air. This explanation is not convincing, and does not harmonise with many established facts. The first breath is often postponed until long after the time required for the completion of the reflex arc. A child presenting by the breech will often make respiratory efforts while the greater part of its skin is still protected from the air. It is not recorded that infants born in climates where the atmospheric temperature is as great or greater than that of the maternal tissues are defective in their first breaths. Again, new-born animals begin to breathe, even when their skins are protected from the air by a covering of fur. 
The first respiration is due to stimulation of the respiratory centre in the medulla by blood which is surcharged with carbon dioxide and deficient in oxygen. This condition arises only when the placental circulation fails-as a rule, not until the child has been born-but in breech presentation it may result earlier. Premature efforts of respiration under these circumstances are recognised by obstetricians as evidence that the child's head has compressed the umbilical cord against the maternal pelvis, and that the placental circulation has been stopped. Death by suffocation will follow unless this pressure is speedily released.

As soon as the child has taken his first breath, a far-reaching sequence of events is produced, which does not end until fundamental changes have been effected in the heart. These changes are as follows:-Throughout fotal life the thorax has accommodated the heart, the thymus gland, and the lungs-the last named, however, only in a collapsed or solid condition. Its capacity is considerably increased by the first muscular effort of inspiration. The negative intrathoracic pressure thus produced compels the lungs to expand, sucks open their alveoli, and fills them with air, which rushes in along the trachea and the bronchi. The lungs themselves, being elastic, resist this expansion. So soon as the inspiratory movement ceases, they contract again, the chest-ivalls subside, and the first expiration has taken place. The lungs, however, do not collapse to their original fotal volume, and therefore the chest remains at a somewhat greater capacity than before, the exact position that it assumes representing an equilibrium between the elastic pull of the lungs and the resistance of the ribs. As results of the first complete respiration, whereby the diameters of the chest are increased, two important changes are brought about: (I) a negative pressure is established in the chest, and (2) the blood-capillaries in the lungs are opened up and a greatly increased quantity of blood passes through them. Each of these changes produces secondary effects, which are themselves of great importance.

(I) The negative pressure that is established in the chest becomes, as is well known, an important factor in the mainten- 
ance of the circulation. By its help the blood that has been pumped by the heart into the arteries, and so to the capillaries, is aspirated from the veins into the right auricle. Further, the descent of the diaphragm with each inspiration increases the intra-abdominal pressure, and therefore squeezes blood along the inferior vena cava towards the heart. Both these mechanical aids are necessarily absent in the fotus. We can readily understand how the amplitude of the respiratory movements and the increase in the capacity of the thorax at birth must directly influence the vigour of the circulation, and therefore the vitality of the child. Infants whose respiratory mechanism is so insufficient to expand their lungs as to cause congenital atelectasis, almost invariably take shallow breaths, and their cry is feeble. The nutrition of their tissues is imperfect for want of proper aëration of their blood, so that the action of the heart, which is already over-taxed by the absence of its respiratory pump, is further weakened. These infants are liable to die within a short time of birth.

(2) The lungs, which have hitherto required only sufficient blood to supply the nutritive demands of their tissue, are now called on to aërate the whole of the blood. An urgent necessity therefore arises for a great increase in the pulmonary circulation. To this end the pulmonary capillaries are opened up to give passage to a greater volume of blood. In order to maintain this extra flow, the small pulmonary arteries (right and left) must have their sectional areas correspondingly enlarged. As soon as this alteration is effected, a much greater proportion of the blood, pumped into the pulmonary artery by the right ventricle, begins to pass into its right and left branches, and therefore less flows along the ductus arteriosus. This vessel, deprived of its function, begins to decrease in diameter, until within a few days its lumen is completely occluded.

Another effect of the alteration in the pulmonary circulation is seen in the closure of the foramen ovale between the two auricles. Previous to birth, except for a small quantity of blood returned by the pulmonary veins from the inactive lungs, the sole supply of blood to the left auricle has been through this 
opening. But as soon as respiration starts, the left auricle is filled by the increased flow along the pulmonary veins, the passage of blood from the right auricle through the foramen ovale is hindered, and this opening, no longer required, gradually closes.

As a result of these changes, the amount of work performed by the two ventricles of the heart-which at birth have walls of equal thickness-undergoes alteration. The left ventricle, which has hitherto been concerned in maintaining the circulation through the head and the upper extremities, now becomes responsible for the circulation to the whole body. The sum total of its work is increased, in spite of the fact that it is helped by the negative thoracic pressure and by the vigorous muscular movements of the infant. To cope with these altered conditions, its walls begin to hypertrophy. The right ventricle, on the other hand, has, during fotal life, been engaged principally in carrying on the circulation through the vessels of the trunk, of the lower extremities, and of the placenta. After birth its range of action is restricted to the lesser circulation through the lungs, and its work is considerably curtailed. Its muscle-walls are no longer required to exert the same power as before, and a marked difference in thickness, which remains throughout life, thus arises between the two ventricular walls. ${ }^{1}$

Summarising the changes in the heart consequent on birth, we find that they are the direct result of the respiratory movements which, partly by increasing the flow of blood through the lungs and partly by establishing a negative thoracic pressure, fundamentally modify the conditions of the circulation. The aërating function is transferred from the systemic to the pulmonary circulation, and with the full appearance of this double system, extra work is thrown on the left side of the heart. The left ventricle being now exclusively responsible for the whole of the systemic circulation, hypertrophies in excess of the right ventricle, whose work is lightened to the extent that

1 An intimate relation exists between the causation of the so-called "congenital heart-disease" and the conditions of the lungs (and therefore of the heart) before and after birth. This question hardly falls within the scope of the present chapter, and its discussion must be deferred. 
it now maintains the flow through the lungs alone instead of through the trunk, the legs, and the placenta. The negative thoracic pressure, the increased abdominal pressure caused by the descent of the diaphragm, and the muscular movements of the infant, now for the first time assist the action of the heart. 


\section{CHAPTER II}

\section{THE PHYSIOLOGY OF CHILDREN (concluded)}

Postnatal Physiology. Physiology of Growth-Interdependence of Organs; Changes in the Skeleton and in Bodily Proportions; Growth in Height; Growth in Weight; Growth of Individual Organs. Physiology of Certain SPecial Functions-Digestion; Circulation and Respiration; Body-weight and Surface-area; Kidneys; Sweat; Carbon Dioxide; Respiratory Exchange; Lymphoid Tissue. Physiology OF METABOLISM-Experimental Methods; Growth and Animal Heat ; Heat-Regulation ; Its Development ; Nutrition ; Caloric Requirements of an Infant ; Caloric Values of Food-stuffs ; Respiratory Quotient ; Growth and Heat-Production; Age not the Criterion of Food Requirements; Surface-area per kilo. Body-weight; Principles of Infant Feeding; Total Heat-loss ; Total Minimum of Energy required ; Human Milk; Its Calorific Value; The Quantity Required; Cow's Milk ; Its Calorific Value ; The Quantity Required ; Influence of Musclework; Of Atmospheric Temperature; Size and Frequency of Feeds ; The Feeding of Older Children. PUBERTy.

\section{III.-Postnatal Physiology}

As soon as the new-born infant has completed the immediate adjustments demanded by an altered environment, his physiology closely approximates to that of an adult. The numerous resemblances need not be touched on in this chapter. The differences, however-for the most part only matters of detailare sufficient to confer important characteristics on his physiology. These points we shall now consider. The most fundamental distinction is the phenomenon of growth, and this subject will receive our first attention. We shall then deal with the functions of individual organs-excluding those of the nervous system, which will be reserved for the two next chapters-and then pass to the question of metabolism. In the light of the 
knowledge thus gathered, we shall discuss the important subject of nutrition. In conclusion, we shall briefly refer to the physical changes associated with the advent of puberty: This chapter, therefore, will be divided into four sections :-

I. The Physiology of Growth ;

2. The Physiology of certain Special Functions;

3. The Physiology of Metabolism, including Animal Heat and Nutrition;

4. The Physiology of Puberty.

\section{The Physiology of Growth}

An infant is not merely a small replica, anatomical and physiological, of an adult. The process of growth consists of something more than an addition to his weight, volume, and height. Not only does it involve, as we shall see later, changes in the chemical composition of his body, but it also implies alterations in the relative sizes and weights of every viscus. These alterations, carried on persistently but gradually over many years, prove that his several physiological functions are undergoing quantitative if not qualitative changes. Some functions rise in importance, others decline; and since the organism does not maintain superfluous tissues, the less necessary a function becomes, the smaller does the particular organ grow. Month by month, even day by day, the child's physiology passes through some modification. Every such change affects the general physiology directly, but it also starts a whole series of secondary changes, which produce their influence even on remote parts of the body.

Let us consider, by way of example, the changes in the lower jaw. At birth the jaw is relatively undeveloped, and consists of a mere shell of bone. Soon it is called on to accommodate the milk-teeth, and later, the permanent teeth. Simultaneously it is required to withstand the strain of the powerful muscles of mastication. In response to these demands, it alters its shape, and becomes a larger and more prominent feature of the face, and its weight increases considerably. Numerous secondary results must follow. The temporal bones, 
to which the jaw is slung, must become stronger and heavier. The weight of the skull, transmitted down the spine, is increased; the vertebræ must themselves become stronger, and the effect will be felt by the sacrum, the pelvic bones, and the legs. The ligaments connecting these bones will be thickened. Simultaneously the muscles that nod and turn the head will be called on to deal with a heavier jaw, and they must become more powerful. The bony points from which they arise must grow larger, in order to resist the increased force of the contractions. Again, the jaw and all these other bones will require a greater supply of nourishment. Their blood-capillaries and their arterioles must therefore increase in number, and the effect of this will be felt, not only in the inferior dental and the internal maxillary arteries, but in the external carotid, and so back to the heart, which must itself arrange to cope with the increase in its peripheral circulation. Further, the lungs must be prepared to excrete an increased quantity of carbon dioxide.

This illustration is in part anatomical, but we cannot doubt that functional changes in any one viscus must affect the functional activities of many if not all the other viscera. A child, beginning to walk and to run, makes frequent use of muscular contraction, and produces an augmented volume of carbon dioxide. Extra work is therefore thrown on the lungs, and the pulmonary circulation must be accelerated. This in its turn implies an increased activity of the heart, and therefore the systemic circulation is quickened. Each organ now receives the stimulus of extra nourishment, and cannot fail to have its activity and its growth modified.

These examples are sufficient to indicate how intimately the organs of the body depend on a change in any one of them. Without further attempting the difficult task of unravelling their manifold correlations, we may approach the phenomena of growth from four standpoints. First, we may compare the skeleton and the, bodily proportions of the infant at birth with those of the fully-grown man. Next, we may study growth as revealed in increase of height. We may observe it again in changes of weight. Finally, we may consider the individual growth of special organs. 
Changes in the Skeleton and in Bodily Proportion.-The essential function of the skeleton being to provide a supporting framework to the softer and more yielding tissues, we find that the size and the shape of the bones are specially influenced by mechanical forces. Of these, the first in importance, because it is always in action, is gravity. A second force is muscular contraction. Its influence, as affecting any particular bone, varies much from time to time, but its effect is nevertheless great in producing alterations in the strength and form of a bone. The ligaments also exercise considerable strain. Finally, the pressure set up by relatively soft but growing organs, such as the heart and lungs, or by the development of structures intended to subserve a physiological function, such as the teeth, may directly mould the bones in the immediate neighbourhood.

When we examine the skeleton of an infant for the results of these mechanical forces, we find that the bones show but slight effects compared with those that will be impressed on them in later years. They have been subjected to these forces for too short a time, and only under special conditions. Gravity has, of course, made itself felt in utero, but as the fœtus is equally at home in all positions, whether horizontal or vertical, its bones have not been called on to transmit weight in one direction more than in another. The effect of gravity, therefore, has not been constant before birth, as it becomes later, especially when an upright attitude is adopted. Muscular movements in utero have been neither free nor forcible, and owing to the frequent changes in the position of the foetus, a muscle that contracts on one occasion against gravity may at its next contraction be assisted by gravity. The effect on the bones is therefore slight. For similar reasons the ligaments do not produce much change. With regard to the growth of soft organs, these play their part even before birth (witness the effect of the brain on the skull, or the liver on the lower ribs), and such changes are continued throughout all the years of extra-uterine life.

Bearing in mind these mechanical differences between the life of a child before and after birth, we shall be able to understand the many distinctions between the infantile and the adult 
skeletons. On examining the former, ${ }^{1}$ the peculiarity first noticed is in the relative proportions of the body-the head, the trunk, and the legs. The head is remarkably large, and is no less than one-fourth of the infant's total length, whereas in the adult the proportion is only one-eighth. The legs, on the contrary, are relatively short, measuring only about threeeighths of the height, as compared with four-eighths in the adult. The ratio, therefore, of head to leg, which in the adult is I to 4 , is, in the infant, 2 to 3 . The outstanding impression obtained by a cursory glance at the skeleton, is that the body is too big for the legs and the head too big for the body. ${ }^{2}$

In connection with this relative shortness of the legs, the mid-point of the body-obtained by bisecting the height-lies some 2 to $3 \mathrm{~cm}$. above the navel in infancy. Together with the centre of gravity, it descends with increasing age, because the legs grow faster than either the trunk or the head. At eighteen months it corresponds to the navel, while at ten years it is $2 \mathrm{I} \mathrm{cm}$. below. In the full-grown man it has sunk to the level of the pubes. Coincident with this change, the sitting height, as compared with the standing height, becomes less.

Passing now from these general features to a more detailed examination of the skeleton, we find that the head itself is remarkable for the large size of the cranium and the small size of the face. This is due partly to the volume of the infant's brain, which is relatively larger at birth than at any subsequent age, and partly to the undeveloped condition of the face. The lower jaw, with its milk-teeth and its muscles of mastication still immature, is particularly small. The distance from the mouth to the point of the receding chin is therefore short. Similarly the upper jaw is but little developed, because its alveolar processes do not attain their full proportions until the upper teeth have been cut, and the antrum of Highmore, though present, is small. The vertical dimension of the bone is therefore short and the mouth is separated from

1 The account that follows is based on a comparison of the series of skeletons of new-born children in the Hunterian Museum of the Royal College of Surgeons with that of an adult.

2 Sec Frontispiece. 
the orbital cavities only by a narrow interval. Since the face is largely composed of the upper and lower jaws, it is, in the infant, overshadowed by the cranium. The effect is enhanced by the undeveloped state of the frontal sinuses and the superciliary ridges, so that the frontal bone sweeps down in an uninterrupted curve from the crown of the head to the level of the eyes.

The next series of changes to be noted are for the most part expressions of the effect of gravity. From the time when the infant begins to walk, his weight is transmitted along the vertebral column to the pelvic bones, and thence through the legs to the ground. This force, acting for hours daily, speedily modifies all these structures, and they lose their characteristic infantile form. At birth the vertebral column runs an almost straight course from the base of the skull to the pelvis, with only a suggestion of the dorsal curve, and none at all of the cervical and lumbar curves. The sacro-vertebral prominence is but slightly marked. The sacrum is concave from side to side, but is relatively flat from above downwards. The posterior iliac spines project only a little distance behind it, and its anterior surface looks forward rather than forward and downward. The pelvis is very small even in proportion to the size of the child, and its transverse diameter is decreased. The iliac fossæ are spread out, so that they look more forward, and they are flat rather than curved, the anterior ends of their crests not being bent inward as in later life. The obturator foramen is more circular in outline, the subpubic angle more acute, and the tubera ischii closer together. In the lower extremities, we notice that the femora run more vertically, and their shafts are but little curved. The os calcis is less prominent, and the arch of the foot is not well developed.

All these points undergo gradual alteration, especially from the time when the child begins to assume the erect posture, and gravity begins to act parallel to the length of the body. In order to increase its mobility and to minimise shocks transmitted from the feet to the head-as in jumping-the vertebral column assumes characteristic curves in the cervical, dorsal, and lumbar regions. The sacrum, now called on to support the 
weight of the head, the trunk, and the arms, becomes more deeply wedged into the pelvic girdle, so that the posterior iliac spines are left projecting behind. At the same time, it is flattened from side to side by the weight of the body. The sacral promontory is rotated forward-a change which, with the development of the lumbar curve, makes the sacro-vertebral angle prominent-but as the lower end of the bone is held in position by the sacro-sciatic ligaments, the anterior surface of the sacrum becomes markedly concave in vertical section and its upper part looks more down than before. These alterations, by diminishing the antero-posterior diameter of the pelvis, and accentuating the curve of the lateral pelvic walls, tend to increase the transverse diameter relatively.

In addition to the foregoing alterations, gravity produces certain other changes in the pelvis when the body-weight is transmitted to the ground or other support directly from the pelvic bones, as in the position of sitting. The resistance of the ground, acting upwards, widens the interval between the ischial tuberosities and opens up the subpubic angle.

The iliac fossæe change in shape as the result of the tension of the muscles to which they give attachment. The pull they exert causes the fossæ to become more curved and brings the anterior superior spines nearer to the middle line.

As the transverse diameter of the pelvis becomes greater, the upper extremities of the femora are moved farther apart, and the oblique direction of their shafts is necessarily increased. A second change in the femur is seen in the curve which runs the whole length of the bone. In the foot the important change is the development of the plantar arch. Its purpose is similar to that of the curves in the vertebral column. Another change in the foot, the result of the powerful action of the calf-muscles, both in walking and in standing, is the great enlargement of the os calcis, so that it projects prominently at the heel.

The arm-bones of the new-born infant call only for short notice, as their characteristics are not very unlike those of the adult $-a$ fact that must be connected with their freedom from supporting any of the body-weight. They are, however, 
relatively shorter, but as soon as the child masters their use, they begin to lengthen, and within a few years they bear practically the same proportion to the height as in adults. The clavicle is short, and therefore helps to produce the narrow, sloping shoulders of the infant. The breadth of the scapula, compared with its length, is greater, and the subscapular fossa is only slightly hollowed. The ulna and the radius are more nearly equal in thickness than in the adult.

Apart from the mechanical influence of gravity and of muscular or ligamentous strain, another force exercises a profound effect on the new-born infant's skeleton. This is atmospheric pressure, and its effects are to be found in the bony walls of the thorax. The shape of the thorax in early infancy is very markedly different from that of an adult or even of a child. We have already had occasion in the previous chapter to speak of the negative pressure that is established in the thorax as soon as respiration begins. This internal negative pressure, aided by the positive atmospheric pressure without, produces marked changes in the ribs and sternum. These changes are at once apparent when we compare the infantile with the adult thorax. The features of the former are as follow:-Its outline is relatively simple. With its minimum circumference above, its diameters increase more or less uniformly from above downwards. Its shape is therefore that of a dome, or, rather, of a funnel. The sternum passes downwards and forwards in a fairly straight course, giving a relative value to the antero-posterior diameter of the chest. The first and, to a smaller degree, the second ribs run only slightly obliquely, and therefore the level of the upper border of the sternum is high compared with the dorsal vertebræ. As a consequence, the plane of the thoracic inlet is inclined only moderately downwards and forwards. The ribs themselves show some striking distinctions. Starting from their spinal attachments, they pass almost directly outward. There is therefore no longitudinal furrow running along each side of the spine, and as a result the infant's back has a relatively flat surface. Continuing their course, the ribs curve to the front only gradually, and they end with their anterior extremities 
pointing forwards instead of looking inwards towards the sternum.

By way of contrast, we may note that in the adult thorax the antero-posterior diameter is shorter, the transverse diameter reaches a maximum some distance above the lower border of the cavity, and the posterior surface of the sternum and the curve of the dorsal spine are concave towards the interior of the chest. The cavity has therefore completely lost its funnel shape, and, viewed from the front, tapers above and below. The plane of its inlet is more oblique. The ribs at first run backwards as well as outwards as far as their angles, thus producing longitudinal furrows down the back, in which the erector muscles of the back are accommodated. They then turn sharply forward, sweeping round the sides of the chest and bending inwards at their anterior ends to face the sternum.

Growth in Height.-Growth in height has two periods of maximum intensity. The first is during the few months after birth, the second begins just before puberty-in girls about the twelfth year, and a little later in boys. Between these two periods it proceeds more gradually. After the second period the rate diminishes again, and, according to Hall, ceases within a few years-in girls at 15 , in boys at 17 . Measurements by other observers, however, go to show that growth may be continued beyond these limits.

The length of an infant at birth averages $50 \mathrm{~cm}$. (20 inches). This increases in each of the four quarters of the first year by $9,8,4$, and $3 \mathrm{~cm}$. respectively, so that at the end of the year the infant measures about $75 \mathrm{~cm}$. In each of the three next years the growth is 10,8 , and $5 \mathrm{~cm}$. respectively, so that a child of 4 measures approximately I metre. From this age until the second period of accelerated growth, the annual increase is $5 \mathrm{~cm}$., but by the time puberty begins the figure has risen to 6 or $7 \mathrm{~cm}$. Girls from I I to I4 years outstrip boys, but thereafter boys overtake and pass them.

All these figures are approximate. The following numbers, published in the Report of the Royal Commission on Physical Training (Scotland), show the annual increase in inches for boys and for girls from five years onwards :- 
Groweth in Height of Boys and of Girls from the Fifth to the Seventeenth Year.

\begin{tabular}{|c|c|c|c|c|}
\hline \multirow{2}{*}{ Age. } & \multicolumn{2}{|c|}{ Boys. } & \multicolumn{2}{|c|}{ GirLs. } \\
\hline & $\begin{array}{c}\text { Height } \\
\text { in inches. }\end{array}$ & $\begin{array}{c}\text { Annual } \\
\text { Increase. }\end{array}$ & $\begin{array}{l}\text { Height } \\
\text { in inches. }\end{array}$ & $\begin{array}{l}\text { Annual } \\
\text { Increase. }\end{array}$ \\
\hline 5 & $4 I \cdot 03$ & $2 \cdot 57$ & 40.55 & $2 \cdot 22$ \\
\hline 6 & 44.00 & $2 \cdot 97$ & $42 \cdot 88$ & $2 \cdot 33$ \\
\hline 7 & $45 \cdot 97$ & $\mathrm{I} \cdot 97$ & 44.45 & $\mathrm{r} \cdot 57$ \\
\hline 8 & $47 \cdot 05$ & I.08 & $46 \cdot 60$ & $2 \cdot 15$ \\
\hline 9 & $49 \cdot 70$ & 2.65 & $48 \cdot 73$ & $2 \cdot 13$ \\
\hline 10 & $51 \cdot 84$ & $2 \cdot 14$ & $51 \cdot 05$ & $2 \cdot 32$ \\
\hline I I & 53.50 & I. 66 & $53 \cdot 10$ & 2.05 \\
\hline 12. & 54.99 & I. 49 & 55.66 & $2 \cdot 56$ \\
\hline I 3 & $56.9 \mathrm{I}$ & $I \cdot 92$ & $57 \cdot 77$ & $2 \cdot I I$ \\
\hline I 4 & 59.33 & $2 \cdot 42$ & 59.80 & 2.03 \\
\hline I 5 & $62 \cdot 24$ & $2 \cdot 9 \mathrm{I}$ & 60.93 & $I \cdot I 3$ \\
\hline I6 & $64 \cdot 3 I$ & 2.07 & $6 \mathrm{I} \cdot 75$ & 0.82 \\
\hline I 7 & 66.24 & I.93 & $62 \cdot 52$ & 0.77 \\
\hline
\end{tabular}

Growth in height is subject to certain variations. Thus it shows-except in infants-definite diurnal variations, the morning measurement being from $\mathrm{I}$ to $3 \mathrm{~cm}$. in excess of that in the evening. The difference is the result of the erect posture. During the course of the day, when the weight of the body is transmitted through the vertebral column, each vertebral disc is compressed, the arch of the foot is lowered, and the curve of the spine is increased. Rest in a horizontal position overcomes these changes, and the body gradually returns to its full height. A second factor that influences the increase in height is the season of the year. The rate of growth is fastest from April to July, and least between August and December. Other factors are: race, social conditions, food, and warmth of clothing. Growth is retarded by school-work, but is accelerated by holidays.

Growth in Weight.-Increase in weight follows a curve which rises rapidly at first, but becomes less and less steep until adolescence is completed. Like growth in height, it shows a definite acceleration just before puberty, appearing therefore two years earlier in girls than in boys.

The average weight at birth is 3400 grms. ( $7 \mathrm{lb}$.). It doubles itself in five months, triples itself in another seven, and 
quadruples itself in another eighteen. During the first weeks of life the daily gain is about 30 grms. ( $1 \mathrm{Oz}$.) ; from the third to the fifth weeks, 22 grms. ( $\frac{3}{4} \mathrm{Oz}$.); from the sixth to the eighth weeks, 15 grms. ( $\frac{1}{2} \mathrm{oz}$.); and from then to the end of the year, 12 grms. $\left(\frac{2}{5}\right.$ oz.). The figures representing the average percentage daily increase are as follow:- 1 per cent. in the first month, 0.3 per cent. at the sixth month, and O.I 5 per cent. at I year. As is well known, the first few days of an infant's life are marked by a loss of weight. This fall, which is most pronounced in the first twenty-four hours, is only gradually recovered from, and not until the end of the first week is the original birth weight regained.

The average annual weights of boys and of girls, corresponding to the heights already given, are as follow :-

Growth in Weight of Boys and of Girls from the Fifth to the Seventeenth Year.

\begin{tabular}{|c|c|c|c|c|}
\hline \multirow{2}{*}{ Age. } & \multicolumn{2}{|c|}{ Bors. } & \multicolumn{2}{|c|}{ Girls. } \\
\hline & $\begin{array}{l}\text { Weight } \\
\text { in pounds. }\end{array}$ & $\begin{array}{c}\text { Annual } \\
\text { Increase. }\end{array}$ & $\begin{array}{l}\text { Weight } \\
\text { in pounds. }\end{array}$ & $\begin{array}{c}\text { Annual } \\
\text { Increase. }\end{array}$ \\
\hline 5 & 39.9 & 2.6 & $39 \cdot 2$ & $3 \cdot 1$ \\
\hline 6 & $44 \cdot 4$ & $4 \cdot 5$ & $4 \mathrm{I} \cdot 7$ & $2 \cdot 5$ \\
\hline 7 & $49 \cdot 7$ & $5 \cdot 3$ & $47 \cdot 5$ & 5.8 \\
\hline 8 & $54 \cdot 9$ & $5 \cdot 2$ & $5^{2 \cdot I}$ & $4 \cdot 6$ \\
\hline 9 & 60.4 & $5 \cdot 5$ & $55 \cdot 5$ & $3 \cdot 4$ \\
\hline 10 & $67 \cdot 5$ & $7 \cdot I$ & $62 \cdot 0$ & $6 \cdot 5$ \\
\hline I I & $72 \cdot 0$ & $4 \cdot 5$ & 68.I & $6 \cdot 1$ \\
\hline 12 & $76 \cdot 7$ & $4 \cdot 7$ & $76 \cdot 4$ & $8 \cdot 3$ \\
\hline I 3 & $82 \cdot 6$ & $5 \cdot 9$ & $87 \cdot 2$ & 10.8 \\
\hline I 4 & $92 \cdot 0$ & $9 \cdot 4$ & $96 \cdot 7$ & 9.5 \\
\hline 15 & $102 \cdot 7$ & 10.7 & $106 \cdot 3$ & $9 \cdot 6$ \\
\hline I 6 & I I 9.0 & $16 \cdot 3$ & II $3 \cdot I$ & 6.8 \\
\hline I 7 & 130.9 & II.9 & I I 5.5 & $2 \cdot 4$ \\
\hline
\end{tabular}

Observations on the weight of any given child require to be interpreted with care. So much importance is attached at the present day to increase in weight as a sign of an infant's progress, that it will be advisable to mention the points for which allowance must be made. In the first place, infants do not all grow at the same rate. The percentage increase is a more instructive indication of a child's progress than is the 
absolute increase. Again, an infant's weight is subject to extensive diurnal variations, a difference of as much as 200 grms. $(6 \mathrm{oz}$.) representing the average fall between evening and morning in a child of four months. This loss is readily accounted for. During the day the child takes frequent feeds, and by evening has succeeded in adding to his weight. Throughout the night his feeds are less frequent, and they do not counterbalance the loss of water by the lungs, the skin, and the urine. Other factors which may materially affect the weight are the presence of food in the stomach, of urine in the bladder, or of fæces in the rectum. The difference in weight when these three are full and when they are empty may easily amount to 300 to $400 \mathrm{grms}$. (IO to I4 oz.). Finally, we must recognise that an increase in weight does not necessarily imply an increase in growth. It may merely represent an extra deposit of fat, which may itself be either desirable or the reverse. Similarly, the rapid rise of weight that is often observed in badly-fed children when first they are given suitable food, is not an indication of their growth but of the deposit of fat.

Though these points are of greatest importance in infants, they must not be overlooked in older children. At io years the diurnal variation is given by Camerer as 700 grms. ( $\mathrm{I} \frac{1}{2} \mathrm{lb}$.). Another allowance that must be made for children past the age of infancy, is the seasonal variation which their weights, like their heights, undergo. This variation is the converse of that already mentioned for height, being at a maximum from August to December, but falling to a minimum between April and July.

We may observe from the foregoing sections on height and weight that the connection between the two forms of growth is fairly close. This relation is explained by the fact that growth in weight is largely a question of growth in muscle, and therefore of growth in skeleton, while height, of course, is essentially a skeletal measurement.

Growth of Individual Organs.-The rates of growth of the various viscera are very unequal. All the organs of the body are absolutely heavier in adults than in infants, with two exceptions. These are the adrenal glands and the thymus. The adrenals are particularly large in fotal life. The size of 
each at birth is no less than one-third that of the kidney, and together they constitute I/400th of the body-weight. In the adult they have dwindled to one-fortieth the weight of the kidney, and their absolute weight has slightly decreased, although the absolute weight of the body has increased twenty-fold. The thymus gland is relatively large at birth, and it increases still more during the first two years, but then begins to atrophy. In adult life it is often represented merely by a small undefined mass of fat at the base of the heart.

Though with these two exceptions all the viscera are absolutely heavier in adult years, many of them do not keep pace with the growth of the body as a whole, and their relative weights therefore decline. Among this group are organs of the importance of the heart, the liver, the kidneys, the thyroid gland, and the brain. A few organs, such as the spleen and the stomach, retain their original proportionate weight, while the pancreas, the muscles, and the skeleton become relatively heavier. Of all organs, the one whose relative enlargement is greatest is the testis. This increases to sixty times its absolute weight and to three times its relative weight. The ovary, on the other hand, is relatively a little smaller in adults than in infants.

These facts are summarised in the following table by Vierordt :-

Increase in Weight of Certain Organs from Birth to Adult Age. Body Increase Twenty-fold.

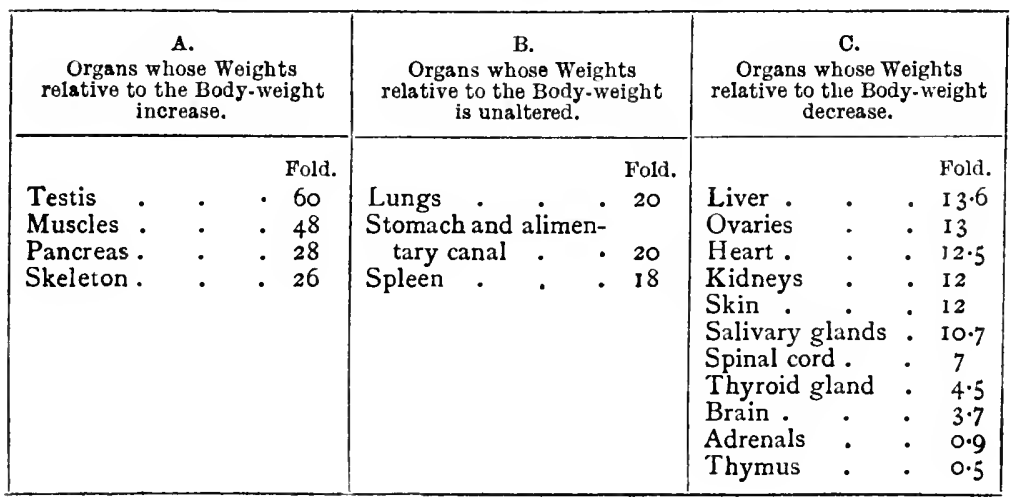


The above figures point to very material physiological alterations during increasing years of childhood, but in our present limited knowledge we cannot do more than speculate as to the causes of these changes in size. Not the least difficult of the problems to be solved arises in connection with the brain, which is three or four times as large, relatively, in infancy as in the full-grown man, despite the fact that its function is completely in abeyance at birth and does not fully develop for many years. Perhaps its considerable growth in intrauterine life is, in part, the result of its specially favourable situation as regards a supply of nourishment. The head and the upper extremities receive a supply of purer blood than the other parts of the body, and their development is to that extent likely to be favoured. In spite, however, of its large size at birth, its essential structural elements-the cortical nerve-cells-are poorly developed. Indeed, Soltmann has shown that the electrical stimulation of the surface of the brain of newborn animals is without effect, but that at a later age it produces violent convulsions.

Although an infant's brain is thus inactive at birth, its rate of growth is very rapid, and by the sixth month its weight has nearly doubled, increasing on an average from 380 grms. to nearly 700 grms. Thereafter its further growth is relatively slow, and by the seventh year the weight is a little over 1300 grms. During these years the rapidity of growth of the different parts of the brain has been unequal, and the frontal lobes-the site of the intellectual centres-do not begin to attain the prominence that is a feature of the adult brain, until the sixth year. The size of the cerebellurn, on the other hand, increases much earlier. Its special function is to maintain equilibrium by co-ordinating the muscles of the body during sitting, standing, walking, and the other movements that require balance. It is a useless organ to the fotal child, and even after birth its function is of but little importance until the child begins to sit up in the latter half of the first year. Accordingly, we find that it is poorly developed at birth, but that by the end of the first year it has grown more rapidly than any other part of the nervous system. In those cases in which its growth is 
defective, equilibration is difficult, and the child suffers from "congenital ataxy."

\section{The Physiology of Certain Special Functions.}

At the outset we must note that the chemical composition of an infant is unlike that of an adult, though the differences are quantitative rather than qualitative. By analysing the frozen and pulverised bodies of still-born infants, it has been shown that they contain less protein and much less inorganic salts, but more fat and more water, than are found in the bodies of adults. The smaller proportions of protein and of inorganic salts are sufficiently accounted for by the slight development of the muscular system, and by the cartilaginous condition of the bones at birth. The fat, which steadily increases in amount throughout fotal life, constitutes 9 per cent. of the newborn infant, as compared with 2.5 per cent. of the adult. The proportion of water, after falling throughout foetal life, stands at birth at 74 per cent., as compared with $6 \mathrm{I}$ per cent. in the adult. ${ }^{1}$

Digestion.-The principal requirement of the young infant during his waking intervals is food. His powers of ingestion, of digestion, and of excretion must therefore be active from the first. He obtains his food by sucking - an act which is performed in two stages. First, the lips are hermetically applied to the sides of the nipple so that the openings of the milk-ducts lie within the mouth. Then the infant, by lowering his jaw, depresses his tongue and the floor of the mouth. A diminished atmospheric pressure is produced in the enlarged cavity, and by its help milk is drawn from the secreting tubules of the breast into the dilated ampullæ in the interior of the nipple. The second stage of the process is reached when the infant closes his jaws to compress the nipple between his gums. The milk then flows from the ampullæ into his mouth. The whole act is repeated rapidly, and when the mouth has become sufficiently filled the milk is disposed of by swallowing.

The muscular energy required to suck is expended chiefly in

${ }^{1}$ Vierordt's Daten und Tabellen, 1906, Jena. 
the first stage, and therefore if the infant is feeble he may be unable to draw the milk to the nipple. Sometimes a malformation, such as a hare-lip or a cleft palate, by preventing a diminished atmospheric pressure in the mouth, leads to the same result. In other cases the infant may be vigorous enough, but the mother's secretion may be scanty, and can be drawn into the ampullæ only with difficulty. Under each of these circumstances the nutrition of the child is likely to suffer.

With regard to the first of the digestive juices, we find that the infant, having no occasion to masticate, needs but little saliva. This secretion therefore remains small in amount until some months after birth, when solid food is first taken. Even from the first, however, the saliva is able to decompose starch, though this action can be of little or no service to the infant until he has been weaned. His digestion depends much more on his gastric juice. This is physiologically active at the time of birth, and therefore the gastric mucous membranes of still-born children may be acid in reaction. The proportion of hydrochloric acid secreted during infancy is probably less than in later years. Analyses have shown that two hours after a meal free hydrochloric acid is present to the amount of $O^{\prime}$ I per cent. Both pepsin and rennin are normal products of secretion, and in addition small quantities of lactic acid are found. The source of the last named is uncertain.

As is well known, the act of vomiting occurs on very slight provocation in infants, and indeed their "possetting" is strictly physiological, representing nothing more than the regurgitation of surplus milk.

Having been subjected to the action of the gastric juice for some two to two and a half hours, the food is then passed on to meet the pancreatic juice. Little is known of the composition of this secretion in infancy. The fat-emulsifying ferment, steapsin, is stated to be present, and, in some cases, trypsin has been identified. With regard to the starch-ferment, amylopsin, most observers are agreed that it is absent. So far as is known to the contrary, the pancreatic juice is not of special importance in the earlier periods of life.

Although the pancreas thus appears to possess no great 
activity, the intestines undoubtedly play a leading part in digestion and in assimilation. In the first place, the secretion of the small intestine contains a ferment with a specific hydrolytic action on lactose. This ferment, which in the absence of amylopsin is the only one with power to form dextrose, is the infant's sole means of digesting the carbohydrate of milk. Second, the importance of the infant's intestinal tract in assimilation is indicated by its relatively long course. Whereas in adults the intestines measure five times the length of the body, in infants the proportion is as 7 to $\mathrm{I}$. Further, the villi are relatively more numerous in early life. On the other hand, the muscle-walls are relatively thin.

The bowels are relieved on an average once or twice a day. The process of defacation in infancy is peculiar, though its mechanism appears not to have been generally recognised. An infant, unlike older children and adults, expels his fæces by the unaided action of the rectum and its associated muscles. $\mathrm{He}$ makes no use of the muscles of inspiration, or of the muscles of the abdominal wall. As he does not need to increase the intraabdominal pressure, defæcation is a relatively easy and simple process. Quite early in childhood the mechanism undergoes a change, and the adult action is acquired-often, however, with the effect of prolapsing the rectum.

The physical properties of an infant's fæces are pretty constant. Of a light yellow colour, they possess a soft pasty consistency and an odour which is not disagreeable. Even in health they contain mucus and soft fragments of a white colour, which are probably small particles of casein that have escaped digestion.

Circulation and Respiration.-Before considering the physiology of circulation and respiration, we must turn aside for a moment to discuss a matter which is of cardinal importance in the physiology of the young. This matter is the relation between body-weight and body-surface.

We know that the volume of any given mass increases as the cube of its dimensions, but that its surface increases only as the square. Therefore the smaller the volume, the larger relatively is the surface. Applying this fact to animate masses, 
we find that small animals have relatively large surfaces, and that the same holds good of infants and children as compared with adults. The smaller-and therefore in many cases the younger-the child, the more extensive relatively will be the surface he presents to the surrounding air, and the greater will be his relative loss of animal heat. By way of compensation, and in order to maintain his temperature at a more or less equable level, the child must produce a larger amount of heat. But this necessitates an increased activity of metabolism, which in its turn demands a quicker circulation of blood, and therefore a more rapid heart-beat. We shall find, therefore, that the pulse is quicker in children than in men. It is generally quicker in short men than in tall. It is invariably quicker in small animals than in large-a mouse's heart beats 670 times a minute to a rabbit's I 50 times, and an elephant's twenty-five times a minute.

Turning now to the special features of the circulation in early life, we find that the maximum rate of heart-beat is in infancy. At birth it averages about I 30 a minute. By the end of the first year it has fallen to IIO; in the fifth year it is IOO; in the eighth year 90 , and in the twelfth year 80 . It is interesting to note that the rate at birth is not always the highest. Sometimes the pulse of an infant of three months is faster than it is in any of the earlier weeks. This rise is due to the greater activity of muscular movement at the latter age. Though the heart-beats are customarily expressed in relation to the age, this factor has no direct effect on the pulse, which depends, as we have seen, on bodily size.

The circulation-time-that is, the time taken by any given drop of blood to traverse the arteries, capillaries, and veins, returning to the point whence it started-is of course shorter the smaller the child. At birth the time is 12 seconds, at two years 15 seconds, and at fourteen years 15 seconds, as compared with 22 seconds in adults. Estimates of the relative amount of blood present at different ages, show that one-nineteenth of the body-weight of the young infant is thus accounted for, while one-thirteenth is the proportion in adults.

Another important relation is that between the volume of 
the heart and the cross-sectional area of the arteries. According to Landois, the child begins life with blood-vessels which are larger relative to the heart than at puberty or later. At birth the numerical ratio is given as $25: 20$, while at puberty it is $50: 140$, and in adults $290: 6 \mathrm{I}$. This striking increase indicates that the work of the heart is relatively less in early life, and it implies that the blood-pressure also is lower at that age.

Like the pulse-rate, the respiration-rate is fastest at birth. Beginning at 40 a minute, it has settled down to 30 by the end of the first year, to 25 by the fifth year, and to 20 by the sixteenth year. This gradual slowing is another effect of the ratio between volume and surface and its influence on heatloss. All warm-blooded animals share in this effect, so that we find that while the rat breathes 210 times a minute, the lion breathes ten times, and the rhinoceros six times.

Some difficulty arises in making an exact estimate of the respiration-rate in infancy, owing to the irregularity of rhythm that is characteristic of this age. Not only are the intervals between the breaths often unequal, but the special type of breathing known as Cheyne-Stokes breathing, which is pathological in adults, is physiological in infancy. This CheyneStokes breathing consists of periods of increasing frequency and amplitude of respiration alternating with periods of slow, shallow breathing that culminate in complete inhibition, and this may often be observed in infants even during their quiet sleep.

As might be expected, the function of the respiratory centre is less readily destroyed in the new-born than in older children, in whom it has become delicately susceptible to changes in the gaseous composition of the blood. This difference has been well illustrated experimentally in the case of animals. Full-grown guinea-pigs, for example, if immersed in water, are asphyxiated in from three to three and a half minutes, but new-born guineapigs can survive the want of oxygen for four times as long.

Kidneys.-Passing next to the excretory functions of the kidney, we find that they are in full action shortly after birth. While less than 50 c.c. of urine may be voided during the first day, the excretion by the third and fourth days may be five or six times as much. The relative amount is greater in infancy 
than in later life, but the absolute figure depends, as in the adult, on the amount of fluid taken into the stomach, and on the activity of perspiration. It has been estimated that for every 100 c.c. of milk taken, over 60 c.c. of urine is passed.

The composition of an infant's urine and the total daily amount of its solids are known with sufficient accuracy, in spite of the practical difficulty presented by the fact that an infant empties his bladder some twenty times in the twenty-four hours. The urine, which is acid in reaction, is at first intensely yellow in colour, and of a specific gravity averaging IOIO. It is cloudy from the presence of cellular débris and precipitated urates. A few days later, it becomes pale and clear, until it is almost colourless, and its specific gravity falls to I002 to I004. The amount of urea is small, but the uric acid is increased. The inorganic compounds present are very similar to those found in adult urine. A special constituent of urine during the first week, or even longer, is albumin. This albuminuria of the newborn infant is strictly physiological.

Excretion of Sweat and of Carbon Dioxide.-Of the remaining channels of excretion, little need be said. The relative amount of insensible perspiration is greater in infants than in adults, being about I.5 grm. per kilogramme body-weight per hour, as compared with 0.8 in man. ${ }^{1}$ The former figure, however, varies greatly with the restlessness and the muscular activity of the child.

With regard to the lungs, the excretion of carbon dioxide is greater per kilogramme body-weight the smaller the child. The respiratory exchange-that is, the intake and output of oxygen and carbon dioxide respectively-is, in infants, as in all small mammals, greater than in the bulkier adult man. This exchange, as Richet shows, bears a constant relation to the surface of the body.

Lymphoid Tissue.-Before concluding this section, we may briefly mention the physiological resources by which an infant endeavours to protect himself from infection by bacterial diseases. We have already learnt that the fœtus in utero is guarded against this danger by the natural and the acquired

1 Camerer in Pfaundler and Schlossmann's Diseases of Children, vol. i., p. 373 . 
immunities of the mother. After birth, however, the child is brought face to face with the pathogenic influences of his environment. $\mathrm{He}$ is compelled to depend on such powers of immunity as his mother has bequeathed to him, and when these are of no avail, he must fall back on his own defensive mechanism. He then relies largely on his lymphatic glands, and on his other collections of lymphoid tissue. These structures are well developed from early infancy.

In certain regions of the body very frequent demands are placed on their function, and they are specially liable to become enlarged. This change is most frequent in those glands which, being in direct functional relation with mucous membranes, are most intimately connected with the environment of the child. On this account the lymphatic tissue about the mouth and nose very commonly hypertrophies in order to offer a greater protection from septic processes in the neighbourhood. In this way we get enlarged tonsils, adenoids, and cervical glands. Similarly, the entry of pathogenic organisms from the lungs and from the intestines is opposed by the bronchial and the mesenteric glands respectively, which as a result very frequently increase in size.

\section{The Physiology of Metabolism, including}

\section{Animal Heat and NutRition}

With regard to the precise features which are special to the metabolism of infancy, much remains to be learnt. Our knowledge is derived from experimental investigations, but these are particularly difficult to make on infants. On the one hand, the intake of water, food, and oxygen must be estimated; on the other, the output of carbon dioxide, water, and nitrogenous excretions. In order to carry out these investigations, an infant must necessarily be subjected to a good deal of discomfort, as he must be enclosed in a special chamber. Restlessness and crying are the common results, and the normal metabolism is at once upset. To avoid these difficulties, many investigations have been carried out by the "statistical" method. The infant is permitted to live his ordinary life, without being shut up in 
any apparatus. The amount and the composition of his feeds are ascertained by regular weighing and analysis; the same is done to his urine and fæces; while his gain in weight is recorded. This method does not include any direct estimation of the intake of oxygen, or of the output of carbon dioxide and aqueous vapour by the lungs and by the skin. These values are calculated approximately from the other figures.

An important result of these investigations has been to show that, of the relatively large amount of food consumed by an infant, only a small proportion is required for his growth. Over 86 per cent. of his food is oxidised and eliminated through the lungs and the skin. This amount represents more than 80 per cent. of the total energy introduced by his food. Therefore less than 20 per cent. is retained for purposes of growth. ${ }^{1}$ Here is a fact of the greatest interest and importance. The belief is commonly held that the relatively large amount of food taken by an infant is required to further his growth, yet we can now see that this opinion is erroneous. Why, then, does an infant consume more than four times as much food as he requires for his development? How does he utilise all this apparent superfluity of energy?

We must, of course, accept the fact that he would not secure this large supply unless it was essential to his welfare. We also know that energy is indestructible, and that the amount taken into the body but not built up into living tissue must be employed in some other service. There are three principal channels in which it is turned to account. Some of it is used up in the muscular and glandular activities associated with digestion. Another portion is allotted to the contractions of voluntary muscles, of the heart, and of the diaphragm. The amount thus utilised will be least in early infancy, when the child is for the most part asleep and his voluntary muscles inactive. The largest proportion of all is lost in the form of animal heat. We have already learnt that infants, and indeed all children, lose much energy of this kind from their skin-by radiation, by convection, and by evaporation of sweat-and from the lungs.

1 Pfaundler and Schlossmann, op. cit. 
The question of heat-regulation in early life is therefore of great importance. It presents certain interesting features, because of the relation between mass and surface. Vierordt has shown that the cutaneous surface of an infant possesses nearly thrice the relative area of that of an adult. With this large surface exposed to surrounding media, a correspondingly large loss of heat is inevitable. The young of some animals have a slight advantage over infants, in that they are clothed in fur or in feather. Truly, infants have the protection of their garments, but neither clothing nor fur compensates for the large surface area. The only efficacious means by which the heatloss can be made up is by an increased production; and it has been shown experimentally that this production in animals is proportional to their surfaces. This holds, for example, of dogs of different sizes, and Langlois has established its truth for children of different sizes. Heat-production, therefore, must be most vigorous in infancy, less vigorous in later childhood, and least vigorous in maturity.

The physiological problem that has to be solved by any new-born, warm-blooded animal is, however, not merely that of heat-production, but of heat-regulation. In order that an equable temperature may be maintained through varying degrees of atmospheric temperature, production and loss must be very nicely balanced. To the full-grown animal, the problem has long since become simple; and a man will not vary a degree in temperature as he passes from the equator to the Arctic circle. But the new-born infant is at first unable to effect this accommodation. Accustomed throughout fœetal life to a medium that is almost constant in temperature, he is suddenly placed at birth under very different conditions. For a short while after his delivery his temperature is higher than that of his parent, but it soon falls, and for some time remains largely dependent on exposure to cold or to warmth. This instability is due, not to his greater extent of cutaneous surface, but to the imperfect development of his power of heat-regulation. In this respect infants are like the young of many other mammals. Pembrey has shown that the gradual development of the mechanism of heat-regulation can be traced by experiments on 
new-born animals. Thus, an adult mouse is able to respond at once to changes in external temperature, but if a new-born mouse is exposed to a lowered temperature, its body temperature quickly falls. Gradually, however, the young animal becomes better able to accommodate itself to such changes, and by its ninth day its mechanism works efficiently. Similarly, puppies and kittens are unable to respond to the changes in temperature. Young guinea-pigs, on the other hand, start with their heat mechanism in efficient working order, and they are able to maintain their temperatures at a constant level from the first. Two classes of new-born animals can therefore be recognised-those born with and those born without the power of responding to the external temperature. Infants belong to the second group. Each day, however, their inability is less marked, and finally they are able to keep their temperatures at one level. Yet throughout the whole of infancy, and for some years later, their temperatures will remain far less stable than in adults, and it will be elevated by so trivial a cause as crying.

The average temperature of an infant is very slightly higher than at subsequent ages. From infancy to puberty the drop is from one-tenth to two-tenths of a degree C. A further and equal fall occurs between puberty and middle age.

Nutrition.-The physiological sequence which we have now traced-namely, large surface area, excessive heat-loss, increased heat-production-must now be carried one step further. We must consider the subject of the nutrition and the food requirements of the child who, by virtue of his small size, is compelled to generate so large an amount of animal heat. This brings us back to our question why an infant consumes more than four times as much food as he needs for his growth. The excess is needed to satisfy the demands of heat-regulation. In the child appetite waits on surface area. Herein lies the clue to the whole subject of infant feeding, as well as of the feeding of older children. The heat-producing or calorific qualities of a child's diet are all-important to his welfare.

It has been established by experiment that the caloric requirements per kilogramme body-weight are nearly thrice as large in an infant as in an adult. Now, of the three classes of 
food-stuffs-protein, carbohydrate, and fat-the last named produces the most heat in its oxidation, and the first named the least. We would expect, therefore, that an infant would require relatively more of the heat-forming food-stuffs. Camerer has shown that while the consumptions of protein by the infant and by the adult are approximately equal per kilogramme bodyweight, the consumption of carbohydrate is as II : 5, and of fat as $4: \mathrm{r}$. Seeing, further, that the heat is lost largely by evaporation of water from the skin, we should expect to find a larger consumption of water by the infant. The ratio under this heading is $4: \mathrm{I}$.

Of the different food-stuffs that are used in the body for purposes of growth as opposed to purposes of heat-production, protein figures highest, 40 per cent. of the total being retained. Fat is deposited to the extent of 12 per cent. of its total consumption, but the carbohydrate (milk-sugar) is completely oxidised, and is excreted as carbon dioxide and water from the lungs, the skin, and the kidneys. Mineral ash is retained to the amount of 50 per cent.

These facts have their bearing on the infant's respiratory quotient,-the ratio between the amount of carbon dioxide expired and the amount of oxygen inspired, $-\frac{\mathrm{CO}_{2}}{\mathrm{O}_{2}}$. With a pure carbohydrate diet, the quotient must necessarily be $\frac{I}{I}$, since all carbohydrates contain hydrogen and oxygen in the exact proportions to form water, and their carbon is left to unite with oxygen taken in by the lungs; the resulting carbon dioxide that is expired must equal in volume the oxygen that has been inspired. With a protein diet the quotient is less (about 0.8 ), and with a fat diet it is least of all (0.7). Estimates of the quotient in infants give a high value-namely, 0.9. This figure must be interpreted as showing that an infant oxidises a very considerable amount of carbohydrate food.

From the fact that only 40 per cent. of the protein, 12 per cent. of the fat, none of the carbohydrate, and 50 per cent. of the salts taken in by the mouth are set aside for growth, we can draw the interesting conclusion that an infant may continue to 
grow even though he is insufficiently fed. The supply of food may be enough to satisfy the demands of growth, although it cannot supply the requisite surplus for heat-production. Under these circumstances his store of body fat will be drawn on to make good the deficit. When, however, it has been depleted, growth must come to an end. We may again observe that because an infant is increasing in weight he is not necessarily growing. Perhaps he is only becoming fatter. Both these observations are of practical importance, when examining records of weight and length, as an index to an infant's progress.

With these preliminary points before us, we can proceed to the important question of diet in infancy and childhood. It is the almost invariable custom, when treating of the feeding of infants and young children, to estimate the quantities of food by the age of the child. This method, though doubtless convenient, is neither scientific nor exact. It erroneously assumes that the age of an infant is the criterion of his nutritional requirements. A moment's reflection will show that the amount of food needed will depend rather on the size of his body than on his age. Infants of one age are by no means of one weight. Even at birth they may vary between very wide limits, as is shown in the following table by Quetelet and Altherr of the weights of some 600 infants :-

Weights of Infants at Birth.

\begin{tabular}{|c|c|c|c|c|}
\hline \multicolumn{3}{|c|}{ Weight in kilos. } & Number of Infants. & Percentage of Infants. \\
\hline $\begin{array}{l}I \\
I \cdot 5 \\
2 \\
2 \cdot 5 \\
3 \\
3 \cdot 5 \\
4 \\
4 \cdot 5\end{array}$ & $\begin{array}{l}\text { to } \\
" 1 \\
" 1 \\
" 1 \\
" \\
"\end{array}$ & $\begin{array}{l}1 \cdot 5 \\
2 \\
2 \cdot 5 \\
3 \\
3 \cdot 5 \\
4 \\
4 \cdot 5 \\
5\end{array}$ & $\begin{array}{r}2 \\
8 \\
54 \\
180 \\
251 \\
88 \\
15 \\
1\end{array}$ & $\begin{array}{r}0.33 \\
1.34 \\
9.01 \\
30.05 \\
41.90 \\
14.69 \\
2.51 \\
0.17\end{array}$ \\
\hline
\end{tabular}

It would obviously be absurd to maintain that, because all these new-born infants were of an age, therefore they should receive the same amounts of food. The criticism is applicable to each successive age of childhood.

We must be guided in this matter rather by the weighing- 
scales than by the calendar. Yet even the weight alone must not be accepted as a sufficiently accurate index, because it can easily be shown-the point is within everyone's experiencethat a child of, say, 8 kilos. does not require double the food of a child of 4 kilos. From the two facts-that a definite ratio exists between body-weight and surface area, and that 80 per cent. of the total energy derived from the food is lost as heat, the remaining 20 per cent. being stored up for purposes of growth-we can ascertain with physiological exactness the quantity of food that is essential to any particular child. (I) By first taking his weight, and from that figure calculating his surface area per unit of weight, we obtain the ratio between weight and surface. Numerous physiological experiments have provided us with data for estimating the loss of heat whatever the value of this ratio, and therefore we can ascertain the total heat that is lost. (2) The amount of milk or other food that is required must possess this calorific value, plus another 20 per cent. for growth. The calorific value of milk is readily ascertained from its composition. We therefore know what quantity of milk is needed by an infant of given weight.

This method is the only one that is in accordance with physiological teaching, and, for this reason alone, should supersede the rough-and-ready estimates that are too commonly employed. Fortunately, the method possesses the additional advantage that it is easily applied in practice. A short table can be constructed on the above lines, showing the amount of food required by children of different weights. This table can be cast in a form simple enough to be carried in the head without difficulty, and to be a practical guide to the dieting of any infant whose weight is known.

The principles on which this method is based will be best understood if we apply it, step by step, to infants from 3 kilos. to Io kilos. weight. ${ }^{1}$

(I) The surface area of an individual, whether child or adult, whose body-weight is known, can be calculated by the help of the formula :-

$$
\mathrm{S}=c \sqrt[3]{\mathrm{W}^{2}}
$$

1 I kilo. (1000 grms.) $=35$ oz. or, approximately, 2 lb. avoirdupois. 
where $S=$ the surface area in square centimetres, $W=$ the bodyweight in grammes, and $c$, a constant, $=\mathrm{I} 2 .^{1} \quad$ The following table illustrates its use. The average body-weight for each of eight age-periods is given in the first line. Next the surface area in square decimetres-as ascertained by the formula-is stated, while in the last line the number of square decimetres per kilogramme body-weight is recorded:-

Table showing the total Surface Area at cach of eight Ageperiods, from Birth to Maturity, and the Surface Area per kilogramme Body-weight.

\begin{tabular}{|c|c|c|c|c|c|c|c|c|}
\hline & Birth. & $\begin{array}{c}5 \\
\text { months. }\end{array}$ & $\begin{array}{c}1 \\
\text { year. }\end{array}$ & $\begin{array}{c}2 \frac{1}{3} \\
\text { years. }\end{array}$ & $\begin{array}{c}5 \\
\text { years. }\end{array}$ & $\begin{array}{c}10 \\
\text { years. }\end{array}$ & $\begin{array}{c}15 \\
\text { years. }\end{array}$ & Alult. \\
\hline $\begin{array}{l}\text { Weight in kilos. } \\
\text { Surface area in } \\
\text { sq. dcm. }\end{array}$ & 3.4 & 6.8 & 10.2 & 1 3.6 & I 7.5 & 28.5 & 46.5 & 70.0 \\
$\begin{array}{c}\text { Surface area in } \\
\text { sq. dcm. per } \\
\text { kilo. }\end{array}$ & 7.9 .12 & 43.08 & 56.40 & 68.28 & 80.88 & I I I.96 & 1 55.16 & 203.76 \\
\hline
\end{tabular}

The table shows clearly how much more extensive is the heatlosing surface of an infant at birth than that of an adultnamely, as $7.98: 2.92$, or nearly $3: \mathrm{I}$, the ratio stated in an earlier page.

Our interest, however, is not in the age-factor, but in the weight, and the next table gives the figures for infants of each successive kilogramme from 3 to Io inclusive :-

Table showing the total Surface Areas of Infants from I to Io kilogrammes Body-weight inclusive, and the Surface Areas per kilogramme Body-weight.

\begin{tabular}{|r|r|r|r|r|r|r|r|r|}
\hline $\begin{array}{r}\text { Weight in kilos. } \\
\text { Surface area in sq. }\end{array}$ & 3 & 4 & 5 & 6 & 7 & 8 & 9 & I0 \\
$\begin{array}{r}\text { dcm. } \\
\text { Surface area in sq. } \\
\text { dcm. per kilo. }\end{array}$ & 24.96 & 30.24 & 35.04 & 39.60 & 43.80 & 48.00 & $5 \mathrm{I} \cdot 96$ & 55.68 \\
\hline
\end{tabular}

1 The value of $c$ has been variously estimated. Meeh takes it as 12.3; Lassauer gives for infants, $c=10.3$; Rübner states that for mammals $c=11 \cdot 2$; Waller puts it as low as 10.5 . 
We have next to consider the rate of heat-loss in infants of different weights. Observations on this point made directly on infants are few in number, and the results are by no means concordant. On the other hand, many estimates for adults have been made, and these may be taken as the standard. The loss from an adult man at rest is given by Tigerstedt as 1680 calories in the twenty-four hours, or 24 calories per kilo. ${ }^{1}$ Since the losses from individuals of different weights are proportional to their surface areas per kilogramme body-weight, the above figure enables us to estimate the loss in infants of all weights.

From the last table but one, we see that a man of 70 kilos has a surface area of $2.92 \mathrm{sq}$. $\mathrm{dcm}$. per kilo. body-weight, and from the last table we find that for a new-born infant of 3 kilos. the corresponding figure is $8 \cdot 32$. Therefore the infant's daily heat-loss will be $24 \times \frac{8.32}{2.92}=68.4$ calories per kilo., or 205.2 calories in all. In the same way we can obtain the loss from infants at each successive kilogramme increase. The results are seen in the next table:-

Table showing the Heat-loss per kilogramme and per total Bodyweight in Infants from 3 to Io kilos. inclusive.

\begin{tabular}{|c|c|c|c|}
\hline $\begin{array}{c}\text { Weight of Infant } \\
\text { in kilos. }\end{array}$ & $\begin{array}{c}\text { Surface Area } \\
\text { in sq. dem. per kilo. }\end{array}$ & Heat-loss per kilo. & Total Heat-loss. \\
\hline 3 & $8 \cdot 32$ & $68 \cdot 4$ & $205 \cdot 2$ \\
4 & $7 \cdot 56$ & $62 \cdot 1$ & $248 \cdot 4$ \\
5 & $7 \cdot 00$ & $57 \cdot 5$ & $287 \cdot 5$ \\
6 & $6 \cdot 60$ & $54 \cdot 2$ & $325 \cdot 2$ \\
7 & $6 \cdot 26$ & $51 \cdot 5$ & $360 \cdot 5$ \\
8 & $6 \cdot 00$ & $49 \cdot 3$ & $394 \cdot 4$ \\
9 & $5 \cdot 77$ & $47 \cdot 4$ & 426.6 \\
10 & $5 \cdot 57$ & $45 \cdot 8$ & $458 \cdot 0$ \\
\hline
\end{tabular}

The last column, therefore, represents the calorific value of the food needed to make good this heat-loss. But the food thus employed constitutes only 80 per cent. of the total requirements, and the figures in this column must be increased by

1 The term "calorie" is here used as the large calorie-i.e., the amount of heat required to raise $\mathrm{I}$ kilo. of water through $\mathrm{I}^{\circ} \mathrm{C}$. 
20 per cent. We then obtain the following as the minimum calorific values of an infant's diet at different weights :-

Table showing the Minimum Calorific Vahes of the Food of Infants from 3 to 10 kilos. inclusive.

\begin{tabular}{|c|c|}
\hline An Infant weighing & Requires food to the value of \\
\hline 3 kilos. & 256.5 calories \\
\hline $4 \quad "$ & $310.5 \quad$ \\
\hline $5 "$ & $359 \cdot 4 \quad "$ \\
\hline $6 \quad "$ & $\begin{array}{ll}406 \cdot 5 \quad "\end{array}$ \\
\hline $8 \quad$, & $\begin{array}{l}450 \cdot 0 \quad " \\
493.0 \quad "\end{array}$ \\
\hline $9 "$, & $533.25 "$ \\
\hline to & $572 \cdot 5$ \\
\hline
\end{tabular}

(2) We have now to interpret these results in terms of quantities of milk. Let us consider first the quantities of human milk.

The composition of human milk is variable in all its constituents; but, on an average, IOO c.c. contain :-

$\begin{array}{llllll}\text { Fat . } & . & . & . & 2.75 & \text { grms. } \\ \text { Protein } & . & . & . & 2.0 & \text { " } \\ \text { Carbohydrate } & . & . & . & 5 & 0\end{array}$

Now, the heat-values of I grm. of each of these three substances is :-

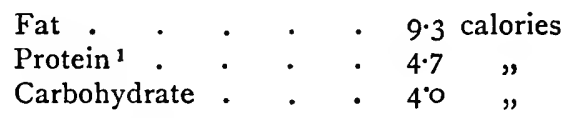

Therefore the calorific value of 100 c.c. of human milk is :-

$$
\begin{aligned}
& \text { Fat . . . . } 2.75 \times 9.3=25.575 \\
& \text { Protein . . . } 2.0 \times 4.7=9.4 \\
& \text { Carbohydrate. . } 5.0 \times 4.0=20.0 \\
& 54.975 \text { calories. }
\end{aligned}
$$

1 The full heat-value of protein is 5.5 calories per gramme, but as I grm. of protein forms $0.3 \mathrm{grm}$. of urea, which is excreted, the heat-value of the latter, 0.8 calorie per gramme, must be subtracted from the 5.5 . The corrected figure for protein is, therefore, as given in the text. 
In other words, I calorie of heat is provided by every 2 c.c. of human milk. Therefore we have the following as the minimum quantities of breast milk required by infants of different weights :-

Quantities of Human Milk required by Infants of Different Weights.

\begin{tabular}{|c|c|c|}
\hline An Infant of & Requires & Which is contained in \\
\hline $\begin{array}{rl}3 & \text { kilos. } \\
4 & " \\
5 & " \\
6 & " \\
7 & " \\
8 & " \\
9 & " \\
\text { 10 }\end{array}$ & $\begin{array}{l}256.5 \text { calories } \\
310.5 \quad " \\
359.4 \quad " \\
406.5 \quad " \\
450.6 \quad " \\
493.0 \quad " \\
533.25 \quad " \\
572.5 \quad "\end{array}$ & $\begin{array}{ll}513 & \text { c.c. of milk } \\
621 & " \\
719 & " \\
813 & " \\
901 & " \\
986 & " \\
1066.5 & " \\
1145 & "\end{array}$ \\
\hline
\end{tabular}

It remains to put these results in a form that can be easily remembered and readily applied in practice. They may be stated thus :-

Amount of Mother's Milk required Daily by an Infant of 3 kilos. (or $6 \frac{1}{2} \mathrm{lb}$.) is $\frac{1}{8}$ th his weight.

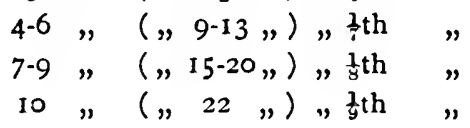

It is instructive to compare these figures, based on physiological experiment, with the average secreting capacity of a woman's breast. According to Goodhart and Still, "it has been estimated that the mother supplies to her baby about half to three-quarters of a pint [ 300 to 450 c.c.] in the twenty-four hours in the first week or two, and that this gradually increases until, in the later months of lactation, a daily average of about two pints [I 50 c.c.] is reached." 1 Pfaundler and Schlossmann give the following more precise figures. ${ }^{2}$ The average daily secretion at the end of the first week is 470 grms., by the middle of the twentieth week-when the average weight is, approximately,

1 Diseases of Children, 8th Ed., 1905, p. 38.

2 Diseases of Children, 1908, vol. i., pp. 347, 383, 39ז. 
6 kilos.-it is 900 grms. The average daily secretion of a good wet-nurse is given as 1000 to 1500 grms.

If the infant's food is to take the form of cow's milk, the quantities are determined as above. Cow's milk, like human milk, is variable in composition, but, on an average, IOO c.c. contain :-

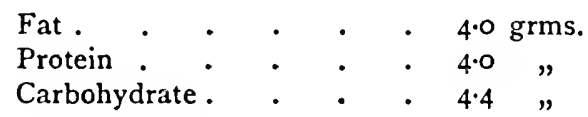

The calorific value of 100 c.c. is therefore :-

$$
\begin{aligned}
& \text { Fat . . . } 4.0 \times 9.3=37.2 \\
& \text { Protein . . . . } 4.0 \times 4.7=18.8 \\
& \text { Carbohydrate . } 4.4 \times 4.0=17.6 \\
& \overline{73 \cdot 6} \text { calories. }
\end{aligned}
$$

In other words, I calorie is provided by 0.75 c.c. of cow's milk. Infants therefore require the following amounts:-

Quantities of Cow's Milk required by Infants of Different Weights.

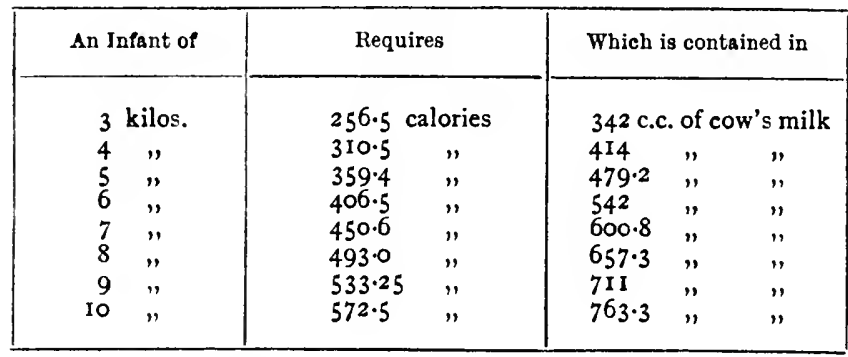

Expressing these results in the same form as those for human milk, we find that:-

The Quantity of Cow's Milk required by an Infant of

$$
\begin{aligned}
& 3 \text { kilos. (or } 6 \frac{1}{2} \mathrm{lb} \text {.) is } \frac{1}{9} \text { th his weight. } \\
& \text { 4-5 " ("9-1I } ") \text { ), 10th " } \\
& 6 \text { " ( } 13 \text { " ) " } \frac{1}{11} \text { th " }
\end{aligned}
$$

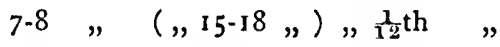

$$
\begin{aligned}
& \text { 9-10 " (,20-22, "), , theth " }
\end{aligned}
$$

Before we conclude this aspect of infant feeding, two or 
three additional points must be mentioned. The quantity required by an adult is influenced by the amount of muscular work that he will be called on to perform. In an infant this factor is of relatively little importance, because, until he is able to stand, the amount of muscular work he performs is not great. When, however, he begins to support his weight on his feet, to walk and to run, he needs a greater supply of energy. We must further realise that the larger the child, the greater is the mechanical disadvantage under which he labours when he attempts muscular work. As was shown by Herbert Spencer, ${ }^{1}$ an animal in doubling its height increases its volume as the cube of its dimensions, but its muscular power only as the square of the cross-sectional area of its muscles. In order to effect the same movements in the larger body, the muscles must have relatively greater transverse dimensions-that is, they must be bulkier. The consumption of energy will be proportionately increased, and the resulting greater demand for food must be satisfied. Another point that must be taken into account is the difference in temperature between the body and the atmosphere, since the greater this difference the more rapid will be the rate of cooling. In warm weather, therefore, less heat is lost and less food will be required, while the converse holds for cold weather. Sufficient allowance is not always made for these changes. Mothers, especially, view with apprehension the diminished appetite that is customary in infants and children in the hot months. Similarly, a child who is kept in a warm nursery cannot require as much nourishment as one who is taken into the open air on cold days.

Finally, we have to consider the frequency with which infants should be fed. From the fact that the respiratory exchange is relatively great in early life, we know that the oxidation of food-stuffs is rapid in infants. We may also conclude that the smaller the infant, the more rapidly will he suffer from want of food. Hunger is to him a more acute affliction than to larger children or to adults. He must never go long before his supply of fuel is replenished. On the other

1 Principles of Biology, 1898 , vol. i., p. I 5 I. 
hand, the amount of nourishment we can give him in any one feed is limited by the capacity of his stomach. The minimum number of times of feeding will therefore be the quotient of the volume of the daily milk-supply divided by the capacity of the stomach. The sizes of the stomachs of infants of successive kilogrammes from 3 to 9 inclusive are: 60, 90, 120, 140, I80, 225 , and 290 c.c. respectively. ${ }^{1}$ Allowing the undesirability of distending the stomach to its full capacity, we may discount these numbers by one-fourth, in order to obtain the suitable size of each feed :-

Number of Daily Feeds for Infants of Different Weights.

\begin{tabular}{|c|c|c|c|c|}
\hline $\begin{array}{c}\text { Weight } \\
\text { in kilos. }\end{array}$ & $\begin{array}{c}\text { Volume of Milk } \\
\text { consumed } \\
\text { in c.c. }\end{array}$ & $\begin{array}{c}\text { Capacity of } \\
\text { Stomach. }\end{array}$ & Size of Feed. & $\begin{array}{c}\text { Number of } \\
\text { Feeds. }\end{array}$ \\
\hline 3 & 513 & 60 & 45 & I I \\
4 & 62 I & 90 & 68 & 9 \\
5 & 719 & 120 & 90 & 8 \\
6 & 813 & I40 & 105 & 7 \\
7 & 901 & I80 & I35 & 6 \\
8 & 956 & 225 & I68 & 5 \\
9 & I056 & 290 & 218 & 8 \\
\hline
\end{tabular}

With regard to the feeding of children beyond the age of infancy, little more need be said. The same factors are equally applicable, but as a child becomes less dependent on the attentions of others, and better able to express his own wants, his dietary can be arranged with much less difficulty. Though his growth is now slower, a greater margin is required for the supply of energy for muscular exertion. The demand for food-stuffs of high calorific value is still imperative, and shows itself in the partiality for carbohydrate in the form of sugar and starchy foods, and for fat in the form of butter. Protein is desirable, and should be given as meat, though its amount need be only small. As a general rule, the natural tastes of a child should be fully consulted, instead of being, as is often the case, disregarded ; nor should the child be compelled to eat, or to abstain from eating, in accordance with the requirements or habits of adults.

${ }^{1}$ These figures are based on observations by W. Camerer, jun. 


\section{The Physiology of Puberty}

Even from early childhood differences appear between the two sexes, each as a rule outstripping the other in special directions. Muscular development is more marked in the boy, and therefore his bones must be correspondingly strengthened to withstand the increasing strains and stresses thrown upon them. His heart must beat more forcibly, the movements of his lungs must be freer, and his chest must become broader and deeper to afford increased space to these organs. With this change in his two vital functions, his general vigour increases, and his greater muscular power tends to promote those more dominating or dictatorial qualities of mind that later characterise the adult man.

The girl, on the contrary, with her muscular and skeletal tissues less developed, inclines more to a certain passivity of function. Her growth, both in height and in weight, is less rapid than the boy's, and even in proportion to her stature her weight is less. The vital capacity of her lungs is less, and her respiratory muscles are weaker. The lifting power of her back, legs, and arms is relatively less than in a boy. These differences must affect the development of her heart, which is not called on to perform so much muscular work. The girl, therefore, tends to a more physically restful mode of life. She is content to observe, where the boy must act. Her mental powers of association are more often exercised, and she soon leaves her brother behind in quickness and alertness of perception.

With the advent of puberty and the first appearance of the secondary sexual characters, the physiological distinctions between male and female become accentuated. These changes appear earlier in a girl. About the twelfth year, her rate of growth leaps forward, and, as we have seen, she soon overtakes the boy in height and in weight. By the thirteenth year she is both taller and heavier, but she maintains her lead only for two or three years, until, with the onset of puberty in the boy, his development is quickened, and he regains for good the advantage he has temporarily lost. 
It will not be necessary to consider here the various anatomical changes that make up the secondary sexual characteristics, as they are sufficiently well known. The physiological connection between these changes and the newly awakened activity of the sexual organs is, however, but little understood, and for the present we have to content ourselves with speculations as to supposed internal secretions of the testes and the ovaries, whereby the other organs of the body are modified. Ovulation and its accompanying cyclic phenomena remain unaccounted for by any convincing physiological explanation. We know, however, that, associated with this change, and perhaps as a result of it, profound alterations occur in a young girl's economy. Thus she expends even less energy than before by way of muscular contraction. Her food requirements become relatively small, and yet she shows an increased tendency to the deposit of subcutaneous fat. The specific gravity of her blood begins to fall. Changes such as these vaguely suggest some deep modification of her metabolism, but do little more than accentuate the darkness in which the physiology of puberty is surrounded. 


\section{CHAPTER III}

\section{THE PSYCHOLOGY OF CHILDREN}

InTRoDUCTION-Observations on Infant Psychology; Sensations the Basis of Mentality; Ideas; Recognition; Memory; Judgments ; Influence of Environment; Walking; Talking. AT BIRTH-Sleep; Hunger; Pain; Hearing; Sight; No Intelligence; No Emotions; No Power of Expression; Automatic and Reflex Muscular Movements. THE FirST MoNTH-Sleep; Impulsive Movements; Yawning; Hiccoughing ; Movements of Eyes and Eye-lids ; Taste and Smell ; Effect of Light ; Appreciation of Sound ; Pleasant and Unpleasant Feelings ; Smiling; Phonation; Mental Association. The Second, ThIRd, AND FOURTh Months-Reflex Movements ; Co-ordination of Eyes; Balancing Head; Sensations; Grasping Objects Seen; Hearing; Emotions; Fear of Strangers. The FifTh, Sixth, AND SEventh MoNTHS-Use of Arms; Interest in Toes; Grasping Movements; Muscular Sense ; Sitting Posture ; Pleasure from Auditory Sensations; Expression of Feeling; Recognition; Memory ; Early Consciousness of Self.

THE mental development of children has been made the subject of scientific investigation only in recent years, and its study forms a comparatively young branch of psychology. The pioneer efforts of Darwin, who in 1877 published a biographical sketch of an infant, ${ }^{1}$ were followed by the more elaborate work of Preyer, ${ }^{2}$ who recorded in detail the progress of his son up to his fourth year. These observations have been confirmed and amplified, and the labours of Romanes, ${ }^{3}$ Baldwin, ${ }^{4}$ Shinn, ${ }^{5}$ Sully, ${ }^{6}$

1 Mind, July, I877, pp. 285-294.

2 Mental Development in the Child, IV. Preyer, 1901; The Development of the Intellect, W. Preyer, 1906.

3 Mental Evolution in Man, G. R. Romanes, I 888.

${ }^{4}$ Mental Development in the Child and the Race, J. M. Baldwin, I895.

${ }_{5}^{5}$ Biography of a Baby. 'S Studies of Childhood, James Sully, I903. 56 
Major, ${ }^{1}$ and others, have provided us with an accurate survey of a child's mental evolution. This knowledge has a twofold value. It has traced the course along which an infant's intelligence naturally progresses, and has thus mapped out many of the foundations upon which the education of children should be planned. Again, the essentially synthetic character of the study has supplemented our understanding of adult psychology by bringing a touchstone to a science which is wholly analytical. The enormous intricacy of adult consciousness and its evervarying complexions envelope the analysis of its mental states in a mist which only the keenest eyes can penetrate. In the infant the problem is incomparably simpler, for the mind of a new-born babe is a blank sheet on which the simple lines, at first marked laboriously one by one, and later, combined and intersected, may be studied accurately and interpreted with ease.

Infants at birth have their intellectual powers narrowly limited in every direction, but their potentialities are great, and the unfolding of their minds depends more on their own experiences than on any other factor. The steps in this development, and the order in which they appear, are the same in all children, but the duration of each stage varies with the individual. Too much value must not be set on the particular week or month in which some new mental attribute is first disclosed. In this chapter and the next, which will be concerned with the psychology of children as a class, and not with the mental growth of one particular child, this variability will be recognised, and advances in intelligence dated only approximately.

Before embarking on a detailed account of the psychological growth of normal children, we must take a preliminary survey of the subject. The basis of all mentality is Sensation, which is itself dependent on stimulation of sensory nerve-endings. It therefore follows that at the moment of birth an infant can possess little or no capacity for executing even the most rudimentary cerebral process. The organs devoted to sight, hearing, smell, taste, pain, and perception of heat and cold ${ }_{1}^{1}$ First Stcps in .Mental Growth, D. R. Major, 1906. 
have never yet been used, while the tactile and muscular feelings which have arisen in utero, have been of too vague a character to awaken mental response. After birth, stimulation of every sense is of frequent occurrence, and the infant gradually begins to show an appreciation of the resulting sensations. He thereby gives the earliest proof that he has come into possession of his primitive Ideas or Percepts. Of equal importance is the fact that from the first the natural response to all stimuli is by way of Muscular Action. To estimate the influence of movement on the future development of the child, we need only recall the part played by movements of the eyes in seeing, of the arms in touching and holding, of the legs in walking, and of the larynx in speaking.

In the course of time, the infant begins to grasp certain sensations as part of his past experience. Frequent repetition makes them familiar, and he develops the power of Recognition. An idea, provoked by a stimulus, is presented to his mind, and revives "supplements" which are the products of past sensations. At the same time, the association of the new idea with its centrally-aroused supplements produces a mood of familiarity, and he recognises the object causing the new idea. This marks an important advance, and leads in due course to the awakening of Memory. Here we have something more than mere recognition, for, with memory, the idea which calls up centrally-aroused supplements is itself centrally aroused.

As soon as the infant has developed the two faculties of recognition and memory, he has obtained a new and powerful lever. He can raise himself to institute comparisons and to establish contrasts. For a long while now, such of his waking hours as are not taken up with feeding are occupied almost exclusively with attempts to regard his new experiences in the light of the past, and to discriminate between what is strange and what is familiar. These efforts require the formation of Judgments, and are of cardinal importance in helping the infant to enlarge his intelligence. For a long time only broad resemblances and fundamental differences are appreciated, and all points of detail are completely overlooked. Gradually, though only with long practice, discrimination becomes more 
accurate, judgments more complex, and the faculty of Reasoning assumes its pre-eminent position.

Pari passu with these intellectual changes, the emotions expand, and by facial expression, voice, and gesture the infant begins to reveal the growing complexities of his moods.

Seeing that this mental growth is dependent on a child's own experiences, we must at once admit the importance of environment. The wider and more varied this is, the more quickly is the child likely to progress. We are therefore able to understand the very deep effect that ability to walk exerts on the growth of the mind. So long as the child is compelled to remain on the spot where he has been placed, his experiences, which at this time are largely tactile and muscular, must be limited to an environment measured by the length of his arms. But make locomotion possible, and new worlds are opened. Later, speech is added to the mental equipment. The gap between the new understanding and that of a few months before is rapidly widened, and the child is soon placed on a level higher than that of the most intelligent animal. Many years, however, filled with experiences must pass before the young mind can put itself in true relation with its surroundings; but every future advance will depend on the same factors as before. They are reasoning and memory, which are built up of ideas that are founded on sensations, themselves the result of stimulation of sense-organs.

From this summary we can now turn to a more detailed study, tracing the progress step by step. To follow these changes to the best advantage, we must first form a clear idea of the earliest state of the mind whose development we are to consider. We will begin, therefore, with the mental capabilities and limitations of a new-born infant.

At Birth.-The day has fortunately long since passed when every child was supposed to be born with a millstone of original sin round his neck. We now know that he comes into the world a made-up sample of his ancestry, with merely potential capacities for good and evil. We accept his obvious relations to the young of other animals, and we are able to analyse his simple mental organisation. At first he spends the greater 
number of his hours in sleep. His life in the womb has afforded him no experience of those sensations which alone can maintain consciousness. He is quickly tired by the new stimulation of his sense-organs, so that he falls again into the sleep in which he has hitherto been wrapped. From this quiescence he awakes from time to time in response to various stimuli. Of these, the most certain in their action are hunger and pain. The periodic influence of the former rouses him at regular intervals, and keeps him awake until the comfort of repletion as regularly puts him to repose again. Pain, whether due to unsatisfied hunger or to other causes, is the most potent stimulus of all, and is able to maintain wakefulness for hours together. Compared with these influences, light and sound have but little effect, and so commonly are they passed by unnoticed, that the new-born infant has been held incapable of seeing and hearing. This statement is true to the extent that no sign is made to show that a stimulus from either source is appreciated, yet it is probable that the deficiency lies rather in expression than in sensation. The anatomical and histological characteristics of the organs concerned are not known to change in the first week of life, yet both light and noise are appreciated before the end of this period. Donders ${ }^{1}$ recorded his observation of an infant who, within a few minutes of birth, not only stared fixedly at an object held before him, but was seen to follow its movements with his eyes. Major ${ }^{2}$ states that his child, one hour after birth, was seen to close his eyes in response to the stimulation of a bright light. Both seeing and hearing are, however, of subordinate importance immediately after birth, and in their stead the senses of taste and smell are relatively acute. In consonance with this we find that, in the lips and tongue, feelings of touch, heat, and cold are well developed.

Beyond these limited capacities, a new-born infant possesses but few powers. With no experience, he has neither intelligence nor emotion, no appreciation of self, and no muscular sense. ${ }^{3}$ $\mathrm{He}$ is incapable of expression by movements, looks, or sounds.

1 Archiv. für Ophthalmologie, Bd. 17, 1871, p. 34.

2 First Steps in Mental Growth.

3 The sense by which we know the position of our limbs. 
Truly, crying is among his earliest acts, but this is to be regarded as a reflex not yet attaining to the level of an expressive utterance; for children born without brains will cry as readily as normal children and on the same provocation. With regard to muscular action, the only movements that are made belong to one of two groups. They are either automatic -random and purposeless motions of the limbs-or reflex, as in sneezing, coughing, or sucking. A reflex act that deserves special notice is that of clasping the fingers round any object, such as a pencil, that is allowed to stimulate the skin of the palm of the hand. The unusual strength of the muscular response is shown by the well-known experiment, first made by Dr Louis Robinson, of inducing a new-born child to take hold of a rod from which he is then allowed to hang. The grasp is sufficiently powerful to support the entire weight of the body. Movements on a higher level-instinctive and voluntary-have yet to make their appearance, and for the present there is no power of co-ordinating the muscular contractions necessary for steady or purposeful movements.

The First Month.-With these primitive endowments the infant begins at once to enlarge his powers, though in the first month-the period we shall next consider-he leads an automatic life. Sleep continues to fill the greater part of his days, and at all times the bodily attitude of greatest comfort is that to which the child has been accustomed in the narrow limits of the womb-the arms folded upon the chest, the legs bent over the abdomen. The instinctive movements of stretching after waking appear in the first week or two, and the reflexes of yawning and of hiccoughing may occur even earlier. Though the power of grasping with the fingers is readily shown, that of opposing the thumb-an action which gives man his supremacy over animals-does not appear for three or four months. The eyelids can be opened and shut, but the eyes themselves move irregularly, without associated action. They often turn in contrary directions, so that squinting is frequent.

On his sensory side the infant shows appreciations which are almost exclusively those of a gourmet. The co-adjutant 
senses of taste and smell being well developed, the predominant sensations in consciousness are those derived from tongue and nose. His food soon becomes the absorbing interest. Indeed it may be said that the infant's mouth is his world, and his faucial pillars his pillars of Hercules. Not only is taste so keenly developed that substituted foods with unnatural flavours will be refused, but the appreciation of temperature is acute, both in the lips and tongue. He may summarily reject liquid that is either too warm or too cool. As a rule, an infant wakes only to be fed, and his periods of consciousness are largely filled with cognate sensations. The progress of those senses which are not associated with feeding is therefore slow, though some development in seeing and hearing is noticeable within the first few weeks. At this age infants will gaze steadily at moderately bright daylight, but very strong light is avoided. Shining, metallic objects, and artificial illuminants, especially candles, are regarded for lengthy periods, and before long cause movements of the eyes as they travel from place to place. The indifferent appreciation of sound that may be present from the outset becomes sharper, and within the month the child may be seen to start at loud noises. Even earlier than this, however, the influence of soft noises is apparent in the soothing effect of the mother's voice.

The earliest indications of feeling, both pleasant and unpleasant, appear within a few days of birth. As a general rule, pleasurable feelings result from moderate stimulation of one or other sense-organ; unpleasant feelings are the outcome either of the reverse condition-over-stimulation of a senseorgan-or of a sudden interruption of a pleasant experience. The dominant feelings are naturally associated with food. Hunger and satiety are the alternating influences, the former giving rise to fretfulness, crying, and vigorous impulsive movements, the latter to content, quiet, and sleep. When Major's infant was being suckled on the second day, the nipple temporarily escaped from his lips, and the child whimpered. ${ }^{1}$ Preyer's infant gave a satisfied smile of satiety after a feed on his tenth day. Apart from sensations connected with food, 
infants will show feeling arising out of other experiences. For example, they all dislike cold, and protest against wet clothing; they are unmistakably gratified by the warmth of a hot bath.

However, in his first weeks the infant seems without the power of expression, or, at any rate, the expression is restricted to a fleeting smile that indicates a general condition of wellbeing, and to the loud crying, which, as a rule, expresses hunger. Crying at this age is not associated with tears, and, as Darwin pointed out in his "Expression of the Emotions," these are not observed in crying until some time after the lachrymal glands have become active.

Speech, or rather phonation, may be regarded as making its most elementary beginnings in the first month, inasmuch as some vowel-sounds are uttered by chance as the child cries.

In the domain of intellect we have at present little to record. Memory and recognition have not yet appeared, though a certain degree of mental association may be possible. Thus Baldwin mentions ${ }^{1}$ how his infant, before the end of the first month, was taught to go to sleep by being placed face downwards, while his back was patted. Miss Shinn ${ }^{2}$ records an instance of association at the end of the first month, when her niece showed that she could discriminate between solitude and society. More usually the earliest associations are alimentary, and a hungry infant taken in his mother's arms to be fed may cease crying even before the breast is reached. In this example the sensations dependent upon the act of being placed near the breast are associated by the help of past experiences with the imminent gratification of hunger.

The Second, Third, and Fourth Months.-We have now followed the infant to about the end of the fourth week. In the next two or three months, his progress, especially in the higher forms of mental activity, is rapid. His muscular powers remain at first about the same, but there is an increasing tendency to substitute the higher forms of action for the lower. Movements of the arms are made less at random, and a single, definite movement, such as waving the arms or rubbing

1 Mental Development in the Child.

2 Biography of a Baby. 
one foot against the other, may be repeated over and over again. The reflex act of clasping with the fingers now appears as a conscious attempt to grasp objects, and in time the power of setting the thumb in opposition to the other fingers is attained. The asymmetrical movements of the eyes now become less frequent, until finally co-ordination is established; but until this complex power has become ingrained by frequent repetition, temporary lapses are not uncommon. As the eyes become more readily controlled, their movements are carried out more quickly, and the child may be able to follow the swinging of a pendulum or the flight of a distant bird. The eye-lids rise and fall regularly, and when the face is threatened by the sudden approach of an object, the child blinks. The range of action of the eyes is extended by lateral movements of the head, which, at first executed slowly and with evident effort, become more easily mastered, until the face can be promptly turned to look this way or that. A somewhat later and still more important development in muscular control is the power of balancing the head upon the shoulders. This act is one of considerable complexity, and requires not only a nicely graduated co-ordination of the many muscles of the neck, but a sense of position relative to surrounding objects. It is acquired, approximately, at the end of the third month, though it may be exercised in the second month, or be absent after the fifth month. ${ }^{1}$ It begins as briefly sustained efforts, and not until some few months later does the head cease to be "wobbly." Preyer ${ }^{2}$ lays stress on the first successful effort to hold the head erect, which act he instances as the earliest manifestation of will.

Sensory impressions now begin to awaken more interest. Glistening objects are particularly attractive, and even elementary differences in colour appear to be noticed, so that an infant will gaze with peculiar intentness at a brightly tinted curtain or dress. About the same time a co-operative association, which is destined to play a very important part in his future development, becomes established between the sense of sight

1 See p. 204 for delayed power of balancing the head as a sign of mental deficiency.

${ }^{2}$ Mental Development in the Child. 
and movements of the arms. A desire to grasp with the hand all objects seen by the eye rapidly grows, till it takes a firm hold of the child and occupies a large share of his waking hours. For the present, however, the powers of sight and of grasping are too rudimentary to afford- each other much mutual assistance, and the infant will as readily attempt to seize the electric light on a distant wall or even the moon looking in through the window, as the coloured ball hanging a few inches above his head. Such futile efforts result from the rudimentary state of the muscular sense, and knowledge in this direction comes only with experience. Until this has been obtained, the infant attempting to grasp a near object must clutch the air, until by chance his fingers happen to light on the desired article.

With regard to hearing, muscular responses to sound stimuli may have been noticed before the completion of the first month, and at a later date they become both brisker and more elaborate. Loud noises cause the child to start, and instrumental sounds, whether harmonious or discordant, may be listened to with pleasure. The tick-ticking of a watch is often particularly attractive. Gradually the infant begins to know the direction from which a sound has travelled, and though at first he may turn his head in mistake, he will, during the third month or so, learn to move it with increasing accuracy, and soon the localisation will be accomplished with ease.

Step by step, as sensations multiply, emotional states become more complicated. Feelings, pleasant and unpleasant, are no longer merely or mainly caused by moderate or excessive stimulation of sense-organs. They now result from the child's gratified or thwarted impulses. These states are induced, especially in connection with the infant's increasing desire to seize and to hold. If he is unable to reach the toy that has taken his fancy, or is deprived of the spoon clutched in his hand, his displeasure is apparent. On the other hand, let him grasp the toy or keep hold of the spoon, and he soon shows his pleasure. To express moderately unpleasant feeling, the infant shows a fretfulness of voice and action, and to indicate surprise, he begins to open his eyes wide. Laughing in response to tickling has been observed in the sixth week; but 
at this age it has rather the characteristic of an impulsive movement, and children do not usually laugh as an expression of emotion until a much later date. As their likes and dislikes become more clearly distinguished, they begin to turn the head away as a sign of refusal. The motion is usually first made in connection with feeding, and its evident purpose is to remove the lips from the undesired food. Expression of feeling by means of speech remains very elementary, though there is a tendency to employ certain sounds to indicate certain feelings. About the second or third month the first consonant is articulated, and in such sounds as "ma" or "pa," the first word is uttered.

At this period rapid progress is being made in the higher levels of mental development. The prominent feature is the growing interest which the infant takes in his surroundings; but this can be measured only by watching the movements of his head, eyes, and arms in connection with such visual and auditory stimuli as he experiences. The power of association has developed, and by frequent contact with his limited environment, the infant has begun to recognise what is familiar in his daily round; and the psychological mood, "at home," becomes an important factor in his happiness. As an outcome of this power, about the third or fourth month, he develops a fear of strangers. Of course, children vary much in this respect, and while one infant, which has been kept strictly secluded, will be alarmed at the sight of a new face, another, trained to variety in his surroundings, will regard strangers with complacency. Some infants are peculiarly quick in detecting differences. Thus, Baldwin's infant, when only two months old, was able to distinguish, in the dark, between the touch of his mother and his nurse. ${ }^{1}$ In cases in which it has been necessary to wean an infant, it is not uncommon to meet with well-marked aversion from the new food, though in consistency, colour, sweetness, and temperature it resembles human milk. ${ }^{2}$

1 Mental Development in the Child and the Race.

${ }^{2}$ Sour milk is customarily given by Kaffir women to their infants when about three weeks old, and this change of food is, as a rule, so strongly resented, that a mother requires to hold her baby's nose until the infant is compelled to open his mouth to breathe, when the curdled liquid is poured down his throat (Savage Childhood, Kidd, 1906, p. 38). 
The Fifth, Sixth, and Seventh Months.-The advances already made have been so considerable, that the differences between an infant at the beginning of the fifth week and at the end of the sixteenth week are very wide. This progress continues throughout the next three months, and we shall observe during this period, not so much the rise of new powers, as the broadening of those already in existence. Up to about the fourth month the infant has been engaged in developing for the first time his movements, sensations, and emotions, as well as his powers of association, recognition, and memory. Throughout the fourth and fifth months these faculties continue to expand, and about the sixth month a new mental state appears, which is destined to be of the very first importance. Here for the first time we meet with the first vague apprehension of ideas of self.

Movements show an important advance, not only because they now tend to belong to the higher levels of muscular action, but because the lowest type gradually disappears. The random movements of arms and legs which used to be seen in the infant, both in his contented and his discontented moods, become less noticeable. Another gradual change that has been made is the specialisation of the arms, to the exclusion of the legs, as servitors to the intelligence. At first the infant makes no more use of his upper limbs than of his lower, but before long the possibilities of the hands begin to be understood, and at the period now under consideration, the infant, while never weary of grasping at any object that comes before his notice, makes no real use of his legs. Any article which has been grasped is, if movable, conveyed for critical examination of its taste and texture to his mouth ; for the best understood sensations are still those connected with the lips and tongue. Naturally, of all objects, the child's feet are most frequently before his eyes. The toes are a never-ending source of interest, for they can be sucked without difficulty, and their shape and consistency provide unlimited occupation for an enquiring mind. Failing these, the infant will readily allow his attention to pass to his fingers, and sometimes he may be seen to clutch one of his own hands and convey it to his mouth, ignorant of the fact that the hand could 
find its way unaided by its fellow. As time goes on, the fascination exercised by either fingers or toes increases to an absorbing degree, and the infant will lie for considerable periods contentedly investigating them. The cause of this special interest we shall consider later.

By frequent repetition, grasping movements are executed with less uncertainty, and the child no longer makes a series of unsuccessful efforts before attaining his end. The muscular sense is thus shown to be undergoing development, and a harmonious working arrangement becomes established between sensations of sight and co-ordination of muscles of the upper limbs. This implies a knowledge both of distance and of relative position in space, and these two factors make it possible for the child not only to despatch, as it were, the arm on its errand, but to contract the muscles working the fingers at the precise moment when the hand comes within grasping distance of the object. This power of accurate localisation is cultivated only by long practice, and its perfection depends on three conditions: (I) the ability to refer to its source of origin a stimulus falling on corresponding points of the retinæ; (2) the frequent comparison by means of visual sensations between the successive positions in space occupied by the hand that has set out to grasp and the stationary positions of neighbouring objects; and (3) the knowledge of the position and attitude of the limbs. The last named is derived from muscle and joint sensations, and is the so-called muscle-sense.

About this time we notice another advance in instinctive muscular action, allied to the foregoing, and rivalling it in importance. The infant is no longer satisfied merely to balance his head, but attempts the more complicated co-ordination of supporting head and trunk in a sitting posture. At first the attitude is maintained precariously, and the child is apt to subside. Later he can sit alone, provided he keeps to a vertical position, but if he leans in any direction he is liable to overbalance. The indomitable perseverance that characterises this and every other stage in an infant's progress brings its desired reward, and by about the sixth month the child should be able to sit securely, even when he attempts such awkward movements 
as twisting his foot to his mouth, or bending forward to seize an object almost beyond his reach.

Reference has already been made to the improved interpretation of visual stimuli, and though corresponding advances take place in hearing and in touch, by means of which new ground is opened in the child's mind and his limited store of ideas augmented, the immediate results are less easily recognised. New experiences of every kind are eagerly welcomed, provided they are simple enough to be understood, and therefore do not cause fear or confusion. At the same time, the resulting feelings come to be more clearly expressed, though sometimes we can only gather evidence of the child's pleasureable state of mind from the fact that he will contentedly repeat an experience over and over again. It is worthy of note that auditory sensations are highly appreciated. A table will be repeatedly struck with a spoon, a bunch of keys will be shaken for minutes together, or, better still, a newspaper will be crumpled to elicit its crisp rattle. The latter action appears to be curiously fascinating, and more than one observer has noted the pleasure it gave his infant. Mere placid content is not the only demonstration of pleasure, and with increasing frequency, facial, vocal, and gesticulatory expressions of feeling are used. Laughter is associated with the keener forms of enjoymentfor example, the vigorous amusement of ride-a-cock-horsewhile vocal expression, though still far removed from speech, becomes sufficiently developed to convey distinctions of feelings. Special sounds are more definitely associated than before with certain feelings, such as hunger, disappointment, and pleasure. The last named especially acquires a definite vocal expression, and an infant engaged in some interesting occupation will often "talk to himself" in an inarticulate babble so long as he is left undisturbed. About the same time the characteristic sound known as "crowing" is heard. Then we observe exclusively facial expressions. The child stares at strange objects, and depresses the angles of his mouth as a sign of discomfort. This has been noticed in the fifth month. Gesticulation appears as the use of the arms becomes better understood. A pleasurable experience that provokes laughter may further 
cause the child to beat his arms either against his sides or in front over his diminutive lap. Desire he expresses by stretching out his arms.

The zest now displayed by the infant in his surroundings implies not only a multiplication of ideas, but an increased power of association of those ideas. Recognition becomes more certain, and memory gradually assumes importance, though the earlier memory-images are too short-lived to be of much service, and the infant has to learn anew to-day what he seemed to have fathomed yesterday. This feebleness or discontinuity of memory is dependent on the weakness of the associations established between ideas, and is one of the main differences between the young and the adult mind. The first proofs of memory are often provided by imitative muscular action, which is, of course, only possible when the memory of the original action survives. These acts, however, are not often attempted at the present stage.

The most interesting development about the sixth month is the dawning of the consciousness of self. This consciousness is impossible until experience has reached a relatively advanced stage, and reasoning has made its appearance. In the first three or four months the infant has shown no capacity for arranging his experiences under the two heads of subjective and objective. He holds all objects at the same impersonal value, and whether he is regarding his own body and limbs or his rattle, the "I-idea" is dormant. The earliest glimmerings of the consciousness of self appear in connection with the primitive sensations of hunger and thirst, though these must be experienced very many times before they become associated even in the vaguest manner with the infant's own individuality. Quicker progress is made by the help of the limbs, and these owe their special influence to various circumstances. The first, which has already been mentioned, is their constant and unavoidable presence in the immediate neighbourhood of the child. Next, they are endowed with the power of motion, and moving objects rarely fail to arrest a child's attention. Lastly, though of chief importance, the limbs possess a quality which all extrinsic objects lack; they are sensitive. An infant that is 
handling, for example, a wooden brick derives from his fingers sensations of touch, of temperature, and perhaps of weight, but when he drops the piece of wood and grasps his toes, stimuli now stream up to his brain, not only from the hand that grasps, but also from the toes grasped. The infant is not able at first to appreciate this difference, and is longer still in associating this peculiar quality with those objects which alone possess it, and in marking them off in his own consciousness from those that lack it. But even before this understanding is reached, the child will prefer to play with his toes rather than with a piece of wood. He derives more sensations and therefore more pleasure from the former. Gradually there is borne in upon his mind the fact that a difference exists in the objects touched, and the earliest appreciations of this fact are associated with wonder. Thus an infant grasping hither and thither, happens by chance to seize one hand with the other. $\mathrm{He}$ is plainly astonished. He becomes quite still and steadily stares at his interlocked fingers. Then slowly opening the enveloping hand, he supports in its palm the fingers of the other. Again he stares mystified. He begins to feel the fingers of the passive hand, bending them open and shutting them again. This investigation is continued, until finally the hands move apart, and the child's attention wanders to other things. Preyer noticed his infant in his fifth month studying his fingers. A few weeks later, he obtained possession of a glove, and showed unmistakably that he was comparing this strange hand with his own more familiar variety.

The sense of touch is therefore of the very greatest importance in laying the foundations of the consciousness of self. At a later date other factors come into operation. These are pain, the knowledge that changes can be effected on objects by efforts on the part of the child, the constant use by adults of a special name for the child, and so on. But though some of these may arise in the period under present discussion, their influence is not fully established till later, and therefore their consideration will be deferred. Mention should, however, be made of the experiments carried out by various psychologists to test the ability of an infant to distinguish between himself and his 
image in a looking-glass. It is well known that in the first three or four months of life an infant betrays no sign of recognition when he is held before a mirror; indeed, he appears not even to see his reflection. Later, the image will be seen, and will be regarded with attention, and later still, it may provoke smiles of pleasure. At this stage the child does not necessarily interpret it as an image, still less as his own image; his knowledge of self is too rudimentary to permit of this deduction, for though his limbs and even his trunk might be endowed with some degree of personality, the infant cannot possibly recognise his head, his face, or his neck. When the image of another, say his father, is observed, the recognition is less difficult, since the child is familiar with the facial expression of the parent, and is therefore able to appreciate the resemblance between him and his image. Confusion, however, is likely to result at first, when the infant thinks he sees the same individual in two places, and an attempt may be made to solve the difficulty by openly comparing the original with the counterfeit. In spite of this most intelligent act, some perplexity may remain until the child is able to assist himself by his sense of touch, or by hearing that the familiar voice comes only from the one direction.

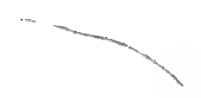




\section{CHAPTER IV}

THE PSYCHOLOGY OF CHILDREN (concluded)

The Eighth, Ninth, And Tenth Months-Instinctive Movements; The Static Sense; Judgments of Distance ; Capacities of a Child at Nine Months; Conscious Imitation; Attempts to Stand; Understanding of Words. THE END OF THE FIRST YEAR-Locomotion; Psychological Importance of Walking; Other Co-ordinated Movements ; Curiosity; Stages in the Development of Speech ; Intellect ; Judgments ; Reasoning; Appreciations of Causality; Experimental Investigations by Infants; Repetition of Experiments ; Consciousness of Self. THE SECOND YEAR-Locomotion; Climbing Stairs; Use of Joints; Speech; Monosyllabic Words; Mistakes in Use of Words; Consciousness of Self still Imperfect; The I-feeling; Intellectual Advances; Limited Experience responsible for Erroneous Judgments; Obedience; Imagination. THE THIRD YEAR AND LATERComplex Muscular Movements ; Skipping ; Jumping ; Colour Sense ; Emotions more Complex; Timidity; Sympathy; Affection; Ideas of Altruism; Use of the First Personal Pronoun ; Imagination; Intellect.

Eighth, Ninth, and Tenth Months.-From the end of the seventh month the infant continues to perfect his sensori-motor mechanism, without, however, attaining any new power of great importance, until the end of the tenth month, when the first attempts at walking are usually made. If, therefore, the progress in this period - the eighth, ninth, and tenth monthsis considered in somewhat less detail than in the preceding three months, this brevity must not be held to imply that we are now dealing with an interval of comparative inactivity, but rather that the infant, during this time, is maturing his abilities, and that the results of this preparation will be shown only at a later stage. 
During these months the instinctive movements of the arm become much brisker and better controlled. The static sense ${ }^{1}$ and the power of forming judgments of distance are now sufficiently developed to allow an accurate use of the hands in grasping. Additional pleasure, interest, and astonishment are evinced in the efforts, and the child shows an unquenchable eagerness to seize every article within notice. The general state of intelligence at this period will perhaps be best appreciated by an example.

An infant girl of nine months was allowed to sit upright on her mother's knees, and after a short interval, was offered the cap of a fountain-pen, made partly of bright metal and partly of polished vulcanite. It was immediately grasped with one hand and visually and tactually examined, both hands being employed together. It was then taken from the child and held within easy reach above her and to her left. Her eyes had continuously followed the object, and she quickly regained possession of it with her left hand. In whatever position in the field of vision the cap was held she invariably seized it with the nearest hand. There was no preference for the use of either right arm or left; both were employed with equal success and were able to locate the article unerringly.

The tests were then repeated by holding the cap sometimes within easy grasping distance, sometimes a little beyond arm's length, at other times farther off still. The infant was able to judge to a nicety whether or not she would be able to grasp it by a movement of the arms alone. When this was not possible she bent her body forward or laterally, according to necessity, at the same moment that her arm began to move, and did not first make her attempt with the arm alone. Clearly, therefore, a visual judgment of distance was formed before each effort. When the cap was too far away to be reached by the combined arm and body movement, the difficulty appeared to be at once recognised; for the child first paused to regard the object, and then strained forward to her utmost towards it. At whatever distance the cap happened to be placed, the hand was advanced

1 The sense by which we know our position in space relative to the outside world. 
three-quarters open, with the thumb held ready for opposition to the fingers, and this method of grasping was invariably employed.

Next, the cap was held within easy distance in the open hand, and the child allowed to take it away some half-dozen times in succession. On the next attempt, the observer's fingers suddenly closed, so that the cap disappeared from sight. A perplexed look quickly crossed the infant's face, followed by a more prolonged expression of wonder with eyes wide open. She irresolutely touched the hand, but made no effort to open its fingers; then looked to right and left, obviously searching for the missing article. In this endeavour she happened to catch sight of the corner of an adjacent table, towards which she immediately leant to pat its surface, and the cap was at once forgotten. A little later it was again given to her, and she was permitted to retain it, to her evident enjoyment. As she handied it, she uttered sounds of satisfaction, in which an occasional consonant could be detected. During this interval, changes of expression played about her face, her eye-brows especially beginning to twitch, while her lips were parted She then carried the cap to her mouth for investigation, but experienced some little difficulty in finding her lips, and by mistake thrust the projecting metal end against her cheek and nose, and once against her eye. Finally, she removed it from her mouth, but by accident dropped it on the floor. She was quite unconcerned at the loss, made no effort to trace the article, and after a few moments began to dance herself up and down, beating her lap with her hands, laughing, and crowing loudly.

This account of the incidents of a couple of minutes will convey an impression of the powers of movement, sensation, expression, speech, attention, and intelligence of an infant of some nine or ten months. One or two further details will be required to complete the picture. About this period efforts at conscious imitation begin to develop. Preyer thought he saw an imitative act (pursing the lips) in his child when only fifteen weeks old, and he noted further progress in the seventh month. It is probable, however, that few infants are capable of conscious 
imitation until about the ninth month. Romanes's infant son, ${ }^{1}$ at eight-and-a-half months, had been taught to shake his head at "no," to nod it at "yes," and to wave his hand for "ta-ta." The last-named action is usually one of the earliest instinctive movements to be learnt; but children may, of course, be able to perform an action before they can appreciate its significance. Darwin ascribed the emotional expressions of young children to conscious imitation, yet the earliest of these can hardly arise in this way. Open-eyed wonder is among the first expressions of infancy; yet the child of educated parents can but rarely have an opportunity of observing this facial change in his parents.

About this period the highly elaborate mechanism required in walking begins to pass under control, and the infant is no longer satisfied with sitting, but tries to stand or crawl. He has practised co-ordination only with his arms, and has but little power over his legs. Their movements are crudely performed, but this is due rather to want of practice than to weakness, for the leg-muscles have become sturdily developed by frequent kicking movements. In the first unaided attempts at standing, the arms help the body by dragging it into an erect posture. The further development passes through regular stages, the earlier of which may be briefly mentioned. The legs first learn to support the weight of the trunk, and later the burden is temporarily transmitted through one limb at a time. Then the child, having gained some confidence, begins to kick out with one or other foot, always, however, maintaining his security by the help of his arms, as the power of equilibrium is still immature. If at about this stage the infant is assisted in his attempts at locomotion, his leg action will be as uncertain and irrogular as that of a man with ataxy. The movements will be jerky, the feet will be raised too high, and perhaps brought down on the ground at the same spot from which they have just been lifted. The toes will trip against the floor, while the necessity of moving the limbs alternately will not always be realised. $m$ infant can stand alone only after considerable practice, and, for a longer time

1 Mental Evolution in Man, 1888. 
still, he will show much hesitation in attempting to walk unaided.

The power of articulate speech makes but little advance in this period, and the child may fail to repeat monosyllables, though he may nevertheless possess some understanding of the significance not only of words but of sentences. An increased flexibility of voice usually occurs about this time, and one or two additional consonants may be detected in the infant's prattle. The date when the power of speech is first attained varies very widely in different children. Romanes recorded a vocabulary of four words in one of his children when eight-and-a-half months old, but Preyer's infant was more than a year before he first attempted to repeat a word.

\section{The END of THE First YeAR}

The Eleventh, Twelfth, Thirteenth, and Fourteenth Months.Passing now to the next series of developments, we may conveniently select for description a child that has reached his first birthday. We must remember that different individuals progress at different rates, and so we shall include in our account observations made from the end of the tenth month to the end of the fourteenth month. ${ }^{1}$

During this period the child devotes much attention to improving his locomotive powers, and, when incapable of success with his legs alone, goes on all-fours. The psychological importance of the ability to walk cannot be overestimated. The child's principal interest is no longer restricted to the immediate neighbourhood in which he sits, but henceforth he is able to concern himself with a wider area, and, in nursery, garden, or field, discovers a thousand and one novel delights that urge forward his mental development at a great speed. New experiences are of frequent occurrence, and the eager intelligence of the child absorbs them like a sponge in water.

1 For an interestin cord of the powers of an individual child of twelve months, the reader should refer to Major's First Steps in Mental Growth, p. 353, where there is a summary of "What the Baby did in Forty-five Minutes," on his first birthday. 
Among the more complicated movements that may be observed about this time may be mentioned those of throwing and playing with a ball, building with wooden bricks, feeding from a spoon, and drinking from a cup. Imitative movements are executed more readily, and with them the will is developed.

The emotions and their expression now show further advance, and the new conditions of the child's life provide him with many occasions for astonishment and pleasure. In the keenness of his enquiry into each and every matter that comes before his notice, he often loses the timidity and bashfulness that appeared when first he was learning to distinguish the familiar from the strange. For some months to come, until the consciousness of self has made its full appearance, he is unlikely to allow any diffidence to interfere with the satisfaction of his curiosity.

By the end of the first year the faculty of speech begins to take those strides forward that will soon place it for ever among the foremost mental attributes. We may therefore consider at this stage, and in some little detail, the earlier steps in speaking.

The primary origin of the faculty of speech is customarily traced to the reflex crying of the new-born infant, and its earlier manifestations are continued through the inarticulate noises, such as whimpering, crowing, and grunting, which become differentiated to express the elementary feelings of comfort and discomfort. In the use of these sounds, which have been grouped together as "prelinguistic babblings," the infant employs, by chance, many of the vowels of adult speech, as well as certain of its consonants-especially labials-and probably by the end of the first year the majority of the sounds that make up language have been formed.

The next stage is the establishment of a mental association between a sound heard and a particular object or experience. An infant may thus come to understand the meanings of words; or even of sentences, at a time when he is incapable of repeating even one monosyllable. Thus, at the words, "Where is Nanny?" a child may be able to point to his nurse, even though he 
cannot himself utter any of these three words. It is hardly necessary to state that when interpreting a sentence such as this, he does not regard the sounds as a series of words, but rather accepts them as an undivided whole-" whereisnanny." This power of understanding speech may antedate the use of words by many weeks, but by the end of their first year the majority of infants have made obvious attempts to speak. The day when the first word is correctly uttered is an important one, and the act proves the existence of an intelligence that is already complicated. Not only must the elaborate neuromuscular mechanism involved in articulation be well controlled, but the child must be proficient in ideation, association, judgment, and (since speech is imitative) in memory.

Different words come into use along different psychological channels. Thus the child may have an idea (dog), and later may learn to imitate a certain sound (bow-bow), but only subsequently associates the idea with the sound. Or he may learn a sound (moo), and later form the correct idea with which to associate it (cow). Or he may simultaneously acquire the idea (watch) and the sound (tick-tick). When the idea and the word have become associated, each is able to call up the other, so that the idea of a thing prompts the name of it, or a sound heard calls up the idea or experience. One or two characteristics of these early linguistic imitations require mention at this stage. Almost all children are ready to coin words of their own, and their memories are apt to be very tenacious of these inventions, in spite of all the efforts of parents to substitute the proper words. Thus one infant employed the term "yay-yay" to express "milk," and persisted in the use of this word for many months. Another expressed the qualities of nastiness, coldness, and dampness by the sound "ga-ga." Sometimes children show considerable facility in this habit, and instances have been recorded where imitative speech has been completely excluded by language invented by the child himself. ${ }^{1}$ Another and not unnatural defect in early speaking, is the imperfect rendering of words imitated. The child is not.yet sufficient master either of

1 Some interesting examples were collected by Romanes in his Mental Evolution in Man. 
his hearing or of his lips to enunciate any but the simpler combinations of sound. Thus "policeman " became "pummum," and "Bertie" "Buggie." When a new word has been learnt, a child will take obvious pleasure in its use, and, if left to himself, may be heard contentedly repeating it over and over again.

The above stages in speaking are reached approximately at the end of the first year. Further developments will be dealt with below, but we must now turn to the child's intellectual condition on his first birthday.

By this time ideas are formed and judgments are made with comparative ease. The association of ideas has become stronger, and memory has developed until it can re-awaken ideas after intervals of many days. The child has now become sufficiently familiar with the commoner properties of objects to be able to detect changes produced in them by altered conditions, and to look for the reason of those changes. Thus Preyer's infant, on his 319th day (45th week), striking a plate with a spoon, noticed and ascertained the cause of a difference in sound when the vibrations of the plate were damped by his hand. $\mathrm{Kidd}^{1}$ records an incident in which a Kaffir baby of one year touched by chance the hot lid of a vessel that stood in a native hut. The resulting sensation perplexed the child beyond understanding, and he stretched out his hand a second time to the pot, again burning his fingers and being filled with surprise. Only after a third contact did the child show by his crying that he had no further doubts as to the connection between the pain in his hand and the lid of the pot.

This appreciation of causality leads the child to attempt to produce changes (especially of position) in his surroundings, and he is no longer satisfied merely with investigating the shape, smoothness, texture, and other properties of articles in his possession. His toes and fingers begin to lose some of their interest, seeing that but little more can be learnt from them, and the child, now able to move from place to place, begins that desirable and highly educative practice which, unfortunately, is still known to many parents as "getting into mischief."

\section{Savage Childhood.}


Whether the experiment of the moment is the tearing up of a letter that the wind has brought to the floor, the uprooting of a plant in the garden, or the insertion of a finger into a fellowinfant's eye, the actuating motive is the same-the observation of the effects of voluntary action. Of these effects, the one that perplexes the infant's mind most is alteration in position. An article is seen in a certain place; it is then made the object of voluntary action. It is now seen in another place, and its original site is observed to be vacant. Very many such experiments have to be made before a child understands the meaning of this change. Major, in his account of how a oneyear-old baby spent forty-five minutes, tells us that the child while playing on the floor, picked up a walnut with his left hand. Slowly and with great care he transferred the nut to the other hand, and then steadily regarded the empty left. Nine times in succession did the child repeat this action before his interest passed to other things. ${ }^{1}$ Major expresses himself as at a loss to explain this strange proceeding, but remembering the facts already set out above, we must conclude that the child was experimenting along the lines just described. So little did he understand the connection between voluntary action and change of position, that the sceptical and persevering mind of this truly scientific enquirer refused to be satisfied until the experiment had been repeated nine times with unvarying results.

Incidentally, it may be remarked that infants will repeat their experiences without wearying over and over again. One observer records how his child took off and replaced the lid of a can seventy-nine times in succession.

Finally, we must note at the end of the first year the further developments of the consciousness of self. The infant is now beginning to clarify his ideas in this connection, and, though he has not yet awakened to the fact that his bodily parts are essentially different from all the other objects of his attention, he nevertheless shows unmistakably that he realises that there is a problem to solve, and he is constantly attempting to find the answer. Preyer, who gave special attention to this point,

1 First Steps in Mental Growth, p. 353. 
narrates how his infant was seen to hit alternately a table and his own head, obviously attempting to discriminate between the sensations that resulted. He struck his body and attentively regarded his hand. He compared his own fingers with those of others. On one occasion, while holding to the side of his cot, he bit his bare arm so vigorously that he cried out with pain. All these experiments were made between the tenth and the fourteenth months. Allowed to look at his image in a mirror, he slipped his hand behind the glass to touch what was evidently regarded as real. The same action followed when he was shown a photograph of himself, yet when looking at the reflection of his mother, he had no difficulty in distinguishing between the image and the parent.

The chief factors now at work in building up knowledge of self are: the sense of pain, which is ever ready to mark off the subjective from the objective; the perception that changes can be effected by the child's own activity; and lastly, the information obtained by comparing others with self. All these influences become increasingly important in the second year, and will be considered below.

\section{THE SECOND YeAR}

In dealing with the child who has completed his period of infancy and is already well embarked on his second year, we shall no longer need to trace in detail the further progress of the more elementary psychological phenomena, such as muscular co-ordination and sensations. These continue to develop along the same lines as hitherto, but the more noticeable features of the second year will be found in the increasing complexity of the mind. The faculties which require special consideration at this stage are speech (including the growth of the vocabulary and the formation of simple sentences), consciousness of self, intellectual powers, imagination, and ideas of obedience. Before passing to these, a few remarks must be made on locomotion.

The infant who a few weeks back was just learning to stand is now able to move without difficulty from place to place. He has probably advanced this accomplishment to the stage of 
running, and can eren alter the direction of his course without disaster. In time he learns the mechanical advantage of bending forward as he runs, and finally, locomotion becomes sufficiently mechanical to allow the child to relax his attention from the effort, so that he can look about as he goes, and take notice of his surroundings. Flights of stairs that were climbed at first on all-fours, then with the help of the bannisters, are now mounted, and later descended without assistance.

Even at this stage, when both arms and legs have become fairly well trained in their respective functions, it can be remarked in all children how little able they are to take advantage of the jointed actions of their limbs. A young child, as he runs, swings his arms more or less stiffly from the shoulders, not yet having learnt the value of bending the elbows and semi-pronating the forearms as in doubling the arms. If a ball is gently thrown towards him to be kicked back again, his leg will move awkwardly about the hip-joint, and action at the knee and ankle will rarely be attempted. In other words, the muscle-sense, which is derived largely from the contractions and relaxations of groups of muscles, and from sensations of strain and pressure about the joints, is in an elementary condition.

The child's vocabulary, acquired entirely by imitation of sounds heard, now begins to attain respectable proportions. Monosyllables are the first to be mastered, and the earliest disyllables are those in which, as in "mama," "papa," the second half of the word is merely a repetition of the first. Mistakes are not uncommon at these stages. A child may, owing to a false association, persistently attach the wrong word to an object, yet the word in its new meaning will be used with logical correctness. It is a very common error for a word to be used to express different objects, which, to the adult understanding, have but little in common. A child of eighteen months, who was familiar with the appearance of a dog, saw a couple of black lambs among a group of white "lambies," and hailed them with "Eh, doggie, doggie." 1 The broad points of resemblance between the $\mathrm{dog}$ and the lambs were at once 
seized on, while the differences in detail passed unnoticed. This peculiarity of the child-mind-a quick apprehension of broad similarities and a deficient appreciation of slighter dissimilarities-is, of course, an expression of their undeveloped powers of observation, itself the result of a necessarily limited experience. In many instances a word is used erroneously from its association, not with the object but with some special quality of the object. Thus a small boy called a feather that was blown away for his amusement, "bow-way." Later, he applied the same term to a pigeon that flew off at his approach. ${ }^{1}$ Here the name was mentally associated rather with the movement of the feather than with its physical properties.

When once a word has been misapplied in this manner, it may, by a process of extension, finally come to be used for the most unlike objects. A child, watching some ducks on a pond, learnt the word, "quack." The name was then applied not only to all birds, but to all insects; to water, and to all other liquids. Later, the child, having seen a French sou on which was stamped the figure of an eagle, extended the term to the coin; so that in the end the word "quack" was applied impartially to a fly, a glass of wine, and a piece of money."

In spite of mistakes such as these, a child in his second year stores up an ever-widening vocabulary, and soon attempts to piece together short sentences. These are first learnt about the second half of the second year, and consist merely of a couple of words- "baby good," "kiss brother." If a negation is attempted, it is usually expressed by the word " no," and this may be either prefixed or suffixed to the other word of the sentence, so that "no bed" or "bed no" would equally express "I do not wish to go to bed." Of onomatopœic words, which often figure prominently in a child's talk, it can hardly be doubted that they are not onomatopœic so far as the child's first use of them is concerned, for they are almost invariably learnt from adults. In this connection it may be noted that the same animal sound will be expressed very differently by children of different nationalities. Thus the "cock-a-doodle-

1 Major, op. cit.

${ }^{2}$ Romanes, Mental Evolution in Man. 
doo" of this country becomes the "kikeriki" of a German child and the "coq-coq" of a French.

The consciousness of self continues to develop, though it is still far from being firmly established. The child now clearly recognises his ability to effect changes in objects, but may nevertheless show, by such actions as biting his skin, and offering food to his feet, that further experience is required before he can realise his proper relation to his surroundings. In his study of other individuals, he has passed the stage of regarding them merely as objects of interest, and begins to hold them as akin to himself. This conception is greatly helped by the growing use of speech, and the I-idea is fostered by the child's constant experience of hearing himself addressed by name. How imperfect the feeling still remains is shown, however, by the invariable use, in speaking, of the third person"baby jump," not "me jump." At this period speech comes to the foreground in building up ideas of self. Romanes, ${ }^{1}$ indeed, maintained that up to the time when a child begins to speak, he is without consciousness of self ; but Preyer ${ }^{2}$ has shown that the I-feeling is present before any words are learnt, though speech undoubtedly plays a very important part in making it more exact.

The intellectual advances in the second year are very considerable, and receive their greatest help from the increased powers of speaking and of understanding speech. The limitations, however, are still narrow, and are often revealed in acts which are the result of imperfect experience. Thus a child who possessed all the reasoning power and memory necessary to play hide-and-seek attempted to catch hold of tobacco-smoke. At seventeen months he had the intelligence to obtain the assistance of a bag on which to stand in order to reach an object beyond his grasp, yet a month later continued to use a garden watering-pot even after it had become empty. ${ }^{3}$ In contrast to acts such as these, children begin about this age to show that rigid logic in the application of their knowledge

1 Op. cit., pp. 202, 211.

2 Mental Development in the Child.

${ }^{3}$ Development of the Intellect, Preyer. 
which later prompts these unanswerable questions with which every parent is familiar. Erroneous judgments are frequently made either from insufficient data or from the habit of generalising from the particular. This latter practice is one very commonly adopted by children, who experience no difficulty in mentally transferring qualities from one object to another.

Two new psychological developments of this age must be briefly mentioned. Obedience is one of the first moral qualities to make its appearance, and usually begins with a knowledge on the part of the child that he must not perform some particular action. Its further growth depends beyond everything on those in authority, and if they are slack, inconsistent, or oversympathetic, the child quickly learns to assert his independence.

Imagination, a faculty which is destined to play so large a part in the child's later life, is founded on memory-images, and is the freer because the child is not hampered in his mental excursions by the weight of much experience. He can therefore readily devise situations and bestow qualities which a wider knowledge would show to be absurd. Specially frequent is the habit of conferring human attributes on animals or even on inanimate objects. A boy of two, hearing a bee buzzing against a window-pane, remarked, "Mamma, bumble-bee in a window says he wants a yump of sugar." Then remembering the effect on little boys of eating too much sugar, he shook his head sternly at the insect, and added, "Soon make you heatspots, bumble-bee." 1 Another boy, of similar age, was sitting on the floor in a bad temper when the sun suddenly burst into the room. This publicity was too much for the disconsolate child, who said, captiously, "Sun not look at Henny." The sun, however, went on shining, and the boy again addressed it, this time pleadingly, "Please, Sun, not look at poor Henny." 2 Examples such as these are not uncommon in early childhood, but become less frequent as the relationships and distinctions in the world around are learnt.

$$
1 \text { Sully, op.cit. } 2 \text { ibit. }
$$




\section{Tile Third Year and Later}

By the end of the second year the mind has acquired in an elementary form most of its principal faculties, and henceforth its progress will consist rather in perfecting existing functions than in establishing new. Muscular movements become elaborate. Jumping, skipping, and so on, are successfully attempted, and the hands gradually acquire the power of carrying out such complex acts as threading beads, or plaiting straws. In connection with the senses, the most noticeable change is, perhaps, the awakening of the colour sense. This is one of the latest of the sensory developments, and, according to Miss Shinn, its existence has never been definitely proved before the second year. It is not till a year later that the appreciation of colour begins to assume any prominent shape in the child's mind.

As the emotions continue to increase in complexity, timidity and fear begin their strong influences on the child's little world. They arise in the first place, as has been pointed out, from the infant's dislike of strangeness or novelty, and they still result from the same causes. Not only may the child be afraid of new people or things, but he may be timid in the presence of objects such as animals, the actions of which he cannot anticipate or control. Children are often frightened if taken by surprise, and they are specially alarmed to see any object, such as a clockwork toy, reveal some unsuspected quality such as movement. Thus Sully records the incident of a little girl who, at the bedside of her mother, pulled out of an eiderdown quilt a feather, which began to float across the room. The child's fright was great when she saw an inanimate object suddenly developing the power of motion. ${ }^{1}$

1 It is interesting to compare this incident with one recorded of a Skye terrier by Romanes. The dog in question was fond of playing with dry bones, tossing them in the air and worrying them under pretence that they were alive. On one occasion Romanes tied a long thread to a bone lying stationary on the ground, and at the moment when the dog was running towards it he gently pulled the thread and the bone began to move. Instantly the dog's whole demeanour changed. The bone which he had merely pretended was alive now seemed in reality to be so, and his astonishment knew no bounds. Finally, in dread of the still receding object, he ran away and hid himself. (Mental Evolution in Animals, p. 156.) 
One or two further points may be mentioned in connection with the emotions. An outstanding feature of childish emotions is, that though they are overpowering in their intensity, they are always short-lived, and prolonged emotional states, such as depression, are very rare. Tears which, up to about the end of the second year, used to be shed from anger or annoyance, now begin to flow at the call of grief. About the same time sympathy and affection grow deeper, and the child is easily moved by them. Girls as well as boys at this age have been spoken of as shockingly callous, but though they may sometimes show a complete absence of feeling on occasions such as bereavement, their indifference is more justly ascribed to ignorance. They have, on the contrary, an all-comprehensive sympathy, which embraces the inanimate world as warmly as the living; and the experience is not unique of the child who felt so genuinely sorry for the stones in the road with their monotonous existence, that she would carry them in her basket, to set them down where they might enjoy a fresh outlook.

Out of the primitive emotions of this age the future morality of the child will grow, and though for a long time the young are concerned only with their own wants and pleasures, this egoism gradually melts before other influences. As the relation of self to others is recognised, ideas of altruism show themselves. These cannot exist until consciousness of self has been established, and the final stages of the latter are developed, as we have seen, by means of speech. /The day when a child first uses the personal pronoun "me," is an important milestone in his life. It usually occurs in the third year, and is soon followed by the more exact substitution of "I " for "me." Inflection of verbs to distinguish persons is a further aid, and finally, the child comes to appreciate clearly his selfness. So great is the interest he takes in his newly found individuality, that selfness cannot avoid becoming selfishness, and it will now be many years before the child succeeds in regarding the world except from a standpoint which is at root selfish. From the day when in endeavouring to stand without assistance he showed his earliest ambition, his attitude may be summed up 
in the words "me do it," and not until some considerably later period do altruistic motives influence his life.

Imagination begins to acquire a fuller sway, and finds outlet in every direction. Not only will it guide children at their play, but even in their more serious affairs it may occupy a prominent place. As a result of this, children are often led to make assertions which are the products of their imaginations, and which have no foundation in fact. Adults have difficulty in appreciating this distinction, and are sometimes apt to assume that a child has been lying, when he has been innocent of any such intention.

The intellectual development proceeds along the same lines as those already traced, and does not require to be treated in further detail. Month by month the powers of observation become more acute, and the understanding soon reaches a level where it deserves comparison with the adult mind. With increased manual dexterity a liking arises for simple industrial or mechanical exercises. Some skill in drawing is a not infrequent accomplishment, and by the time the finer movements of the fingers come under control, the power of writing can be acquired. The child has now placed himself beneath the shadow of our scholastic system, and henceforth his development will continue in accordance with the rules of the classroom, and not with the laws of nature. This new influence will be considered in a later chapter on the training of children. 


\section{CHAPTER V}

\section{HYGIENE OF SCHOOLS AND OF SCHOOL LIFE}

INTRODUCTION-Importance of School Hygiene; Parents and School Hygiene; Hygiene of Boarding Schools. HYGlene of ScHoolsBoarding-Schools : Site, etc. ; Dormitories ; Internal Arrangements of Dormitories ; Their Cubic Capacity ; Their Furniture ; School WaterSupply ; Milk-Supply ; Laundry-Day Schools : Site, etc. ; Classrooms ; Size ; Ventilation ; Artificial Heating; Natural and Artificial Illumination; Wall-colouring; Furniture; School Desks. Hygiene of ScHOOL L1FE-Food: Cintines Scolaires of Paris ; Food at BoardingSchools; Times and Numbers of Meals; Alcohol; Hours of Sleep ; Mental Fatigue; Physical Strain of Classroom Work ; Intervals for Exercise; Daily Programme of Work; Number of Working Hours; Home-lessons; Holidays; Relation between Work and Play; School Games ; Clothing of School Children.

\section{INTRODUCTION}

IN shaping a child's physical, mental, and moral natures, the school plays a part second only to that of the home. During the period devoted to scholastic training, the potentialities of the young, malleable and ductile at the outset, develop into the firm and deep-rooted qualities of character and temperament that remain unalterable throughout later life. This process is so readily modified by surrounding influences, that, if a child is to be turned out with his powers cultivated to the best advantage, no detail of school life is too insignificant for our study. Yet in spite of the recent growth of public interest in the welfare of the young, a widespread indifference to the scholastic environment of their children still survives among many parents, even of the upper and middle-classes. Boys and girls who have been brought up wisely and with every home 
care are sent away, with scarcely an enquiry on the part of the parents, to schools where the living arrangements, feeding, or drainage are of a kind that would not be tolerated elsewhere.

Such unconcern is to be ascribed rather to ignorance than to apathy. The average parent has no special knowledge of schools, and he is acquainted only in a general way with the features in which they are most commonly deficient. Even should he be somewhat better informed, it is improbable that he would know what enquiries or investigations are necessary before entrusting his child to some particular school. His difficulties are the greater because he is tied by many considerations apart from those of health. Excluding financial reasons, which must in the majority of cases limit the choice, he is influenced by such matters as the social position of the school, the presence in it of one or two boys who will befriend his child, the headmaster's reputation, the probable future occupation of his boy. It is right that these points should be taken into account, but all of them together should not be allowed to outweigh the single question of health.

This opinion is only slowly diffusing itself through the country, and at present is rarely acted on except with regard to delicate children and those who come of a stock that makes possible the future development of tuberculous, mental, or other disease. In this connection parents are beginning to realise that preventive measures may ensure their offspring an escape from a family weakness, and they therefore decide on their children's scholastic life only in consultation with a medical adviser, who can point out what dangers are to be avoided, and what conditions are to be sought. A great responsibility is thus placed on a doctor in arranging the early life of a delicate child. He requires to investigate very carefully, first the family medical history, and then the exact condition of the child before him-his capacities and limitations, both physical and moral. His opinion will be based on this double foundation, and he has then to decide how far the wishes of the parents or their choice of a school is compatible with the child's best interests. Whether he is able to support the suggestions of the parents or finds it necessary to urge an alternative course, that medical 
adviser will do best for his patient who realises that the question of health must take precedence of all other considerations.

Unhygienic conditions prevail perhaps more frequently in boarding-schools than in day-schools, for the latter are usually more modern, and, lying in or near towns, can more readily be provided with good sanitation. Yet healthy surroundings are, if possible, even more important in boarding-schools, for the inmates are exposed both day and night to possibly harmful influences. It is well known that schools, even of the highest repute, leave much to be desired in this direction, and were it not that their social prestige and antiquity obscure the defective precautions taken to safeguard the health of their pupils, parents would be less eager to entrust their children to such surroundings. A leading authority on the hygienic conditions of our public schools has summed up the matter in the following words: "Nothing short of government inspection of our highgrade schools will even make them approximate to an ideal condition: and even this plan would be insufficient without an annual publication of all cases of infectious disease occurring at school." 1 The obstacles in the way of these remedies are not likely to be overcome except as the result of outside pressure, which must be exerted by those primarily interested-namely, the parents.

The present chapter will deal with schools from the point of view of hygiene, and will be divided into two parts-one treating of general sanitary questions affecting school buildings, the other of the hygiene of scholastic life, including such topics as work and fatigue, recreation, and diet. In the former part the features peculiar to boarding-schools will be first considered, then those peculiar to day-schools, and finally the details of classrooms generally.

\section{Hygiene of Schools}

Boarding-Schools.-As regards the situation of a school, the same features should be looked for as in selecting an ordinary dwelling, with this proviso, that school-children, being at an age

1 Health at School, Clement Dukes, M.D., 4th ed., 1905, p. 45 I. 
when their growth is rapid and their constitutions impressionable, are more liable than adults to suffer harm from unhealthy conditions, such as dampness or cold. The ideal position is one elevated on a hill above the neighbouring country, so as to ensure both freshness of air and dryness of soil. This last named is of very great importance, and the ground should be of a porous nature, so as to drain off all surplus rainfall without delay. Chalk, gravel, or sand possesses this quality, but clay, especially if low-lying, allows the passage of water only with difficulty, and makes an unsuitable soil.

A possible drawback to an elevated site is that during some of the winter months it may be exposed to cold, but this defect is less objectionable than those so often associated with a lowlying position, shut in from invigorating winds, and constantly kept damp by the subsoil flow of water from higher levels around. Where an otherwise desirable position is subject to sharp, biting winds, means can usually be found to obtain all necessary protection, either by seeking the shelter of ground rising up towards the north, or better by taking advantage of the cover of a wood or screen of trees. If the former defence is chosen so that the school lies not quite on the highest ground, and houses stand between it and the summit, the efficient drainage of the latter becomes a matter of importance, for any leakage into the soil may be a source of contamination within the school boundaries.

The wisdom of erecting the buildings to face towards the south is commonly insisted upon, but of greater importance is a sunny aspect for the rooms occupied by the boys themselves. A south frontage is obviously of but little moment if the classrooms or dormitories are placed in the rear of the building.

A boarding-school is customarily planned on a system of "houses," provided its size permits of this arrangement, and the scheme has advantages not only for reasons of discipline, administration, and esprit de corps, but also of facilitating medical measures intended to check the spread of infectious disease. These houses each contain their own dormitories and, in many cases, their own dining-rooms, though in some schools a preference is shown for a single common dining-hall. Of all the 
structural parts of a school, there is none of greater importance from the health point of view than the dormitories, in which every boy spends at least one-third of his time. Though used mainly at night, these sleeping quarters should be arranged, wherever possible, to receive a fair amount of sunshine, especially during the earlier part of the day. Not only will this secure for the inmates the beneficial effects of direct sunlight, but by warming the rooms, will appreciably affect their inside temperature throughout the winter. In the summer months, dormitories which are freely open to the afternoon sun are, by evening, apt to become unpleasantly close, and, as is well known, children are commonly very intolerant of hot, oppressive nights. The difficulty is to arrange for a maximum of comfort at all times of the year. In practice it is easier to warm a cold room than to cool a hot.

The internal arrangements of a dormitory can be made on one of two plans. The room can be divided into a double row of cubicles separated by a middle passage, or the beds can be left unscreened by any partitions or curtains. Each system has its advantages and its defects. On the one hand, it is right to afford each boy as much privacy as possible for his personal hygiene, so as to foster the development of that sense of selfrespect which is so essential to all. On the other hand, there are moral reasons which render facilities for seclusion from the eyes of the præfects or the more healthily-minded boys highly injudicious, especially in the case of older boys. There is no doubt, however, that the harm that may be wrought by the provision of cubicles is more disastrous than any that can arise under the more open system. An arrangement least likely to be abused is one in which each boy is allowed for his ablutions a small space or cabinet shut off from general view, while the beds themselves are arranged openly along the whole length of the dormitory. A further point that must be borne in mind is that ventilation-an item of the highest importance-can be made much more efficient where the room is not broken up by frequent partitions.

The size of a dormitory, as measured by the cubic space per bed, is not always that which modern hygiene demands. From 
800 to 1000 cub. $\mathrm{ft}$. is a desirable allowance for each sleeper, and this minimum would be obtained in a room $12 \mathrm{ft}$. high, by spacing the beds $3 \mathrm{ft}$. apart and leaving a passage $\mathrm{I} 2 \mathrm{ft}$. broad between the opposite rows, the beds themselves being of the usual measurements of $3 \mathrm{ft}$. by $6 \mathrm{ft}$. This allowance leaves but little margin for furniture, the volume of which must, of course, be subtracted in estimating the available air-space. Efficient means should be provided to renew the air throughout the night, and, during the day-when the room ought to be unoccupied-open windows and doors should keep the air fresh and sweet. The best type of window is one that runs up to the height of the ceiling, and consists above of a hopper head with glass cheeks and an ordinary sash-window below the transom. During the night the upper part can be kept open, and the side cheeks will divert the air from falling on the sleepers.

Considerations of space have in some schools led to the adoption of folding-beds which can be shut up during the day, and the room converted to other uses. Such economy is indefensible, for not only does it make impossible the proper airing of the beds, but it transgresses the essential rule that growing children must not be allowed to sleep in rooms which are occupied during the day-time. For this reason the practice of requiring the older boys to use their studies for sleeping is harmful, especially as these rooms are often all too small even for day use.

A dormitory should be furnished only with such articles as are essential to its function. No play-boxes or trunks should be permitted beneath the beds, which should themselves be as simple as possible. Provision for washing, though sometimes made in the room, is preferably arranged in an anti-chamber leading to the bath-rooms (hot, cold, and shower). A point to be remembered is the occasional need of closet accommodation during the night, and if the school lavatories are not close at hand, a single closet should be attached to the dormitory, the atmospheres of the two being on no account in communication.

The remaining items to be very briefly considered in special connection with boarding-schools are the water-supply, milksupply, and laundry. 
The vital importance of a pure water-supply is fortunately sufficiently widely recognised by parents at the present day to make them demand this elementary requisite. There can hardly be a more insidious danger in a school than drinkingwater of dubious quality, but town's-people, accustomed to accept the purity of their own supply as a matter of course, often fail to appreciate the difficulty of securing a pure delivery in an isolated country district, or the many possible sources of contamination. The matter is one that cannot be investigated without expert knowledge, and it therefore behoves parents, before entrusting a child to any school which is not supplied by a local water company, to obtain fully satisfactory evidence that there are no risks in this direction. A water-supply that is drawn from a shallow well should be regarded with special suspicion.

The sinister part played by milk in conveying disease is not yet clearly enough recognised by the laity for them to insist on reasonable precautions against contamination, and it is probably rare for parents personally to satisfy themselves as to the conditions and quality of the milk-supply of a school. Any anxiety they may have on this score is sometimes allayed by the advertisement of the school authorities that the milk is supplied from their own cows. The small value of this assurance is appreciated only by those who know that such private dairies escape inspection under the Dairies, Cowsheds, and Milkshops Order, and may therefore be no better than the average country cowshed.

Laundries, which by receiving linen from houses wherein infection has been or still exists, are liable to play their part in the dissemination of disease. This danger is therefore present in any arrangement that allows the school linen to come into contact with soiled linen from other sources. Freedom from this risk can be obtained only by providing a private laundry. Where a school is large enough to warrant this expense, the precaution is highly desirable, but the cost is usually not small, and at the present day it is generally possible, in most neighbourhoods, to find a laundry conducted on hygienic lines. 
Day-Schools.-The conditions governing the choice of site in a day-school differ only in the matter of elevation from those already outlined in the case of a boarding-school. An elevated site would often make the school difficult of access, and entail so much extra fatigue on the children coming and going, possibly twice a day, that a more convenient, if less elevated, position would be preferable. The site of an urban school is exposed to disadvantages from which the country school is free. The first difficulty is to find a plot of land which is not bounded by busy highroads of traffic, or situated near noisy centres, such as market-places, railway stations, and the like. The clatter of carts and waggons not only distracts the children's attention, but unnecessarily fatigues the teachers, who have to pitch their voices against the noises outside. These inconveniences only too often suggest, as their remedy, the closing of all windows, and the children are thus sealed up in a vitiated atmosphere. The immediate neighbourhood of the school should not include any offensive manufactories such as gas-works or brick-fields, while an abundant supply of daylight, uninterrupted by surrounding buildings or trees, is essential. In public elementary schools, a clear space of at least sixty feet is required between them and the nearest building.

Private day-schools, being usually adapted only for a small number of pupils, often possess structural arrangements which are no more ideal for education than are those of an ordinary dwelling-house. Rate-provided schools are built on wellrecognised models, and in accordance with principles which have stood the test of experience. These schools, which have to be planned partly according to the exigencies of the site, partly according to the number and character of the rooms required, agree in their main points. Where the same building is to be used both for boys and girls, separate entrances are provided, and the sexes are kept apart, both in school and in the playground. The system of drainage, which must not have any direct attachment to the outside drains, provides for closet accommodation at the rate of three closets per 100 boys, five per 100 girls, and four per 100 
infants. ${ }^{1}$ The upper stories of the school are reached by short flights of stairs, the steps of which are of a height of $5 \frac{1}{2}$ to 6 ins., so as to be climbed without difficulty even by young children; and to prevent accidents, no triangular steps are allowed.

Classrooms.- The most important rooms in a school itself are the classrooms, and every detail of these must be arranged for the benefit and convenience of the children. Quiet is particularly necessary, and the acoustic properties have to be considered from the double point of view of teacher and scholar. It is an unnecessary strain on the former if the voice has to be raised in order to carry to the back rows of the class; while the children follow an oral lesson with great difficulty if speech is resonated and echoed between the walls. The size of the room depends, of course, on the number of children to be accommodated, but the length from the blackboard to the opposite wall should never be so great as to throw any strain on the eyesight of the children in the back row. By the English Day School Code the minimum floor-space per child is fixed at Io sq. $\mathrm{ft}$. (9 sq. $\mathrm{ft}$. in the case of infants), and the minimum area for each room is I $8 \mathrm{ft}$. by $15 \mathrm{ft}$., with a height of $12 \mathrm{ft}$. The cubic space thus allotted to each child in a room I $2 \mathrm{ft}$. high would be only $120 \mathrm{ft}$. -a wholly inadequate arrangement. So small an air-space is soon vitiated, and even with a less restricted allowance, free ventilation is vitally important. This is more efficient if maintained by artificial than by natural methods. In some experiments conducted by $\mathrm{Dr}$ Kerr, the relative merits of these two methods are clearly shown. During the two hours of an afternoon session (2 P.M. to 4 P.M.), the amount of carbon dioxide in a schoolroom was increased from 200 to 700 , taking IOO as the standard of the playground air, while with mechanical ventilation during a corresponding period, the carbon dioxide remained stationary at a level well under 200 .

Associated with the question of ventilation is that of warming the room during the cold months. Open fire-places may answer this purpose in smaller rooms, though their ventilating

1 In boarding-schools a larger number is necessary-I to ro boys, I to 7 girls. 
efficacy is not great, as they withdraw only the lower strata of air. Hot air or steam pipes maintain a more equable temperature throughout the whole room, and can be arranged to assist in ventilation. They possess an enervating effect due to the excessive dryness of atmosphere they produce, but this can be obviated by placing open vessels of water on each radiator. A temperature of between $55^{\circ}$ and $60^{\circ} \mathrm{Fahr}$. is to be aimed at both in winter and in summer; in the latter season protection against the sun may be required.

A cardinal point in a classroom is its illumination. By taking care that no neighbouring house is near enough to intercept daylight, we can ensure plenty of natural light. The main source should be so arranged as to fall on the children from their left-hand side, and thus enable them to do their writing exercises without the annoyance of confusing shadows. Headlights, by producing this bad effect, are undesirable, while any light coming from the front is harmful, owing to the glare in the children's eyes when they look towards the teacher or blackboard. Supplementary windows should be placed either behind or to the right of the class. Artificial illumination has to be so planned as to light the room generally, and also to provide sufficient illumination for each desk. It should fall in such a way as to minimise the difficulty of shadows, and yet to allow a clear view of the blackboard without dazzling the eyes. To avoid this last effect, it is necessary for all sources of artificial illumination to be at such a height above the children as to be outside their field of vision.

The advantages obtained from a well-lighted room can be considerably enhanced by an intelligent scheme of wall-colouring. Light colours are always preferable to dark, and tints which are fatiguing to the eye should be avoided. The most restful and most appropriate are some of the paler shades of green or greenishyellow, and these should be employed for the whole wall, except for a dado of a deeper shade. It is important that the paint or distemper should dry without any shiny surface or gloss, and should be capable of being washed.

The furniture required in a classroom is simple enough, though much has been written as to the best type of desk. 
Single desks, each built on light but strong lines, for one child, have advantages over other varieties arranged to seat two, three, or more children; for, though the former occupy more floor space, they decrease the chances of infection from child to child. In the planning of a desk, two main difficulties have to be met.

The first is the varying heights of children, not only in different classes, but even in the same class. In the next chapter will be described the harmful bodily effects of badly adjusted or unsuitable desks, and here mention need be made only of the fact that the child's comfort requires that careful attention should be paid to the distance between desk and seat, and between seat and platform or ground. Children when writing should not require to stoop to their work, yet, on the other hand, the desks must not be so high that the paper is brought within the proper focal length of vision. For these reasons adjustable desks that can be altered to suit the size of the individual are to be preferred. The seat of the desk, slightly hollowed on its upper surface and tilted at a moderate angle backwards, should be provided with a high, narrow, sloping back to support the body when the child is in a listening attitude. Finally, sufficient space must be allowed for the limbs beneath the desk. A wooden foot-rail adds much to personal comfort.

The second difficulty is to obtain a desk so planned that it is equally suitable for writing, reading, or listening. This triple function is hardly possible, inasmuch as the act of writing requires that the seat should pass beneath the deskan arrangement that makes standing impossible. A choice has therefore to be made on this point from three patterns. Either the seat can pass beneath the desk, or its edge can be vertically beneath the edge of the desk; or there may be a horizontal interval between the two edges. The last arrangement interferes with a correct position for writing; the second is ideal neither for writing nor for standing, but has the advantages and disadvantages of both. The first arrangement is to be preferred, inasmuch as it allows every advantage for both writing and listening, while for reading the child can easily stand at the side of the desk. 


\section{The Hygiene of School Life.}

The standard of school health is to be measured in terms of the physiological and psychological capacities of the average boy or girl; and whenever these powers are either overstrained or under-exercised, we may be sure of some grave defect in the school curriculum. Only too often the simple requirements of healthy childhood are ignored, and a system is followed which results in injury to its subjects. Certain broad conditions of school life can be neglected only at the cost of health, and they are as essential to mental vigour as to bodily fitness. Thus the recuperative hours of rest and sleep must be balanced against the fatiguing periods of work. Recreative intervals, holidays, and vacations must be planned to avoid the danger of mental staleness. The classroom time-table must be arranged to suit the diminishing receptiveness of the brain as the day wears on.

Food.-The condition important above all others is good feeding. It is a platitude that " bricks cannot be made without straw," yet generations of school authorities have tried to produce men from badly fed boys. Only in recent times have they awakened to the fact that their methods are open to criticism. A sufficient supply of good, wholesome, simple food is essential to every child, and without it not only must growth suffer, but the power of mental assimilation cannot fail to diminish. The question of food wears a somewhat different aspect, according to whether the day-school or the boarding-school is considered. Day-scholars include, in addition to the children of the poor, large numbers of those of the middle classes. The latter are probably as well fed as any other school-children, if not better. Their parents are in a position to provide good nourishment, and, as the children take all their chief meals at home, they have the advantage of being directly under their mothers' eyes. With the children in the elementary schools, the position is different. Their feeding is often insufficient, sometimes most insufficient. The effect of this want of nourishment on their learning capacities is obvious, for an ill-fed brain cannot do the work of one that is well fed. These children tire 
quickly when their brains are on short commons, their mental processes work at a low level, memory becomes unreceptive and reasoning is dulled. The folly of forcing instruction on children, who, hungry and ill-nourished, cannot benefit by it, has now been recognised, and by the passage of the Provision of Meals Act, steps have been authorised to remedy the evil. This act, which secures a means of feeding necessitous school-children, establishes in this country a system that has been successfully tried for many years on the Continent-indeed, it is more than a quarter of a century since Paris first began on a large scale to feed elementary school-children at the public cost. The experience in that city has justified the scheme. Of the $£ \mathrm{I}, 250,000$ that the Paris Municipality now spends annually on education, less than one-thirtieth goes to provide meals at the cantines scolaires, but the resulting benefits to the growing intelligence of the children and to the public health are generally recognised as being out of all proportion to the expenditure. We have every reason to suppose that as the English Act is gradually applied throughout this country corresponding advantages will be apparent in our own schoolchildren.

The feeding arrangements at boarding-schools have for long been the subject of adverse criticism. In spite of the increased knowledge that modern physiology has provided of the requirements of the young, the harmful methods of earlier generations have by no means passed away, and schools still exist in which either the food is defective, or the arrangement of the meals is open to objection. Even now, boys, old and young, are allowed to undertake early morning work on empty stomachs. Rising, perhaps, at 6.30 A.M., they may have to attend chapel and first lesson before any opportunity is given them to break their fast. Dr Dukes has laid special stress on the harmfulness of this arrangement, which should be modified even for the senior boys, while the younger ones should have no first lesson at all, but should devote their time, between rising and beginning work at nine o'clock, to breakfast and to half or three-quarters of an hour's play. This change could not fail to be beneficial, and no difficulty would arise in providing 
the full number of hours of class work suitable for boys under thirteen years.

In fixing the times of meals, it is important that practical attention should be paid to the great metabolic activity of children. The wear and tear of their tissues are great, and their cells soon use up available nourishment. Digestion and assimilation are therefore quick. These physiological requirements cannot be met if the meals are served at long intervals. The principal meals of the day-breakfast, dinner, and teamust be supplemented by nourishment in the middle of the morning, and by a further light meal at bed-time. The necessities of the growing body can only thus be satisfied, and further good will result if facilities are given for rest after meals while digestion is actively proceeding.

The principal foodstuff of childhood is carbohydrate, and this should not be stinted at any meal, while the numerous ways in which starchy foods can be presented leave no excuse for a monotonous fare, which is as distasteful to children as to adults. Meat is a necessity, and should be provided at two of the meals. Fat should be eaten more in winter than in summer. Its heat value is great, but children never really care for it.

The place of alcohol in a school diet requires mention. Whatever views may be held as to the value of a stimulant to adults, no one can maintain that alcohol is a necessity for boys, or that it is required as a pick-me-up to restore their flagging energy after exhausting muscular exercise. Throughout the first two decades of life the recuperative powers are extraordinarily buoyant, and, with the help of food and rest, the greatest fatigue soon passes. Boys, unlike men, are not often called on to be at their best at a time when they are physically exhausted by previous work. That the use of a stimulant, even in a comparatively mild beverage such as beer, can result in harm, is undoubted, especially at an age when the animal instincts have first asserted themselves, and are not yet mastered. Therefore alcohol should be rigidly excluded from the school fare. This exclusion would entail no hardship, and, indeed, could be only for the benefit of the boys themselves. 
Work and Rest.- After the question of food, the most important matter is the proper apportionment of work and rest. Work, whether mental or physical, implies fatigue; and, since children are incapable of sustained effort, their weariness must be relieved by frequent periods of rest. The degree of freshness of mind that a child brings into his classroom depends primarily on the division of his hours between sleep and waking; but, as has been conclusively shown by $\mathrm{Dr}$ T. D. Acland, ${ }^{1}$ the majority of the boarding-schools of this country allow their boys, especially the younger ones, too little sleep. The healthy minimum is often curtailed. They rise too early and go to bed too late. The usual practice is to send the younger ones to bed not earlier than ten o'clock; and it is even later before they get to sleep, for they are disturbed by the subsequent arrival of the senior boys of their dormitory. This difficulty would be avoided by separate dormitories for the older boys. The younger should be undisturbed between IO P.M. and 7 or 7.30 the next morning, according to the season of the year. These times allow nine to nine and a half hours of unbroken sleep, which is the amount required by boys under thirteen years. In preparatory and other schools where the younger children are educated, longer hours are necessary, and a boy of eight should spend half his time in sleep (twelve hours), while with each successive year from twenty minutes to half an hour less will suffice. The exact number of hours of rest required by any particular child can be ascertained only by experiment. Allowance must be made for personal idiosyncrasy.

Only the child who obtains sufficient sleep will respond satisfactorily to school instruction; but even with a full allowance, frequent intervals of rest will be required throughout the day, and these must be allotted according to the psychological necessities of alternating effort and fatigue. The most readily wearied mental attribute of children is their power of attention. For a period varying from a few seconds to a few minutes they are able to concentrate their attention on some specific point, but beyond this limit their interest inevitably relaxes, and cannot be again centred on the same subject without an

1 Lancet, vol. ii., 1905, p. 137. 
interval of rest. Each successive effort of attention leaves an increased disinclination to further exertion, until at the end of from half an hour to an hour, according to the age of the child, the mental attitude demanded by the particular subject becomes exhausted. If, after a short rest, a new subject is substituted, a fresh attitude of mind is required, and this will continue without fatigue, but with the same waning attention, almost but not quite as long as before.

Thus each subject wearies the child considerably in respect of that particular subject, and moderately in respect of all other subjects. The store of mental energy is drawn on, until at the end of two or three hours, profitable work is no longer possible, and its attempted accomplishment becomes harmful. A partial but incomplete recovery may be made within a couple of hours or so, and then further productive mental effort may be undertaken, though it will not be on as high a plane as before, and the child will more quickly become tired. Finally, short periods of rest are found to be insufficient to revive the brightness of mind, and the child becomes incapable of further head-work until after a long period of sleep.

These facts have a plain bearing on the arrangement of a school day. The fleeting power of attention is a matter for the consideration of the teacher, who will understand the necessity of claiming the close attention of his class only for short spells throughout a lesson, and will allow it at frequent intervals to fall to a lower level. The duration of individual lessons should be restricted to half or three-quarters of an hour, with an interval of ten to fifteen minutes before the next subject. These breaks should be turned to advantage by allowing the cramped strain of the sitting posture to be relieved by standing, walking, or short physical exercises. It should be remembered that children are naturally restless and little able to endure lengthy periods in one position, and their attention is likely to suffer if they are prevented from occasionally discharging their superfluous muscular energy. Further, it is always difficult to maintain a healthy minimum of carbon dioxide in a classroom, and therefore it is advisable for the children to quit the room, while windows and doors are opened to allow a free flushing 
with fresh air. In a morning session lasting three hours, this ventilation should take place at least once.

Successive lessons in the daily programme must be arranged to employ different mental processes, and no one faculty should be overfatigued while others are left unexercised. Thus, Euclid and geography, or algebra and history, by appealing to reasoning and memory respectively, act as useful foils to each other. A similar choice is necessary in allotting morning and afternoon subjects. The mind is brightest and most retentive in the earlier part of the day, and Sir J. Crichton Brown has emphasised the importance of setting the heaviest mental work before noon. After 5 P.M.-evening preparation or home-work, as the case may be-none but light studies should be demanded. Mathematics and languages should be placed in the morning session, scientific demonstrations, drawing, essay-writing, etc., in the latter part of the day.

The total daily number of working hours which are proper for children of different ages can be stated only approximately, and of course some allowance must be made for the personal factor. The danger, however, of overworking children, or even of children overworking themselves, is very real, and leads more often than is sometimes realised to lamentable results. It is better by far that a child should be taxed below his capacities, until these have been exactly appraised, than that he should incur the risks to mind and health of overwork. The following figures show the total number of hours of school work per week that should be permitted between the ages of five and nineteen :-

Number of Hours of School Work for Children of Different Ages. (Dr Clement Dukes.)

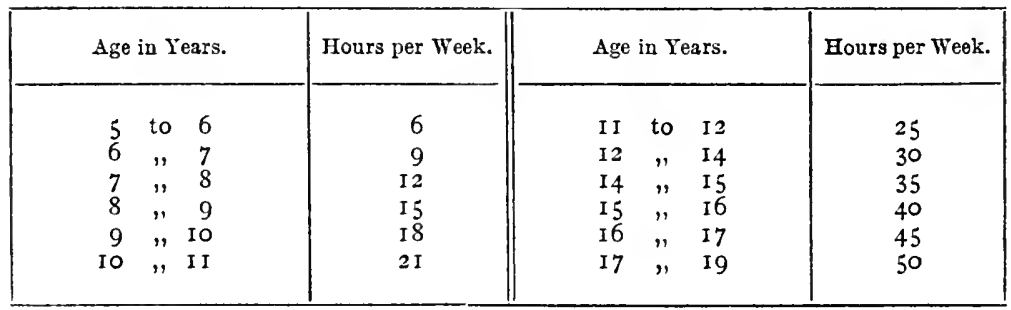


In day-schools the morning session usually lasts three hours, and the afternoon session two hours and a half for each of five days-a total of some twenty-seven working hours in the week. This is a reasonable total, provided there are no homelessons; but, except public elementary schools, few institutions do not require some evening study from their pupils, be it much or little. This is a matter which ought to be regarded from the point of view of the child's health, and not merely from the standpoint of his scholastic progress. Medical opinion is unanimous that a child who has spent the morning and afternoon within the walls of his classroom ought not to be required to carry out additional study in the evening. The harmfulness of the practice is often aggravated by the character of the work set. The progressive fatigue of the mind during the day demands that if evening work is insisted upon, it should be of the lightest quality. In practice these lessons are usually a preparation for the classes of the morrow, and therefore include subjects that require the use of the higher faculties. Only too often the amount of the home-lessons is an index, not of the children's idleness during school hours, but of the teacher's own incapacity; and the more successful the teacher, the less will be the need for out-of-school work.

In addition to the intervals between morning and afternoon school, all children stand in need of regular day or half-day holidays. These breaks are valuable, as they interrupt the otherwise monotonous round of classes, and allow the children to indulge such tastes and hobbies as they may possess. As a rule, two half-days are preferable to one whole day. With regard to the long terminal vacations, little can be said in their defence, either from a physiological or from a psychological point of view. It cannot be maintained that children require some three months every year in which to recover from the strain of the remaining nine; if these were necessary, the methods of the school would be in need of revision. Nor can it be held in these days of competition and hard work, that the schoolmaster, alone of all working mankind, is in need of this prolonged rest. It is, however, a custom, and a custom is never more irradicable than in a conservative profession. One advan- 
tage claimed for the practice is that children are thereby enabled to feel the influence of their homes for long periods ; but apart from the occasionally dubious value of this change, it must be remembered that the more closely schools bring their methods abreast of modern ideas, the less will a purely home environment be required, and the more will the school possess the virtues of home without its almost inevitable educational drawbacks. That the summer vacation is excessively long appears to be tacitly admitted by the practice of imposing holiday tasks, a fashion which robs the interval of its essential value-a complete change of life and surroundings-and allows any good habits of learning imparted at school to be undermined by study attempted without skilled supervision.

Another point that must be considered is the educational relationship between work and recreation or play. Recreation, no less than school-lessons, is educative work : each re-creates the energy for the other. This reciprocity must be remembered in connection with impositions and other punishments. It is a mistake to look on recreation merely as a reward for work well done, and therefore it is wrong to make a practice of punishing a boy by keeping him at work when he should be at play with his companions. The unhappy child, instead of returning to his next lesson fresh and invigorated like his classmates, is more mentally weary than at the beginning of the interval, and more likely than before to repeat his inattentiveness or laziness. It should be considered just as unreasonable to keep a dull boy at work indoors during a recreation interval, as it would be ridiculous to compel an unathletic boy to practice his games while his companions are in school. The dull-witted child needs as much off-time as the bright child-in many cases more.

Apart from these shorter intervals, half-day or whole-day holidays provide opportunities for more systematic exercise, as in football, cricket, paper-chases, etc. The object of these various relaxations should be to cultivate those qualities and powers which, though desirable in adult life, are little likely to be developed by book-work. In addition, those recreations are also of value which not only provide physical exercise in the 
open air, but also directly or indirectly aid the boy's store of information and his methods of observation. Thus the less energetic exercise of walking, if associated with hobbies, such as botanising, geologising, and the many other forms of natural history study, are of the very greatest educational value, and are likely to be of more benefit to certain types of boys than the more violent but less instructive pastimes. For this reason, rules making football and cricket compulsory may perhaps be beneficial to the majority of boys, but should be applied with many exceptions. In this way delicate children will escape undesirable strain, and those whose tastes are a little out of the common will not be compelled to spend their half-days uncongenially. In all school-games that demand vigour, and throw a certain amount of strain on the players, it is very necessary to arrange that boys of approximately equal physical strength are joined in the same and in opposing teams. A child who is put to play with others bigger than himself will soon be exhausted, but rather than give up he is likely to overtax his energy. Serious or even permanent harm may follow.

Dress.-The question of dress will be only very briefly considered, as it has much the same importance in school as at home. The clothing of all children requires to be loose, so that no pressure is exerted on chest or abdomen, and complete freedom of muscular movement is allowed. It is a serious mistake to think that the trunk alone needs protection, and that no harm can result from imperfectly clad limbs. The importance of a warm soft covering for both arms and legs is not sufficiently recognised, and yet one has only to recall the relatively great heatloss suffered by children to appreciate the necessity of conserving this energy. Growth itself implies the consumption of energy, and if a part of the total quantity available is dissipated into the air, less must remain for bodily increase. 


\section{CHAPTER VI \\ MEDICAL ASPECTS OF SCHOOL LIFE}

INTRODUCTION: Hygienic Differences between Home-life and School-life. Infectious and Contagious Diseases-Zymotic Diseases: Spread by Schools ; Infectious Children attending Schools ; Teachers penalised if Infectious Children sent away from School ; Infectious Children and Prize Attendances; Sunday Schools and the Spread of Infectious Fevers; Prevention of Spread of Infectious Fevers in Schools; Health Certificates; Schoolroom Cleanliness ; Exclusion of Scholars; Closure of School; Closure of Infant Department; Closure for different Fevers-Infectious Diseases in Boarding-Schools-Tuberculous Diseases: Statistics of Tuberculous School Children; Infected Schoolrooms; Infectious Scholars; Infectious Teachers-Contagious Skin Diseases. Conditions Dependent on ClassRoom Hygiene: Responsibility for Maintaining Healthy Conditions; Training of Teachers in Elementary Hygiene and Physiology-Bad Ventilation and its Effects-Unsuitable School-desks-Injury to Sight from Unsuitable School Work-Overwork.

\section{INTRODUCTION.}

THE medical problems of school-life are the medical problems of child-life in general, viewed in the particular setting of the school. There is no disease for which schools alone are responsible, and there is no means of prevention or of treatment of ill-health at school except those employed in daily practice. With the exception of the ailments of the first four or five years of life, "diseases of children" and "diseases of school-children" are necessarily synonymous terms in every country where education is compulsory. In the interest of the children themselves, their health should be regarded from the double standpoint of life in school and life out of school, and not from 
the narrow view of school-life alone. It is further desirable that the study of the health of school-children should include a familiar knowledge of the common morbid conditions which arise before the time when educational training begins. Disease weighs most heavily in the first few years after birth, and therefore many children reach the schoolroom already debilitated, if not actually unhealthy. A practical familiarity with such changes is required, in order to safeguard the further development of the child. We cannot doubt that the medical conditions of the youngest school-children - the so-called "infants" of the public elementary schools-are of first importance. By concentrating attention on these, the health of the boys and girls in the more senior classes will cause relatively less anxiety.

Though the complementary relation between home and school must not be overlooked, their essential differences, from the point of view of health, must be recognised. In two particulars especially, school-life stands by itself. In the first place, comparatively large numbers of children are brought into intimate physical contact with each other, both in classroom and playground. Opportunities are therefore numerous for the transmission of infectious and contagious diseases. The second difference is found in the hygienic arrangements of the classroom itself, for if these be imperfect the children will suffer in many ways. Thus the difficulty of providing sufficient fresh air in the confined space of a classroom is great, and bad ventilation will inevitably leave its mark on the general wellbeing of the occupants. The muscular restraints of a schooldesk are unnatural to the restlessness of children, and the resulting fatigue may lead to deformity. Again, the constant appeal that educational methods make to the sense of sight is apt to injure the eyes and to accentuate the ill-effects of deficient vision.

The subject of the present chapter is the medical aspects of school-life in contradistinction to those of home-life, and it will be most conveniently treated under the two heads of (I) Infectious and Contagious Diseases, and (2) Conditions dependent on Classroom Hygiene. 


\section{Infectious and Contagious Diseases.}

The infectious and contagious diseases that are of special importance in school-life fall into three groups-namely, ( 1 ) zymotic diseases (infectious fevers), (2) tubercle, and (3) contagious skin diseases.

(1) Zymotic Diseases. - The fact that schools do materially assist in the spread of zymotic diseases among the child population is beyond question. Sir Shirley Murphy has shown ${ }^{1}$ that more cases of scarlet fever and of diphtheria arise during the periods when schools are open than during the vacations. He states that in the ten years 1895-1904 there were 27.6 per cent. less scarlatina, and 23.3 per cent. less diphtheria in the holidays than in the two months before or after. These figures illustrate the increased risk a child incurs at school, and emphasise the necessity of providing every means to check the spread of infection. In practice, the attainment of this end is beset with great difficulty, partly because of the impossibility of recognising the infectious state of a child sufficiently early to prevent harm, and partly because of the readiness with which infection is spread by books, drinking-cups, and slates, as well as by kissing, coughing, etc. Some circumstances are specially likely to promote the spread of zymotic disease. Thus many cases of slight sore throat are diphtheritic in nature, yet, because of the mildness of the symptoms, parents commonly regard them as not requiring medical treatment, and allow their children to continue their school attendance. Even when the infectiousness of an illness, such as measles, has been recognised, many parents are sufficiently ignorant or careless to permit their children to return to school as soon as the acute symptoms have subsided. These children may be still infectious, and it may well happen that their condition will escape the notice of their teachers. Even should the latter be suspicious on the point, it is not to their advantage to send away a child merely on the chance that he is in an infectious state, because the money-grant to the school is based on the average attendance, and this is not allowed to fall without unavoidable cause. On other occasions,

1 Lancet, vol. ii., p. 406, 1907. 
children qualifying for one of the medals or prizes given for regular attendance may sicken with fever; yet they will continue their attendances at a time when their infectiousness is high, rather than absent themselves and so lose all chance of their reward.

Even should proper preventive measures be adopted to check an epidemic in a day-school, the good results may to some extent be frustrated by Sunday-schools, Band of Hope classes, and school-treats, which may still permit the children to congregate and spread their disease. Sanitary authorities have not the same legal position with regard to these assemblages as in the case of public elementary schools, and cannot compel their closure. Sunday-school authorities may often be relied on to assist in the matter, but even should they decline, the danger is less than in the case of day-schools, because of the smaller attendances and less frequent meetings of the former.

The most careful system of medical inspection or supervision will not ensure a school against these diseases. Their mode of conveyance is so insidious, and their detection in the incubation period so difficult, that sometimes until a zymotic fever has actually broken out in a school, no suspicion of the danger may be roused. This limitation in our powers requires that on the first appearance of an infective disease, prompt and vigorous action should be taken to stop its spread. If its origin can be traced, appropriate measures can be enforced; but until the danger has been localised no means should be neglected to stop probable and possible sources of infection. In the next few pages the precautions that are customarily observed will be considered.

As a preventive measure, health certificates should be required at the beginning of each term, and children who are known to have been brought into possible contact with infection during the holidays should be regarded as probable sources of danger. Sometimes it may be necessary to cut off a street, village, or neighbourhood from all communication with the school. With regard to preventive measures within the school-apart from good sanitary and hygienic arrangementsscrupulous cleanliness of classrooms, cloakrooms, lavatories, and 
corridors is essential. There is no doubt that the condition of the floors is often overlooked, and yet it is in the dust accumulated thereon that pathogenic germs of all kinds are likely to be found. The duty of maintaining cleanliness falls on caretakers and other servants who are not likely to appreciate the importance of this work, which should be carefully supervised. ${ }^{1}$

When one or more cases of infectious disease occur in a day-school, the proper course of action is to send the patients to their homes, and duly to notify the medical officer of health. In accordance with the Public Health (London) Act, I89I, it is the duty of the medical officer, if in London, to inform the head-teacher of the occurrence of an infectious case among the pupils attending his school. The brothers and sisters of such patients should be excluded from further attendance until the expiration of a full period of quarantine, dating from the last day on which they were exposed to possible infection. As regards the remaining members of the class or school, common prudence demands that a sharp watch should be kept for premonitory symptoms, and, in the event of any such suspicious development, the affected child should be temporarily excluded until doubt no longer exists as to the nature of his illness.

Despite every precaution, the first case is only too often the harbinger of many others. When the disease has assumed the proportions of an epidemic, it is very difficult to cope with it successfully. The possible alternative measures are the exclusion of certain scholars, or the closing of the entire school. In the former case, the attempt is made to continue the educational work of the school, while forbidding admission to all those children who are possible carriers of infection. Thus, in addition to excluding patients and individual suspects, an entire class or even a department may be suspended. In outbreaks where the disease is found to be specially prevalent in certain areas, it may be advisable to exclude all children from a certain street or neighbourhood. But even these remedies may fail; and if the disease is found not only to be spreading, but appears

1 A leading authority on public elementary schools has stated that "in one administrative county it is considered quite sufficient to order that the floors of the schoolrooms shall be scrubbed three times a year." 
to depend on the intercourse of the children within the school as opposed to their association outside, the question of closing the school will arise. This step may be strongly urged on a medical officer by the school managers if the daily attendance has become seriously depleted. For the reason already mentioned-that Government grants are dependent on average attendances, and that many absentees will depreciate the value of this assistance-the school authorities may prefer that the whole school should be closed. The remedy, however, is drastic, and should be employed only exceptionally. The fact that the money grant is affected even seriously, is not sufficient justification for the step. In a memorandum of the Local Government Board dealing with this subject, it was pointed out that closure means a serious interference with educational work, and should not be recommended as a routine measure, or until other methods have been tried. The Local Government Board for Scotland has put the matter even more forcibly, and, in a memorandum, has stated that "the fact that many school children are suffering from an infectious disease, and that the attendance is thereby greatly reduced, is not in itself sufficient ground for advising the closure of a school." It should be remembered that cases arising in school may have been infected elsewhere, and that the school may be less responsible than the homes of the children for the spread of the disease. In such instances, the scholars, if dispersed to their homes, may run as great if not a greater risk of acquiring the disease than if they were allowed to continue their attendances, and they will suffer the additional disadvantage of losing some weeks of instruction. The experiment has been tried of closing some of the schools in an infected district, and merely excluding suspected scholars from others in the same locality, but the comparative results go to show that the more drastic remedy is no more efficacious than the other. Drs Newsholme and Pakes sum up the whole matter in the following words:-

"School closure is indicated: (I) if measures of exclusion of individual children have failed to prevent the continued and increasing spread of an infectious disease among school children, the number of cases of this disease being in excess 
of the incidence of the same disease in the rest of the district, and of the number of cases among the children attending other schools; (2) if, by reason of the absence and exclusion of a large number of children the attendance at school is greatly reduced."

The second contingency, though not in accord with the statement quoted earlier from the memorandum of the Local Government Board for Scotland, is probably one commonly accepted, though the interpretation of "greatly reduced" must be left to the individual medical officer.

On all occasions of infectious outbreaks, the youngest children suffer the greatest mortality, and therefore, of all the school departments, the infants' should be closed with the least hesitation. It has been customary to shut it for a period of three weeks during an epidemic of measles, if the proportion of children affected exceeds 10 per cent. of the whole number. For diphtheria a slightly longer interval is required (four weeks); while for scarlet fever, exclusion of suspects is generally sufficient.

During the period when the school is closed, a thorough cleaning will be required, to rid the rooms and their furniture of infection. The floor and desks should be well scrubbed with soap and water, and the walls cleaned and whitewashed. All books that have been in recent use will need to be disinfected by heat; though, by preference, they should be destroyed.

With regard to the re-admission of cases, proper care must be taken to prevent the attendance of any child until the expiration of a full period of quarantine, calculated from the last day of possible infection. Further, it is desirable that the clothing of the child should be free from any infectious taint.

Infectious Diseases in Boarding-Schools. - In boarding-schools an outbreak of infectious disease is an even more serious matter than in day-schools. The school drainage, water-supply, milksupply and laundry are occasional sources of infection, but often the disease is brought from outside, either from houses in the neighbourhood-perhaps by day-scholars-or from a distance, as, for example, from the boys' homes. The question of closure requires careful consideration, for, by despatching the pupils to 
their homes, infection may possibly be carried to many parts of the country, as well as to railway carriages and other conveyances. In these schools it is often possible to decide whether the centre of infection is within or without the school, and in the latter event closure is not likely to be required, and the infected area can be easily placed out of bounds. In the former case closure should be resorted to only exceptionally and as a last measure. By prompt isolation of every case and of each suspected case, the outbreak should be kept within limits. This isolation must be complete, and must be extended to all the sick-room attendants as well as to the patients. Parents, however, do not all regard a school epidemic in the same light, and therefore it is wiser to give them the option of withdrawing their children should they be over-apprehensive for their safety.

2. Tuberculous Diseases.-Apart from the zymotic fevers, there is another disease, the infectivity of which requires that healthy children should be protected from intercourse with infected subjects. Tuberculous disease occurs at all ages of school life, and most commonly manifests itself in the lungs, bones, or glands. Recently much attention has been given to statistical estimates of its prevalence in school children. Thus Dr Newsholme has estimated that in school children not more than $I$ in 300 is tuberculous. As a result of an examination of I670 children at two large elementary schools in London, only eight cases of definite pulmonary phthisis were discovered, though fourteen others presented suspicious signs-a total percentage of only $\mathrm{I} \cdot 3$. These figures suggest that tuberculosis is not nearly as common in school communities as was at one time feared; yet even so low a percentage as $\mathrm{I} \cdot 3$ means, in the London schools alone, a total number of tuberculous children amounting to about 7000 . On the other hand, it is necessary to remember that most of these and allied investigations have been made on school children well enough to attend school, while there has been no allowance made for those who are invalided either temporarily or permanently. It is among these that tubercle will be most commonly found. Tuberculous children very quickly ail, and are usually kept at home by their 
parents, or are sent into convalescent establishments or homes for cripples. An exact estimation of the importance of the disease in school health must obviously take into account not only those children who are still healthy enough to continue their attendance, but also those whom the disease has either incapacitated or killed after they have attended school. If this were carried out, it is possible that the prevalence of tubercle among children of school age would be found to be materially higher, and the percentage thus obtained would be more in harmony with the experience of those who see large numbers of school children in children's hospitals. Further, allowance would have to be made for the difficulty encountered even by the most experienced in detecting the earliest stages of pulmonary phthisis before sufficient signs are present to render it, in the current term, "revealed." Pending the collection of more inclusive statistics, we should be imprudent to underestimate the incidence of a disease which is known to be one of the greatest scourges in the years before and after schooling. Children should receive every possible protection from the disease, and we should omit no precaution for their safety.

The principal dangers, so far as schools are concerned, come from three directions-(a) from dirty schoolrooms; (b) from other children already infected; $(c)$ from tuberculous teachers or other officials.

(a) The remarks on school cleaning that have already been made in connection with the zymotic fevers apply equally to tubercle, for abundant proof exists that dust and dirt may be frequently contaminated by tubercle bacilli. Special attention is required to the floor, which is peculiarly liable to harbour these germs wherever expectoration occurs or saliva is showered in coughing or sneezing. Fresh air is very necessary in the prevention of phthisis, and though open windows cannot always be arranged during the day, this ventilation is possible out of school-hours. The average caretaker, however, having swept a room at the end of an afternoon session, is careful to seal all windows till the morning, lest any fresh dust should be blown in from the street and appear to undo his labour.

(b) The grave danger that arises when tuberculous children 
are allowed to associate in class with healthy schoolmates can be avoided only by the separate education of those capable of transmitting the disease. In some cases it is necessary to isolate a child from his fellows, even though the diagnosis is as yet uncertain, and a salutary regulation has long existed in the London elementary schools, by which a child who is subject to coughing or spitting is excluded from attendance. A larger problem is raised by the suggestion that children from tuberculous homes should not be allowed in ordinary schools. Though their presence brings an added risk to their fellows, the difficulties in the way of excluding them make the practical observance of this precaution hardly possible at present. Nevertheless it behoves both school medical officer and teacher to keep a vigilant eye on such children. They should receive special attention as to their personal cleanliness, with a view to ensuring so far as possible their freedom from infectiousness. They should also be kept under constant observation for the earliest signs of tuberculous disease. If any ground for suspicion arises, the child must not be allowed to continue at an ordinary school, but should be transferred, if the arrangement is possible, to an invalid or an open-air school.

A tuberculous teacher is no less a danger than a tuberculous schoolfellow. The most careful and elaborate efforts to secure health at school may be stultified if the children are allowed to come into daily contact with this peril. A teacher necessarily handles the slates or papers of each member of the class, and these articles form a ready channel for the conveyance of germs. The importance of disease among teachers as a factor in the spread of tubercle depends on the frequency with which they themselves are affected by this disease. Unfortunately, evidence is not wanting that the teaching profession is one peculiarly subject to tubercle. In the United States the death-rate for female teachers was, in 1900, nearly twenty per cent. higher (256 to 2 I 5 per 1000 deaths) than for all females; while in Denmark the mortality from tuberculosis among those teachers who were insured was twice as high as among the insured of the general population. Though this lamentable disproportion has not been proved to exist in this country, Dr Tatham has drawn 
attention to the fact that the mortality from consumption among teachers is high in England and Wales, and therefore the risk in our own schools is a real one. In Paris, on the other hand, tuberculosis is stated to be relatively rare both among male and female teachers. Whatever may be the exact position in this country, it is clear that the health of the school teacher is of importance to the health of the children. It is therefore only right that steps should be taken for the medical examination of teachers, in order to detect those who are tuberculous; and, if positive evidence is obtained, the sufferer should be removed from duty, and not allowed to resume work until there is reasonable ground for supposing that all risk has passed. In London it is customary to take these teachers away from their work for at least twelve months, and this period is not too long. By a logical extension of this practice as regards the teaching staff, medical examination should be undertaken in the case of all minor officials, such as caretakers, whose duties provide them with opportunities for spreading disease.

(3) Contagious Skin-diseases. - These diseases differ in importance from the zymotic fevers and tubercle, in that they can hardly be termed inimical to life, and indeed are often associated with good health. Their strictly medical importance is therefore not great; yet from the scholastic point of view they include some of the most vexatious maladies. Ring-worm, impetigo, scabies, and head-lice (pediculosis), are all very common indeed among the poorer children, while less common are favus and molluscum contagiosum. Certain chronic inflammations of the eye are also contagious. All these affections are "dirt conditions," that is, they are dependent on personal and domestic uncleanliness; and, owing to the fact that they are transmitted by personal contact, children suffering from them must be excluded from school. The mothers of these children, however, are often, as might be expected, careless and indifferent. They will make little or no effort to carry out the proper cure, so that an unnecessarily serious loss of school attendance is the consequence. Impetigo, scabies, and pediculosis should require only a short absence from school, but the more intractable ring-worm and favus may 
well mean many months of enforced absence, especially if treatment by X-rays cannot be obtained-indeed, about one-tenth of all school absences in London are due to ring-worm alone. In spite of these difficulties, there is no alternative but to exclude all cases rigidly, and to refuse re-admission until a complete cure has been effected. Though special ring-worm schools were established under the Poor-Law Authorities, a satisfactory arrangement has yet to be devised for public elementary schools. A small step in this direction was taken by the London County Council, which in I 906 opened a school for children with favus.

\section{Conditions Dependent on Classroom Hygiene.}

We have next to consider the ill-effects that may result from deficient hygienic arrangements in the schoolroom itself. The principal factors responsible for these common evils are four in number: (I) Bad ventilation; (2) Desks unsuited to their occupants; (3) Injury to sight from improper use of the eyes; and (4) Overwork. Each of these conditions means bad hygiene, and therefore implies deficient supervision on the part of some responsible official. The question is: Who is the individual responsible for maintaining healthy conditions in a classroom ? Only comparatively recently has it been recognised that the health of school children is as much the concern of the teacher as is the state of their minds, but in many schools it still remains nobody's business to assure the proper surroundings. The head-teacher is not often in the classroom, and his time is filled with matters of administration-curriculum, discipline, parents, and the like; while the assistant teacher, whose time is spent entirely in the classroom, is fully occupied with teaching, and further is brought into too close a relationship with the scholars to be able to detect in them any gradual loss of health and energy. It is not difficult to understand how a teacher may, for example, fail to recognise the increasing vitiation of the air in his classroom as the half-hours pass, and may tolerate an atmosphere so impure as to be offensive to a newcomer. With an increased knowledge of hygiene and physiology among teachers, a more intelligent regard for the condition of the 
schoolroom will result, and the bodies of children will no longer be made to suffer in order that their minds may be crammed. The training of teachers in the elements of these subjects has already been enforced in certain foreign States, and has recently been made obligatory in Scotland. When a similar regulation is framed for England and Wales, an important step will have been taken in school hygiene.

(I) Bad Ventilation.-Ventilation has already been considered, and we have seen how very rapidly the quantity of carbon dioxide increases in the air of a classroom when only natural ventilation is provided. When children are compelled to breathe a vitiated atmosphere for a number of hours daily, injurious results must follow, and the fact that this harm is insidious in action and not easy to recognise, increases the gravity of the danger. Children who have been exposed on a single occasion to impure air may strikingly show the effects in their flushed cheeks and bright eyes, and in their complaints of frontal headache. But when this poisoning is repeated day after day, little is to be noted beyond a general ailing. Such children lack energy and appetite ; they are always tired, and complain much of headache. These symptoms are not characteristic, for they also occur under other circumstances; but in any particular case where an originally healthy school child begins to ail, it is advisable to make enquiry as to the usual conditions of the classroom atmosphere. As a general rule, it may be stated that in those school children whose sight is not defective and who are without organic disease such as enlarged tonsils, recurrent headaches usually result from the bad ventilation of their classrooms.

(2) Desks unsuited to their occupants.-The importance of a well-planned desk has already been insisted on. When the requirements in this direction are neglected, and children are placed at desks too low or too high for them, or are seated three or four of unequal size at a single long desk, harm is likely to result. The most serious effect is lateral curvature of the spine. This, though less likely to develop in strong children, is not uncommon in the less vigorous, who acquire this deformity 
as a result of frequently assuming an asymmetrical position. Such an attitude is often adopted when a child begins to write; but it is also made use of as a resting posture, especially if the desk is unprovided with a suitable back, or when the child, standing to read, transmits the weight of the body through one leg only. This asymmetrical position of rest, though normal at first, tends with frequent repetition to become exaggerated, and the result may be a serious and permanent deformity. Another effect of using an unsuitable desk is round-shoulders. This is apt to appear in those who require to stoop to bring their eyes within focal length of the desk. Both these deformities are preventable, and they could in many cases be averted by judicious supervision on the part of the teacher. Whenever a child is seen to assume a bad posture, the fault should be at once corrected. If there is frequent need of interference, it may be taken for granted that there is some definite defect either in the child or in the child's immediate surroundings, and if this cannot be discovered and remedied by the teacher, the assistance of a doctor should be obtained on the child's behalf.

(3) Injury to Sight.-Evidence of another series of effects of bad school hygiene is found in the frequency with which the eyesight of children suffers as the result of school work. The constant use of the eyes in educational work necessarily taxes the sight, and unless precautions to limit this strain are taken, defective vision is likely to result. In a child's eye the optical axis is relatively shorter than it is in later years, and accommodation has therefore to be freely used in near work. This action, if employed beyond normal limits, is liable to throw a strain on the ciliary muscles of the eyes. Young children who are set to do fine work, whether in reading small print, writing, or sewing, are being taxed beyond their powers, and they usually acquire the harmful habit of bringing their work well within the proper focal length of ten inches, so that not only will their accommodation be in over-action, but the object will be held so close to the eyes as to be seen only by squinting. Severe strain on the ocular muscles results; headache and neuralgia are the common consequences, and the sight may be permanently affected. To prevent these troubles, fine eye-work 
should be removed from the curriculum of younger children. Older children should be prevented from acquiring the habit of stooping at their work. Hypermetropia and astigmatism are both common defects in school years, and do much harm if allowed to remain uncorrected by suitable glasses. Myopia is least common in the youngest classes, but gradually increases in frequency as the school training is continued. Thus, observations on a series of children of all school ages in Brussels showed that only 5.5 per cent. of the youngest pupils were myopic, but this figure increased until in the oldest classes no fewer than I 8 per cent. were affected. The frequency with which school life produces myopics is a serious matter. Their number can be reduced by careful attention to conditions of illumination, shape of desks, size of print, etc., and by consideration of the child individually, so as to prevent the habit of under-focussing the eyes when at work.

(4) Overwork.-Finally, we come to the question of overwork. Though the pathological condition of brain-fever (meningitis) cannot be produced, as is the popular belief, by overwork alone, nevertheless the functions of the brain can be, and frequently are, disturbed and injured by too long or too close an application to school studies. As would be expected, the greatest harm is produced most readily in young children. "To spend too much time in studies, is sloth," said Bacon. We can interpret this as meaning that the capacity for productive work is restricted, and that all efforts to stretch this limit are, to a greater or less extent, wasted. This capacity varies with the child and with the nature of the work; and as was shown in the last chapter, class-work very quickly induces mental fatigue. If the wearied activities are still compelled to attempt the assimilation of more learning, the attention can be roused only with great difficulty, mistakes (as in spelling or arithmetic) multiply, and simple relations of fact cease to be appreciated. Information forced into the overburdened head under these circumstances is lost within a few hours or days, and the time and labour of the effort are wasted, though they might have been employed to some useful purpose. Children may be the victims of unintelligent teachers, or of rigid curricula, or of the 
examination-fetich, each of which ignores the physiological and psychological powers of childhood, and attempts to make school instruction bear premature fruit instead of first allowing it to put forth leaf and flower in due course.

Since I 895 , when a Joint-Committee of the British Medical Association, the Sanitary Institute, and other bodies, sitting under the chairmanship of Mr Douglas Galton, reported on the mental and physical conditions of 100,000 children, attention has been prominently drawn to the evils of overwork and to the changes produced thereby. It is well to distinguish between the effects of overwork on a single occasion, and of overwork persisted in for weeks or months.

In the former case there may be some headache, but children, as a rule, are merely tired-both in body and in mind. They are without energy for vigorous play after their release from school, and after perhaps a restful interval devoted to some quiet relaxation, are soon prepared for their beds, and, by morning, have probably slept off their fatigue.

In chronic overwork the changes are more profound, and are recovered from only slowly. Such children show the exhaustion of their brains by many signs which require to be distinguished from those indicating natural stupidity. They lose that restlessness of motion which characterises the healthy child, and the brightness and gaiety of their spirits disappear. They are disinclined to join in vigorous games, and become listless and dull. When spoken to, they reply in monosyllables, and they are unnaturally obedient. At the same time the colour goes from their cheeks, their eyes grow dull and heavy, a constant frown suggests worry, their muscles feel flabby and their gait is without elasticity. A noticeable undermining of their general health becomes apparent, and with it their intelligence loses its alertness.

Teachers who are unable to interpret these changes notice only the increasing hebetude of their pupils, and, misjudging its cause, may try to spur them abreast of their schoolfellows. They bring them to the front row of the class to keep them under closer control ; or, perhaps, impose additional work, and restrict their recreation-intervals-intended remedies, which 
can lead only to further disaster. At home these children will make frequent complaint of headache, especially on their return from afternoon school. Very commonly, they begin to talk in their sleep, repeating fragments of their lessons; and not infrequently the habit of somnambulism is acquired. If at this stage they are medically examined, they will be found to present clear indications of nervous and muscular exhaustion. They do not willingly assume bodily attitudes requiring an expenditure of much energy. Their heads, instead of being held erect, decline forward or lean to one side, and if their arms are extended in front of them, the fingers and hands droop at the wrist.

The only remedy for this condition, which is seen in girls as well as in boys, is release from some or all school work, according to the severity of the symptoms. With ordinary care, and especially with careful parents and observant teachers, no child should present more than the earliest indications of chronic overwork before he has the conditions of his school-life revised. Children who have suffered recent illness are more prone than others to develop signs of overwork, and they therefore require to be specially closely watched during the few weeks after their return to school. 


\section{CHAPTER VII}

\section{MEDICAL INSPECTION AND SUPERVISION OF SCHOOL CHILDREN}

DUties of SchoOl MEdical OfFICERS: The Education (Administrative Provisions) Act, 1907; The Board of Education and the Medical Inspection of Schools; Outline of the Scheme ; Duties of the School Medical Officer; School Hygiene; Inspection of Children; Routine Inspection and Detailed Examination; Prevention rather than Cure; Frequency of Medical Attendance ; Trivial Febrile Attacks ; Underfed Children; Half-Timers. Methods of Inspection: Medical Requirements; Preliminary Assistance by School Nurse; Routine Inspection-General and Anthropometric-Eyes and Ears-Mental Capacity and Speech-Mouth and Throat; Special Examination; Schedule of Medical Inspection; Annual Reports. Common Pathological Conditions of School. Children-Dental Caries -Enlarged Tonsils and Adenoids-Swollen Glands in the Neck -Lateral Curvature of the Spine-Stammering and its Treatment. INVALID SCHOOLS: Their Object; Children suitable for Admission; Open-air Schools. MEDICAL SUPERVISION OF BOARDING-SCHOOLS : Physique of Children of the Upper Classes ; Unsatisfactory Hygienic Conditions of Boarding-Schools ; School Sanatoria ; Required Amount of Invalid Accommodation; Sickrooms; Attendance and Duties of Medical Officers ; Preliminary Examination of Boys on first entering School.

\section{The Duties of School Medical Officers.}

THOUGH attempts to deal with the conditions of health in schools were made in France some seventy years ago, the problem of medical inspection of children was not recognised as of real importance until the concluding years of last century. In England public interest has been aroused even more recently, and in this respect we have lagged behind most civilised countries. Medical inspection has been in practice for some years both in leading countries such as Germany and Austria, and in minor states such as Portugal, Servia, and Argentina, 
but in England we are only now embarking on the undertaking. The report in I 895 of a Joint-Committee of the British Medical Association, the Sanitary Institute, and other bodies, showed that the medical inspection of school children was feasible, and, though no concerted action followed, various isolated authorities, with the help of specially appointed officers, undertook the duty of a partial inspection of their school children.

The observations that were thus made by the medical officers-prominent among whom has been Dr James Kerr in Bradford and subsequently in London-disclosed a lamentable amount of preventable disease, and the demand for a systematic and permanent medical inspection of all school children found expression in 1907, when the second International Congress on School Hygiene met in London. In the latter part of the year an Act of Parliament-the Education (Administrative Provisions) Act, I907-was passed, whereby the medical inspection of children in public elementary schools was included among the duties of local education authorities. Section 13 ( 1 ) $b$ of this Act deals with the matter in the following terms:-

Section I3 (I) The powers and duties of a local education authority under Part III. of the Education Act, I902, shall include:

(a) Power to provide for children attending public elementary schools, vacation schools, etc.

(b) The duty to provide for the medical inspection of children immediately before or at the time of or as soon as possible after their admission to a public elementary school, and on such other occasions as the Board of Education direct, and the power to make such arrangements as may be sanctioned by the Board of Education for attending to the health and physical condition of the children educated in public elementary schools :

Provided that in any exercise of powers under this section the local education authority may encourage and assist the establishment or continuance of voluntary agencies and associate with itself representatives of voluntary associations for the purpose. 
This section was ordered to come into operation on the first day of January 1908.

The duty of carrying out the inspection has been laid on the local education authorities, whom the Board of Education advises and supervises as to the manner in which this work should be performed. In a memorandum of 22nd November $1907,{ }^{1}$ the Board gives general guidance as to the minimum amount of inspection required, and outlines the scheme that is to be adopted by local authorities throughout the country. School medical officers will be required to work in harmony with this scheme, the details of which may be summarised as follows :

The whole subject of school hygiene is regarded as resting on the broad basis of public health; therefore the work of medical inspection is carried out under the direct supervision of the medical officer of health. In county areas the county medical officer deputes the actual execution of this work to medical colleagues or assistants (men or women), who are either appointed specially for the purpose, or are local medical officers of health. In county boroughs the town council instructs its medical officer of health to advise the education committee, and makes him responsible for the work or for its supervision; while in non-county boroughs and urban districts which are local authorities for elementary education similar arrangements are recommended. In each case the functions of the school medical officer may be exercised by a medical officer of health, a poor-law medical officer, a private practitioner, or, as occasion requires, a specialist. Preference should be given to those who are adequately trained in State Medicine, have some definite experience of school hygiene, and have enjoyed special opportunities for the study of diseases in children.

The success of medical inspection must depend on the sympathetic assistance of the school teachers, for they are in a position to furnish data respecting each child, to carry out some portion of the inspection, and to apply the principles of hygiene to school life. Other subsidiary but important agents

1 "Medical Inspection of Children in Public Elementary Schools" (Circular 576). 
are the school nurse and the health visitor, who by acting as links between the school and the home are able to visit and give counsel in the home, and to enlist the sympathy and educate the sense of responsibility of the parents.

As to the medical inspection itself, not fewer than three inspections are necessary during the school life of a child. The first should take place at or as soon as possible after admission to school; the second at or about the third year (say the seventh year of age); and the third at or about the sixth year (say the tenth year of age). A further inspection is desirable immediately before the child finally leaves, though in some areas it may be best for the third inspection to be made at this time. At the first inspection it is desirable that one or both parents should be present, for not only can they facilitate the examination and provide information, but the medical opinion of the inspection can be given directly to the persons most nearly concerned.

The above is a general outline of the scheme put forward by the Board of Education. The plan is intended for adoption in all parts of the country, so that a uniformity of results may be available for comparative purposes; but the working details will, of course, vary according to the particular needs and circumstances of each area or group of schools.

That the medical inspection of school children will be successful, and will repay its cost many times over, will not be doubted by anyone acquainted with the medical conditions of public elementary school children; but the system, being an innovation, can hardly fail to raise objections in many quarters, and the school medical officer will require to proceed with tact until his beneficent duties become better understood. A certain amount of difficulty may be anticipated from some school teachers, who will resent the introduction into their classes of a new force which can hardly fail to influence the old routine methods of education; but this opposition must inevitably succumb as the teachers themselves grow better acquainted with the conditions of health and ill-health. A less reasoned and therefore more obstinate prejudice is likely to come from the parents themselves, some of whom will be averse from permitting any medical examination of their children. These difficulties, 
whether made within the school or outside, might jeopardise the success of the inspection by withdrawing the co-operation of the two classes of observers-teachers and parents-whose assistance is so important; and the prejudice, on which all such difficulties are founded, will require tactful handling. The practical good effected by inspection will be measured by the frequency with which physically defective children are submitted by their parents to medical treatment, and here again the apathy of so many of the lower classes on such occasions will require vigorous rousing by the medical officer, and in this task the help of the school nurse or health visitor will be invaluable.

With regard to the actual work of a school medical officer, his duties fall under two heads: (I) the sanitary or hygienic arrangements of the school; and (2) the inspection of the children. The former includes not only the condition of the school buildings, but the internal hygiene of the classrooms, and the prevention of the spread of infectious diseases. These subjects have already been dealt with in the two previous chapters. In the next pages special attention will be given to the second duty.

Medical inspection is not intended to be a detailed medical examination, but merely an expeditious investigation to ascertain the presence or absence of those defects and diseases which commonly interfere with schooling. It is intended as a means of "determining the fitness of the individual child for school life." In the majority of cases this point can be settled very speedily, but, in a few, a more elaborate investigation than that in routine use will be required. Thus, to ascertain the fitness of a child for school life, it will only exceptionally be necessary to palpate the abdomen, or to make a special examination of the lungs. The medical officer's task will consist, therefore, of: (a) routine inspection; and (b) detailed examination. The former must not be curtailed on any occasion; the latter will depend on the requirements of the particular case, and on the discretion of the medical officer. Anyone experienced in sick children should be able to detect abnormal individuals almost at a glance, and by a rapid inspection to ascertain which, if any, 
of the commoner diseases he has before him. The statement by the Board of Education, that the inspection of each child should not occupy on the average more than a few minutes, has been much criticised as suggesting a perfunctory inspection; but it can safely be said that, with practice, and with the assistance of a nurse, all the requisite information can in most cases be obtained within the space of a few minutes-probably not more than ten.

Medical inspection must eventually be concerned rather with the detection of the earliest signs of ill health before any serious harm or gross change has been produced, than with the discovery of children already too far diseased to be restored to normal conditions. Prevention should become the essence of the system. To obtain the maximum benefit the medical officer should be available at other times besides those when one of the set inspections is in progress. Daily medical attendance is the rule in many countries, and another desirable arrangement is the one in force in Sweden, where the school doctor pays a weekly visit to watch the children at their classes, interviewing them and being interviewed by any who wish for advice. A daily morning visit, at the hour when the school is assembling, is desirable, in order that every child whose condition has aroused the suspicion of a teacher or nurse, can have its illness at once provisionally diagnosed before possible infection has spread to others. At these morning visits, any children who have been absent for the few preceding days owing to sickness, would be submitted to the doctor before resuming their places in class. As a matter of practice, it is desirable that all convalescents should be seen in this way; for, apart from the ignorance of parents as to the real nature of a supposed trivial febrile attack, there are reasons, as we saw in the last chapter, which may induce the continued attendance of a sick child, or his premature return to school. As it is not to the interest of school teachers to allow an apparently unimportant illness to interfere with regular attendance, mild examples of specific fevers, especially diphtheria and scarlatina, might be allowed to attend, while cases of measles, whoopingcough, or mumps, might be excluded, not at the first suspicious 
indications, but only later, when the diagnosis was beyond doubt. The proper remedy for this evil, and for that of prize attendances by sick children, is, of course, the institution of epidemic grants such as were formerly made. Absence from school on account of ill health should still count as attendance for the purposes of the grant or prize; and thus neither the teachers nor the children would be penalised for illness, and the risks of infection to the school as a whole would be diminished.

The more intimately the doctor is associated with his schools, the better qualified will he be to understand the influence of the curriculum-work, recreation, exercise-on the children as a body, and to detect the causes of ailing health in individuals. Two kinds of children will require his special attention-the underfed and the half-timers. Under-feeding has been discussed in an earlier chapter, and the harmfulness of mental effort on a hungry child emphasised. Half-timers are children above the age of twelve (or eleven in agricultural districts), who, under certain conditions, are partially exempted by law ${ }^{1}$ from school attendance, and divide their time between factory or other work and education. This exemption is largely claimed in areas devoted to the textile industry, but in many leading cities, including London, Liverpool, and Glasgow, half-time has been abolished by by-law. ${ }^{2}$ This course has been adopted owing to the frequent complaints that children who have been at work from early morning to mid-day are too exhausted to profit by instruction in the afternoon, and that, as a further result of their factory-work, there is a moral deterioration and a weakened sense of discipline, which can only tend to infect the whole-timers. The health and physical development of these juvenile workers is likely to be adversely affected, and in districts where half-timers are allowed, the school medical officer will require to pay special attention to them, and to be on the alert for indications of overstrain. It should, however,

' Education (School Attendance) Amendment Act, I 899.

2 Since 1903, County and Borough Councils have had powers to pass by-laws regulating the employment of children for wages or profit, especially as regards the minimum age, maximum number of working hours, and exclusion from occupations harmful to health or dangerous to morals. 
be remembered that the mere fact that a child's day is divided between school and work is not necessarily injurious, provided the conditions of each are satisfactory and the total demand on the child's energy is not excessive. The well-known experiments of Mr Charles Paget, M.P., who divided the boys in his village school into half-timers put to garden-work, and whole-timers attending school only, resulted in the former excelling the latter in every way. Such an outcome, however, is hardly possible where the half-timers spend their working hours, not in the open air, but in a factory or mill, where high pressure of work and large expenditure of energy are demanded. The conditions of this child-labour are at present under the consideration of a Departmental Committee of the Home Office, and it is probable that the legislation on the subject will undergo revision before long.

\section{The Methods of Inspection.}

Medical inspection should be carried out on the school premises during school hours. For convenience and expedition, it is desirable that a special consulting-room should be provided for the school medical officer. This room must be well lighted, artificially warmed, and provided with a table, chairs, a small couch of simple fashion, weighing-machine, height-register, and Snellen's test types. Facilities for washing are essential, and among other requirements are: lotion-bowls, metal or wooden tongue-depressors, thermometer, measure-tape, magnifyingglass, depilation forceps, etc. A dental mirror may be useful, and a small steriliser may be needed for the tongue-depressor. Apparatus for bacteriological examination of the throat and other parts should be at hand.

To avoid waste of time, each child should be made ready for the doctor by the school nurse or other assistant, who should obtain and record such preliminary details as name, age, address, previous illnesses, and condition of clothing. An experienced nurse may also be relied on to register the height and weight, and to ascertain the child's temperature, if this point is likely to be required. When many children attend at 
the same time, it is desirable that they should be prepared in a waiting-room, and admitted to the doctor, one by one, accompanied by their parents-by preference the mothers, as fathers are singularly useless, both in facilitating the examination, and in providing details in the previous medical history.

A routine method of examination should be adopted. The schedule, issued by the Board of Education (see p. 138) admitcedly represents the minimum required, and for completeness and greater accuracy a more detailed investigation is desirable. The suggestion that "the child need only, as a rule, have its clothes loosened," is likely to interfere with the detection of many conditions. Sometimes not even the presence of so grave a condition as tuberculous spinal disease can be ascertained by this superficial inspection, and often a mere glance over the whole body and limbs will afford information which, in the interests of the child, must not be overlooked. For a satisfactory inspection, the child must be stripped well below the waist, back and front, and it is highly desirable that the feet and legs should also be uncovered. To obviate any risk of chill, a wrap of some washable material may be thrown over the shoulders. With the more senior girls of the sixth and seventh standards, it may be preferable to uncover only a limited part of the body at a time; but a complete inspection is nevertheless required, and is not often objected to.

The routine examination may be conveniently made in four stages :-

I. General and Anthropometric.-If the child has already been undressed, the weight and height should be taken in this condition; but if the clothes are worn, the height should be measured without boots, and the weight taken in ordinary indoor garments without boots. If any child is found below the average in either of these records, the cause of this deficiency must be carefully sought. The chest measurement at the level of the nipples-except in older girls-should be recorded in each of the extreme positions of inspiration and expiration; the difference between these represents the expansion, and is a more valuable indication than either of these measurements alone. The general physical condition must be noticed, especi- 
ally as regards nutrition and muscular development. Anæmia and any peculiarity in the type of face suggestive of disease should be looked for, while paralysis and involuntary muscular twitches will be readily detected. The shape of the chest is next observed, and pigeon-breast and rickety deformity noted. The limbs are examined for any wasting or undue shortening, and the lower extremities for the presence of knock-knee, bandy-leg, or flat-foot. Any swelling suggestive of chronic bone disease is looked for, and the spine very carefully inspected for lateral or kyphotic curvature. The degree of bodily cleanliness must be ascertained, special attention being given to the hands, feet, and scalp. Skin troubles, including verminous heads, may be sought at this stage. Ringworm requires very minute search for its detection; and, as an examination of the whole scalp is needed, a preliminary search may be made by the nurse, by whom every suspicious scurfy area should be brought to the doctor's notice. If any doubt exists as to the condition of a head, a few hairs should be removed by a depilation forceps and examined microscopically.

2. Eyes and Ears.-Examination should next be made for external diseases of the eyes and ears. In the eyes note is taken of any marginal blepharitis, conjunctivitis, ptosis, corneal opacities and nebulæ, squint, nystagmus or cataract; while in the ears the presence or absence of a discharge is of chief importance. At this point it will probably be convenient to test the acuteness of sight and hearing. The former may be approximately estimated by test-type; the latter can, in young children, as a rule be tested only by simple methods, and the doctor's voice is likely to give more satisfactory results than the ticking of a watch. In regard to hearing, and to a less extent to seeing, the teacher or mother may be able to provide important evidence from their own observations.

3. Mental Capacity, Speech.-Mental capacity can hardly be estimated within the brief period of a medical inspection, unless there is some marked deficiency, and therefore the brightness or dulness of a child must be judged with the help of the teacher's experience in the schoolroom. Two points in the history that must be enquired into are epileptic fits and chorea. The 
mental condition is to be tested by the power of attention and the quickness of response; while the various signs of overstrain should be looked for.

Should some mental dulness be discovered, the important point is to decide whether the deficiency arises from natural stupidity or from disease of the special senses. Large numbers of children are stupid because they cannot hear their lessons, or cannot see clearly enough to read their books or the blackboard. The causes of artificial stupidity are to be sought in the eyes, ears, or throat.

In investigating capacity of speech, it must be remembered that a child often requires much coaxing before his tongue can be loosened, and that the mother or nurse can be of material assistance in this connection. The special points to be noticed are stammering, lisping, and mispronunciation of consonantsthe last-named being ascertained when the child tries to repeat test-words spoken to it.

4. Mouth and Throat.-Lastly, attention should be given to the mouth and throat. The condition of the teeth is of great importance-their cleanliness, the presence of caries, irregularities, or malformations-and it is desirable to distinguish between the state of the temporary and of the permanent dentitions. Secondary inflammations in the gums and jaw may be found. The tonsils must be inspected, and, if thought necessary, a digital examination of the naso-pharynx made. The habit of mouth-breathing should be enquired for, as well as snoring at night, headache on waking, and the other indications of obstructive breathing. Finally, the submaxillary and cervical lymphatic glands should be palpated, and note made of the region in which any are felt enlarged.

The above inspection will elicit practically all the facts that are likely to bear directly on school life, and therefore the medical investigation need not go beyond these limits, unless there are special reasons to the contrary. Among such reasons would be symptoms suggesting disease in some organ (e.g., cough, palpitation, abdominal pain), while the presence in the medical history of rheumatism or recurrent sore-throats should direct the attention to the heart. Occasionally it may be 
Schedule of Medical Inspection.

I.-Name

Address
Date of Birth, ${ }^{1}$

School

II.-Personal History :-

(a) Previous Illnesses of Child (before admission).

\begin{tabular}{|c|c|c|c|c|c|}
\hline Messles. & $\begin{array}{l}\text { Whooping } \\
\text { Cough. }\end{array}$ & Chickenpox. & $\begin{array}{c}\text { Scarlet } \\
\text { Fever. }\end{array}$ & Diphtheria. & $\begin{array}{c}\text { Other } \\
\text { Illnesses. }\end{array}$ \\
\hline
\end{tabular}

(b) Family Medical History (if exceptional). ${ }^{3}$

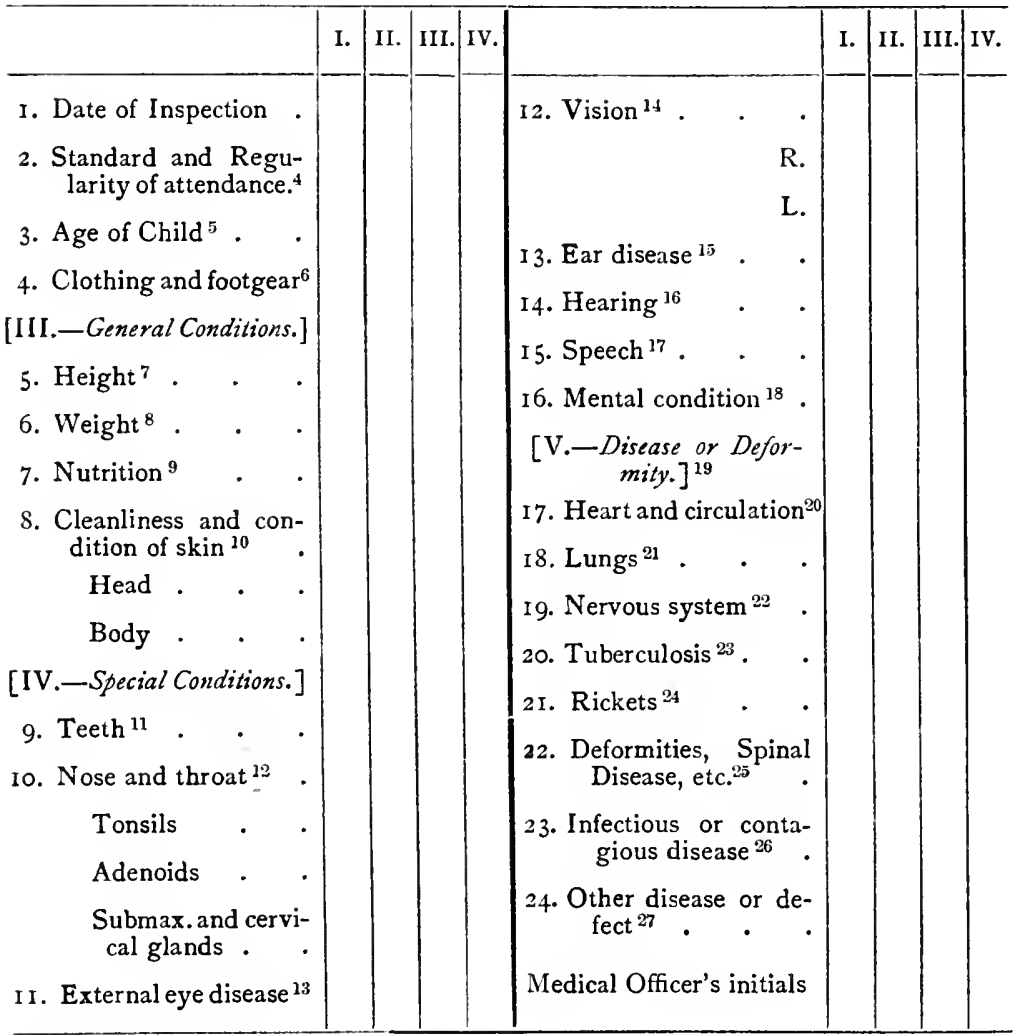

General observations.

Directions to Parent or Teacher. 


\section{Notes for Inspecting Officer.}

Referenec

Number

of Note.

1. Date of birth to be stated exactly, date of month and year.

2. "Other illnesses" should include any other serious disorder which must be taken into account as affecting, directly or indirectly, the health of the child in after-life, e.g., rheumatism, tuberculosis, congenital syphilis, small-pox, enteric fever, meningitis, fits, mumps, etc. The effects of these, if still traceable, shoulù be recorded.

3. State if any cases of, or deaths from, plithisis, etc., in family.

4. Note backwardness.

5. Age to be stated in years and months, thus, $5 i^{4} \frac{1}{2}$.

6. Insufficiency, need of repair, and uncleanliness, should be recorded (good, average, bad).

7. Without boots, standing erect with feet together, and the weight thrown on heels, and not on toes or outside of feet.

S. Without boots, otherwise ordinary indoor clothes.

Height and weight may be recorded in Euglish measures, if preferred. In annual report, however, the final averages shonld be recorded in both English and metric measures.

9. General nutrition as distinct from muscular development or physique as such. State whether good, normal, below normal, or bad. Under-nourishment is the point to determine. Appearance of skin and hair, expression, and redness or pallor of mucous membrane, are among the indications.

10. Cleanliness may he stated generally as clean, somewhat dirty, dirty. It must be judged for head and body separately. The skin of the body should be examined for cleanliness, vermin, etc.; and the hair for scurf, nits, vermin, or sores. At the same time, ringworm and other skin diseases should be looked for.

11. General condition and cleanliness of temporary and permanent teeth, and amount of decay. Exceptional features, such as Hutchinsonian teeth, should be noted. Oral sepsis.

12. The presence or absence of obstruction in the naso-pharynx is the chief point to note. Observation should include mouth-breathing; inflammation, enlargement, or suppuration of tonsils; probable or obvious presence of adenoids, polypi; specific or other nasal discharge, catarrh, malformation (palate), etc.

13. Including blepharitis, conjunctivitis, diseases of cornea and lens, muscular defects (squints, nystagmus, twitchings), etc.
Reference

Number

of Note.

14. To be tested by Snellen's Test Types at 20 feet distance ( $=6$ metres). Result to be recorded in the usual way, e.g., normal $\mathrm{V}_{\mathrm{f}}=6$. Examination of each eye (R. and L.) should, as a rule, be undertaken separately. If the $\mathrm{V}$. be worse than $\frac{6}{4}$, or if there be signs of eye strain or headache, fuller examination should be made subsequently. Omit vision testing of children under 6 years of age.

15. lncluding suppuration, obstruction, etc.

16. If hearing be abnormal, or such as interferes with class work, subsequent examination of each earshould be undertaken separately. Apply tests only in gencral way in case of ehildren under 6 years of age.

17. Including defects of articulation, lisping, stammering, etc.

18. Including attention, response, signs of overstrain, etc.

The general intelligence may be recorded under the following heads:- (a) Bright, fair, dull, backward; (b) mentally defective; (c) imbecile. Omit testing mental capacity of children under 6 years of age.

19. Under the following headings should be inserted particulars of diseased conditions actually present or signs of incipient disease. The extent of this part of the inspection will largely depend upon the findings under previous headings.

20. Include heart sounds, position of apex beat, anæmia, etc., in case of anything abnormal or requiring modification of school conditions or exercises.

21. Including physical and clinical signs and symptoms.

22. Including chorea, epilepsy, paralysis, and nervous strains and disorders.

23. Glandular, osseous, pulmonary, or other forms.

24. State particular form, especially in younger children.

25. Including defects and deformities of head, trunk, limbs. Spinal curvature, bone disease, deformed chcst, shortened limbs, etc.

26. Including any present infectious, parasitical, or contagious disease or any sequela ex. isting. At each inspection the occurrence of any such diseases since last inspection should be noted.

27. Any weakness, defect, or disease not included above (e.g., ruptures) specially unfitting child for ordinary school life or physical drill, or requiring either exemption from special branches of instruction, or par. ticular supervision. 
advisable to examine for conditions such as rupture, undescended testicle, varicocele, or phimosis. When the inspection requires to be amplified to an examination, the procedure will, of course, be that of an ordinary clinical examination-respiratory system, circulatory system, digestive system (with abdominal examination), nervous system, genito-urinary system, and lymphatic system.

For convenience of reference, the complete schedule of medical inspection (with notes for the inspecting officer) issued by the Board of Education is appended (see pp. 138, I39). The four columns are intended for the four inspections during school life. It is suggested that this schedule should be printed on cards, to facilitate reference. All entries should be regarded as confidential, and when a child moves from one school to another, a copy of the schedule should be transmitted to the new inspecting officer.

Every school medical officer is required to make an annual report at the close of each calendar year to the local education authority on the schools and children under his superintendence, and this should be printed for distribution, and two copies sent to the Board of Education. The report should deal chiefly with the conditions and circumstances affecting the health of the children in the elementary schools of the district, and should contain, inter alia, statistical records of the number of children examined, re-examined, and under medical supervision; the nature and result of the examination; the number of visits paid to classes; the number and character of the diseased conditions found at certain age periods; particulars as to blind, deaf, defective, and epileptic children ; the medical advice given as to prevention of conditions inimical to health, and the remedy of diseased conditions; action taken, etc. Of secondary importance would be systematic comparisons with standard and local records of individual and collective measurements, and characteristics of the children in each school.

Common Pathological Conditions of School Children.

Perhaps the half-dozen commonest pathological conditions which are met with in school children are: (1) Dental Caries; 
(2) Enlarged Tonsils and Adenoids; (3) Swollen Glands in the Neck; (4) Lateral Curvature of the Spine; (5) Chronic Heart Disease ; and (6) Tubercle. The last named has already been dealt with in the previous chapter. Heart-disease has no special features that need detain us here ; but the four remaining conditions are of special importance to the school medical officer, and deserve separate consideration. Yet another defect-though, strictly speaking, not a pathological one-that is very common among school children is some imperfection of speech, such as stammering, stuttering, or lalling. These form serious obstacles to a child's progress, and as the means for their relief are not widely known, a short account of the defects and their treatment may appropriately conclude this section.

(I) Dental Caries. - It is the exception to find a set of healthy teeth among the children of the poor. Some fifteen years ago $\mathrm{Mr}$ Denison Pedley examined the teeth of more than 300 school children who passed through the wards of the Evelina Hospital for Sick Children. Of these, only fifty-five had healthy sets of teeth. The remaining 250 possessed, on an average, six decayed teeth each. The lamentable condition disclosed by these observations has since been found to be general among school children.

In the report for 1907 of the Medical Officer (Education) to the London County Council, mention is made of an examination of 700 children at the time of their admission to school; 680 of these showed obvious dental caries, and of these over 300 had more than five decayed teeth each. In the out-patient department of a children's hospital there are probably more medical conditions arising in school children from this single cause than from any other. Its effects are widespread and insidious. Apart from its interference with the proper mastication of food, dental caries produces a long chain of secondary results, the symptoms of which and their connection with the teeth cannot be too clearly recognised by the school medical officer. These effects may be divided into the two groups of : $(a)$ local conditions in and around the mouth ; and $(b)$ general or constitutional conditions.

(a) Among the former the commonest are toothache, 
neuralgia, foul breath, inflammation of the gums, and alveolar abscess. Further, the septic state of the mouth, together with the constant absorption of septic material from the stumps, may lead to an inflammatory enlargement of the tonsils, and a similar change is very common in the lymphatic glands beneath the jaw. (b) The pus that is being continually discharged from the suppurating stumps is mixed with each mouthful of food, and swallowed to the stomach. Dyspeptic symptoms-pain after food, "stomach-ache"-are therefore very frequent, and are intensified by the habit that develops of bolting meals imperfectly chewed. There is consequent loss of appetite, the child is disinclined for his food, and his growth may be hindered. Simultaneously, the chronic absorption of toxic matter from the mouth and stomach induces a general debility, and the patient becomes anæmic and listless, complaining often of headaches, and perhaps developing some twitchings of the face or other habit-spasm. In this state he can profit but little by the school instruction which he is compelled to undergo. Among the most serious complications that have been attributed to dental caries are epilepsy and tubercle. In some cases where the former has been observed in children with caries, the attacks have undoubtedly been seen to abate and even to cease after removal of all decayed teeth. The connection with tubercle, though difficult of proof, is probable, seeing that tubercle bacilli have been found in carious stumps, and would probably be capable of ready absorption, especially if the pulp-chamber were exposed.

As to the cause of this decay, dental surgeons are not unanimous. Rickets and improper feeding in infancy are usually regarded as two important predisposing factors, while acid fermentation of food in the mouth is widely accepted as the direct cause; the acids eroding the enamel and exposing the dentine beneath. From a preventive point of view, it is safe to assume that dental caries is least likely to appear where the teeth are kept clean; and therefore there is urgent need to spread a knowledge of the virtues of the tooth-brush among the classes where this utensil is a curiosity possessing, perhaps, some reputation, but no real existence. Dental hygiene is a subject that can be appropriately taught in school, and by the 
regular practice of the so-called "tooth-brush drill" cleanly habits may be inculcated.

The treatment of dental caries is a question rather for the dental surgeon than for the school medical officer, but mention may be made here of the excuse so often offered by negligent mothers when their attention has been drawn to the condition of their children's mouths-that decay among the milk-teeth does not matter. This extenuation is wholly indefensible, for caries in the temporary dentition often spreads to the first permanent molar (appearing about the sixth year), and may also seriously affect the germ of an underlying permanent tooth.

The dental conditions of the poor are so bad, and are responsible for so much ill health, as to deserve special arrangements for their amelioration. It is to be hoped that before long the medical officers of schools will be given the assistance of dental surgeons, who by periodic examination and treatment of children's mouths will be able to effect an improvement in school health of great benefit.

2. Enlarged Tonsils and Adenoids.-This condition, which is itself the result of some cause or causes at present imperfectly recognised, though probably dependent on bacterial infection of the nose, mouth, or throat, produces a series of secondary changes which are the outcome of obstructed breathing. The airway through the nose being diminished or completely blocked, mouth-breathing is resorted to, and the child gradually acquires the type of face that is characteristic of this habit. The mouth hangs open and the lower lip becomes full and coarse; the nose is poorly developed and the nostrils small. The expression of the face is dull and unintelligent, the palate is narrow and highly arched, the eyes are often puffy and the voice thick with a nasal twang. As a later result deformity of the chest may be produced, and the child will present a greater or less degree of pigeon-breast.

The obstruction to breathing is most pronounced during sleep. Snoring and heavy breathing are usually observed, and sleep is fitful and disturbed. When the partial suffocation is most acute, the child will often be seized with night-terrors, 
or may pass his water into his bed. The effect on his general health is usually marked. He grows listless and mentally heavy, and is always tired and subject to headaches, especially on waking in the morning. At school he is dull and inattentive, and these faults are accentuated, if, as is often the case, inflammation spreads along the Eustachian tubes and sets up deafness. Many children are thus rendered mentally defective.

The treatment of the condition is simple. When there is evidence of naso-pharyngeal obstruction, the surgical removal of the tonsils or adenoids or both is required. Not every case, however, of enlarged tonsils needs operation, and if there is no obstruction it may be sufficient to attend to the general health and the cleanliness of the mouth; but it should be remembered that the unhygienic conditions of life of many public elementary school children are calculated rather to accentuate than to improve the condition.

3. Swollen Glands in the Neck.-This is a particularly common condition, and was found by Dr Kerr in no less than 75 per cent. of a series of 1670 school children. This frequency is an expression of the fact that cervical glandular swelling results from many conditions, among which are some of the commonest diseases of children. Excluding the acute specific fevers (scarlatina and diphtheria), the common causes which are to be sought in any school child are carious teeth, enlarged tonsils, impetigo of the face, skin diseases of the scalp (including pediculosis), and tuberculous infection. By bearing in mind the fact that these enlarged glands most usually mean septic absorption either from the interior of the mouth or from the skin of the head or face, we can usually trace the source of the trouble without difficulty. Pediculosis is often distinctive, in that the enlarged glands lie at the back of the neck, and are smaller and harder than those produced by most other conditions. Finally, it is worthy of note that for some time after measles, the cervical glands may be enlarged and readily palpable.

4. Lateral Curvature of the Spine.-This, the commonest deformity of school children, has already been mentioned (p. I22), and need be referred to again only to emphasise the importance 
of its early detection, and to summarise the school conditions which mostly contribute to its production. These are: (I) desks of unsuitable size; (2) bad illumination of the classroom, and (3) short-sight, both necessitating improper positions for writing; (4) lop-sided attitudes in sitting ; (5) standing in the "at-ease" position; (6) suspending sachels always from the same shoulder. In its prevention and early cure, the medical officer requires the intelligent co-operation of the school teacher and the gymnastic instructor.

5. Stammering.-Among the most important of the common nervous defects of childhood are stammering, stuttering, and lalling. Stammering is due to inco-ordination of the muscles involved in articulation. Stuttering results from repeated contractions of the muscles concerned in forming some initial syllable. Lalling is baby-speech, in which wrong consonants or vowels are employed and the pronunciation of the words is slurred. Each of these defects is curable, and we must therefore ascertain the principles underlying their treatment.

The mechanism of speech is twofold-vocalisation and articulation. The former, which is produced by the vibrations set up in a column of air passing over the vocal cords, depends on the movements of breathing, on the vibrations of the vocal cords, and on the resonating cavities in the head and in the neck. By its help the five "physiological" vowel sounds are produced, namely, ee, a, ah, o, and oo. Articulation is produced by totally different means. The current of air from the lungs is interrupted or released by the muscular action of the lips or of the tongue at one of three situations-the so-called "stoppositions." In the first stop-position the lips are suddenly parted or brought together so that one of the following consonants is uttered-P, B, W, M. The second stop-position is formed by pressing the tongue against the hard palate; the consonants that now result are $\mathrm{T}, \mathrm{D}, \mathrm{L}, \mathrm{N}, \mathrm{R}$ (and $\mathrm{Sh}$ ). The third stop-position is nearer the back of the mouth, and is used to form the consonants $\mathrm{K}, \mathrm{G}$ ( $g$ irl), and Y.

In the majority of cases the nature of the defect is essentially a want of harmony between the mechanisms of vocalisation 
and of articulation - the child is unable to pass either smoothly or quickly from the one to the other. In almost all cases the difficulty lies not in articulating, but in vocalising, and therefore our first step in treatment must be to teach the child how vocalisation differs from articulation. We must then make him give special attention to his vowel sounds, and, in order to increase his command over them, we should begin by teaching him to take steady breaths and to control his expirations. When he has acquired this power, he must be exercised in the different vowel-sounds.

The consonants are to be learnt in three groups, namely, the "voiceless" consonants $\mathrm{P}, \mathrm{F}, \mathrm{Th}$ (thin), S, Sh, T, and K, which are without any vocal element; the "voiced" consonants $B, W$, $\mathrm{V}$, Th (thou), Z, Zh, D, L, R, G, and $\mathrm{Y}$; and the voiced nasal consonants $\mathrm{M}, \mathrm{N}$, and $\mathrm{Ng}$. The important point in learning this classification is that the vocal element in the last two groups should be given their full value. Thus, in pronouncing the three words, brown, prim, and mind, the child must give the full vocal character to the initial consonants of the first and last words-bur-own and um-ind; but the second word, beginning with a voiceless consonant, can be pronounced only as pr-im.

When these consonants have been learnt by the aid of single words, the child should begin to practise sentences. In doing this, his attention must be specially directed to vocalisation. There is no better way of enforcing this precaution than by making him intone in clerical fashion. He will then be compelled to attend rather to his voice than to his articulation. His earliest sentences should be made up of words whose initial consonants are all either voiceless or voiced. In order to acquire facility in the use of the various stop-positions, he should be given sentences to practise which contain the consonants produced at the several positions-thus, for the second position, a sentence such as "Not till late did she return."

These are the simple lines on which stammering is to be treated. Frequent practice is essential, and the exercises are to be selected according to the particular difficulties of speech in each case. By testing the child's vocalisation and articulation 
as soon as he comes under treatment, we shall have no trouble in ascertaining wherein lies his defective power. In cases of lalling, the letters that cannot be pronounced must be found out by making the child repeat the vowels and the consonants. Each of the latter must be tested as an initial letter, as a terminal letter, and as a middle letter.

\section{INVALID SCHOOLS.}

Among children of school age there will be a variable number suffering from some physical defect which incapacitates them from ordinary school life, or makes it desirable either in their own interests or in those of their healthy comrades that they should be trained apart in special schools. Thus blind or deaf children obviously require very special methods of education; paralysed or crippled children cannot fairly be exposed to the rough-and-tumble ways of ordinary public elementary schools; and tuberculous children must be prevented from conveying their infirmity to others. Special schools have therefore been established for the physically defective, and in London there are at present over twenty such centres, containing some I 800 children. Admission is granted only after medical examination, and the training is modified to suit the feebler powers of the invalid scholars. The children are conveyed to and from school in special ambulances, which have become familiar sights in the poorer parts of London.

These invalids include both curables and incurables. The majority of them suffer from one of three diseases-tuberculous bone or joint disease; paralysis ; or heart disease (congenital or acquired). Among other conditions represented are deformities and pulmonary tuberculosis; while the attendance is sanctioned of a few children without definite disease, yet too delicate for ordinary school life.

The want that such schools are intended to fill in large cities, and the benefit conferred by them, are undoubted, but they are still too restricted in number to be used by more than a small percentage of the invalids. A strict selection of cases for admission has therefore to be made, and it is obvious that those cases should receive pre- 
ference which seem best qualified to derive good from the schools. The greatest value possible is to be obtained by choosing those physically defective children who are likeliest to survive to adult years, and to be capable of earning their own living. Less benefit will come from selecting children who, though likely to attain adult years, will be partly or wholly dependent on others; while the least value will be obtained by educating children who are obviously or probably doomed to early death. A child with infantile paralysis should, for example, be preferred to one whose heart is hypertrophied and dilated; or a boy with an amputated leg, rather than one with tuberculous spinal disease.

Tuberculous children are less likely to repay the cost of special training than many other invalids, if their daily home surroundings continue unaltered and the school itself is not specially arranged with a view to the modern treatment of tuberculosis. For this reason, the adoption in this country of the system of Wridschuler, which combines education with openair treatment, promises to confer much good on this group of invalid children. The original open-air school was founded in 1904, at Charlottenburg, near Berlin, with the object of adding to school-training the benefits of open-air and good food. The children, who come out from the town at an early hour and remain throughout the day, receive two to three hours instruction daily, and spend the remainder of their time either in open-air shelters, or among the surrounding pine trees. Their meals, which are frequent and nutritious, are prepared on the premises, and a careful medical supervision of their progress is maintained. This experiment proved so successful, that in 1907 the London County Council established the first open-air school in this country-at Bostall Wood, near Plumstead. In the following year three schools were opened -at Dulwich, Kentish Town, and Shooter's Hill. Now that this system of combined medical and educational treatment has been started in London, other towns are following suit, with benefit not only to the sickly children themselves, but to those in health who are thus freed from a possible source of infection. 


\section{Medical Supervision of Boarding-Schools.}

Though the Education (Administrative Provisions) Act does not apply to the schools of the upper and middle classes, the feeling is growing that an inspection of the hygienic conditions prevailing at many boarding-schools would reveal a condition of affairs which can be remedied only by regular medical supervision. Apart from questions of unhygienic school buildings and dormitories, a surprising amount of physical deformity is known to exist among the children, even of the wealthiest. In a statistical record of the physical conditions of 1000 boys at their entrance to one of the great public schools, Dr Clement Dukes has published ${ }^{1}$ figures which disclose a remarkable condition, both as regards the general growth of the boys and acquired deformities. No fewer than 47 I out of 1000 were below average weight, 365 below average height, and 423 below average chest measurement, the comparison being to the average figures obtained by $\mathrm{Mr}$ Roberts for over 7700 public school boys and others of the most favoured classes. The numbers of their acquired deformities, excluding those that were only slight, were as follow :-

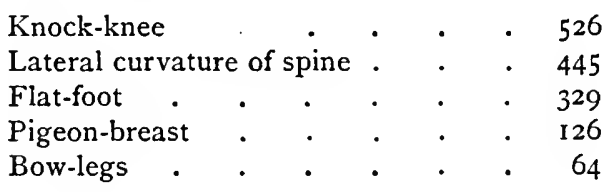

Further, 208 had some form of defective sight, and II 2 were mouth-breathers.

If this deplorable condition is as general among the boys of other boarding-schools-and there is no reason to suppose that the children admitted to this particular school are below the average of other public school boys-the necessity for close medical supervision throughout the whole of school life cannot be denied; and it is equally clear that with so many delicate inmates, no school should be allowed to neglect a proper observation of the principles of school hygiene. This desirable alteration can be attained only when public opinion insists on

1 Lancet, 24th August 1907, p. 512. 
a medical supervision of every boarding-school, both as to the general hygienic arrangements of the buildings and to the individual health of each scholar. Only the largest schools could afford the exclusive services of a medical officer, and therefore, in the majority of cases, the duties must fall on a general practitioner in the neighbourhood. The former arrangement has perhaps the disadvantage that the clinical practice of even a large school is not great, and there is a tendency for the medical officer to fall into a groove. The practitioner, on the other hand, has his knowledge furbished in his daily rounds, and further, he is more likely to become acquainted with outbreaks of illness in the neighbourhood, and so will be in a position to take the earliest precautionary measures to protect the school.

Whichever plan is adopted, the duties to be performed are the same: and these we shall now consider. Some of the medical problems of boarding-schools have been dealt with in the two previous chapters, and need not again be referred to; but there are some additional points that require consideration. The medical supervision of boarding-schools has been specially studied by Dr Dukes, ${ }^{1}$ whose long experience has placed him foremost among the authorities on the subject, and his views will be embodied in the following section.

The ideal arrangement for the treatment of illness is a school sanatorium, though this may not be practicable in small establishments. The sanatorium should be exclusively reserved for sick boys and their attendants, and it must be isolated from the other school buildings. It should consist of two portions-one for infectious and suspected infectious cases, the other for noninfectious cases; and the separation between the two should be sufficiently complete to prevent the possibility of boys passing from one to the other. The rules for isolation must be made to apply not only to the patients, but to all servants, etc., brought into contact with infection, and therefore the sanatorium must be provided with sleeping and living accommodation for the attendants, who should not be allowed to mix with any other members of the school. The block must be under the

${ }^{1}$ Health at School, C. Dukes, M.D., 4th ed., 1905. 
immediate authority of the medical officer, who alone should have the right to grant admission to visitors.

As regards the amount of invalid accommodation required, provision has to be made for the average number of cases of ordinary illness, and also for the possible emergency of an epidemic, when, in a few days, a large number of boys may require accommodation either as patients or suspects. In a memorandum of the Medical Officers of Schools Association, the requisite accommodation has been calculated at 20 per cent. of the total beds in the school. This provision would give the medical officer reasonable means of coping with all but the most extensive epidemics. On any occasion when more than 20 per cent. of the boys were invalided, it is probable that so many of the inmates of one at least of the school-houses would be infected, that their isolation could be satisfactorily maintained in their own house. The older the boys the smaller is their susceptibility, and therefore the accommodation for them can be planned on a smaller scale. A sufficient provision will usually have been made by allowing beds for 20 per cent. of the boys under thirteen years, and only i 5 per cent. for those over thirteen years.

When a sanatorium is beyond the resources of a school, reliance must be placed on sick-room accommodation. This room should be chosen with an eye to its special use, and not merely because it happens to be one not needed for other purposes. It must be suitable in size, light, and ventilation; and must be so situated in the house as to reduce to a minimum the chance of infection spreading beyond its door, as well as to prevent the inmates from establishing personal communication with their comrades. As in the case of a sanatorium, the attendants must not be allowed to mix with others in the school, if any infectious case is under their care. Wherever possible two sick-rooms should be provided, as the patients are likely to continue in isolation for some considerable time after the acuteness of their illness has passed, and during this period it is desirable for them to have a special room for night use and another as a day-room.

Daily morning attendance is incumbent on the medical 
officer, so that all cases of indisposition can be submitted to him, and appropriate measures adopted at the outset - by admission to the sick-room, exemption from class-work, etc. In this way minor ailments will not be neglected, and possible serious complications will be avoided. In the event of serious illness, the medical officer is probably the most suitable official to communicate with parents, who are apt to be over-anxious about the condition of a child away from home, and will be most readily assured by a note in the doctor's hand.

In these morning visits the medical officer is able to keep himself acquainted with the general sanitary conditions of the school, and to improve his personal relations with the boys. Schoolboys when ill are by no means too ready to present themselves for medical treatment, and a successful medical officer is one who succeeds in winning their confidence so that they feel they have in him a friend and kindly adviser. Specially should he keep a sympathetic eye on delicate boys or those with some physical defect ; while he can often do much to smooth the path of the backward or mentally dull. The latter are in some cases held in little favour by their class-masters, who, perhaps not altogether unnaturally, may be inclined to judge boys too exclusively by their classroom reputation. The doctor is more likely to make allowance for a deficiency which is the fault of no one, and by putting himself to the trouble of gaining the child's goodwill, he may be able to lighten the unhappiness which is only too often the undeserved meed of the boy who cannot shine at his work.

In every boarding-school a complete medical examination should be made of each boy on his entrance. This examination is not different from that described above for children in elementary schools, but may with profit be made more searching. Thus early information will be obtained of any defects so that remedies can be applied without delay. The physical powers of the boy can be roughly estimated, and any necessary restrictions in the matter of games decided on, subject of course to amendment as further experience may suggest. The results of this initial examination should be placed on record-a separate card for each boy is the most convenient for future 
use-together with the family history and the previous medical history (including the date of last vaccination). These family details, especially if they are open to suspicion, are not always provided by the parents; for example, the fact that a boy comes from a consumptive family, may be intentionally withheld from the doctor by the relatives. Such culpable reticence may easily result in harm to the child, and no wise parents should object to take the medical officer into their confidence, especially as he, no less than a private medical adviser, would regard such communications as confidential.

At the beginning of every new term, each boy should be reexamined and the results again recorded. His freedom from infectious disease, and from exposure to infection during the recent holidays, must be attested by a certificate brought on his return. 


\section{CHAPTER VIII}

\section{THE TRAINING OF CHILDREN}

Introduction. Educational Methods of the Present Day. The Material : Animal Instincts; Habit-formation; Attention; Enthusiasm ; Sensitiveness to Blame; Desire for Sympathy. THE METHODS: Methods must conform to the Process of Evolution; Stages in Training ; (I) Spontaneous Training; Infant Education; Sensorial Training: Motor Training; Infant "Play"; Habits of Life; Speech ; (2) Disciplinary Training; Obedience; Prizes and Punishments; Religious and Esthetic Influences; Schools; Use of Leisure; Relation between Home and School; Minimum Age for School Attendance; Scholastic Instruction; (3) Adolescence; Relaxation of Authority; Ideas of Altruism and of Community of Action; Intellectual Training ; Inductive and Deductive Reasoning; Puberty. ThE SPEcial Training of GIRLS : Comparison between Girls and Boys from the point of view of their Training; Co-education of the Sexes; Rules of Health; Phenomena of Sex; School Training; Domestic Economy; Childrearing; Influence of Teachers on Girls. ABNORMAL Types OF Children: The Selfish; The Passionate; The Cruel; The Lying; The Precocious; The Neurotic.

IN the training of children we must keep before us two specific aims of outstanding importance. The first of these is to assure their bodily health. This implies, not only that our educational methods must themselves be harmless, but that we must actively instruct children in the ways of healthy living. Without health a man is a burden to himself if not to the community; he falls short in the performance of his duties, and he is, perhaps, a danger to his own or to coming generations. Our second object must be the moral training of children, by which their untamed animal instincts are to be shaped and their characters formed. Our purpose must be the cultivation of self-respect and a proper sense of responsibility towards 
others. Though these qualities do not mature until after adolescence, they need careful tending from the earliest years. The child, therefore, must be regarded from two points of view. From the one aspect we see him as an individual whose ideas of self require our special attention. From the other aspect we must look on him as a unit in the school community who must conform to the good of the many. Though the social obligations involved in the corporate life of the school have in late years come to be regarded by many educationalists as of chief importance, we must always remember that a child who does not understand his duty to himself is not likely to appreciate his duties to others.

Young children, with their experiences limited almost exclusively to self, have no real conception of common interests, and are necessarily non-moral and non-ethical. They are not capable of understanding the necessity of denying their own desires and impulses. The first steps in their moral training can therefore be taken only by exacting implicit obedience. This control should not be relaxed until repeated practice has ingrained the primitive canons of conduct as habits of thought and of action. Later, when a sense of right and wrong has been instilled and conscientiousness has begun to develop, dogmatic authority can be slackened, and appeal made to the child's own sense of honour. The final stage should witness a still further remission of authority, inasmuch as the end in view must always include the formation of independence and of self-reliance.

Not until health and character exist as sufficiently sure foundations on which to build up intellectual training, should we give prominent attention to those subjects a knowledge of which will serve to fit the child for a working place in the world. The trained mind is not the one that has absorbed the greatest amount of information, but the one that has found the exact use of its own contributory senses, and is able, with their help, to form independent and sound judgments. To attain this end, a long pupilage is necessary, and it must be planned on natural lines that will call into play all the channels of sensory information and the responsive muscular actions. As an important item in 
this training, we must supplement the experiences of the young mind with observations made by others-that is to say, by the study of books. The environment of the child will thereby be widened to an extent which is possible by no other means. At first the mental training should not concern itself with the probable adult occupation of the child, but should be devoted only to imparting that knowledge which is of general applicability and is necessary to everyone. Later, a somewhat more special bias may be given to the instruction; but not until the general training has been completed, should the future occupation of the child be allowed to cast its shadow on his educational course.

Before we fill in the system of training above outlined, we must briefly glance at the methods in vogue at the present day in the majority of our schools.

Education, as Herbert Spencer has said, is the subject which involves all other subjects. Therefore we may conclude that it must always take cognisance of changes and advances in every branch of knowledge. In other words, education must never be stereotyped in its methods, but, always keeping itself abreast of modern thought, must be constantly assimilating new ideas, and accommodating itself to modern progress. As a matter of fact, we find that the system of to-day is dominated by the still powerful influence of Herbart. His teachings not only succeeded in excluding from attention many earlier systems, such as those of the Realists headed by Comenius-whose admirable views, according well with twentieth century ideas, have remained unapplied for nearly three hundred years-but they have monopolised the field against all later comers. Herbart's methods were founded on a strictly psychological basis, but, having been completed before the scientific awakening of the nineteenth century, they were formulated in ignorance of the doctrine of evolution. This great principle of Darwin's has entered into and fertilised practically every sphere of modern activity; but its direct and intimate bearing on education has yet to be fully realised.

The education of the majority of children begins between their fifth and seventh birthdays, and continues for about ten 
years, after which they join the world as wage-earners. During the whole of this time they are kept closely to a study of books, and the ideal that is held before them is the acquisition of a maximum of book-learning. Facts are insisted upon as of prime importance, and the text-books which are crowded with them become the child's principal mental food. But isolated facts can be assimilated only by effort of pure memory, and therefore the cultivation of this faculty becomes the centre of the educational system. By learning heights of mountains, historical dates, populations, lists of proper names, exports, and exceptions to rules, the child's memory is hypertrophied out of all proportion. His other and no less important faculties must wither for lack of exercise, and his mind becomes distorted. With monotonous regularity come half-terminal, terminal, and annual examinations, when the absorptive power of the memory-sponge is estimated by methods which are not concerned with any of the other indications of the child's developing intelligence. By the results of this test, judgment is passed on the capacity of each child. Those unfortunates who by severe and unnatural effort have retained in their memories at the time of the examination the particular facts which are asked for, are deemed to have best acquitted themselves. They are rewarded with prizes, certificates, or scholarships which open the way to even severer feats of memory.

It is this system of examinations which necessarily sets the character to modern education. Apart from the fact that not even the broadest-minded examiner can, by any of the usual methods at his disposal, ascertain the real capacities of each individual on whose ability he proposes to decide, and that a retentive memory must always score to the disadvantage of mental powers of greater practical value, examinations infect the whole curriculum with a false intention, and lead to the minds of children being systematically "forced." The teacher is judged by the examination success of his class, and the school by its list of medals and distinctions gained in open competition. We cannot be surprised, therefore, that but little thought is given to those slow-growing but malleable processes of mind which are gradually converting the child into a man, and are 
destined to make or to spoil his adult life. The golden harvest which these qualities may bring ripens too late to interest his short-sighted instructors. They look for a speedy and tangible return for their efforts, and are impatient for a sight of the fruit, when they should merely be tending the opening leaves.

A sure test of the value of a system of education as a preparation for adult life may be found in the after-careers of those children who distinguished themselves at school, and in the school records of men who distinguish themselves in life. It will hardly be maintained that the greatest successes in life are the particular appanage of prize-winners and gold-medallists, or that the qualities that are specially serviceable in the examination-room are those that the universal experience of mankind has proved to be of greatest value. Yet in any right system of education the school must be essentially a preparation for adult life. It must set the same store on character, on practical knowledge, and on theoretical learning as will be given to them in the larger world. Further, school life must teach children not only how to work, but how to spend their leisure. No immediate return should be demanded, but the growth of the child's understanding should be gradually advanced in all its faculties. To attain these ends a teacher must not be a mere dealer in scholarship, a purveyor of facts, and a classroom disciplinarian. He must understand the human nature of children, and he must know how to direct it into wider channels than those of book-learning, and by other influences than those of authority.

\section{The Material.}

It would be worse than futile to elaborate any scheme of training that was not at every point and in every detail in natural accord with the biological proclivities of the human young. Early in his life a child passes the shallow gulf that marks off man from other mammals, but his subsequent existence is built up on the all-powerful animal instincts from which even the adult cannot be freed. They not only form an integral part of human life, but they are the predominating part of human life. Therefore, instead of attempting their repres- 
sion, we must recognise in them the only objective of education and the only possible roots from which can spring the finer qualities of mind that are the peculiar heritage of man. These primitive instincts must be studied at their bedrock origins, and the course of their natural development must be understood, if they are to be bent to the exigencies of social life. As has been stated earlier, the instincts of children are generally non-moral, and their impulses heed no ethical law; but nevertheless these same instincts and impulses form the basis of all future conduct, which, in most cases, will be good or bad largely according to the training.

Mental instruction must accord with the natural laws of nervous processes. Sensations, ideas, and associations of ideas are the tripod of intellect which is to support the higher qualities of mind. Now we know that the tendency of all nervous action is the formation of habits. In childhood this tendency is strong, and it is, educationally speaking, at once our greatest difficulty and our principal help. Mental activity, like muscular action, calls for effort, the amount of which diminishes with each repetition. Children, no less than adults, avoid any new mental exercise that demands this effort, and they prefer one which, by frequent practice, has become relatively easy. It therefore follows that the same stimulus, or series of stimuli, often repeated, tends to produce the same response, and a habit is formed which can be eradicated only with increasing difficulty the longer it has existed. A simple yet invaluable means is thus placed at our disposal by which we can inculcate good habits which will persist throughout life. Yet, on the other hand, the tendency to the formation of bad habits is even stronger. Habits cluster round lines of least resistance, and as education is an energetic attack on natural indolence, the majority of habits, by binding down the individual to his existing level, will impede and not facilitate progress. This is especially true of mental work. Children easily fall into habits of thinking and learning. They avoid, if possible, the exercise of their higher and more conative cerebral powers. They discover for themselves a short cut to learning, and they assimilate a lesson sufficiently well to satisfy 
a classroom interrogation and yet shirk the real educative value of the task, which is to call into play those mental efforts that are still exercised with difficulty. For this reason "spoonfeeding," which is so successful a method of imparting knowledge with a single eye to the examination pass-list, stupefies the best intellectual qualities, and reduces mental alertness and scepticism to a sheep-like level of docility and acquiescence. In every mental exercise we must make the child think for himself. He must examine each new experience in the light of past experiences, and must turn over and over in his mind every new fact or observation until he has appreciated its relation to his pre-existing knowledge. Only by this attentive, critical, even unbelieving attitude, will every fresh item of knowledge be inseparably welded into the growing whole that makes up the child's intelligence.

Yet the attentive mood is feeble in early life, and unless a child's interest is well aroused to the matter in hand, the mental impressions obtained from it will be transitory. Thus a lesson that is dull fails to awaken any interest, and a triple harm is done. Time is wasted, a repugnance for work is engendered, and the child is driven one step nearer to the acquisition of the most disastrous habit of inattention. Fortunately, all children are dominated by an outstanding weakness which we should endeavour to turn to our own ends. Children, while life is still fresh to them, are consumed with a curiosity which needs but little adroit fanning to burst into flame. Whenever we make a successful appeal to this foible, even the most indifferent child pricks his ears. In this way we can make a child provide his own gilding to our educational pill. Further, it is a wellknown fact that sensations are the most vividly perceived and are retained longest in the memory when the attention is on the alert for them. By enlisting a child's curiosity, therefore, we shall less often be compelled to go over work a second time, and this in its turn helps to keep away inattentiveness.

Enthusiasm is another feature of childhood which is largely the result of imperfect experience. By rousing the eagerness of children, we can be certain of their unflagging energy, and they will willingly undertake tasks from which they would turn 
in their cooler moods. This enthusiasm is another trait which we can use to good advantage. Even though it is by its nature only temporary, it may lead to permanent good, and will often open up side-roads of enquiry which will still be a source of pleasure long after the original enthusiasm has died away. These energetic moods, however, are not without their possible harmful results. There is always the risk of excess, and there is also the danger that subjects taken up eagerly for a time may be dropped again as soon as their novelty begins to pass. If this is allowed, it can only lead to unsustained effort and to lack of perseverance-qualities which are never strong in childhood, and need careful nursing. Children are capricious, and they heartily dislike being held to one occupation when their inclinations have passed to another; but this changeability of disposition, being natural to the age, should be treated with sympathetic consideration. We must accept moods and fitful tempers as part and parcel of youth, and must endeavour to bring them under control by gradual and persevering effort.

Lastly, we must remember that though children differ from adults in many respects, they share many of our weaknesses. Not the least important of these are sensitiveness to blame and desire for sympathy-two points which are only too often overlooked. Probably we have no more valuable test of an adult's capacity to handle children than his employment of blame and encouragement. A dread of reproof is commoner among children than is sometimes thought. Their eagerness to avoid blame may often be made more efficacious than punishment itself in keeping them up to standard. The longer some children are allowed to go without punishment of any sort, the stronger will be their determination to avoid all cause for punishment. The converse is no less true, and a child who is repeatedly punished soon grows callous. He comes to regard punishment as an unavoidable accompaniment to life, and its deterrent effect is almost completely lost.

Very many children have their lives made unhappy because their elders try to exact from them the same standard of morality as is expected from grown men and women. If parents no less than teachers would understand that children 
cannot possibly possess the sense of right and wrong that comes with adult years, there would be less injustice and much less unhappiness in the nursery and in the schoolroom.

\section{The Methods.}

We stand in need of a wider application of the principles of evolution to the educational methods of the present day. So far as mental processes are concerned, evolution has, of course, claimed the attention of educationalists before now, and Pestalozzi emphasised the necessity of making education conform in its order and in its methods to this development. A deliberate attempt, however, to impart an evolutionary or sociological character to a child's training can hardly be said to exist except in a very few cases. Even in these, the intimate mutual relationship between physical, moral, and intellectual training is not always understood.

If we are to place education on this broad basis, it is clear that we must adapt its methods to a far wider field than mere scholastic instruction, and that we must bring them into use at a much earlier period than is customary. The faculties of a child begin to develop and are amenable to training from the time of birth. Educative methods, therefore, should be introduced even into the nursery while the child is making the first efforts to use his senses, and the training should continue into adult years. The whole of this period may conveniently be divided into four stages, though we must recognise that each passes imperceptibly into the next, and that its duration varies with different children. During the first period, which begins with birth and may be said to last some two or three years, enormously rapid progress is made by children, though they have to rely almost exclusively on their own efforts in order to advance their understanding. They are but little responsive to methodical training, while discipline is applicable only in a rudimentary form. Although this first period is largely one of spontaneous education, we are able, as will be pointed out later, to render material guidance. From the beginning of the second period we can take a more active part in the training. The child's intelligence has become sufficiently advanced to 
respond to educational methods which leave nothing to chance influences, but tend directly to a fixed end. Our chief endeavour must be to teach obedience, which is destined to be the predominant motive for many years, until the child has become capable of judging for himself between right and wrong. Since the tendency of all children is to resist authority, the second period must extend over a number of years, until the principles of good conduct have become incorporated among the child's instincts.

When this most necessary advance has been made, we can gradually pass to the third period-that of adolescence. The power of independent judgment will now be making itself felt, and must be specially cultivated in order to take the place that a submission to discipline has filled in the past. We must appeal less and less to authority, but, instead, must encourage the child to develop his reasoning faculties. We must gradually accustom him to freedom and independence, and must encourage him to act on his own judgments.

The fourth and last period brings us to the stage of studentship. The training must now be specialised with a view to wage-earning. Authority must be withdrawn from sight, and the privileges of personal freedom granted. This period carries us beyond the limits of childhood, and it therefore requires only a few remarks here. Authority, though no longer seen, must still be in existence. It should be kept in the background, always ready, however, to make itself felt if required. It should be so used, that, while its existence is unknown to the better types of students, it should be a light hand on the shoulders of those who are mildly inclined to slack or to browse in forbidden pastures, but should keep a firm grip on those who are likely to be harmed and to harm others by their own inexperience or short-sightedness.

We must now consider the three first stages in detail :

The First Period-Spontaneous Education. - Throughout this period, children are relatively little amenable to direct educative training. They act in response to their natural inclinations, and therefore our opportunities for active direction are limited. Yet we should do wrong to leave an infant to his 
own devices, or to believe that nothing can be done to facilitate his mental development. In an earlier chapter we found that the essential change in his mind was an increasing acuteness of sensory perception, combined with an improved control of muscular movement. Sensorial training and motor training, then, are the two points to which we should direct our first efforts. In each instance we can provide the infant with suitable opportunities for the exercise of his powers; but we must leave him to avail himself of these opportunities.

In sensorial training, sight and touch require our special attention; hearing is of subsidiary importance, while taste and smell have but little influence. At first only fundamental differences between sensations are possible, and it will be useless to provide an infant with any object that is not essentially simple in every respect. In the case of sight, the earliest distinctions that can be drawn are those of bigness and smallness, of shape, and of brightness; later, moving and stationary objects must be distinguished. An infant can best be taught to make these distinctions for himself if we place two or three simple objects in his immediate neighbourhood, where they will always occupy a prominent position in his field of observation. Elaborate or multi-coloured toys can only confuse a mind that is still struggling to distinguish a cube from a sphere or a silvered globe from a rubber ball. Similarly, as regards tactile sensations, elementary differences must first be learnt, and to this end we must select objects that illustrate such qualities as roughness, smoothness, hardness, softness, warmth, and cold. Size and shape, though learnt partly by the eyes, will be further appreciated by the fingers. It is hardly necessary to add that all objects that are to be handled must be chemically and physically harmless, more especially as infants derive their tactile sensations largely from their lips and tongues. On the other hand, it is desirable that these objects should be small enough to be readily carried to the mouth, but not small enough to be swallowed.

At later stages a series of objects of increasing complexity will serve to train the senses to appreciate further differences of detail-such as distinctions between cotton, wool, velvet, and 
silk, between metal, wood, and cardboard, and between geometrical shapes. In arranging these exercises, we must be careful to avoid the danger of over-estimating a child's capacities. This would be a serious fault, because the simpler the object, the more strongly will it hold the child's attention, and the more instructive will its effect be on his mind.

With regard to the motor training, our chief aim should be to cultivate the power of accurate muscular movement. Co-ordination will be best developed by providing the infant with ample inducements to seize and to grasp. Not infrequently we see a nurse or a mother, whose infant is clutching the air in a vain attempt to grasp, move the desired object to the child's hand. Such interference is, of course, wrong. It produces the same educative harm as would result from setting a schoolboy a mathematical problem, and then providing him with the solution. In later months, young children can be taught more elaborate movements, such as rattling the contents of a box, striking objects together, opening lids, turning keys or screw-stoppers. Later still, they should be encouraged to build with wooden bricks.

The essential principle of infant education is to allow the child to amuse himself as he wishes, but to select the articles for his amusement with an eye to their educative value. He should be given ample time and freedom to "play," but a close eye must be kept on his actions. The exact stage he has reached will thus be known, and we shall be able to make preparations for the next steps. A warning must here be given against the almost universal nursery belief that the floor is the proper place for a baby's play. The floor is necessarily the least clean part of a room. It is trodden by boots that carry in the dirt from the streets. On it will settle the possibly infectious particles that are showered into the air in sneezing or in coughing; nothing could be better devised to entangle dust of every kind than carpets and rugs. Even fastidious parents, who would themselves decline to eat a piece of bread that had been dropped on the floor, will allow their child to sprawl and creep by the hour over the carpet. The little fingers are inevitably soiled, and then, from time to time, are sucked. Any article picked up 
from the floor will more than probably be carried to the lips. Everyone must have observed how infants, lying face downwards, delight to amuse themselves by sucking the cool linoleum beneath them. It should be realised by parents, nurses, and teachers that the floor is a favourite haunt for disease germs, and that the unavoidable draughts sweeping in beneath the door and across to the fireplace or to the window are well calculated to raise miniature dust-storms in the lower strata of air that the child is taking into his lungs. For these reasons it is very important that a clean rug or other covering should be spread on the floor to protect the infant. Better still is the plan of never using the floor as a playground. Instead, we should make use of a table, raised well above the draughts, and railed round to prevent any risk of an accident. An infant "pound" or "pen"- - a four-sided wooden railing or fence-can easily be made by any carpenter to fit a table, which can itself be covered by a waterproof mattress.

Although for many months an infant must be left to his own inclinations, no time is too early to begin the cultivation of regular habits of life. This important duty is often overlooked, or, what is worse, habits are allowed to develop which, a little later, will cause much trouble. Thus, mothers often accustom their babies to go to sleep at night by rocking their cradles or by singing to them. Useful as this soothing undoubtedly is, the child will sooner or later refuse to sleep without it. The mother is then compelled, night after night, to leave her other duties in order to quiet her child, who, under different training, might have been accustomed to fall asleep without any assistance. Regular habits in connection with the natural functions of the body are specially important. An infant who is given regular opportunities for relief, will not be long in forming cleanly habits ; whereas, by neglecting this point, we shall invite much distress and unpleasantness at a later age. Punishment is only too often inflicted in a useless attempt to cure a weakness for which the unhappy child is not to blame.

During the first period the infant will make his earliest attempts at speech. From the time when he is able to imitate words, we can do much to help his efforts. A summary of the 
stages by which a child learns to talk has already been given (p. 78), and this account sufficiently clearly indicates the steps we must take to increase his vocabulary.

Second Period-Disciplinary Training.-The second period, which continues throughout the greater number of years usually devoted to schooling, is not exceeded in importance by any other period. During this time the character of the child will, in the majority of instances, begin to assume its permanent shape, and therefore this second stage must be concerned principally with the cultivation and moulding of character.

Whether the child is at home or at school, obedience must be made the pivot of action. A great responsibility is thus laid on those in authority, who owe it to their charges and to themselves to act with consistency and without hasty judgment. We must on no account extort obedience by fear. We should avoid making any command if we do not intend to enforce it. Much moral damage is done to a child when he learns that orders can be rescinded, or that authority can be evaded by pleading or by specious excuses. A parent or a teacher who lightly enjoins an unnecessary or an unfair task, places himself in a difficult position, and harms the child, whether the order is persisted in or is withdrawn. Perhaps no error of judgment is more frequently met with in connection with children, than a display of authority which in the end is not carried through; and probably on no occasion is greater mischief done to a child's character, than on the day when he first realises that the authority which he had always regarded as a solid wall contains a wicket-gate which opens to artfulness or deceit.

But although authority is important, it is by no means the only influence to which children are susceptible. By appealing to their sense of fairness or kindness or to their affection, we can obtain a very strong hold over them. The system of prizes and punishments, which has been made the foundation of school discipline for generations, is gradually coming to be recognised as embodying a wrong principle. Apart from the fact that it directly appeals to motives of rivalry, which in their turn give rise to selfishness, it contains the error, exposed by Herbert Spencer, of encouraging children to expect a reward for good 
behaviour, and of visiting them with set punishments for their delinquencies and petty breaches of school discipline. Recently, a juster value has been set on prizes and punishments; but in schools much remains to be changed. ${ }^{I}$ Spencer particularly emphasised the necessity of allowing each action to bring its own natural reward or its own natural punishment, and he illustrated his point by the example of a child who leaves his wooden bricks strewn about the floor, instead of tidily packing them together. When next he wishes for the bricks, he must suffer the natural result of his untidiness, and go without. Again, it is better that a child who is unpunctual for a walk should be left at home, rather than that the brothers or sisters should be kept waiting, and that a set punishment should be inflicted on the dilatory child.

Yet another power that is invaluable in helping to form a child's character is instruction in the truths and ideals of religion. This should be given from an early period, but the same standard of conduct should not be placed before him as though he already had the experience of an adult. We should be content at first to lay the broad foundations of morality, and only gradually to impress an ideal on his mind, with the intention that when in later years he begins to think for himself and to form his own beliefs, he may be furnished with trustworthy guides. Up to this stage, the moral and ethical side of religious instruction should be given prominence over the doctrinal and theological. The important question therefore arises as to which of the two possible lines of moral instruction should be followed. Either the child must be taught that virtue will not develop in him unaided, but that he must draw his moral strength from God; or he must be taught that the seeds of good do actually lie within his own nature, and that it is within his own natural powers to make them germinate.

Whichever of these courses is adopted-and the decision must be made in accordance with the creed of the individual responsible for the child's upbringing-we must take particular

1 In the Ecole Alsatienne, in Paris, the whole system of rewards and punishments has been superseded by the personal influence of the teacher on the better side of the child. 
care to be consistent in our attitude. Either the child must become accustomed to refresh his moral understanding from without, or he must be taught to hold himself responsible for his thoughts and actions, whether good or bad.

If the teaching is to be on a religious, as opposed to an ethical basis, it is very important to remember that, compared with an adult, a child has but a small understanding of religious conceptions, and also that he finds greater difficulty in accepting religious beliefs that are contrary to his experiences in life. Every parent must have been called on by his children to answer questions which prove the existence of this difficulty, and show how little capable children are of comprehending theological beliefs-such, for example, as the omnipresence of the Deity-or even of distinguishing between the supernatural and the natural. On this account, and because instruction that cannot be appreciated is wasted, care should be taken not to force on children doctrines that must be meaningless to them for many years. Again, we should give practical recognition to the fact that religion owes much of its influence over adults to the appeal it makes to their emotional side. With the young, whose temperaments are immature, there is neither the same craving for religious comfort, nor the same susceptibility to its influence.

These considerations are enough to show that, like all other subjects dealt with in connection with training, religion must be taught to accord with the gradual evolution of the child's mind. Finally, it is desirable that instruction in the gloomier doctrines of theology should form no part of the teaching. Almost all children are quick to develop fears, and, what is more serious, they are often unable to prevent them overshadowing their whole lives. These fears play a larger part in child-life than is sometimes realised by those who have left their childhood far behind. By giving religious teaching which suggests or intensifies such fears, we are more likely to do harm than good.

Hand in hand with religion must go the moral influence of the beautiful. Unfortunately, we find that a systematic attempt is rarely made to develop the æsthetic sense of a 
child. The few exceptions are often those in which the parents, being themselves of specially artistic temperament, are apt to cultivate artistic qualities at the expense or even to the exclusion of others equally important. Æsthetics, however, should form an integral part of training, for without its help a child's development cannot fail to be incomplete and warped. The general lines on which this training should be given are clear. The asthetic sense is found in children even in their earliest years. Though in its beginnings it is, like other faculties, but little responsive to methods of active training, yet it should be directed into the right channels from the outset. It should be cultivated from two directions. On the one hand, we must provide the continuous, though passive influence of beautiful surroundings; on the other, we must take more active steps to develop an appreciation of what is best in form, in colour, and in sound. The influence of the surroundings should be as carefully attended to in the school as in the home, and not only should objects of artistic merit be placed about the child, but those which are likely to nullify their good effect should be rigorously excluded. With regard to the active training, we must seek the help of good models in painting, sculpture, music, and literature. At first the child will require to have an explanation of the elementary features which make these examples worthy of study. Later, he will be able to understand for himself the criteria which artistic work must satisfy. His taste will thus gradually form, and in time he will be able to know and to feel for himself the moral influence of the beautiful. This end will be attained with certainty only if he is encouraged to attempt productive work himself.

During the second period, attendance at school will occupy a large part of the child's time, and the mutual relationship between home and school must be understood. Our object should be to make the two environments complementary. The majority of children attend day-school only, and are therefore in constant touch with both influences; but often no intelligent effort is made to bring the two surroundings' work into harmony for the ultimate good of the child. Parents and teachers, 
by meeting together from time to time to exchange their observations on the child's progress and tendencies, can arrange for mutual assistance in eradicating faults or in developing habits of punctuality, order, and obedience. By this interchange of ideas, the teacher will become acquainted with any educational shortcomings in the parent, and the latter will learn the character and methods of the teacher. The classroom, we must remember, is a highly artificial environment, and enforces a habit of life which is foreign to children, to whom muscular restraint and sustained efforts of attention are unnatural. Moreover, day-schools still attach more importance to those qualities which facilitate the course of study and the maintenance of school discipline than to the development of the broader traits of character. They are chiefly concerned with a curriculum which lays down the amount of book knowledge a child must assimilate each term; and the teacher's business is to prevent any child from unduly falling behind at this work.

Just so far as the school mistakes its real aim, so far must the home fulfil the extra duty that is thrown upon it. Some parents consider that when the four-o'clock school-bell rings, education is in abeyance till the next day, and that, except perhaps for home-lessons, the child may be left to his own devices. Fortunately, other parents recognise that children must be controlled, directed, and helped by suggestion or example at home as well as at school, and that it is not enough to turn them out in the garden, the yard, or the street, to spend their hours aimlessly until bedtime. Children need to be taught how to make good use of their leisure. Half-holidays, Saturdays, and Sundays should not be allowed to pass without any improving work or exercise. Parental responsibility is not satisfied when a child has been ordered to "amuse himself." We cannot expect that children who are left to find occupation for themselves will develop good habits, and we must not blame them if they develop bad. Without supervision they will learn to associate leisure rather with indolence and tedium than with instruction and observation; or worse, to regard it as an opportunity for unimproving or vicious practices. Once 
this association has taken hold of a child, especially one of adolescent years, much difficulty will be experienced in teaching him the proper use of his leisure.

In boarding-schools, which are to a great extent both home and school, the necessity of supervising leisure no less than school work is generally recognised; but even in these cases the importance of filling every waking hour of a child's life, and of making each day regular and busy, is not always realised. A boarding-school possesses opportunities that a day-school lacks for imparting education in the widest and best sense of the word. It should be able to effect more good than a day-school and home combined. When children are always under the same control, and when this control is exercised by trained specialists who are free from parental indulgence and bias, a steady, consistent, and wise influence can be brought to bear.

If every home and every school were ideal, we should have no difficulty in finding an answer to the vexed question: At what age should we first submit a child to school environment? But the fact that either of these may be defective, makes it difficult to fix an invariable age-limit. In general terms, children should begin to attend school as soon as they are capable of deriving benefit from school teaching without suffering harm, bodily or mental, from their new surroundings or from their partial withdrawal from home influences. In our public elementary schools, instruction is given to children at an earlier age than in any other civilised country-namely, in their fifth year. At this early age they are unfitted for the strain and the risks of school life. For this reason it has been urged that the minimum age should be raised, and that school attendance should not begin until the sixth year at earliest. Some authorities, however, while admitting the possible harm of premature schooling, look at the home surroundings of the poor, and maintain that the dangers of running the streets and of playing outside public-houses is even greater. They believe that the earlier these children are brought under the influence of school, the better will be the result. The question resolves itself into a simple alternative. If a child is at home receiving no training at all, or only a bad training, and if the 
classroom training is fitted to his tender requirements, then school is the best place for him. But if the home is a careful one, or if the classroom atmosphere and the other points of an "infants' school" are not suited to a very young child, he will be better under his mother's care. With children of the middle and the upper social levels, where the home influences are usually better, the first attendance at school is best deferred until the seventh or even the eighth year.

Once school attendance has begun, the nature of the instruction given is of great importance. The irrational cult of memory, which constitutes the central rite of scholastic training, has already been discussed. Throughout the earlier years of school training, our aim should be, not the learning of cut-anddried knowledge from books, but the practical training of the child's senses, by means of personal experiences and experiments. At this stage, children do not require to supplement their first-hand observations of life by the second-hand records of those who have preceded them. They must be encouraged to make their own observations, to piece them together, and to form judgments in connection with them. In other words, they must be taught to teach themselves. There is never any difficulty in pursuing this method of training, since children are as pleased to use their own five wits as they are bored at the dreary labour of memorisation. Eyes and hands are to be specially trained, and the power of doing is to be set above the art of knowing. The practical and the concrete should take the place of the theoretical and the abstract. A liking for manual and industrial work characterises almost all children, and it is best that this work should be directed to fill the actual requirements of the school; children show much greater eagerness if they are making articles which they know will be put to practical use in the school. Similarly, their fondness for collecting and for object-lessons should be turned to good account. Nature-study provides us with a most valuable opportunity, not only for cultivating their powers of observation, but for teaching them to appreciate the great law of cause and effect. Yet another benefit afforded by this study is a right understanding of man's place in the animal world. 
A relatively small amount of bookwork is necessary until later. Sufficient intellectual exercise will be provided by learning to read and to write, and by studying the rudiments of arithmetic, with, perhaps, some acquaintance, conversational and not grammatical, with a foreign language. We must not as yet look for studious habits. We must recognise and accept the preference which children of this age show for the more recreative items in their training. These subjects can easily be made more instructive than ordinary school lessons. By giving them this prominence, we shall succeed in teaching children to use their hands, their eyes, and their ears to better purpose than they would if they were kept at their desks.

Third Period-Adolescence.-Our earliest attempts to substitute reason for authority, as the actuating motive in education, can generally be made some years before the period of adolescence. As the guiding principles of conduct begin to crystallise from experience, children come to understand the reasons that justify or forbid particular actions. Once they have reached this level, we can appeal with increasing frequency to their conscience rather than to their obedience. Unless discipline is thus loosened, self-respect and the finer ethical appreciations can have but limited opportunity to grow, while individuality is unable to develop except under the stimulus of freedom and responsibility.

We should be particularly careful to deal with adolescents in a sympathetic manner. Though they understand their oncoming maturity only imperfectly and lack confidence in themselves, they nevertheless resent being treated either as children or as mental inferiors. This age proves itself a fertile soil for the growth of ideas of altruism, and of community of action and organisation. Young people are gratified when first they realise that they are members of a community, and they take pleasure in finding that they have responsibilities to others. The moral effect of cultivating this attitude is of the greatest service in developing those qualities which, later, will make up citizenship and all its social obligations.

With regard to the work suitable to this period, physical exercise must still be given an important place, inasmuch as 
growth is far from complete. Workshop training should occupy a prominent position in each day's programme. The character of the mental training should now undergo change. The powers of observation must still be actively cultivated, but they should be supplemented by a greater amount of study. The reasoning faculties require developing. We shall find scope for them in the various branches of mathematics, and in the different forms of science, especially in experimental physics and chemistry. About this time we shall find that children have formed some notions of political and state organisations, and will profit by a study of the salient facts of social history and progress. Now, no less than before, we must be careful to prevent the formation of routine methods of learning. A want of mental thoroughness is a not uncommon failing at this period, and children learn the art of displaying their little knowledge in a manner to suggest that it has been understood, although, in point of fact, it has merely been committed to memory. If this habit is allowed to continue, it will undo the whole intention of education, and will lead to perfunctory and bad methods of study.

At every age children are peculiarly unresponsive to teaching that is given on synthetic lines. They have but little faculty for inductive reasoning, and are repelled by isolated facts and by disconnected observations. On the other hand, they are keenly appreciative of analytical methods, where the whole is first presented to their understanding, and is then dissected stage by stage into its component factors. Their interest follows this deductive process without difficulty, as it accords with the methods of observation with which they have been familiar from infancy. The learning of lists and all such mental exercises of pure memory are even less suited to this than to an earlier age. Such efforts not only occupy time, which might be devoted to better ends, but produce wrong ideas of the importance of memory. These ideas usually cling to a child for some years after leaving school, until experience has shown that what can be obtained with absolute accuracy from books of reference is mere lumber in the insecure chambers of memory. Until the methods of examination already referred to are 
superseded, "cramming" with facts and with figures is not likely to be abolished, and the fairer and more scientific methods of ascertaining a child's capacities-as by testing his manual dexterity, his powers of verbal and literary expression, his observation, and his resourcefulness-will continue to find but little employment.

If we have been careful to accustom children throughout their earlier training to regular and well-filled days, they are not likely at the outset of adolescence to revert to lazy habits. It is particularly important that they should have already formed the habit of employing their leisure to good purpose, because with the advent of puberty a dangerous epoch is entered. New sensations and new desires are experienced for the first time, and children are unable to understand the change through which they are passing, or indeed in many cases to realise that a radical change is in progress. Their dispositions are profoundly disturbed. They grow shy, awkward, or self-conscious, and become moody and uncertain in their temperaments. They develop habits of introspection, subjecting their actions and their motives to self-analysis. They contrast themselves with others to their own disadvantage, and on this account they become depressed or lose confidence in themselves.

At this time they stand in special need of guidance to dispel their unfounded apprehensions and to ward off the bad habits, mental and physical, that tend to establish themselves. Until recently it has been held that we should keep the knowledge of the true and simple facts of puberty from boys as well as from girls, and that by ignoring the most important physiological development in life we shall be serving the best interests of a child. Fortunately, the prudery of this attitude, by which children are deprived of wise counsel at the most critical period of their lives, is at last becoming realised. Those who understand children best, and are most solicitous for their welfare, recognise the great necessity of instructing them in a matter which is no more impure than the mind chooses to make it. By explaining to boys at the proper season, and in the proper manner, the biological facts of generation, we cannot possibly produce harm, but, on the contrary, will do away with an in- 
calculable amount of mental and physical suffering and injury. If we withhold this instruction, boys will obtain their knowledge -and they inevitably must obtain it-from the less desirable of their companions. They will not only imbibe harm in this way, but will get the pernicious idea that the whole matter is one for secrecy, to be kept out of the sight of their elders. This fact alone will endow the subject with an unnecessary and undesirable importance, and, as has been conclusively shown, will, a few years later, provide a profitable income to the innumerable quacks who trade on the juvenile ignorance of sexual physiology.

An objection has been raised by some that, though in theory children should be made acquainted with these facts, yet in practice the instruction is difficult to impart. It is, indeed, inadvisable to provide children with a direct explanation of these phenomena in connection with themselves-such a course being likely to foster rather than to prevent habits of introspection-but we have a means ready to hand in the teaching of biology. The principal facts of reproduction in respect to plants and to animals can be readily explained, and the young people can be safely left to apply this knowledge to themselves.

\section{The Special Training of Girls.}

The necessity of providing for girls an educational training comparable in its thoroughness to that required by boys, has aroused interest only in recent years. Though we no longer believe that girls have been equipped for adult life when they have acquired a smattering of those accomplishments which, imperfectly mastered, make for superficiality, we have yet to free ourselves from the trammels of a tradition which has ignored the special requirements of the sex. Much progress must be made and much lethargy dissipated before girls can obtain an education suited to their special capacities and to their special function in the world.

At the present day some people demand a similar training for girls and for boys. The profound physiological differences, however, between men and women, and the consequent dis- 
similarity between their mental and temperamental outlooks, are conclusive proof that strictly identical lines of training are wrong. On the other hand, the sexes possess very many qualities in common, and these can be cultivated in the same manner. Indeed, in the earliest years of life there are, from an educational point of view, no material differences between girls and boys. Later, though long before adolescence has been reached, the two paths begin to diverge, and finally, with the advent of the secondary sexual characters, a wide separation is effected, still bridged, however, by the many points of resemblance that have persisted from the first. From the point of view of co-education, three stages can therefore be conveniently recognised. First, a stage when identical training is applicable to both sexes. Next, a stage which is marked by the earlier indications of divergent development. Finally, a stage in which both sexes possess much in common, but each presents important differences.

In the first stage, girls and boys will profit equally by the same training, and there is no object in separating them. In the next stage, girls no less than boys require to be trained by discipline, and must be taught the virtue of obedience. The elementary habits of tidiness, cleanliness, punctuality, and order, are as important, and selfishness and bad-temper as undesirable in one sex as in the other. Both are responsive to the same moral influences. Their mental processes run on identical lines, and they can be taught to observe, to think, and to act by the same methods. On the other hand, the power of physical endurance and of muscular activity is, even in early years, appreciably less in girls than in boys, and the difference becomes more marked with time. Girls are more tolerant of authority, and more ready to accept without demur what is set before them. They are more sensitive to blame and to commendation, more painstaking, and more satisfied by detail. The deepest reaching difference of all is that girls are influenced by sentiment, while boys prefer to rely on their intelligence.

Are these differences too profound for the successful coeducation of the sexes? They are for the most part merely differences in degree, and are therefore responsive to the same 
methods of training; while the more emotional characteristics of girls are no bar to their common instruction with boys. So far as the points in common are concerned, they comprise practically all that is involved in moral training and in purely class-room training. The mutual influence of the two sexes would be of the greatest benefit by imparting a vigour of mind and of enterprise to the one, and a gentler and less assertive tone to the other. To secure this mutual effect, we must be careful to avoid giving a numerical preponderance to either sex, and we must also arrange that the girls and boys who are trained together are of similar ages. In adolescence, when, perhaps, a more serious danger might be apprehended, experience has shown that fears on this score are unfounded. Co-education, though only in its beginnings in this country, has been widely tested in the United States, especially in high schools, and has proved itself successful. The responsibility for the satisfactory working of the arrangement rests with the girls, and they are not found wanting.

Yet even when this system is adopted, we should be wrong to assume that no real difference is to be made between adolescent girls and boys. The end for which boys must be trained is inot the goal of the other sex. Women have their own responsibilities and their own duties, and to these their training must be adapted. It is the fortune of most girls in later life to undertake the upbringing of children, and this duty implies the correlative responsibility of controlling a home. If a woman is to fulfil these functions, she must above all things possess physical health, while book-knowledge, though by no means without value, is of subsidiary importance. ${ }^{1}$

With regard to the first named, girls even more than boys must be taught the rules and practice of healthy living. Yet all too commonly they are lamentably ignorant of the requirements of health. The exigencies of sex accustom the feminine

1 It is not without interest to contrast this view, which is being increasingly widely held at the present day, with the opinion expressed by Steele two hundred years ago. "The management," he says, "of a young lady's person is not to be overlooked, but the erudition of her mind is much more to be regarded." 
mind to sensations of ill-health, and, as a very frequent result, women become inattentive to those precautions and observances on which common prudence should insist. They are often unwise in matters of diet and hours of sleep. Numbers of them do not understand the necessity of regularity in their daily lives. The natural functions are neglected in girlhood, and the permanent habits of constipation that usually result become responsible for much of the ill-health of young women. Similarly, many girls set but little value on the good effect of open-air exercise.

At certain times girls are not in possession of their normal health, and are not fitted to undertake their ordinary tasks. On these occasions they require to be treated.with special consideration, and, what is more, to treat themselves with special consideration. This necessity should be impressed on them from the time when it first arises. Again, many girls are intentionally kept in ignorance of the real nature of phenomena which cannot fail to occupy a considerable share of their attention. The common result is that, brooding on these things, they become anxious and worried, until in countless instances they fall into morbid and neurotic states of mind. If, as we have maintained, suitable instruction in kindred subjects is necessary for boys, some counsel and enlightenment is surely needed by girls, whose lives are dominated by these matters.

Considering next the scholastic education of girls, we must recognise that girls, in later life, are not permanently employed in the same occupations as men. Book-learning-still less bookishness-should not be the object of their training, and an extensive knowledge of the subjects that figure in the average school curriculum is not likely to be of practical value in the majority of cases. The only excuse for inducing a girl of ordinary ability to devote herself to special study in any of these subjects is, that she may fill her time and have some definite interest in life. But if a girl is rationally trained-that is to say, undergoes a training that brings her natural inclinations into line with her natural function in life-she would not find her interests unattached. Therefore any such special study would be merely an unsatisfactory makeshift to cover the defects 
in her general training. If, of course, a girl reveals some special aptitude for any particular subject, her case is different. It is right that she should be afforded every facility to develop her talent. It is, however, wrong to urge a girl to a severe or exhausting course of study with the sole object of obtaining for her some examination distinction. By undertakings such as these, she is required to live a wholly unnatural life for weeks or months together; her health suffers, and in the end she may have memorised an indigestible mass of facts without having absorbed the underlying principles, which alone are of educative value.

But though the average classroom syllabus is so little adapted to girls, there are two large subjects in which they should all be thoroughly trained. These are housekeeping and child-rearing. These two, interpreted in the broadest sense, include, with religion, the best interests and the highest aspirations of women.

Far from being an uninteresting subject, domestic economy -cooking, dressmaking, laundry-work, household management, including the pecuniary side of housekeeping and marketingis, if properly taught, truly educative in its effect, and can engage and satisfy the interests of every average girl. Any girl who has received a school training in which these subjects have taken a leading part, is well equipped for her future work, and has acquired habits of method and thoroughness that cannot fail to be of service to her during the interval between leaving school and becoming married - that is to say, during the time when, in most instances, she will need to work for her living.

The other subject of cardinal importance in training - though it has never yet been recognised as such in practice-is the science of child-rearing. If the reader needs evidence that the average mother is appallingly ignorant of the elementary methods of child-culture, he will find it in full in the later chapters of this book. Yet the amount of preventable harm wrought by mothers on their children is not to be measured by the heaviness of infant mortality alone, but, in addition, by the innumerable though unrecorded instances of impaired health among those who survive. Girls, though destined to be mothers 
and to be responsible for the lives of helpless children, are permitted-nay, encouraged-to grow up in absolutely complete ignorance of these vitally essential duties. It is no exaggeration to say that a single generation of mothers intelligently alive to their responsibilities would effect a greater revolution in the condition of the race than half-a-dozen centuries of political reform. Child-study, when made a subject of training, should be interpreted widely, and must be taught in practice as well as in theory. We must give teaching, not:only in the physiological necessities of the young, the steps in their mental development, and the preservation of their health from the common dangers, but also in the facts of heredity and the broader aspects of eugenics that bear on the subject.

Before concluding this section, we must say a word on the personal influence of the teacher on the pupil. This influence may be very great in respect of girls, since they are naturally inclined to accept and to copy whatever is presented to them as authoritative. The character and the experience of the teacher therefore counts for much. Yet if we ask what is the type of teacher chosen at the present day to hold up the mirror of womanhood to a younger generation, we shall obtain an answer that is by no means satisfactory. School mistresses have been compelled, by years of exacting study, to follow a life which is not the natural life of a woman. Their professional work, taking the place of marriage, has necessarily shut them out from the consummation of a woman's life. They are chosen on a plan which definitely excludes those who have had experience in the very matters which are of foremost importance in a girl's education. The absurdity of the arrangement will, perhaps, be better realised if we look for a moment at the qualities we seek in a master for our boys. We endeavour to place them under the control of a man of matured character, who has had long experience of men and things, who knows the world as men know it, and who is able to fill his school with an atmosphere that is manly in the best sense. Yet we are at no pains to obtain corresponding advantages for our girls. How often does a parent select a school for his girls because its headmistress has been a shrewd observer of the world as women 
know it, and whose views of life have been widened and mellowed by her experience as a wife and as a mother? Does he not look for a school where the teaching staff possess university degrees, hold examination honours, and are reputed to be successful teachers of piano-playing and modern languages ? $\mathrm{He}$ accepts these qualifications, and shuts his eyes to the narrowness of thought and dryness of spirit which seem inseparable from so many girls' schools. Perhaps the day will come when we shall recognise the advantages of placing a girl's training under the superintendence of those who, in rearing their own families and in managing their own homes, have learnt the highest lessons of womanhood. Then, and not till then, will the influence of the girls' school approximate to the ideal, and a new and liberal influence will be at work in modelling the feminine character on the fine lines along which it naturally tends to develop.

\section{Abnormal Types of Children.}

We must now briefly mention certain abnormal types of children who, without being pathological, stand in need of a somewhat special upbringing. Between normal and morally insane children there lies an undefined borderland where, owing either to perverted instincts or to defective self-control, social obligations can be taught only with difficulty. These children have rightly received much attention in recent years, and public opinion has followed the medical belief that they are to be considered in a class by themselves, and must not be judged by everyday standards. Ordinary methods of education must be modified before being applied to them, and if we wish to eradicate their defects we must recognise their nature and origin early in life.

Of the different types of these children the commonest are the selfish, the passionate, the cruel, the lying, the precocious, and the neurotic.

Extreme selfishness is specially frequent in the offspring of one-child marriages, where the parents regard their child rather as a pet to be humoured than as an individual requiring careful training. More serious than selfishness is passionateness, though 
this defect often tends to disappear, and many violent children develop in time docile dispositions. Lying is a specially grave vice in children, for they are quick to realise that they can practise it without discovery, and frequently benefit by it. We must carefully distinguish this deliberate assumption of the habit of lying from the want of truthfulness, or rather of precision, which is venial in early life. Thus many children will describe incidents which are real enough in their own imaginations, but possess no foundation in fact. Other children are frightened into untruthfulness by the fear of an over-stern master or father. Others, again, are untruthful merely because the incongruity between the actual facts and their own statement of these facts appeals to their sense of the grotesque. Each of these forms of untruthfulness stands, of course, on an entirely different footing from that of the lie deliberate. Thieving and cruelty are to be regarded as specially grave offences. If allowed to continue unchecked, they may lead in later life to criminal acts. Children who are subject to these vices are sometimes cured of them only with much difficulty.

In each of these above types we require to devote special attention to the particular defect. A close supervision, kindly but firm, is essential. Punishment for specific offences is, as a rule, out of place, and we must depend on a general moral influence exerted patiently over a considerable time.

Precocious children require special care in their education, lest by being pushed on too rapidly, they suffer in health or in mind. It is, no doubt, a temptation to a teacher who finds an exceptionally receptive and intelligent child among his pupils to urge him to extra study. The parents themselves are often only too ready to lend their influence in the same direction. Yet the health of precocious children is at any rate no less, and may be more, precious than that of children of average intellect. These children are likely to suffer in the long run if an unnaturally heavy strain is thrown on their developing brains, or if any one of their faculties is cultivated at the expense of the others.

Probably the largest group that requires mention here is that of neurotic children. Neurosis in early life is usually, if 
not always, an indication of a defective upbringing. To what extent schools themselves are responsible for its production is not easy to gauge, though probably it is not great. The conditions which tend to induce this morbid state-such as excessive sympathy, idleness, frequent notice of the child's own feelings and introspection-are not as a rule characteristic of school life. Yet, on the other hand, morbid states of mind are apt to arise in school children who are overworked or go in frequent dread of punishment. Sometimes the apprehensions incidental to examinations is the starting-point. In other cases acute disappointment at being kept behind in a class when others have been moved to a higher has been sufficient to make children neurotic. The saddest and worst result of this mental unbalance is seen in the not infrequent records of children who, finding the anxieties of school too heavy for them, have been impelled to take their own lives. These suicides, which are most numerous in the most highly civilised countries, can often be directly traced to an over-rigid or unsympathetic school discipline. In Prussia, these tragedies are particularly frequent, and average no fewer than fifty-four each year, more than a third of which are due to the abovementioned motives.

Children who are inclined to neurotic manifestations usually pay much attention to their own feelings, and therefore special efforts should be made to turn their thoughts in other directions. By keeping their minds occupied, and by filling their lives with interests outside themselves, we shall be able to afford them better opportunities for developing a robuster temperament. We must not treat them with any lack of sympathy, because, if anything, they need even more consideration than healthy children. We should not, however, allow them to realise that they stand in need of special care, but on the contrary should impress on them their resemblances to other children. 


\section{CHAPTER IX}

\section{IDIOTS AND FEEBLE-MINDED CHILDREN}

Methods of Public Control: Historical; Guardians of the Poor ; Metropolitan Asylums Board; Special Schools; Results of Training in Special Schools; Recommendations of the Royal Commission on the Care and Control of the Feeble-minded. Number of MENTaLly Defectives in England and Wales. Causes of Mental Deficiency. Classification of Mental Deficiency: Idiots, Imbeciles, and Feeble-minded; Congenital Idiocy; Microcephalic; Hydrocephalic; Mongo!s; Cretins; Primary Amaurotic; Genetous ; Birth Palsies; Acquired Idiocy; Epileptic; Post-Febrile ; Traumatic ; By Deprivation; General Remarks on Idiots. DIAGNosis of IDIOCY in INFANTS. TRAINING OF IDIOTS AND MENTALly DEFicient ChILDREN : At Home and in Institutions; Combined Defects ; Results of Training ; General Care ; Moral Training ; Physical Training; Sensory; Muscular; Speech; Intellectual Training. MORAL INSANITY.

\section{Methods of Public Control.}

THE claims that idiots possess to the protection of the community are only now receiving full attention. Hitherto they have been looked on as creatures deserving little more than to be left to their fatuousness. It is not difficult to understand how even the benevolent have been repelled by the want of humanness of an idiot child, who lacks most if not all the qualities that raise man above beast. That the law denied him civil rights was not the heaviest of his afflictions, and there are probably country people still living who can remember the time when the village idiot was chained like a felon in the common pound, an outcast and a butt for scoffs and blows.

But while the idiot in poor surroundings has for centuries endured this unhappy lot, his better circumstanced brother has 
had every care taken of him. History provides an interesting explanation of this distinction. Towards the end of the thirteenth century, Edward I., influenced by a Scottish practice by which a feudal lord established a wardship over an idiot's lands, laid claim to the control of the property of all natural fools. In spite of opposition from the nobles, he succeeded in securing this right to himself. Edward II. made the privilege sure by the help of the Statute of Prerogatives, and from this time onward the greatest attention has been paid to the propertied idiot. The welfare of the unpropertied idiot, however, has been nobody's concern, although, strictly speaking, the sovereign, as pater patriæ, exercises jurisdiction over all insane persons. In 1660, when the Court of Wards and Liveries was abolished, the king's powers over the estates of natural fools were delegated to the lord chancellor, and since then idiots have remained in his care. The expenses incidental to this guardianship have always been so great that the poorer idiots were left to the care of their friends, or, in a few instances, of religious charities. In the sixteenth century some of these mentally deficients were to be found in poorhouses, then being established for the first time. Others were kept in gaol as destitute or dangerous persons, but the majority still went unprovided for. Two hundred years later the law dealt with them on the specific ground that they were a danger to the community. They were to be apprehended by the churchwardens or other parish authorities, and kept locked up, or, if necessary, chained up. Not until the end of the eighteenth century do we find the first recognition of the claims of the insane to human treatment, when, in 1774, an Act was passed "for regulating madhouses" by means of government inspection. In the middle of the nineteenth century a notable advance was made by the opening of the first asylum specially reserved for idiots.

The arrangements in force at the present day may be briefly described. Mentally deficient children can either be taken from home and placed under the care of some public authority, or they can remain at home, and, if the facilities exist, can receive education at one of the special county schools. 
All mentally defectives are eligible for poor-law relief, whether indoor or outdoor. This relief is controlled by the guardians of the poor, who are authorised to follow various courses. For example, they may admit cases into a workhouse; or they may send them to suitable schools, to idiot establishments, or to asylums; or they may make grants of money, food, or clothing.

The workhouses provide insufficient accommodation for the number of mentally defectives, and for various reasons they make an unsatisfactory environment for the upbringing of a feeble-minded child. Their inmates are at liberty to leave when they are sixteen-at the very time when they are most likely to fall into evil habits. The frequent result is that numbers of them, released from supervision, become wastrels or criminals, while many of the girls take to immoral courses. This official powerlessness to detain uncertified mentally feeble persons against their will exists in all institutions which receive them.

In London, mentally defectives are dealt with by other authorities besides the guardians. The Metropolitan Asylums Board maintains seven small residential homes and an industrial colony at Darenth for nearly 2000 inmates. The same Board and the London County Council each provides asylum accommodation for those cases that are certifiable under the Lunacy and Idiots Acts.

As the insufficiency of the foregoing arrangements became realised, a move was made to provide centres for the education of the great number of children whose deficiency was of a relatively mild type. Since 1899 , school authorities have been empowered, under the Elementary Education (Defective and Epileptic Children) Act, to provide special classes for children who, "not being imbecile, or merely mentally dull, are, by reason of mental or physical defect, incapable of receiving proper benefit from the instruction in the ordinary public elementary schools, but are not incapable, by reason of such defect, of receiving benefit from instruction in such special classes or schools." At the present time, some I 40 special county schools have been established in England and Wales, 79 being in London. They are very unevenly distributed 
throughout the country, and all of them are in urban centres, usually where there is a large industrial population. No school fees can be charged-except for the expenses of conveying children to and from school-and parents are compelled to send their mentally deficient children between their seventh and sixteenth years. Seeing that the proportion of defective children on the school registers is 0.79 per cent. of the total register, we can understand how inadequately 140 schools can deal with this large number.

The children for whom these schools are specially provided are those with slight degrees of mental deficiency. The tendency, however, has been to fill the classes with an increasing proportion of the severer types. As a result, many children are received who are fitted only for custodial homes and are never likely to be able to earn their own livings. It has been shown that in the London special schools, while from 5 to Io per cent. of the children were found sufficiently intelligent to be returned to ordinary schools, and 45 per cent. could do manual work well, and could support themselves wholly or to a material extent, no fewer than 50 per cent. were capable only of working under supervision. ${ }^{1}$ The following table ${ }^{2}$ shows the

\begin{tabular}{|c|c|c|c|c|c|}
\hline \multicolumn{3}{|c|}{ Economic Condition. } & \multicolumn{3}{|c|}{ Moral Condition. } \\
\hline $\begin{array}{l}\text { 1. Not earning at all } \\
\text { 2. Occasional small earn- } \\
\text { ings } \\
\text { 3. At fairly regular un- } \\
\text { skilled work . } \\
\text { 4. Earning good wages } \\
\text { 5. Supported by husband }\end{array}$ & $\begin{array}{r}16 \\
8 \\
26 \\
7 \\
1\end{array}$ & $\begin{array}{r}27 \cdot 6 \\
13 \cdot 8 \\
44 \cdot 8 \\
12 \cdot 1 \\
1 \cdot 7\end{array}$ & $\begin{array}{l}\text { I. Satisfactory or harm- } \\
\text { less } \\
\text { 2. Unsatisfactory : } \\
\text { 3. Doubtful } \\
\text { 4. Acknowledged as im- } \\
\text { becile : } \\
\text { 5. No record: }\end{array}$ & $\begin{array}{r}20 \\
13 \\
7 \\
4 \\
4 \\
14\end{array}$ & $\begin{array}{r}34.48 \\
22.40 \\
12.06 \\
6.94 \\
24.12\end{array}$ \\
\hline & 58 & $100 \cdot 0$ & & 58 & $100 \cdot 00$ \\
\hline
\end{tabular}

results of thirteen years' work at a special school in South London. During this time, 58 children left school over age, and their economic and moral capacities are set out in the two columns. Among the I2'I per cent. earning good wages there

1 Mrs Dickinson Berry, M.D. $\quad 2$ Prepared by Mrs Owen Fleming. 
were several who were merely dull, and were not really feebleminded. The 86.2 per cent. under the first three headings really stand very close together as regards their mental capacities, and most of them would probably have been better under custodial treatment.

These results show clearly that, in addition to special schools, other educational arrangements are necessary for the feebleminded. It is useless to keep in these schools children who are not likely to improve; while, on the other hand, the merely mentally dull should not be treated as mentally deficient. Probably the part played by these schools will undergo a change in the near future, and the direction which this change is likely to follow will be indicated below.

A complete alteration in the conditions affecting the care of our feeble-minded population is proposed in the exhaustive Report of the recent Royal Commission on the Care and Control of the Feeble-minded (1908). After going in great detail into the above questions, the Commissioners expressed decided opinions on the unsatisfactory and inadequate nature of the present-day arrangements. Their conclusions are too extensive to be even mentioned here, but their principal recommendations may be summarised. Their scheme is, briefly, as follows :-

The entire care of all mentally deficient persons is to be placed under a central Board of Control. Subject to its approval, each County Council or County Borough Council, acting through a "Committee for the Care of the Feebleminded," will make provision for its own mentally deficients. The Committee will have power to make what arrangements seem best for all such persons under 2 I years whose home surroundings are unsuitable. After this age, these persons shall be reported upon to the Board of Control, who shall decide what further steps are required. In cases where the home environment is satisfactory, the Committee will endeavour to make a voluntary arrangement with the parents for the appointment of friendly visitors to watch over the well-being and training of the children. Weak-minded persons under 21 may, upon a doctor's certificate and without the intervention of any judicial authority, be placed by their parents or by the 
Committee in registered institutions for the mentally deficient. They may be retained in these homes with the consent of the Board after they have reached the age of $2 \mathrm{I}$. If, however, the Committee thinks it desirable, they may be transferred to the care of a relative or friend. Special provision is made that no parent receiving help from the Committee is to be deprived of any right-as, for example, the franchise. On the other hand, he shall be liable to contribute an agreed weekly sum towards the cost of maintenance of his child.

After suggesting that receiving houses, where cases can be kept under observation and temporary treatment, should be provided not only for the poor, but for paying inmates, the Report recommends radical changes with regard to the training of mentally deficients. The Committee is to undertake the complete training of all those children not otherwise suitably provided for. All special schools already established are to pass from the authority of the Board of Education to that of the Board of Control, and contracts for mentally deficient children who have been boarded out, are to be transferred in like manner. Additional special schools, classes, or homes may be established, and a child may be removed from one to another as the Committee thinks necessary in the child's interests. In those cases where there is doubt whether children are deficient or only dull, they may be placed on a special probationary list and kept under observation until the point can be settled.

The object of these schools, as they are at present conducted, will undergo considerable modification. Instead of being the centre of a system of special education, they will be merely incidental to a larger organisation of industrial and institutional training. They will serve two purposes. Primarily, they will be used to train those children who, in later life, will be able to look after themselves or to do useful work under supervision. Their second function will be to act as observation centres. Children whose degree of weak-mindedness cannot be exactly appraised will be kept under observation at these schools until enough has been learnt of them to justify a forecast of their future. Doubtful border-line cases will thus be recognised. They will be found either not to require special 
training or, at the other extreme, to be incapable of profiting by the training. In either case they will be transferred to more appropriate surroundings, and much labour, thought, and money will be saved.

The Report recommends special measures for those mentally deficients who are criminal. Their general purport is to prevent a feeble-minded prisoner from being judged by normal standards, and to place him, not in prison, but under the control of the Committee. Even should he be acquitted of the offence charged against him, the Court will have power to enquire into his mental condition with a view to making provision for his care.

In brief, the principle adopted by the Royal Commission is, that persons who, owing to mental defects, cannot take their part in the struggle of life, should be afforded by the State such special protection as is suited to their needs. The real claim of these people to State help is their mental condition, not their poverty or their crime, and this help should be continued in each case so long as it is necessary for the individual's good.

The workhouses should, as far as possible, cease to be asylums for the chronic mentally deficients. "Intermediate" hospitals or inexpensive homes should be erected to serve this purpose. "Family care" or "guardianship" is another cheap method of providing for these cases. In its general lines, this latter system is widely accepted in many Continental countries. Thus, at Gheel, a village some twenty-five miles from Antwerp, 2000 patients are received in the village or in surrounding farms, mostly as boarders. A medical director, five medical assistants, a pathologist, and lay inspectors supervise the system, and every house is visited once a week. Very similar methods are in force in France, at Dun-sur-Auron and at Ainy-le-Château. Here the arrangement is a central colony, with an asylum, where patients are boarded in families, and a number of out-lying sub-colonies. In some parts of Scotland, family guardianship has been adopted as a kind of local industry, and works smoothly and satisfactorily. Yet another method of the greatest service is the system of large farm colonies. These have been tried with success in Canada and in the United States. 
The Number of Mentally Deficients in England

\section{AND WALES.}

The percentage of feeble-minded people in England and Wales has never been exactly determined. In the census returns before 190 I the three words "idiot," "imbecile," and "lunatic" were treated as synonymous with "insane," and the numbers collected under each of these headings were added together and published as the number of "insane" people. In I90I, the words "feeble-minded" were substituted for "idiot." The total number of "insane" in this census was more than twenty per cent. larger than in IS9I (1 32,654 to 97,383). It was surmised that this increase was largely made up of "feebleminded" persons who had not previously been notified. The most exact estimate is that recently made by the Royal Commission. The mentally deficients, other than certified lunatics, were enumerated in certain selected typical areas with a population of $2,362,222$, and from the figure thus obtained, the number for the whole population of England and Wales $(35,527,843)$ was estimated. The result was a total of 149,628 , or 0.46 per cent. of the population. As the following table shows, the majority of these are under the control of public authorities, and only 45,488 are cared for privately :-

Estimated Number of Mentally Defective Persons, excluding Certified Lunatics, in the General Population of England and Wales.

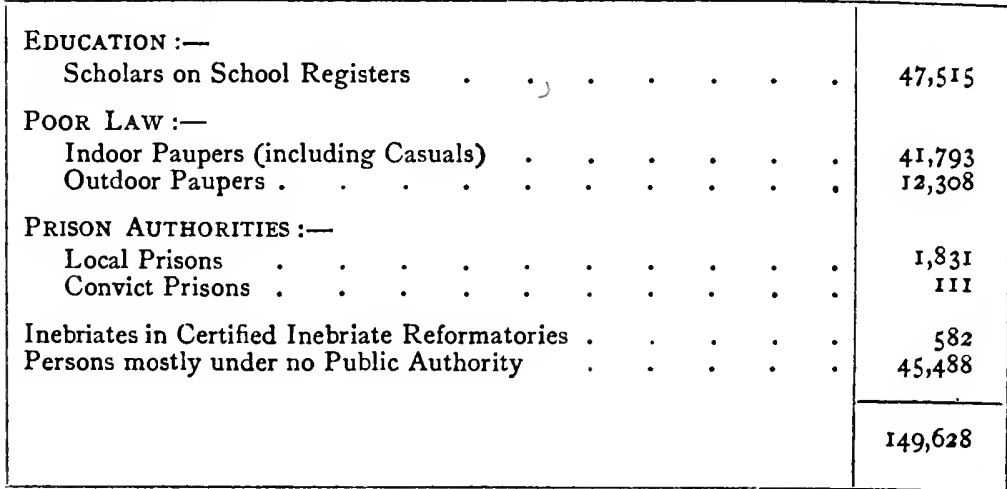




\section{The Cause of Mental Deficiency in Children.}

As to the causes of mental deficiency, we have but little really trustworthy information to guide us. Alcohol, tubercle, syphilis, and consanguinity have been invoked as provocative causes, but a factor with stronger claims than these is heredity. The birth of feeble-minded children in successive generations of a family has been recorded many times, and indeed idiocy has been regarded as the most frequently propagated of all mental derangements. Haller, in his Elementa Physiologica, ${ }^{1}$ mentions two young ladies, who, though idiots, had their defect sufficiently counterbalanced by large dowers to enable them to marry. For a century their taint spread through several families, and some of their descendants of the fourth and even of the fifth generation were idiots. Most of the witnesses before the Royal Commission agreed that a very large proportion of mentally defectives are the offspring of mentally defective parents, or are members of families in which other nearly related members are mentally deficient. Though this opinion is supported by much evidence, we must not accept it as definitely proved. Many believe that environment exercises a far more important influence. This we must in part allow, when we recall how little qualified mentally deficient parents are to train their children. In many of these cases the young are familiarised from infancy with the evils of drink, crime, and sexual vice. They are physically weakened by neglect or ill-treatment, and before long they show unmistakable signs of mental degeneration. Heredity and environment, however, are not the only factors, for a story not uncommonly heard from a respectable and healthy mother of a defective child is that her family and her husband's are free from the taint as far as their history goes, and that the parents themselves have already many children, none of whom is below average intelligence or physique. The collected observations still leave the subject in much obscurity, but one point of special interest stands out from the others. It has

${ }^{1}$ Quoted by Ireland, The Mental Affections of Children, 1898. 
been estimated that male idiots are more than twice as numerous as female idiots. ${ }^{1}$

\section{Classification of Mental Deficiency.}

The classification of mental deficiency is not merely of theoretical interest, but bears closely on questions of treatment and training. Its varieties are more or less strictly delimited pathologically, and a familiarity with their points of difference is essential to everyone who is called on to advise in individual cases. By a preliminary and convenient subdivision, all children who are mentally deficient from birth or from an early age are separated into three groups-idiots, imbeciles, and feebleminded. Idiots are the lowest grade, and are unable to guard themselves from the common physical dangers of daily life. On this account they cannot with safety be left alone. Next are the imbeciles, who, though capable of this elementary intelligence, are not able to earn their own living. The feebleminded are the highest grade. They can earn their living, but cannot compete on equal terms with their fellows, and are without ordinary prudence in the management of themselves and their affairs.

No sharp line can be drawn between these three grades. There would be no great difficulty in discovering every intervening gradation between a somewhat dull child and a jibbering idiot with unclean habits and fewer instincts than the lowest of mammals. The absence of a boundary line between one group and the next is no drawback to the classification, but, on the contrary, is useful from the educational standpoint. It shows that the differences are merely of degree, and that in any given case it may be possible to train the child some steps up the ladder towards sanity.

Another system of classification has been adopted by those who regard the subject from a social or administrative point of

${ }^{1}$ This relationship is suggestive when considered in connection with the well-known fact that the heads of new-born boys are larger than the heads of new-born girls, and that they must therefore meet with severer pressure during delivery. 
view. This system divides all mentally defectives, excluding lunatics and the subjects of senile decay, into seven classes: (I) idiots; (2) imbeciles; (3) feeble-minded; (4) moral imbeciles; (5) epileptics who are also mentally deficient; (6) inebriates who are also mentally deficient; and (7) deaf and dumb or blind who are also mentally deficient. Moral imbeciles are persons who from an early age display some mental defect coupled with strong vicious or criminal propensities on which punishment has but little effect. The term does not include those children who are the subjects of moral insanity and yet show no other mental defect. These children will be dealt with specially at the end of this chapter.

A more scientific classification than either of the above is one founded on a clinical and pathological basis. In the first place, two main classes must be recognised-the congenital and the acquired. If we exclude headless and brainless monsters, it is perhaps open to question whether an infant at birth can, strictly speaking, be mentally deficient. As we saw in Chapter III., the average new-born baby possesses an intelligence that does not rise above simple reflex action, and therefore no signs of mental deficiency may be obvious even to a skilled observer, until weeks or months later. Congenital idiocy, therefore, should be taken to apply to the intellect that fails to expand in a normal manner after birth as a result of some cause acting before or during birth. The cause is congenital; the idiocy appears later. The acquired types of idiocy result when the further development of a previously healthy mind is arrested by disease or injury.

Each of these two main classes comprises different types, the principal of which are included in the following list :-

CONGENITAL IDIOCY :-

I. Microcephalic.

2. Hydrocephalic (macrocephalic).

3. Mongolian (talmuc).

4. Cretinous.

5. Primary amaurotic.

6. Genetous.

7. Birth palsies. 


\section{ACQUIRED IDIOCY :- \\ I. Epileptic. \\ 2. Post-febrile. \\ 3. Traumatic. \\ 4. By deprivation.}

In addition to the above, scrofulous, syphilitic, emotional, neurotic, and toxic forms have been described. We need, however, concern ourselves only with the distinguishing points of the above eleven more important varieties.

The Microcephalic Idiot is recognised by the size and shape of his head. Its circumference is usually less than 19 inches, often less than 18 inches, and may be considerably smaller. The occipital portion is but slightly developed, and the back of the head is often in line with the nape of the neck. The narrow sloping forehead recedes to meet the occiput at a pointed vertex. The mental capacity of these cases depends largely on the size of the brain, and therefore this point must be considered both in estimating the probable improvement in intelligence and in planning the details of the education. The outlook is favourably influenced if the power of speech is present, but many microcephalics are incapable even of the most rudimentary articulation. Though we must not anticipate great progress in any of these cases, many of them can be trained to simple, industrial work, such as gardening or farming. Microcephalics are not specially liable to premature death, and many of them live to old age.

The Hydrocephalic Idiot possesses a skull that is too large both for its face and its body, and sometimes the weight of the head is more than the child can support. It bulges in every direction, and all its diameters are increased. The veins on the temples are often big, and the anterior fontanelle may be tense and prominent as the result of the increased pressure within the cranium. The pathological change is, in most cases, a collection of fluid in the ventricles of the brain (hydrocephalus internus), but occasionally the effusion lies between the brain and its membranes (hydrocephalus externus). In the latter and rarer condition the head is not necessarily very large, and it may actually be smaller than the average. 
In the first year or so of life, cases of internal hydrocephalus might, if the head is only slightly enlarged, be confused with other enlargements, as, for example, from rickets. The distinction, however, should be made without difficulty. The condition of the fontanelle alone is often enough to prevent error. ${ }^{1}$ Hydrocephalics vary much in their mental powers, but most of them respond satisfactorily to education. In temperament they are, as a rule, docile and passive. Their lives are not necessarily short, even in marked cases.

The face of the Mongol proclaims the type of idiocy at a glance. Its almond-shaped eyes are obliquely set, and they seem widely separated owing to the well-developed folds of skin that bridge across their inner corners. A curious Chinese appearance is thus given to the face, and the grotesqueness is completed by a bright complexion, a mere button-shaped knob for a nose, and a coarsely-marked tongue which hangs outside the mouth so that the child is constantly slavering. Mongols vary in intelligence. Most of them are capable of distinct improvement, especially as they are much given to mimicry, and commonly show more than the rudiments of affection for those who tend them. They can usually be trained for some simple industrial work, but education beyond this level may be wasted. They are delicate in constitution, and their peculiar susceptibility to cold usually puts an end to their lives before puberty. Phthisis is responsible for most of these deaths.

A Cretin is probably the only idiot that could be confused with a Mongol, but no one who has seen an example of each is likely to make the mistake. In cretins there is a striking arrest of physical as well as of mental development. However long they live without medical treatment-ten, twenty, thirty years-they are often no bigger than children of three or four years. Their bodies are fat, with the large pendulous bellies of the middle-aged, and they often have double chins. Their skin is dry, harsh, and yellow. Their hair seems prematurely sparse. Their hands are broad and their fingers are stunted. Though their eyes are normal in shape, there is often a pig-like look about them, as they are half-closed by puffy eyelids. The 
nose is flat and the under-lip is coarse, perhaps because the child sits open-mouthed, lolling out its large tongue. Cretins are singularly unemotional, and Darwin states that, unlike other idiots, they do not weep. ${ }^{1}$ Their movements are slow and almost ponderous. Thus a cretinous boy passed his time gazing blankly before him for several minutes together, then with a deliberate blink he turned his head slowly to stare again into space. He understood simple instructions, but executed them only after long pauses. If asked to give his hand, he made no response for some four or five seconds. Then without further request he slowly and laboriously extended his arm; but almost before the action had been completed, his mind lapsed, and he was again blinking into the air.

Cretinism stands apart from all other forms of idiocy, inasmuch as its immediate cause is known and is remediable. The condition is due to the deficient function of the thyroid gland, and may be cured by administering the extract of the thyroid glands of animals. If the treatment is to be fully successful, it must begin in the earliest years of life. Under these circumstances, a cretinous idiot may have his intelligence completely or almost completely restored to him. Indeed, he may even become bright-witted. A typical cretin came to the outpatients' department at the Evelina Hospital some three or four years ago. Since his recovery, he is frequently to be seen playing in the neighbouring streets, and I have noticed that he generally assumes the leadership among his comrades.

The next type of idiocy, though rare, is one of special interest. Primary Amaurotic Idiocy (also called Infantile Cerebral Degeneration or Amaurotic Genetous Idiocy) is the name of a condition first described by Sachs of New York. It occurs almost exclusively among the children of Jews, and the initial symptoms appear about the third month after birth. Mental development is arrested, and the power of muscular co-ordination necessary for balancing the head and supporting the trunk is lost. Blindness and muscular rigidity follow, the reflexes are exaggerated, and the progressive degeneration ends in death within the second year.

${ }^{1}$ Expression of the Emotions, 1872, p. 156. 
The Genetous type includes most of those idiots whose condition is attributable to causes acting before birth, but whose characteristics do not bring them under any of the above headings. There is no apparent cause for their mental failure, of which their features may show little or no indication. Many of them are pleasant looking or even prepossessing. Often, however, we can notice, on closer examination, some suspicious feature, or perhaps the face as a whole may be of a "low" type. A sloping or asymmetrical forehead, mis-shapen ears, eyes of an unusual appearance, squint, nystagmus, and a high-arched palate are not uncommon defects. These children may be spiteful and passionate, but under patient training they may become affectionate and docile. Genetous idiots are peculiarly susceptible to tuberculous infection, and according to $\mathrm{Dr}$ Ireland, two-thirds of them die from phthisis.

Birth-Palsy.-The last variety of congenital idiocy is that resulting from injury at birth. In cases of this nature there is no question of any error of development or of hereditary taint. These children are well-formed-sometimes it is their very size that brings them their misfortune-but owing to severe or prolonged labour, often associated with partial asphyxia, their brains are injured by external pressure, or by the rupture of a blood-vessel. Muscular paralysis-paraplegia or spastic diplegia-results, and the children become mentally deficient. When the motor injury is of the nature of a hemiplegia, the intellect is less severely affected-to the extent of weak-mindedness only-and we may be able to find little amiss except that the emotional side is controlled with difficulty. The outlook for many of the paralysed cases is less unsatisfactory than would be thought when first they come under observation. Improvement, though, of course, not cure, is the rule. The progress is measured by the severity of the brain injury, and for this reason spastic diplegics respond less to training than do paraplegics or hemiplegics. Many of the more serious cases develop convulsions, which may easily play further havoc with their already feeble intelligence.

The first of the acquired forms of mental deficiency is Epileptic Idiocy. Just as in adults loss of mental power may 
follow epilepsy, so, in children, the intelligence may be repressed from the same cause. In the latter the degeneration is not necessarily a profound degree of idiocy, but often stops at imbecility or even at feeble-mindedness, and, of course, epilepsy may co-exist with normal intelligence. We may say that, as a rule, the defectiveness of the mind is more marked the earlier in life the epileptic attacks begin and the more frequently and persistently they recur. In milder cases the mind often becomes appreciably brighter if the attacks are separated by fairly long intervals. This recovery is only temporary, and with renewed seizures the brain is again dulled. Progress is irregular and uncertain, and despite every attention many cases go from bad to worse. Further, epileptic idiots, though often dull or drowsy for some time after an outburst of fits, are for the most part irritable and less amenable to training than other idiots. Thus of 80 epileptics in the Earlswood Idiot Asylum, Dr Shuttleworth found that 55 per cent. were incapable of improvement, as compared with 6 per cent. of the other inmates.

Traumatic Acquired Idiocy is most nearly allied to the idiocy from birth-palsies, but the term is restricted to cases arising subsequent to birth-cases in which the mental development has been normal up to the time of the injury. Falls on the headas from a perambulator-may be the starting-point, and the immediate cause is usually a depressed fracture of the skull, a thickening of a cranial bone or a contusion of the brain. The degree to which the intelligence is impaired depends on the nature of the injury. Recovery, though slow, may, in some cases, be almost, if not quite complete.

Post-febrile Idiocy is, as the name implies, the result of one of the specific fevers, and it occurs when the acute disease has been complicated by cerebral embolism or thrombosis. Under this heading we must also place the instances of idiocy in children who have survived attacks of post-basal meningitis, or of acute encephalitis. Post-febrile cases are by no means hopeless, and they may improve very considerably.

The last variety that we shall describe is Idiocy by Deprivation. In this type the central nervous system is not at fault, but the sensory channels of sight and hearing have been destroyed, 
and the brain, shut up in darkness from the outside world, cannot develop. Examples of this terrible misfortune are happily rare, and are likely to become rarer, owing to the fact that some of the cases have resulted from the neglected treatment of the specific fevers, especially scarlatina. A worldfamous example of the condition is Laura Bridgman, who, when six years old, was found by $\mathrm{Dr}$ Howe of Boston as a blind, deaf-mute child with an imperfect sense of smell. He undertook her education, and began his teaching by making her associate two particular manual signs with two simple articles placed in her hand-namely, a pen and a pin. When at last she perceived the connection between the signs and the objects, the way was opened to further instruction, and she learnt in time to understand all the manual signs of the alphabet, and later to identify the letters in embossed type. Thus she acquired the power of reading and finally of writing. The next great difficulty was to enable her to appreciate abstract and moral qualities. This was overcome by teaching her to associate friendly and unfriendly acquaintances with the tastes of sweet and of sour fruits respectively. Her regular education was continued until she was twenty years old, by which time she had acquired a large vocabulary, could converse readily with all acquainted with the deaf-mute signs, and kept a diary of her thoughts and experiences. She lived to reach her sixtieth year, and died in $1889 .^{1}$

Of idiots in general, we may say that their sensorial imperfections are not, as a rule, marked. Their sight and their sense of touch are sufficiently good, but their hearing-especially in the case of cretins-may be dull or even congenitally absent. Their sensibility to pain is sometimes remarkably obtuse, and they will endure sores or injuries with unconcern. Instances are known in which bodily wounds have been self-inflicted with no more apparent motive than to pass the time. Their powers of attention are at a low ebb, and they may take little or no

${ }^{1}$ Dr Howe: Forty-third Annual Report of the Perkins Institute and Massachusetts Asylum for the Blind. Quoted by Ireland, Mental Affections of Children. 
notice of their surroundings. In harmony with this fact we commonly find that their artificially aroused interest is quickly fatigued. They have but little control over their emotional side. They may be irritable, passionate, spiteful, delighting in harm and destruction, but more often they are easy-going and contented. Some weak-minded children are strangely fascinated by fire, and to gratify their desires will commit acts of incendiarism.

With regard to the general prognosis, we shall find that on the whole the congenital idiot is a more hopeful subject for training than the acquired idiot. The idiot whose appearance betrays his nature is more easily improved in mind than his fellow whose features might pass for normal. This distinction, which is contrary to popular ideas, has been concisely summed up in the statement that the outlook varies inversely with the comeliness of the child. The more distant future is uncertain. Many succumb to phthisis before reaching adult years.

Treatment is, as a rule, limited to educational methods, and these we shall consider below. Special medicinal remedies are serviceable only with cretins and with epileptics. With the former the thyroid extract must be continued throughout life. Epileptic idiots may respond to treatment by bromides in so far as their fits are concerned, but satisfactory results are the exception, and in many cases the attacks will come and go irrespective of the treatment.

Surgical treatment is of limited use. Operations on the cranium have been performed for microcephaly and for hydrocephaly. In the latter condition the intention has been to drain the collection of fluid. In the former the assumption has been made that the growth of the brain had been stopped by a prematurely ossified skull. More probably the cause and effect in this explanation should be interchanged, and certainly the results of these operations have not been of a satisfactory nature. A more promising field for surgery is offered by idiocy from birth-palsies, from traumatism or from epilepsy. In these cases the removal of bone or of meningeal bloodclot might reasonably be expected to lead to a renewed development of the brain. 


\section{DIAGNOSIS OF IDIOCY IN INFANTS.}

The diagnosis of idiocy in a child of some few years can be made, naturally enough, without difficulty, and with a knowledge of the types of the affliction, we can decide what particular example is under observation. In the earliest months of life, on the other hand, mental deficiency is less apparent, inasmuch as what we may call mental sufficiency is still elementary. An idiot baby of a couple of months may be capable of performing all the acts normal to its age, and only at a later date, when its life is becoming more complicated, will it betray its defect. Yet even at this stage parents often fail to notice what is obvious to a skilled observer. The earliest possible diagnosis of the condition is desirable, if only for the satisfaction of the parents, of whom some are nervous and fearful, reading weakmindedness into the normal actions of their child. The sooner their apprehensions are dispelled, the better for their peace of mind. Other parents at the opposite extreme regard their geese as swans, and cannot even suspect a blemish in their child; and others again who, though unable to blind themselves to their misfortune, hope against hope, refusing to admit what all the world can see.

The key to the early detection of idiocy is an intimate knowledge of the psychology of normal infants. If we know that babies of so many months should do such and such things, but should not do such and such other things, we have a standard by which our patient can be compared. The infant idiot is revealed by its acts of omission as well as by its acts of commission. Sometimes it is preternaturally good, never crying and never discontented. Often, however, it is particularly restless. Perhaps the two earliest signs we should look for are a lack of interest in its own surroundings, and an imperfect power of grasping with its hands. A third sign which, like the second, is an expression of incomplete co-ordination, is the inability to balance the head on the shoulders. This control is not normally acquired until the third month, and therefore its absence is of little significance before this time. Curiously enough, a mother does not often notice these peculiarities, and 
her first suspicion may not be roused until the time when her infant should begin to sit up. She then notices that on putting the child into a sitting position, its shoulders become hunched, its back is rounded, and it topples over. A symptom that is very commonly offered as the reason for seeking medical advice is, therefore, a "weak back." To this is often added lolling of the head, for this defect will now have become more noticeable. Fortunately a weak back very much more often means rickets than idiocy, and the diagnosis must not be based on this one symptom, but confirmatory signs must be sought. The appearance of the face may help, and we should carefully study its varying expression. Sometimes we notice that the face is peculiarly expressionless, but that from time to time the features are broken by a fatuous smile. Often we observe that the mouth hangs open, so that the child is constantly slobbering. In Mongols, of course, the almond-shaped eyes are distinctive. The size of the head may be suspicious, but more often we observe some abnormality in its shape or symmetry. Additional help may be found if we notice one or more of the "stigmata" of idiocy. These include a number of physical blemishes. A narrow, high-arched palate, ears that are strangely modelled, epicanthus, squinting, nystagmus, deformed heart or feet, abdominal hernix, and undescended testicles, are among the most frequent stigmata. Most, if not all, of these bodily defects are of not uncommon occurrence in children of sound intelligence, and therefore their value in diagnosis is restricted to corroboration of a conclusion already suggested by other evidence.

With somewhat older children we may learn that teething is backward and irregular, that the power of walking, like that of sitting, is much delayed, or that articulate speech has not been attempted.

No less important than the physical examination of suspected idiots is the quiet observation of their behaviour when they are left to themselves. Many idiots are extraordinarily restless, but their movements are for the most part purposeless and monotonous, and they may accompany their actions with a neverending chatter. When somewhat older, idiot children will respond to a trivial annoyance with an outburst of uncontrolled 
passion. Finally, the stage is reached when their mental and moral shortcomings are patent to all.

\section{The Training of IDIOT and Mentally DEFICIENT CHILDREN.}

The modern, humane treatment of idiots dates back but little more than half-a-century, to the time when Séguin, a largehearted schoolmaster, undertook the training of the inmates of the Bicêtre in Paris. $\mathrm{He}$ based his educational methods on the principles of physiology and psychology, and he applied them directly to the mental and physical deficiencies of his charges. By the regular exercise of their muscles and organs of sense, he attempted to cultivate their limited powers, and to waken their dormant intelligence. This system placed the management of idiots on a scientific foundation. A new era began, and at the present time few civilised countries are without special institutions in which Séguin's methods are in daily practice. $^{1}$

Though the principles of training are the same in all children, we must be careful to arrange the practical details to meet the necessities of each case. We find, for example, that, while some idiots are easily improved, others are untrainable, and the distinction can be made only by experiment. With the lowest grades every attempt at training will fail; whereas the child whose intelligence is merely feeble may eventually be able to take his place among normal citizens. The question of employment, however, is, and has always been, a difficult one, as a glance at the etymology of the word will show. Idiotes, the term from which our word is derived, was applied by the Greeks to anyone not holding public office-that is to say, to any private individual. Later, the meaning became modified to express not the individual, but the incompetency that excluded him from official duties. Finally, its sense became further narrowed to

1 Traitement Moral, Hygiène et Education des Idiots et des Autres Enfants arrièrés dans leur développement, par Edouard Séguin: Paris, 1846. A full review of this book may be found in the British and Foreign Medical Review, July, I 847 . 
denote those who by reason of mental deficiency were incapable of employment.

In order to prevent too sanguine an anticipation, it is well to realise from the beginning that improvement and not cure is the limit. "Once feeble-minded, always feeble-minded" is the rule; and many children will require to be placed under permanent custodianship. The question therefore arises: What is the best form of supervision? If the mother can herself assume active and full responsibility without neglecting her other domestic claims, it is permissible and in most cases advisable for the child to be tended at home. A mother can prove herself second to none in carrying on the training. An infinitude of patience is required, and gentleness, sympathy, and forbearance must never be wanting. Of all guardians, the mother is most likely to possess these virtues, for often it is she alone who gives affection to her afflicted child. Yet maternal qualities are an inconstant factor, and not every mother will make a good custodian. The neurotic, the feeble-minded, or the vicious woman should not be entrusted with the responsibility, nor should the quick-tempered or the impatient. There are mothers who conceive an active and intense aversion from their idiot children. Others, impelled only by sense of duty, will strive for years, until, exhausted by incessant worry, their selfcontrol weakens, and they find difficulty in showing the necessary forbearance. For their own sakes, as well as for the sake of their children, they should be counselled to transfer their charges to other hands. More than once I have known a mother who, after having been unsparing in solicitous care for an imbecile child, grew in the end disheartened, for the reason that she could obtain no spark of affection from her child to compensate her for her anxiety. In the case of a mentally defective boy, a day is likely to be reached when the mother is physically incapable of restraining his turbulent actions.

The best plan, then, from the point of view of the child's welfare, is, undoubtedly, that the guardianship should be carried out at home. This is especially desirable if, in addition, the efforts of the parents are guided and encouraged by the occasional supervision of some specially qualified visitor, whether 
medical or not. This arrangement will answer satisfactorily in the majority of cases. But where it is adopted, the medical man must be prepared to advise as to the educational methods appropriate to the case, to explain their significance to the mother, and to supervise their application. He should from time to time take a general survey of all the conditions in connection with his case, including not only the progress of the child, but the health and well-being of the mother and of the other members of the household. If any of these are unsatisfactory, he must be prepared to recommend different arrangements, advising always for the good of the greatest number. In every case in which the home influence is bad, the child for its own sake should be transferred to a better environment. Among the poor, where feeble-minded children often have feeble-minded parents, the domestic surroundings are sometimes of the worst. Vice and cruelty prevail, and the helpless idiot child is neglected if not actively ill-treated.

The choice of an alternative arrangement must be largely influenced by the social position of the parents. With the well-to-do, the services of a specially trained attendant may be preferred, but in many cases a better plan is to place the child in a private home. There are many charitable institutions where paying patients are received, and there are a few private establishments for idiots, registered under the Idiots Act. Other private homes, not so registered, are for feebleminded cases only.

With the poor, one of the courses mentioned earlier in the chapter must be followed-namely, attendance at a special school for mentally defectives, admission to a special home, to a workhouse, or, if the case is certifiable, to an asylum. In the somewhat uncommon instances of children who suffer from some defect in addition to their mental deficiency, difficulty in placing them is likely to arise. Four varieties of these "combined defects" are recognised. Some mentally deficient children are blind, others are deaf-mutes, others are epileptic, and others are cripples. The blind and the deaf form only small groups. Of the former there are only about 160 examples in the whole of England and Wales; the latter are somewhat more numerous 
and in London alone they number some 50 to 60 . The cripples are a large group, owing to the number of mentally defectives who are paralysed. Each of these classes requires very special arrangements for its care. On the one hand, they cannot be taught with children who, though blind, deaf, or paralysed, are mentally sound; on the other hand, they do not respond satisfactorily to the methods employed for ordinary mentally defectives. Whatever is done for them, but little improvement is likely to be effected. Indeed, one authority ${ }^{1}$ maintains that their training is not worth its expense. At present the only institution in the country for blind and mentally deficient children is Stormont House, under the control of the London County Council. The same authority has established, at Homerton, the only institution for deaf mentally defectives, but its accommodation is insufficient for the needs of London alone. The epileptic mentally defectives are even worse off, for there is no institution that deals with them. ${ }^{2}$ The idiot asylums refuse admission to epileptics, the epileptic colonies decline to accept idiots. With regard to the paralysed mentally defectives, a first step on their behalf is promised by the London County Council, who have decided to undertake their teaching at schools for the mentally defective, whither they can be taken by ambulance.

Though these children with combined defects are not promising subjects from an educational point of view, every ordinary mentally defective child, whether kept at home or sent into an institution, should be systematically trained. The earlier this is undertaken, the greater is the prospect of success. Before the details are decided on in any individual case, an estimate should be made of the child's mental, physical, and moral capacities. As a help towards this, the following table will be useful. It is, with slight modifications, one given by Dr Barr, of the Elwyn School of Feeble-minded Children in Pennsylvania. ${ }^{3}$

${ }^{1}$ Dr Tredgold.

2 The Ewell Epileptic Colony, established by the London County Council, provides accommodation for over 250 men and nearly 60 women, who are insane epileptics.

${ }^{3}$ New York Medical Journal, I903, p. I I57, June 27 th. 


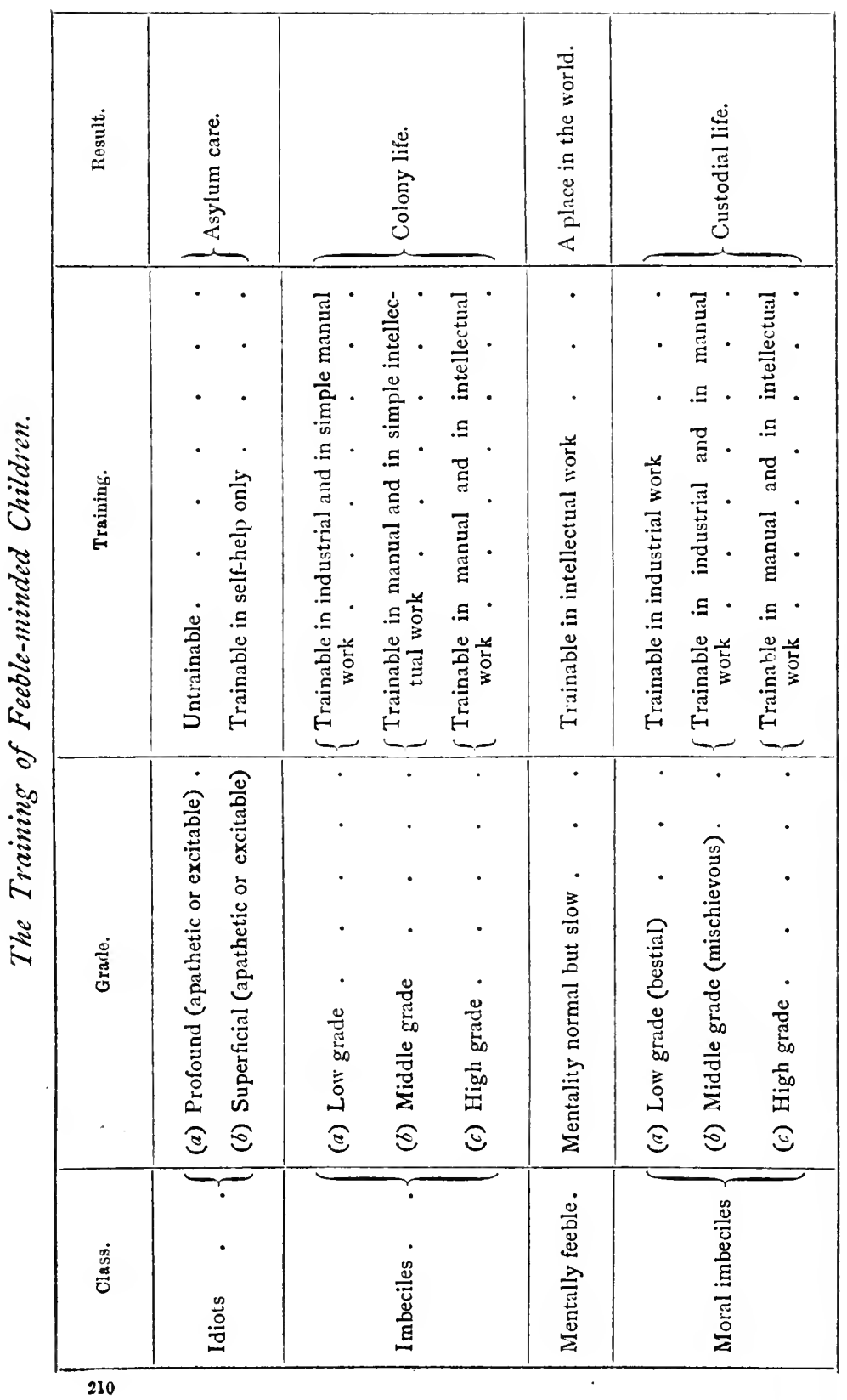


This division is, of course, purely one of convenience. As has already been pointed out, there is an unbroken chain from the apathetic idiot to the dull child. The classification is, however, of help, because it shows in simple form the kind of improvement and the career that may be anticipated in any case.

The educational care falls under four headings-general, moral, physical, and intellectual.

The General Care comprises all those means required to maintain a child in a good state of health. Personal cleanliness can often be secured only by constant attention. Some idiots are unable to feed themselves, and at meals they need assistance. Their clothing must be warm, to discount the feebleness of circulation that is often associated with idiocy. Their bodies and limbs should be well protected, in order to prevent injury, whether accidental or self-inflicted. Exercise is no less beneficial to these than to other children, and from the first they should be given opportunities of using their muscles. We have already dealt with the special medicinal treatment of cretins and epileptic idiots.

The Moral Training is by no means always as satisfactory as might appear at the outset. Months and even years of persistent effort may be required to produce a return. Our first attempt should be directed towards the inculcation of good habits with regard to the natural functions. Prompt opportunities for the regular relief of the rectum and bladder are essential. Children who possess only imperfect control in this direction must not be punished, but rather should be encouraged by judicious rewards to acquire better habits. The point next in importance is, perhaps, the cultivation of restraint over such emotions as those of passion, spite, and the desire of injuring and destroying. Herein lies a task that is anything but easy, and may be impossible. Obedience and respect for authority are essential, if training is to continue; but we must foster these qualities, not by physical force, but by word, gesture, and expression. Patience and persuasion must replace coercion. It may be possible to substitute truthfulness and a sense of responsibility or honour for the converse vices, but even an 
elementary appreciation of these abstract virtues is beyond the understanding of many mentally defectives.

Later in life-at the time of puberty or earlier-the habit of masturbation frequently develops and is often ineradicable. A more serious moral question arises in connection with criminal acts committed either by or against those who are mentally feeble. The former, though frequently conceived in a cunning and brutal way, are not common, because most imbeciles are harmless, even when their natures are irritable. The most important of the latter are sexual offences against imbecile girls and young women. The only means by which we can prevent these iniquitous acts is to keep female mentally defectives under close supervision. ${ }^{1}$

The Physical Education includes: (a) the training of the several senses; (b) the training of the muscles to perform regular, controlled, and co-ordinated movements; (c) the sensori-motor training required to develop the power of speech. Practically all mentally deficient children, with the exception of those afflicted with the profoundest idiocy, possess a sufficient development of their nervous systems to provide a foundation for some improvement, however slight. With most of them a fair aptitude for industrial or manual work can be cultivated. It is necessary to remember that the goal towards which we should direct our educational efforts is not the fashioning of a normal child, but the manufacture of as serviceable an

1 The proportion of mentally deficient women who have illegitimate children is high. Thus in a series of 165 such women, nearly two-thirds of their children were illegitimate. In another series, twenty-two women in two small poorhouses had eighty-eight illegitimate children. This evil is accounted for by two reasons in especial. Feeble-minded women cannot be detained in a workhouse or elsewhere against their will. They leave when they wish, and once they are free from supervision they are without the strength of will to protect themselves from outside dangers. The second reason is that by the Criminal Law Amendment Act, 1885, the difficult onus of proving that a male offender knew the woman was an "idiot" or an "imbecile" is thrown on the prosecution, while the law does not protect a woman who is merely feeble-minded, and is neither idiotic nor imbecile. Further, any offender, provided he is not an asylum or workhouse attendant or officer, is permitted to set up the defence that the woman was a consenting party. 
article as the defective material at our command will allow. By bearing this limitation in mind, we shall not waste our time or our energy in attempting the impossible. Rudimentary faculties must be improved by exercises, which at first cannot be too simple, and must never be continued to the point of fatigue. The feeble power of attention is one of the earliest obstacles to be encountered.

In undertaking the training of the senses we must pay chief attention to touch and sight. By allowing a child to handle suitable objects, we shall cultivate an appreciation of the elementary sensations of hardness and softness, of roughness and smoothness, of heat and cold. Later these lessons may be improved by invoking the aid of the sense known to physiologists as the "muscle-sense." With this help added to that of touch, estimations of size, shape, and weight can be attempted. The child must also be taught the simple properties of objects by the use of its sight. Surfaces which it has recognised by its touch as being smooth or rough should be brought to the judgment of the eyes. For this purpose we shall find it useful to employ articles made of wool, cotton, velvet, or silk. The same holds good of questions of size and shape, and the child should be encouraged to arrange in order a number of balls or sticks of unlike measurements. On other occasions we may throw together on a table objects made in different geometrical shapes. The child is then given one of a selected shape, as, for example, a cube, and is asked to pick out all of similar form that lie before him. The colour-sense must not be neglected, and can be best trained by such simple exercises as matching ribbons or cardboard shapes, sorting coloured beads, and the like. Hearing is less important than either touch or sight, but its training must nevertheless be attempted. Beginning by instruction in elementary sounds such as those resulting from clanging sonorous bodies together-normal babies delight in striking their feeding-spoons against a table or a mugwe should proceed in time to nursery rhymes and simple tunes. These commonly appeal to the weak-minded-indeed, mentally deficients are often peculiarly susceptible to musical rhythm. More exact improvement in hearing results if 
the child is capable of understanding speech or of actually talking.

The next point is the training of the voluntary muscles. Here an exact investigation of the extent of the muscular deficiency, of its nature, and of its cause is all-important. In very many cases there is no actual paralysis, but only tremulousness or imperfect co-ordination. In others paralysis may be severe, and may be associated with rigidity of one or more limbs, or with athetotic movements. We must be careful to adapt the exercises partly to the nature of the muscular defect, partly to the muscles or movements specially involved. Coordination must be improved by such simple kindergarten exercises as threading beads, picture-pricking, or paperweaving. Other useful muscular exercises are dressing and undressing, tying knots and bows, folding paper, arranging articles in order, and, at a later stage, macrami-work. Graduated instruction in drawing simple geometrical forms-straight lines, curves, triangles, squares, and circles-will open the way to shaping letters. Not many weak-minded children will be carried all this way, but the limitation is of small importance. Much more useful than these accomplishments is proficiency in some industrial or manual occupation. The kinds of work most suited to boys are gardening and agricultural employment, carpentry, slojd, and wood-carving. For girls there are sewing, cooking, laundry, and other forms of housewifery. Both sexes can undertake clay-modelling, basket-weaving, or brushwork. It need hardly be mentioned that fewer mentally defectives will be capable of manual work than of industrial work.

The faculty of speech, being dependent on both sensory and motor impulses, demands a certain minimum of intelligence for its development. Not only must words be heard and understood, but the elaborate motor apparatus necessary for articulation must be efficient and under control. There are many idiots who can neither talk nor understand what is said to them. Our efforts to teach these to speak will be wasted. Others can understand what is said to them, and can even acquire a limited vocabulary. The scale ascends to those who can be taught to speak and to converse. Instruction in talking 
must begin with elementary sounds-the labial consonants are usually learnt with least difficulty-and we must be particularly careful to overcome any deficiencies in the muscles of phonation, as, for example, in the lips or tongue. Special gymnastic exercises, devised to bring the defective muscles into action, must be arranged. Names of those objects which are familiar to the child should be the first words to be taught, and the general line of instruction should follow the steps in a normal baby's speech. It is useless to try to hurry children in this matter. They must be allowed ample opportunity to practise every few words that they learn.

In this, as in all aspects of the training of idiots, full advantage should be taken of their innate love of mimicry.

The last form of educational treatment is Intellectual Training. Only the feeble-minded and the higher grades of imbeciles have any capacity for mental work. The arts of reading and writing should never be forced on those who will not be able to profit by them. A weak-minded child who will eventually be capable of earning his living ought, if possible, to be able to write a letter; but another, who will never be allowed to go out into the world, may profitably finish his education when he has learnt to weed the garden.

\section{MORAL INSANITY.}

We have already mentioned the not uncommon association of moral deficiency with mental deficiency, and we have seen that weak-minded children may be characterised by cruelty, cunning, and outbursts of passion. Apart from these subjects, there are children of quick understanding whose moral characters unfit them for social life. At an early period they show a facile inclination to offend against the canons of morality, and, what is worse, they are found to possess natures that are wholly unresponsive to instruction. Precept and example, punishment and reward, fail to convince them of the wrongfulness of their acts. They have neither a sense of shame nor a desire to improve. In almost all normal children, especially in boys, we find a certain aptness to deceive by speech or by act, to appropriate without right of possession, and to commit many 
other offences which, at this age, are venial. But these weaknesses should yield to moral training. In the few children in whom these bad habits remain, we have the class of the "morally insane." At least one example of such children is probably within the experience of every reader. Their natures may be consistently dominated by cunning, by cruelty, or by some other vice. In other examples, a depraved temperament is interjected, as it were, between healthy psychical states. In their lucid intervals these children suggest nothing of the devilishness of their other occasional selves.

Heredity has been held accountable for moral insanity, and without doubt, many of the unfortunates come of neurotic stock. Yet sometimes we see cases of normal, obedient, and docile children becoming fundamentally transformed in temperament after an attack of fever or during the progress of some nervous disease. Epilepsy and injuries to the head are well known to divert the channels of moral action. An interesting series of cases has been collected ${ }^{1}$ in which diphtheria, tuberculous meningitis, and other affections have produced these changes. Thus a good-tempered and obedient child developed a cerebral tumour and became passionate and emotional. Sometimes the mental change after an illness is only temporary, as with a girl whose nature was altered by an attack of typhoid fever, but who eventually returned to her original self.

Morally insane children are a source of profound anxiety and distress to their parents, but their condition is not hopeless, and in some of them the sense of right and wrong can be sharpened by judicious treatment. Once the nature of the condition has been recognised-and the earlier this is appreciated the better-punishment for wrong-doing must be laid aside. It is powerless to develop the moral sense, and often stirs up mud in waters already turbid. But though punishment is inadvisable, we should not protect the child from any unpleasant consequences of his act. On the other hand, rewards for right-doing are of service, and should be promptly made. Perhaps the most satisfactory means of effecting a gradual improvement in these children is to place them, when-

1 Dr G. F. Still, Lancet, 1902, vol. i., p. 1008. 
ever their circumstances allow, under the immediate and constant control of trustworthy governesses or tutors, as the case may be, who realise the nature of their responsibility and the necessity of impressing their characters on their charges. Social intercourse with other children must not be too free, and companions must be selected with judgment. In the later years of childhood, the discipline of school may have a good effect, but the result may be bad, and must be watched very carefully. 


\section{CHAPTER X}

\section{INFANT MORTALITY}

The Age-Factor in Infant Mortality: The First Five Years; The First Year; Infant Mortality, 1851-1907; General Death-rate; Birthrate; Infant Mortality in each Quarter of the First Year; The First Quarter; The First Month; The First Week; The First Day. THE INFLUENCE OF TOWN LifE: Urban and Rural Counties; Density of Population, etc. The Influence of Illegitimacy. Diseases RESPONSIBLE FOR INFANT MORTALITY : Wasting Diseases ; Diarrhœa ; Acute Pulmonary Diseases ; Convulsions ; Tubercle ; Syphilis ; Rickets ; Infectious Fevers ; Death Certification; Statistics of Infant Mortality in the Evelina Hospital for Sick Children, I885-I906; Conclusions.

\section{The Age-Factor in Infant Mortality}

WHEN the physiology of childhood was under discussion, it was pointed out that the changes in an infant's surroundings at birth placed a heavy tax on its adaptive powers. This fact alone suggests that a proportion of the new-born, unable to meet this demand, will not survive their birth for many days. Remembering, further, the dangers to the child during the progress of delivery and the not uncommon existence of deformities, which, compatible with vigorous life in the womb, yet make a separate existence impossible, we shall be prepared to find a somewhat higher mortality in the earliest weeks than later.

On the other hand, the pathogenic influences of the first few years of life do not come into operation at the beginning. They spare the new-born infant, but take an increasing toll at subsequent periods while children are still unable to protect themselves from harm. This risk will be greatest in the year when they are most helpless, but is likely to decrease when they become capable of intelligently making known their wants. The annual mortality would therefore be expected to be heavy at first, and to lighten rapidly in subsequent years. From the 
last annual report of the Registrar-General, we find that in 1907 the total deaths in each of the first five years were as follow:-

Total Deaths in each of the First Five Years.

\begin{tabular}{|c|c|c|c|c|}
\hline 0 to 1. & 1 to 2. & 2 to 3. & 3 to 4. & 4 to 5. \\
\hline 107,978 & 30,036 & II,988 & 7,656 & 5,595 \\
\hline
\end{tabular}

The relative importance of each of these years is better shown by the next table, which gives the numbers of deaths, during each of the first five years of life, per Iooo children born.

Deaths during each of the First Five Years of Life, per 1000 Children born. England and Wales, 1905.

\begin{tabular}{|c|c|c|c|c|c|}
\hline 0 to 1. & 1 to 2. & 2 to 3. & 3 to 4. & 4 to 5. & $\begin{array}{c}\text { Total, } \\
0 \text { to } 5 .\end{array}$ \\
\hline 156 & 40 & I5 & 9 & 6 & 226 \\
\hline
\end{tabular}

More than 20 per cent. of children do not attain their fifth birthday, and, of the deaths, 70 per cent. are crowded into the first year. We must therefore focus our attention on this earliest period.

Though heavy, the above-mentioned infant ${ }^{1}$ mortality of I 56 per thousand births is by no means exceptional. The figures for the last fifty years are practically as high :-

\begin{tabular}{|c|c|}
\hline Years. & $\begin{array}{l}\text { Infant Mortality per } \\
1000 \text { Births. } \\
\text { England and Wales. }\end{array}$ \\
\hline 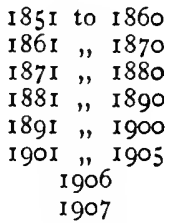 & $\begin{array}{l}\text { I } 54 \\
\text { I } 54 \\
\text { I } 49 \\
\text { I } 42 \\
\text { I } 54 \\
\text { I } 38 \\
\text { I } 33 \\
\text { I } 18\end{array}$ \\
\hline
\end{tabular}

"It is customary to restrict the term "infant" to children under one year of age. 
The loss of life expressed by these rates will be better realised by looking at the absolute numbers of deaths. In 1907, no fewer than I08,000 babies died in England and Wales before their first birthday. In I906, the corresponding figure was I 24,000, and in $1905,120,000$. In the seven years 1900 to 1906 , the total number of infant deaths was close on I,000,000. A harvest of death reaching these proportions is serious in itself, but becomes additionally grave when we learn that while, during the last forty years, the infant mortality has shown only an insignificant fall, the general death-rate for all ages has declined 30 per cent. Thus,

In 1870 the general death-rate was 21.6 per 1000 of the population.

\begin{tabular}{|c|c|c|c|c|}
\hline & & & & \\
\hline I880 & $"$ & $"$ & 19.5 & $"$ \\
\hline I 890 & $"$ & $"$ & $19 \cdot 3$ & $"$ \\
\hline I900 & $"$ & $"$ & $18 \cdot 2$ & " \\
\hline I905 & $"$ & $"$ & $15 \cdot 2$ & $"$ \\
\hline I 907 & $"$ & " & 15.0 & " \\
\hline
\end{tabular}

In conjunction with these returns we must consider the steady decline in the birth-rate, which

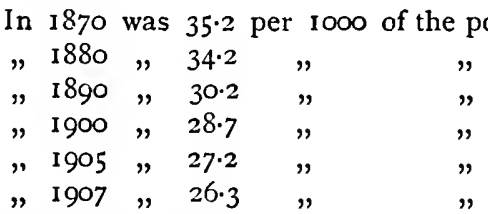

Fewer children are being born every year, and therefore the life of each is increasingly valuable.

The infant mortality given above refers to deaths at any time from birth to the end of the first year, but does not indicate what part of the year is the most fatal. This relative importance is set out in the following table, in which the year is divided into three intervals:-

Infant Mortality in England and Wales.

\begin{tabular}{|c|c|c|c|c|}
\hline & $\begin{array}{c}0 \text { to } 3 \\
\text { Months. }\end{array}$ & $\begin{array}{c}3 \text { to } 6 \\
\text { Months. }\end{array}$ & $\begin{array}{c}6 \text { to I2 } \\
\text { Months. }\end{array}$ & Total. \\
\hline I901 to I905 & 69.63 & 27.77 & 40.42 & 137.82 \\
I906 & 67.62 & 26.95 & 37.93 & 132.50 \\
1907 & 63.97 & 21.30 & 32.35 & II 7.62 \\
\hline
\end{tabular}


Or, if we look at the absolute numbers of deaths instead of the rates, we have the following :-

Total Infant Deaths in England and Wales.

\begin{tabular}{|c|c|c|c|c|}
\hline & $\begin{array}{c}0 \text { to } 3 \\
\text { Months. }\end{array}$ & $\begin{array}{c}3 \text { to } 6 \\
\text { Months. }\end{array}$ & $\begin{array}{c}6 \text { to } 12 \\
\text { Months. }\end{array}$ & Total. \\
\hline 1904 & 67,000 & 28,500 & 41,900 & I 37,400 \\
1905 & 61,855 & 23,008 & 34,228 & I I 9,091 \\
1906 & 63,237 & 25,195 & 35,463 & 123,895 \\
1907 & 58,723 & 19,562 & 29,693 & 107,978 \\
\hline
\end{tabular}

These figures show that the first three months are the most fatal, the deaths within this period equalling those in the remaining nine months. Fifty per cent. of the total infant deaths occur in the first trimester, 20 per cent. in the second, and 30 per cent. in the third and fourth.

Next we must learn at what stage in the first quarter most deaths occur. Until recently this information has not been available, but in the last three reports of the Registrar-General the requisite tables appear for the first time, and from them the following figures are taken:-

Infant Mortality under three months (both sexes). England and Wales, 1905, 1906, and 1907. Proportion of Deaths per I 000 Births.

\begin{tabular}{|c|c|c|c|c|c|c|c|c|}
\hline & \multicolumn{4}{|c|}{ Weeks. } & \multicolumn{4}{|c|}{ Months. } \\
\hline & $\begin{array}{l}\text { Under } \\
\text { I Week. }\end{array}$ & 1 to 2. & 2 to 3. & 3 to 4. & $\begin{array}{l}\text { Under } \\
1 \text { Month. }\end{array}$ & 1 to 2. & 2 to 3. & Total. \\
\hline $\begin{array}{l}1905 \\
1906 \\
1907\end{array}$ & $\begin{array}{l}25 \cdot 16 \\
24 \cdot 99 \\
24 \cdot 39\end{array}$ & $\begin{array}{l}6.08 \\
6.09 \\
5.98\end{array}$ & $\begin{array}{l}5.93 \\
6.23 \\
5.86\end{array}$ & $\begin{array}{l}4 \cdot 58 \\
4.59 \\
4 \cdot 45\end{array}$ & $\begin{array}{l}4 I \cdot 75 \\
4 I \cdot 90 \\
40 \cdot 68\end{array}$ & $\begin{array}{l}\text { I } 3.91 \\
\text { I } 4.13 \\
\text { I } 3.38\end{array}$ & $\begin{array}{r}10.90 \\
11.42 \\
9.91\end{array}$ & $\begin{array}{l}66 \cdot 56 \\
67 \cdot 62 \\
63 \cdot 97\end{array}$ \\
\hline
\end{tabular}

With more than four times the mortality of any other similar period, the first week is by far the most fatal, and accounts for one-fifth of all the deaths in the first year. Further, 
we must add that, in 1906 and 1907 , of the mortality of 24.99 and 24.39 respectively, under one week, I I.77 and II.34 respectively occurred in the first day.

To sum up the age-factor in infant mortality, we may state that, of the whole mortality in the first year,

50 per cent. occurs within the first trimester.

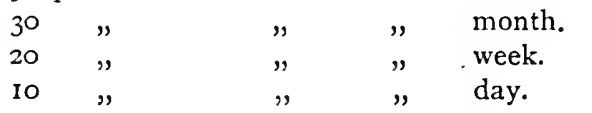

One of the most serious aspects of the matter centres round the new-born babe, which seems so little able to surmount the difficulties that obstruct its path. Some observers have regarded the feebleness of its vitality as a sign of physical deterioration of the race. We shall defer the consideration of this question to the next chapter, and for the present will merely point out that though the fault might lie in this direction, there is also the possibility that the early environment into which many children are born may be responsible for some of this loss of life.

\section{The INFLUENCE OF TOWN LIFE}

We must now turn to another aspect of the matter. Everybody will admit that ill health of every degree is more often seen among the children in towns than among those in the country. Statistical comparisons between the two show what a relatively poor chance of life belongs to the town-bred baby. The infant mortality for both sexes and from all causes was, in 1906 and 1907 respectively,

$\mathbf{1 4 3 . 3 6}$ and $\mathbf{1 2 7 . 6 6}$ for urban counties.

I08.80 and 98.58 for rural counties.

The whole truth is not disclosed by these numbers, since many rural counties contain large towns, and some urban counties include rural areas. A better comparison is made by selecting typical representative counties of each class. Let us choose Dorsetshire,Wiltshire, and Herefordshire-all agricultural 
counties with little or no mining or manufacturing industriesas representative of the one group; and Durham, Lancashire, and Staffordshire as typical urban counties. In the rural group the average infant mortality for the years 190 I to I903 was 91.47 , but in the urban group it was I4S.I7-an excess of more than 60 per cent. If the groups are compared by trimesters, the preponderance is, on each occasion, with the urban counties:-

Average Infant Mortality in Selected Rural and Urban Counties, I90I-1903.

\begin{tabular}{|c|c|c|c|c|}
\hline & & Rural. & Urban. & $\begin{array}{l}\text { Excess per cent. of } \\
\text { Urban over Rural. }\end{array}$ \\
\hline $\begin{array}{l}0 \\
3 \\
6\end{array}$ & $\begin{array}{l}\text { to } 3 \text { months } \\
" \quad 6 \quad " \\
" 12 \quad " \\
" 12 \quad "\end{array}$ & $\begin{array}{l}53 \cdot 56 \\
14 \cdot 49 \\
23 \cdot 42\end{array}$ & $\begin{array}{l}77 \cdot 00 \\
33 \cdot 21 \\
47 \cdot 96\end{array}$ & $\begin{array}{r}44 \\
129 \\
105\end{array}$ \\
\hline
\end{tabular}

By making the comparison, not between rural and urban counties, but between rural counties and towns, we shall find a still more marked contrast.

Deaths per 1000 Births in three Rural Counties and three Selected Towns, 1889-1891.

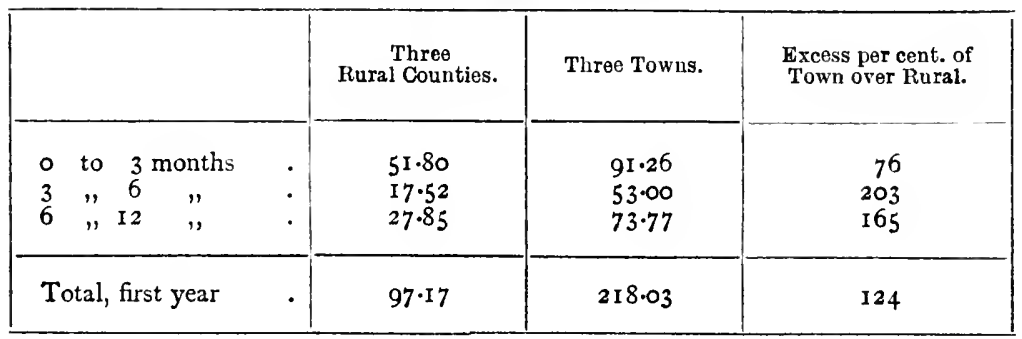

More than twice as many deaths per 1000 infants born occur among the town children as among the country children. The fact that the greatest excess is present in the second trimester suggests that causes other than immaturity must be held responsible for the differences. There are many factors of 
urban life, each or all of which might be blamed for this deadly effect. Overcrowding, insanitation of the poorer quarters of large towns, domestic uncleanliness, poverty, deficient nourishment, ignorance, and neglect are all common features of the town dweller's surroundings. Factory and other work carried on by mothers who should be at home tending their nurslings, plays an important part in swelling the death-rate. Mere density of population has but little bearing on the mortality. Dr Newman has given figures to this effect, ${ }^{1}$ and the returns of the large industrial dwellings point in the same direction. Thus the buildings controlled by the Peabody Donation Fund occupy an area of 33 acres, with a mean density of population equal to 603 persons per acre-or nearly ten times the mean density throughout London; yet the infant death-rate is only 84.9 per 1000 births, compared with 133.0 in the whole of London.

\section{The INFLUENCE OF ILLEGITIMACY}

The other influences of urban life will be discussed later; but we must here observe that though unhygienic surroundings are undoubtedly a prolific cause of infant deaths, there is another factor hardly less serious in its results. This is neglect, whether deliberate or not. In a household already containing more mouths than can be filled, the arrival of a new baby is not always welcomed, and there may be no one to grieve at its premature death. In the poorest quarters of a large city, mothers see children dying round them by the score, one out of every five never reaching a fifth birthday, and this experience must breed a certain callousness to infant life. By no statistical method can we estimate the importance of neglect as a cause of infant mortality, but a sidelight is thrown on the subject by comparisons between the mortality of legitimate and illegitimate children. Whether or not the legitimate child receives a welcome, the birth of an illegitimate infant is not likely to be looked forward to with pleasure. Its survival entails so many penalties on the unhappy mother that only too often she belies her instincts and exposes her child to conditions 
that lead to its death. We shall find eloquent proof of the unfortunate lot of the illegitimate child in the following table :-

Mortality of Illegitimate as compared with Legitinate Infants under one year of age. All causes. England and Wales, 1907.

\begin{tabular}{|c|c|c|c|c|c|c|c|c|c|}
\hline & $\begin{array}{l}\text { Under } \\
1 \text { Week. }\end{array}$ & 1 . & 2. & 3. & $\begin{array}{l}\text { Under } \\
\text { I Mnth. }\end{array}$ & 1. & $2-$ & 3. & \\
\hline Illegitimate . & 40.40 & 10.77 & IO. I I & $9 \cdot 18$ & $70 \cdot 46$ & 28.68 & 23.52 & I9.54 & \\
\hline \multirow[t]{2}{*}{ Legitimate . } & $23 \cdot 73$ & $5 \cdot 78$ & $5 \cdot 69$ & $4 \cdot 26$ & $39 \cdot 46$ & $12 \cdot 76$ & $9 \cdot 35$ & $7 \cdot 7 \mathrm{I}$ & \\
\hline & $\underset{\text { Mnths. }}{\text { Mnthe }}$ & $5-$ & 6. & 7. & 8. & 9. & 10. & I1. & Total. \\
\hline Illegitimate. & I 5.92 & I $2 \cdot 19$ & II $\cdot 38$ & $8 \cdot 4^{8}$ & $8 \cdot 93$ & $7 \cdot 20$ & $6 \cdot 66$ & 6.80 & $219 \cdot 76$ \\
\hline Legitimate & 6.57 & $5 \cdot 94$ & $5 \cdot 90$ & $5 \cdot 53$ & $5 \cdot 39$ & $5 \cdot 23$ & $4 \cdot 87$ & $4 \cdot 72$ & I I 3.43 \\
\hline
\end{tabular}

The next table is taken from one prepared by Dr Tatham, Superintendent of Statistics, Somerset House, of the deathrates of legitimate and illegitimate infants in London and in a group of rural counties :-

Death-rates of Legitimate and Illegitimate Infants per 1000 Births of each. Dr Tatham.

\begin{tabular}{|l|c|c|c|c|}
\hline & \multicolumn{2}{|c|}{ London. } & \multicolumn{2}{|c|}{$\begin{array}{c}\text { Rural Counties } \\
\text { (group of). }\end{array}$} \\
\hline & Male. & Female. & Male. & Female. \\
\hline $\begin{array}{l}\text { Legitimate } \\
\text { Illegitimate }\end{array}$ & $\begin{array}{l}\text { I } 5 \mathrm{I} \cdot 2 \\
\mathbf{2} 89 \cdot 3\end{array}$ & $\begin{array}{l}\text { I I } 8 \cdot 7 \\
264 \cdot \mathrm{I}\end{array}$ & $\begin{array}{l}\text { I25.6 } \\
\text { I90.2 }\end{array}$ & $\begin{array}{r}98 \cdot 8 \\
\text { I66.I }\end{array}$ \\
\hline
\end{tabular}

These figures show that the mortality of illegitimates, both male and female, is, in rural counties, over 50 per cent. higher, and, in London alone, over 100 per cent. higher than that of 
children born in wedlock. Though we must allow that skilled medical or nursing assistance will be more frequently absent at illegitimate births (the mortality for the two classes from injury at birth was $2.2 \mathrm{I}$ and 0.83 respectively in 1907 ), and though we must admit that the mother of an illegitimate child may have to undergo privations from which a husband would protect her, yet these differences alone will not account for the figures. Again, the deaths of an excessive number of illegitimates are ascribed to prematurity (3I.39 illegitimate, $19 \cdot 37$ legitimate, in I907), and to congenital defects (8. I 8 illegitimate, 6.54 legitimate, in 1907), but it is difficult to believe that a purely legal or social bar can affect the physiological or anatomical condition of a child in utero. Convulsions-a meaningless term as a cause of death-is accepted as accounting for a mortality of $2 \mathrm{I} \cdot \mathrm{I} 7$ of illegitimates, as compared with 10.64 of the legitimates (1907). The next table seems to offer the solution of the question. It puts, side by side, the mortalities for both classes of infants from those diseases which are known to be largely the outcome of carelessness and evil surroundings :-

Mortality of Illegitimate as compared with Legitimate Infants under one year of age, 1907. England and Wales (both sexes).

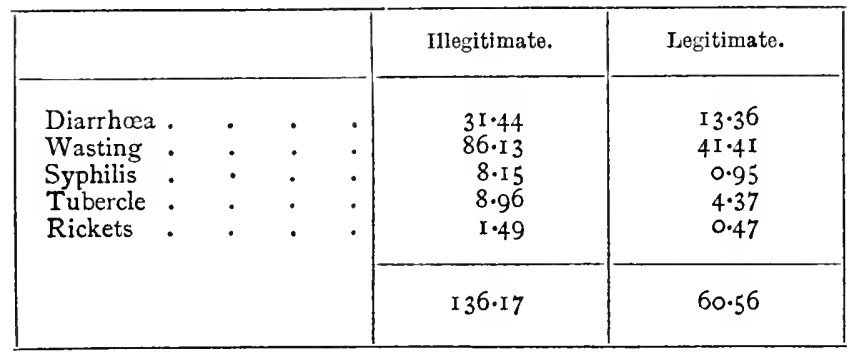

This unequal relation must be ascribed primarily to the exposure of illegitimate children after birth to adverse conditions-in other words, to neglect. This conclusion is supported by the next table, which shows that the differences between the 
two sets of death-rates are less marked at birth than later, when neglect has had time to work its effect.

Table showing the relation between the Death-rates of Illegitimate and Legitimate Infants. The figures represent the Illegitimate Mortality as compared with a Legitimate Mortality of Ioo at each specified age. Calculated from a Table by Dr Tatham.

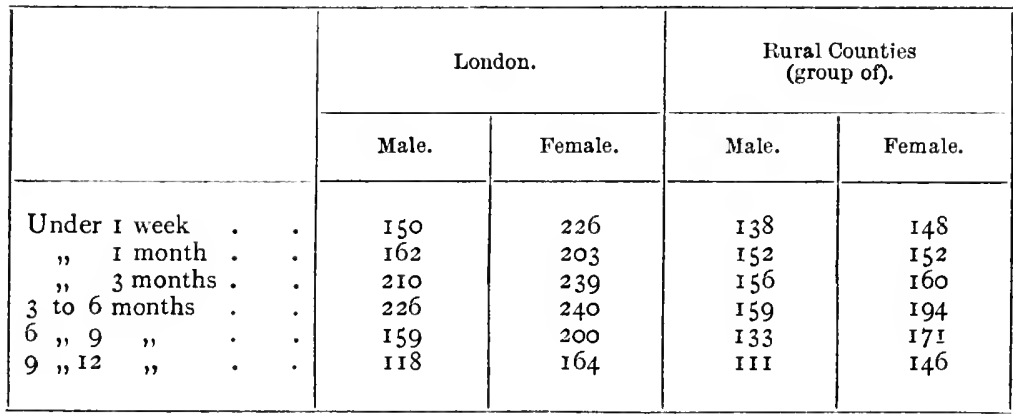

An additional point worthy of note is that our country is not unique in this record. In New South Wales, for example, during the years I895-I902, the mortality of legitimate infants under three months was $49^{\cdot} \mathrm{I}$, but the corresponding figure for illegitimate infants stood at 145.9 . Yet in spite of this excessive difference (a ratio of $I: 3$ ), the mortality of illegitimates in their second year was only $I \frac{1}{3}$ that of legitimates, and in the third year the inequality had entirely disappeared. ${ }^{1}$

\section{The Diseases Responsible for Infant Mortality}

Thus far we have dealt with infant mortality in its more general aspects. The question, however, is essentially a medical one, and we must now ascertain what diseases are responsible for the deaths. These diseases are remarkably small in number, and a general survey of their relative importance may be taken from the following list :-

1 Report of Inter-Departmental Committee on Physical Degeneration, vol. i., p. I 34 . 
Causes of Infant Mortality for England and Wales (both sexes), 190I-1905, I906, and 1907.

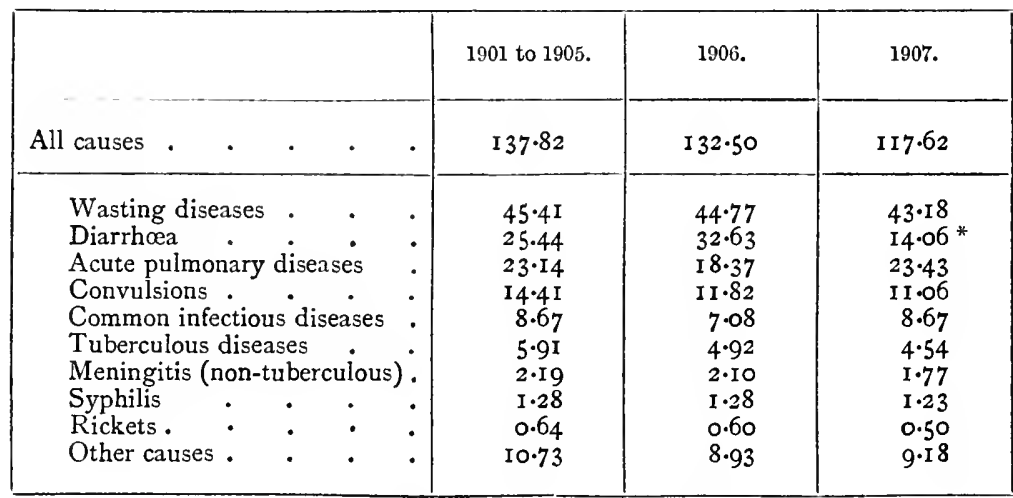

* The mortality in I907 from diarrhœa was exceptionally low, on account of the large rainfall in the summer.

The heading "wasting diseases" includes premature birth, congenital defects, injury at birth, want of breast-milk, starvation, atrophy, debility, and marasmus. Acute pulmonary diseases include pneumonia, broncho-pneumonia, and laryngitis.

The following are the points in the above table that may be specially emphasised. From 70 to 80 per cent. of the whole mortality is included under the four headings of wasting diseases, diarrhœa, acute pulmonary diseases, and convulsions. Tubercle is held responsible for only 4 per cent. of the deaths. Syphilis and rickets-two of the commonest diseases of infancy-take quite a subordinate place in the total mortality.

Next we must make ourselves acquainted with the figures for successive trimesters. The causes of death in the first and in the second quarters and in the last half of the year are arranged in the table on the opposite page. The figures will repay careful study.

The heaviest mortality is ascribed to prematurity. This includes congenital defects and injury at birth, and is responsible for 20 per cent. of the total. The influence of 


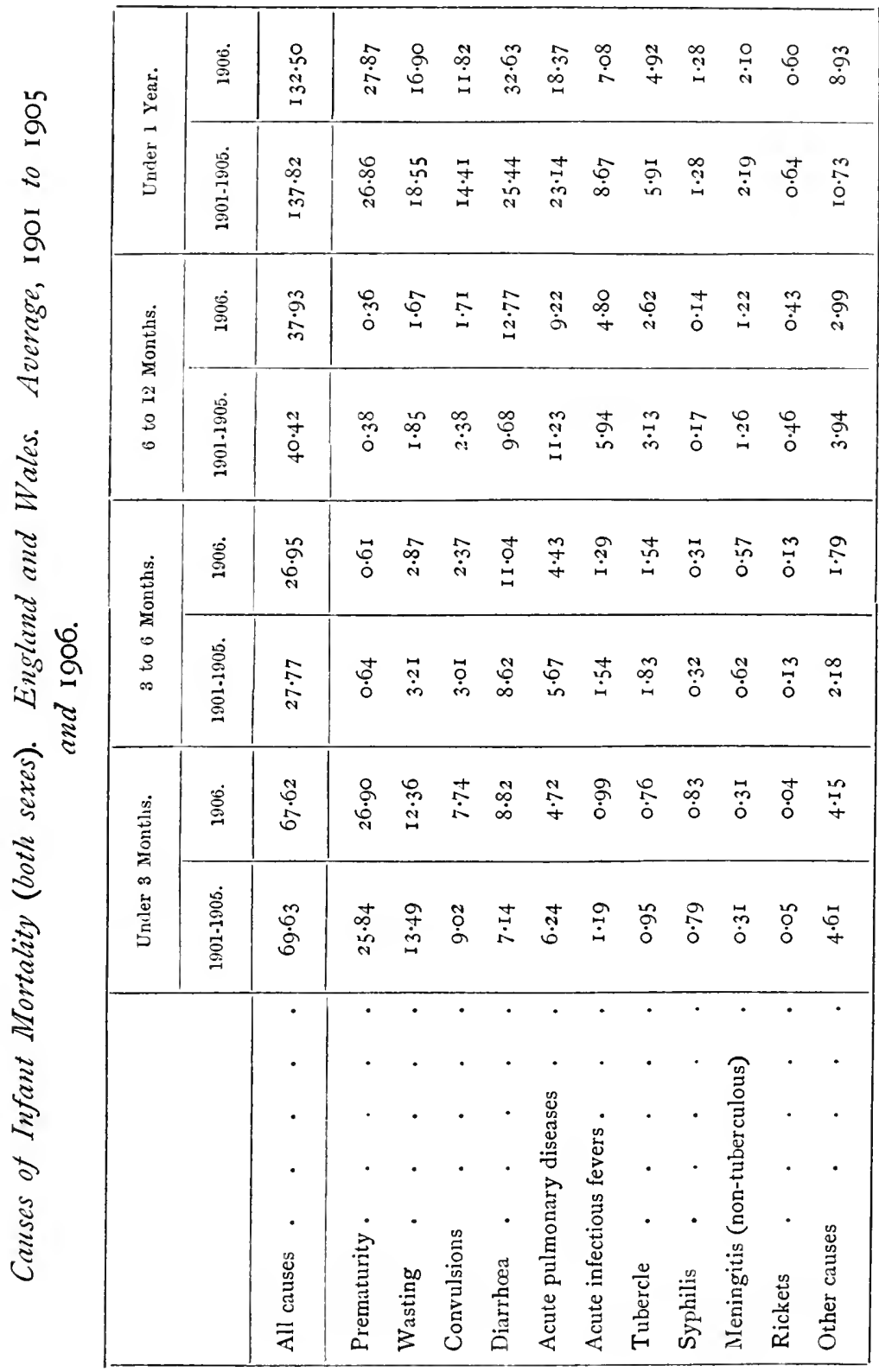


this cause is necessarily restricted to the earliest weeks of life. The condition next in importance is diarrhoa, which, beginning with a mortality of 8.82 in the first trimester, rises to $\mathrm{Ir} .04$ in the second, and to 12.77 in the third and fourth. This large increase accords with our clinical knowledge that diarrhcea is the result of improper feeding, and will therefore be least prevalent in the months when most infants are at the breast. The third most important item is acute pulmonary disease, which, standing at 4.72 in the first period, and at 4.43 in the second, amounts to 9.22 by the end of the year. Laryngitis is comparatively insignificant in producing these figures, which are due to pneumonia and broncho-pneumonia in almost equal proportions. Deaths from convulsions are high in the first trimester (7.74), but quickly sink to 2.37 and $1.7 \mathrm{I}$ in the subsequent periods. We have already commented on the value of the diagnosis "convulsions" as an indication of the cause of death.

With regard to the remaining headings, the figures relating to infectious diseases deserve closer attention. Beginning as low as 0.99 and $\mathrm{I} .29$ in the first and second quarters respectively, they leap up to 4.80 in the last half of the year. The diseases comprised under this heading are: scarlet fever, measles, diphtheria (with croup), small-pox, chicken-pox, and whoopingcough. The mortality from each of these, with the exception of small-pox, increases enormously for infants over six months of age. Thus, contrasting the increase in 1906 from the first trimester to the third and fourth, we find that chicken-pox rises from nil to 0.05 , diphtheria from 0.02 to 0.32 (a sixteen-fold increase), scarlatina from nil to 0.09 , and measles from 0.06 to 2.03 (a thirty-fold increase). In a total mortality from infectious diseases of $7 \cdot 08$, no less than $6.5 \mathrm{I}$ is attributed to whoopingcough and measles ( $4 \cdot 25$ and $2 \cdot 26$ respectively), while diphtheria, scarlet fever, and small-pox together are responsible for only 0.50. These figures recall the fact that neither whooping-cough nor measles is a notifiable disease, and their treatment is not undertaken by our municipal fever hospitals. Infant patients are left to the primitive care of their homes, with the result that, in 1907,7487 deaths from these diseases were registered 
for infants under one year, as compared with 354 deaths from scarlet fever and diphtheria.

Thus far we have followed the official statistics. Their practical value is measured by the accuracy of death-certification. No one familiar with the fatal diseases of infancy will fail to detect a want of harmony between these official returns and his own experience in the ward or sick-room and in the postmortem room. He will be specially struck by the relative insignificance in the mortality tables of certain of the commoner yet more serious diseases of infancy, and he will probably draw the conclusion that figures based on ordinary death-certification give only an imperfect insight into the fatal conditions of the first year.

It is not enough to study the front of a death-certificate. We must look behind it to learn the whole story, and in matters of infant life the statistician must go hand in hand with the clinician and the pathologist. While the value of infant mortality figures depends on exactness of certification, the value of this latter is measured by the precision of diagnosis. Children, especially infants, react to disease in ways that are peculiar to the young, and their symptoms are often anomalous. On this account diagnosis is difficult, and many cases which are clinically obscure will escape recognition. In infantile tuberculosis, for example, we have a disease which, though easy to suspect, is often among the most difficult to identify during life; and many cases must escape diagnosis unless submitted to post-mortem examination.

Further, we must understand that the death-rate ascribed to a disease is often an imperfect index to its real mortality. An affection such as rickets, which takes a relatively unimportant place in the official tables, is, from the clinical aspect, one of the more serious conditions of late infancy. It is not often directly fatal, but is indirectly responsible for numerous deaths. ${ }^{1}$ It

1 One observer has estimated that 30 per cent. of all sick children under two years are the subjects of rickets, and in a series of 492 such cases, 36 per cent. were infants under one year. (Dr Gee, St Bart.'s Hospital Reports, vol. iv., p. 69). 
leads to one of two complications-diarrhœea and bronchopneumonia-which are among the most frequent causes of infant mortality. Yet the deaths in these cases are attributed to the complications, and not to the rickets, the mortality from which remains deceptively low.

Often deaths are certified as due to a prominent symptom, and the disease itself is overlooked. Thus "convulsions" stands high in the tables on pp. 228 and 229 , but the term expresses merely a symptom which is associated with many different diseases. For this reason its use in death-certification is specifically deprecated by the Royal College of Physicians, ${ }^{1}$ yet to this one cause no less than Io per cent. of the total infant mortality is ascribed. If greater accuracy were possible, this large proportion, which represented no less than IO, I5O deaths in I9O7 alone, would be distributed under other headings, such as diarrhœa, pneumonia, fevers, tuberculosis, meningitis, syphilis, or rickets, in each of which convulsions not infrequently occur.

Another symptom which figures prominently as a cause of death is wasting. Although, in the present state of our knowledge, wasting, or marasmus, is regarded by some as a separate clinical entity, it commonly represents merely a symptom of some underlying disease. Though this primary cause may be detected during life, it is often discovered only at the autopsy; and where this examination is not made, deaths would be recorded as due to wasting.

Broncho-pneumonia is another important item in the mortality returns. It may indeed be primary, but veryoften is the terminal complication of some other disease. It frequently follows measles or whooping-cough, and is specially liable to attack the subjects of rickets. When it does follow any of these three, it may not end fatally until some weeks or months after the beginning of the original illness. In this event, death may be recorded as from broncho-pneumonia. ${ }^{2}$ Finally, broncho-pneumonia may

1 Nomenclature of Diseases, 4th edition, 1906, p. 3 I.

${ }^{2}$ In the official returns each death is entered under the heading of the condition of longest standing that is mentioned in the death-certificate. When, however, the primary condition is not mentioned, the death must necessarily be entered under another heading. 
be tuberculous, but is often not recognised as such without an autopsy.

In brief, we see that the official returns must suffer from a certain degree of inexactness. Ten per cent. of the total deaths are attributed to "convulsions," which is a symptom possessing no exact pathological significance. Another I2 per cent. are ascribed to the equally vague cause "wasting," "marasmus," "debility," or "atrophy." Therefore in more than 20 per cent. of the total mortality, the accuracy of the cause of death is open to serious objection. The importance of rickets is undervalued, partly because of the frequency with which rickety infants die in convulsions, partly because the deaths of many fatal cases are ascribed to diarrhœa or bronchopneumonia. Broncho-pneumonia itself complicates diseases such as rickets, measles, whooping-cough, and gastroenteritis-the importance of which is therefore masked. Finally, cases of tuberculous broncho-pneumonia may escape diagnosis, and thus lead to an underestimation of the importance of tubercle.

With a view to obtaining statistics freed as far as possible from the above objections, I have analysed the causes of death in a series of infants who died in the Evelina Hospital for Sick Children. ${ }^{1}$ Though the total number of these deaths is barely one per cent. of the total number in the annual returns for England and Wales, the smaller series possesses the special advantage that infant death-certification is considerably more exact in a hospital where there is every clinical facility for accurate diagnosis, supplemented by the crucial test of postmortem examination.

The figures are based on the records of 1202 consecutive infant deaths from Ist January I885 to 3 Ist December 1906. The majority of these have occurred in the last fifteen years, more than half taking place in the last eight years. The diseases responsible for these deaths, and the number of infants dying from each, are shown in the following table :-

1 The analysis that follows is taken from my paper on "Infant Mortality" in the Proceedings of the Royal Society of Medicine, February 1909. 
Infant Deaths in the Evelina Hospital for Sick Children, I 885 to 1906.

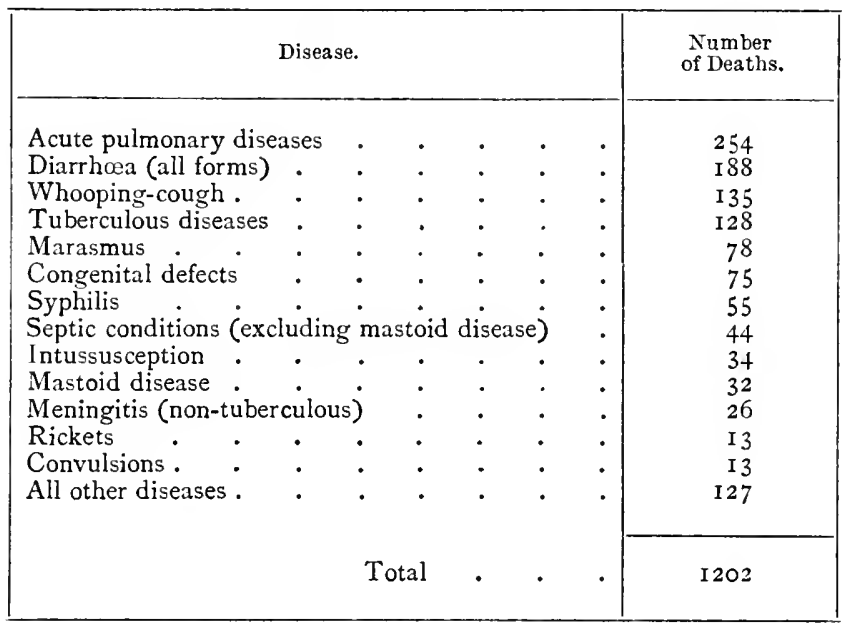

A word is needed in explanation of the method followed in tabulating these cases. In many infantile diseases, more than one pathological condition, each sufficient to account for death, is present, and doubt might arise on this score. For example, many cases of acute gastro-enteritis develop broncho-pneumonia, while in certain cases of broncho-pneumonia severe diarrhœa may be a terminal complication. Each case has been considered on its merits, and from the point of view of preventive measures. The practice followed has been to record complications under the name of the primary disease, but when a later disease has become grafted on an earlier disease, it has been entered under its own heading. Thus deaths from broncho-pneumonia complicating whooping-cough will be found under "whooping-cough," but deaths from tubercle subsequent to whooping-cough, appear under the title "tuberculous diseases."

In the next table, the Evelina figures, expressed in percentages of the total number, are placed, for comparison, in a parallel column to corresponding figures from the Registrar-General's 
report. ${ }^{1} \quad$ The items prematurity, measles, and whooping-cough have been omitted for the following reasons:-Premature infants rarely find their way into a children's hospital, and deaths from prematurity do not often figure in the hospital records. Cases of measles and of whooping-cough are either not admitted into a hospital at all, or only under special conditions. The number of deaths from whooping-cough is inordinately large in the Evelina statistics, because for many years a special ward has been reserved for the treatment of this disease. Excluding these three items in each series, we obtain the following percentages for the remaining diseases:-

Table showing in percentages the Deaths, from specified causes, of Infants in the Evelina Hospital for Sick Children, and in England and Wales, for 1905.*

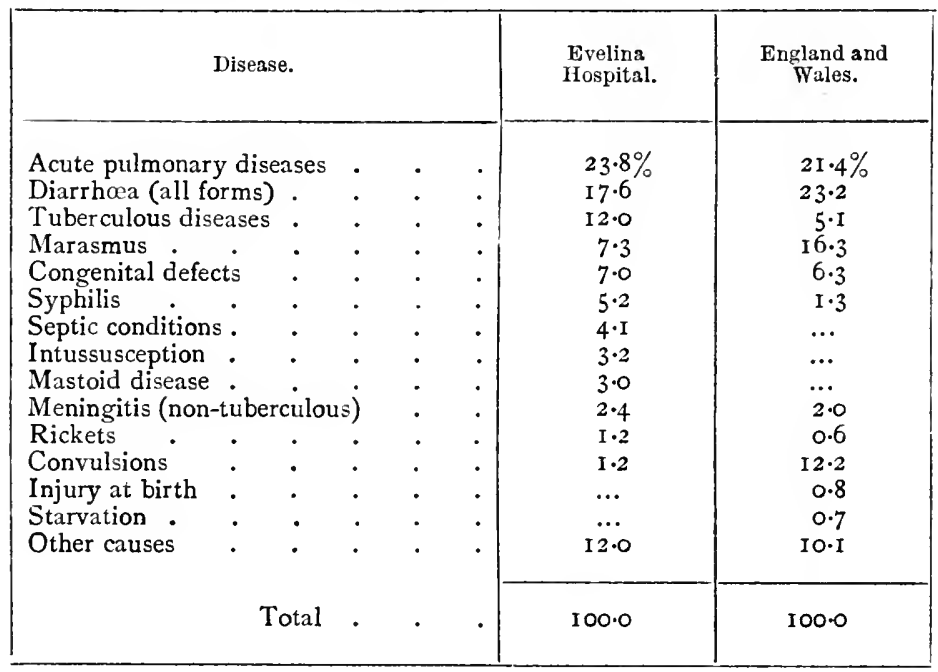

* The returns for 1905 have been selected in preference to those of 1906 or 1907 , because, after excluding the three conditions mentioned above, the total infant mortality under each heading happens to express almost exactly the percentage as given in the table.

1 In a general hospital for adults this comparison could hardly be made, because chronic conditions are, as a rule, not admitted. In a children's hospital this factor has but little value as affecting infant deaths, because 
From the above, we observe that while many close correspondences are found in the two columns, a number of striking differences exist. Congenital deformities, which in the majority of cases are recognised without difficulty, approximate closely. The figures for acute pulmonary diseases show no great differences. With regard to diarrhoea, the difference becomes more marked, and the hospital returns fall 24 per cent. below the official figure. This relation is not difficult to account for. A considerable proportion of the deaths from this disease result from epidemic gastro-enteritis or summer diarrhœa. This disease is rife for a few months only, and during this time the demand on the accommodation in children's hospitals far exceeds the supply. A selective factor therefore arises. Serious cases are not infrequently refused admission, and the hospital mortality under this heading is artificially limited. A second reason is found in the ease with which diarrhœea is recognised, combined with the not uncommon difficulty of ascertaining its exact cause. As a result, cases will be included under the heading of diarrhœa, though with the help of an autopsy their special nature would be seen.

When we come to the items next in importance in the Registrar-General's returns, we find an excessive divergence between them and the Evelina Hospital figures. Marasmus stands at 16.3 and 7.3 in the two columns-a proportion of more than two to one. So large a difference obviously requires some explanation, especially as the mortality of 16.3 represents over I5,000 dead infants. We have already mentioned the unsatisfactory nature of the term marasmus, with its official synonyms atrophy and debility. These terms are employed in connection with diseases in which wasting is merely a prominent symptom. Tuberculous infections, congenital the chronic diseases that are fatal within the first year are very few in number. Such as there are reduce their subjects to so emaciated a state that they become suitable cases for admission. A representative series of all fatal infantile diseases may therefore be looked for in a children's hospital. Further, the accessibility of a hospital such as the Evelina, which lies in the midst of one of the poorest parts of London, enables all cases, no matter how acute, to be brought for treatment. At any given time the inmates will reflect the medical condition of the surrounding neighbourhood. 
syphilis, and improper feeding are some of the conditions which most often reduce infant patients to the more advanced grades of wasting. Many deaths from these causes are undoubtedly registered as due to wasting; whereas only those deaths in which wasting forms the whole picture, etiologically, clinically, and pathologically, should be included under this heading. The nature of the condition is perhaps better expressed by its American title of "primary infantile atrophy."

The general tenor of these criticisms applies even more forcibly to deaths from convulsions. Under this heading we find a mortality in England and Wales of I2.2, representing over II,000 deaths. At the Evelina Hospital we find that only $\mathrm{I} \cdot 2$ per cent. of the deaths are thus diagnosed. The official figure is of no service in advancing our knowledge of the fatal conditions of our infant population, but on the contrary actually misleads us. It hides under a meaningless designation, important and fatal conditions, the returns for which are therefore underestimated.

When the community has set a proper value on the life of an infant, such terms as "convulsions" and "wasting" will no longer be accepted in death-certification. If no more satisfactory explanation of a child's death can be given from the clinical evidence alone, further steps should be taken, as is now done with adult deaths. By this means a far-reaching and practical measure will be provided to diminish the present excessive loss of infant life.

Syphilis and rickets must be next considered. Each occupies a comparatively insignificant place in the official returns. Syphilis is one of the diseases that is most frequently seen clinically and is often fatal, especially in the earliest months of life. Yet in the whole of England and Wales, for 1905, it was held responsible for only I 200 infant deaths-little more than three a day. It is not easy to harmonise these figures with hospital experience. Probably a large number of fatalities from syphilis are attributed to one of two other conditions. In the first place, the large figure that represents the mortality from prematurity $(20.31)$ will include the deaths of many new- 
born children who are the infected offspring of syphilitic parents. In I905, I9,000 babies died from prematurity within three months of their birth, but only Soo are stated to have died from syphilis. In the second place, syphilitic children are usually emaciated, and their deaths are entered as due to marasmus, partly because the primary cause is obscure, partly, perhaps, to spare the parents' feelings.

With regard to rickets, the official and the hospital returns are 0.6 per cent. and $\mathrm{I} \cdot 2$ per cent. respectively. This difference is to be attributed to the readiness with which rickets, as a cause of death, is overshadowed by its terminal complicationsnamely, diarrhœa and broncho-pneumonia. But even the larger figure, I.2 per cent., must not be taken as fully expressing the fatal importance of rickets when acquired in infancy. The end is delayed in many cases until the second year, and the deaths in these cases are necessarily excluded from the infant mortality.

Attention must next be drawn to a purely surgical condition -namely, mastoid disease. Deaths from this cause are not shown separately in the official infant mortality returns, but in I907, they numbered 214 , which represents a mortality of 0.3 . At the Evelina Hospital, on the other hand, they number $3 \cdot 0$ per cent. of the total mortality. The immediate cause of death in many of these cases was septic meningitis. It is interesting to note that the number of deaths from this disease increased rapidly from a minimum in the earliest months. Six per cent. occurred in the first three months; I8 per cent. occurred in the second three months; and 72 per cent. in the last six months. We must therefore regard septic processes in the middle ear as one of the more serious menaces to later infant life. ${ }^{1}$

The last cause of infant mortality that requires our special attention is tubercle. The various forms of tuberculous infection account for a mortality of 5.17 in the returns for England and Wales. At the Evelina Hospital the percentage

1 In 94 consecutive autopsies on children of all ages, but exclusive of those with septic meningitis, Dr Still found pus in one or both middle ears no less than forty-nine times ( 52 per cent.). 
is more than double this-namely, I2.0. Here, again, some explanation is required, especially as the hospital figure includes only those cases in which tubercle was the actual cause of death. It excludes those in which, though a tuberculous lesion was found post-mortem, death resulted from some other disease. We shall find the simplest explanation in the tendency of tuberculous disease to simulate non-tuberculous diseases of infancy. For example, tuberculous disease of the lungs is, in infancy, rarely an excavating process restricted to the apices as in adults. It is usually an irregularly scattered affection clinically suggestive of an "acute pulmonary disease." We can the more readily understand this confusion by remembering that tubercle of the lung may itself be associated with a simple broncho-pneumonia. Similarly, abdominal tubercle may succeed in passing itself off as one of the forms of "diarrhœa." Again, cases of general tuberculosis and of tuberculous meningitis may find permanent record as examples of "convulsions." On the other hand, it may be urged with truth that simple broncho-pneumonia and simple diarrhœa often have their bona fides suspected of a tuberculous taint. But these cases have a tendency to recover, and therefore have less effect on questions of mortality.

We must probably admit that 12 per cent. more nearly represents the real mortality from tubercle than does $5 \cdot 17$ per cent., but even this higher figure-as indeed all computations of the infant mortality from tubercle-underestimates the frequency with which life is destroyed by tuberculous infections incurred during infancy. This point is made clear by two facts. First, tubercle is commoner in the latter half of the first year than in the former half. Second, the disease is not as a rule fatal until some weeks or months after the initial infection. From these premises we can conclude that some cases dating from the third quarter of infancy, and many cases dating from the fourth quarter, will survive beyond the age of one year. Their subsequent deaths will not affect the infant mortality. The full importance of tubercle as a factor in infant mortality is more clearly revealed by statistics dealing with deaths up to 
fifteen months, or even up to eighteen months. The number of such deaths at the Evelina Hospital was 177, of which 49 occurred between twelve and eighteen months. Seeing that infection precedes death by some considerable period, we must admit that many of these 49 infants were infected during their first year. 


\section{CHAPTER XI}

THE CONDITIONS OF INFANT LIFE

CONDITIONS AT BIRTH: Deaths in the First Month; Injury at Birth; Congenital Defects. IMMATURITY AS A CAUSE OF INFANT MORTALITY : Physical Deterioration; Industrial Employment of Women; Want of Food; Heredity; Notification of Births; Postmortem Examination of Stillborn Infants ; Legitimacy and Illegitimacy ; Parental Neglect; Preventable Deaths. Conditions OF LATER INFANT LIFE: Infant Health, Good Food and Cleanliness; Maternal Neglect; Infant Life Assurance; Baby-farming; Mothers who "go out"; Dirt ; Improper Feeding; Infantile Diarrhœea; Breast versus Bottle; Tuberculous Disease. The Remedies: Legal Remedies; Social Remedies; Infant Mortality merely one aspect of the problem of the Poor; Education of Mothers; Lessons in Baby-rearing; Health Societies; Voluntary and Municipal Effort; Pure Milk Supplies; Prohibition of Industrial Labour for Women after Confinement ; Day Nurseries.

THE great interest that has been aroused in the last few years in matters concerning childhood is being rightly focussed on infancy as the period when most harm is wrought. We shall therefore be justified in considering in some fullness the circumstances of infant life from the standpoint of health and disease.

\section{CONDITIONS AT BIRTH}

The figure that stands out in highest relief from a picture that has many unhappy details is that of Death awaiting the new-born child. As we saw in the last chapter, the mortality within the first month of life in England and Wales in 1905, 1906, and 1907 (the only years for which the numbers are available) was $4 \mathrm{I} \cdot 75,4 \mathrm{I} \cdot 90$, and 40.68 respectively per 1000 births -about one-third the total mortality of the first year. Of this 
number, thirty-one represent deaths from causes which are comprised under the official heading of "wasting diseases"namely, premature birth, congenital defects, injury at birth, debility, atrophy, and marasmus. To these half-dozen causes are to be attributed three-quarters of the death-rate among the new-born. This fact can at first sight be regarded only with grave disquietude, and it compels us to seek an explanation of the prominence which these conditions occupy in the death-roll of early infancy.

The reasons commonly advanced are two in number. First, it has been held that the race has physically degenerated, and as a result, is producing an excessive proportion of feeble children who are without the vitality to surmount the natural obstacles incidental to a separate existence; second, that the health of women during pregnancy, especially in its later months, is being deeply undermined by the conditions of modern life, whereby their offspring are prevented from attaining their full development. No conclusion can be more serious than one which impugns the fertility of the race, and if once the truth of the accusation were established, no social problem should take precedence of this, which exposes decay in the very foundations of the State. The question is one of such importance that it is necessary to scrutinise the figures representing the neonatal mortality, in order that we may discover whether they will bear this interpretation, or whether factors other than the two above mentioned may, in part at least, be concerned in setting a narrow limit to so many lives. At the outset we can clear the ground by considering the two conditions, injury at birth and congenital defects, as these possess no very intimate connection with the other four conditions included under the head of wasting diseases.

(a) Injury at Birth.-The mortality from this cause in each of the last three years was $0.76,0.93$, and 0.89 respectively, and occurred almost wholly in the first month of life. Such injuries are due directly or indirectly to pressure of the maternal tissues on the child. They are brought about by a disparity of size between the child and the vice parturientes, and indicate either that the one is too large or the other too small. The former 
relation, provided it arises, as is usually the case, from physiological and not from pathological causes, cannot be regarded from the eugenic standpoint with apprehension. The latter condition-a child of normal size, but straitened maternal channels-is a common one, and provides the most frequent cause of birth-injury. It depends not on any question of the fertility of the race or the vitality of the child, but on the size and shape of the pelvic bones. These, however, have assumed their permanent form long before pregnancy occurs. Injury at birth, therefore, cannot be ascribed to the impaired health of the mothers during pregnancy, nor, since the development of the children in these cases is at any rate not below the average, to the physical degeneration of the race.

(b) Congenital Defects.-These were responsible, within the first month, for a mortality of $4.7 \mathrm{I}, 4.82$, and $4.9 \mathrm{I}$ per 1000 births in 1905,1906 , and 1907 respectively. In attempting to estimate the possible influence of the physical deterioration of the parents on the production of congenital defects, we must recognise that a proportion of the latter are undoubtedly due to the immediate intra-uterine surroundings of the child, and further, that "there are probably many congenital defects which, if they could be traced back to their origin, would be found due to mechanical interference." 1 Again, while a connection can be readily understood between physiological deterioration in the parent and feeble growth or vitality in the child as a whole, it has yet to be shown that the former can be held responsible for local deformities in the child (such as imperforate anus, spina bifida, meningocele, ectopia vesicæ, hydrocephalus, deformed heart, atelectasis pulmonum). Yet it is these local deformities that account for the large percentage of deaths from congenital defects. Finally, if degeneration in the parents produced deformity in their young, it should be found that where one child was born deformed, others of the family should be similarly afflicted: yet this is not the case.

No more obvious is the influence of maternal conditions of life during the later months of pregnancy. Malformation, in the majority of cases, takes its origin at comparatively early

1 Manual of Pathology, Coats, 1903. 
stages in the development of the child, ${ }^{1}$ and therefore we cannot look to the conditions of the mother in the later months of pregnancy as responsible for their production. The majority of the varieties of fatal congenital deformities, and the large majority of the congenitally deformed, are the outcome of conditions special to the fœtus, on which the maternal health in the later months can have practically no influence.

If we exclude the figures for injury at birth and for congenital defects, we find remaining a mortality within the first month of birth of 25.74 in 1906 , which may be attributable to physical degeneration or to maternal conditions in late pregnancy. This number is made up of a mortality of 18.38 due to premature birth and of $7 \cdot 36$ due to atrophy, debility, or marasmus. Though these figures err in all probability on the side of slight excess, inasmuch as various deep-lying congenital defects or injuries may have been overlooked in some cases, they may be accepted as approximately representing the maximum mortality in the first month from causes which can be referred to either of the two above-mentioned conditions. The next step will be to enquire how far the deaths from premature birth, atrophy, debility, and marasmus (which for convenience may be grouped under the term "immaturity") are the result of these factors, and how far they are influenced by forces extrinsic to the child and arising at or shortly after birth.

Immaturity as a cause of Infant Mortality in the First Month.-So much prominence has been given to the supposed decadence of the present generation, that some, perhaps, are inclined to accept its existence as proved. The question is necessarily a most intricate one. The Interdepartmental Committee on Physical Degeneration ${ }^{2}$ found, however, that the facts and opinions they collected did not support the belief in any general progressive physical degeneration. In face of this conclusion, we must look elsewhere to account for the 25.74 mortality.

Those who have placed the blame on the maternal surroundings during the last month or two of pregnancy have 
directed their special attention to the industrial employment of married women. It has been pointed out that during this time the weight of the fotal child doubles itself-increasing from $3 \frac{1}{2}$ to $7 \mathrm{lb}$. - and that, to meet the demand of so rapid a rate of growth, hygienic surroundings for the mother are more essential at this period than at any other. This reasoning, however, is not convincing, for the following figures ${ }^{1}$ show that the relative rate of growth of the child in utero is actually slowest in the last two months of pregnancy as compared with the earlier months.

From the beginning to the end of pregnancy, the weight

\begin{tabular}{|c|c|c|c|c|c|c|c|c|c|c|c|}
\hline At end of & I & month & . & . & - & Weight & tus is & . & . & 0.09 & $\mathrm{Oz}$ \\
\hline, & 2 & ," & . & - & . & , & ", & - & , & 0.55 & " \\
\hline$"$ & 3 & $"$ & • & . & . & $"$ & $"$ & • & . & 3.00 & $"$ \\
\hline ", & 4 & $"$ & . & . & - & $"$ & , & • & . & $7 \cdot 50$ & $"$ \\
\hline ", & 5 & $"$ & . & . & - & $"$ & $"$ & . & - & 16.00 & $"$ \\
\hline$"$ & 6 & $"$ & . & . & . & " & $"$ & . & . & 36.00 & ", \\
\hline$"$ & 7 & $"$ & . & . & . & $"$ & $"$ & . & . & 52.00 & , \\
\hline$"$ & 8 & $"$ & . & . & . & ", & $"$ & . & . & 76.00 & $"$ \\
\hline " & 9 & $"$ & • & & . & $"$ & " & • & . & I I 2.00 & $"$ \\
\hline
\end{tabular}

has been variously estimated to increase from 2500 times $^{2}$ upwards. Growth is fastest in the first three months-more than a thirty-fold increase-and more changes are brought about in the embryo in the first three months than in the remaining six months. It would seem imprudent, therefore, to concentrate our special attention on the last months of pregnancy. Important as this time undoubtedly must be in many respects, it can hardly exert as much influence as the earlier months, for at the later period the child's development is already almost complete. We can hardly doubt that it is necessary to look to the health of a mother throughout the whole period of gestation, and that it is not by special attention during the last few weeks that we can make up for errors or

1 Manual of Midwifery, A. L. Galabin, M.D., I904.

2 The Child, A. F. Chamberlain, 1900, p. 72. Professor Stanley Hall states that the increase in weight during the first three months is $400,000,000$ per cent., in the second three months 5182 per cent., and in the last three months 252 per cent. (Adolescence, 1904, vol. i., p. 3). 
neglect in the earlier and more important stages. Factory work or shortage of food has, it may be admitted, no good effect on a mother during the time immediately preceding the birth of her child, but we have not yet obtained data to justify us in attributing the early mortality of infancy to these special conditions. On the contrary, all who have come in contact with the new-born children of the poorest must have been repeatedly struck by the fine development and vigour of babies whose mothers have worked hard up to the time of their confinements, and are perhaps themselves pinched and thin from obvious lack of food. Often it seems as if the infant had flourished at the expense of its parent.

The report of the Registrar-General for I905 affords explicit support to these remarks. "There appears," it states, " to be a general belief that the employment of married women in industrial occupations is a cause of increased infant mortality. The extent to which such employment is prejudicial to infant life is, however, difficult to determine." A table is given to contrast the birth-rates and infant mortality in fifteen towns (Group A) where only 4 to 8 per cent. of the married or widowed women were engaged in occupations, and those in fifteen towns (Group B) where from 20 to 36 per cent. of the married and widowed women were similarly occupied. "The figures," the report continues, "afford presumptive evidence that the industrial employment of married women has had with few exceptions an adverse effect on the birth-rate... The connection between infant mortality and the employment of married women does not, however, appear to be so intimate. ... There are several towns in Group B which ... do not have an unduly high rate of infant mortality; while on the other hand, there are three mining towns in Group $A$ that are conspicuous examples of the reverse condition."

Neither physical degeneration nor industrial employment in late pregnancy satisfactorily accounts for the mortality of $25 \cdot 74$. Heredity and antenatal physiology throw but little light on the problem, and it is probable that the chief factor in the production of immaturity has yet to be discovered. It may be long before we understand the cause and learn how to circumvent its effects, 
but in the meantime further information may be looked for in another direction. It is probable that when the Notification of Births Act, 1907, has been adopted throughout the country, and the birth of each child is notified within a few hours of its occurrence, so that a watchful eye can follow the infant's progress, a marked effect on the infant mortality returns will be produced. Apart from this legal test, there is another means by which our knowledge on the subject might be greatly improved-namely, by medical investigations along an at present almost completely neglected line. It is a matter for comment that while the exact causes of the death of children and adults are being daily ascertained by post-mortem examination, this procedure is practically never applied to a still-born child or to a baby that dies within a few hours or days of its birth. A systematic examination of all such infants could not fail to provide exact pathological information. It would limit the use of such vague terms as "atrophy," "debility," "marasmus," and would be of greater service in solving the problem of immaturity than any speculations as to possible causes.

Though we must admit that the question cannot at present be answered, we need not regard the subject with any great alarm, or believe that the mortality from immaturity alone is excessive beyond the requirements of nature. It has been pointed out already that this mortality, exclusive of congenital defects and birth-injuries, amounts to 26 per 1000 births-i.e., 2.6 per cent. Should we regard as excessive a figure which shows that $97 \cdot 4$ per cent. of all infants who are not still-born are vigorous enough to overcome the very severe strain and the fundamental organic changes implied in the assumption of a separate and independent existence? To no analogous condition in later life can we compare the risks of birth, but the only occasions on which a sudden and severe strain is thrown upon life is in acute diseases, and few such levy so light a toll as 2.6 per cent. We must further remember that a child is inevitably exposed to certain dangers during the process of birth. With medical assistance these can generally be forestalled, but often this help is not at hand. Among the poor, confinements are usually managed by midwives, many of 
whom are totally unqualified to recognise, still less to avert, the difficulties that are likely to present themselves. To this circumstance we must ascribe some of the deaths that are attributed to immaturity.

Though we are thus led to take a less pessimistic view than is customary at the present time, a grave matter requires mention before we conclude this subject. In the last chapter the mortality of legitimate and illegitimate infants showed how large is the share of deliberate parental neglect in raising the mortality returns. This disclosure makes it necessary to ask whether the deaths of any of the 2.6 per cent. of immature infants are accelerated or even directly due to a similar cause, and whether these should be attributed to immaturity at all. It is well known that a feature of modern life is the desire to avoid parental responsibility. The wide-spread practice that has grown up in order to carry this desire into effect is associated with devices which are reprehensible, physically as well as morally, and have for their object the premature termination by artificial means of the natural process that follows conception. These facts need not be dwelt on further here than to draw the conclusion that there are those who, in their determination to evade parental cares, will adopt measures which are not only abhorrent to morality and offensive to law, but injurious to the individual. Finally, the trade of "baby-farming," which flourishes all over the country, shows that the callousness disclosed by the abovementioned practices survives even at the time when the maternal instinct should be strongest.

The bearing of these considerations on the mortality from immaturity is clear. If we admit, as we must, that to many the value of the life of an unborn child is nothing; and if we admit, as we must, that deliberate neglect is allowed to pile up a heavy mortality during the first year of life;-then we must admit that the callousness to infant life will be no less during those first few days or weeks, when the new responsibility will be felt most acutely and tolerated only with impatience. A child is never so helplessly at the mercy of others as during the few minutes when it is making its entry into the world. At no 
other time from conception onwards is it so easy to interfere with vital functions either by active measures or by the no less efficacious method of neglect. During the hours and days immediately following birth, deaths are so common as rarely to awaken suspicion, and a frail life that is clinging to existence with the feeblest of grasps needs only a touch to set it adrift. Considerations such as these warn us to be careful before we accept immaturity as a cause for the deaths of so many newborn children, and make us hesitate before we conclude that none of these lives could have been saved with ordinary precautions. That these fears are not groundless is proved by the fact that it has been found necessary to introduce a bill into Parliament, ${ }^{1}$ by which the destruction of a child's life during birth will be made a felony punishable by penal servitude for life.

\section{Conditions of LATER InFANT LifE}

Infants that have survived the first four weeks after their birth should have put behind them the most dangerous period of their lives. Henceforth their existences should be, and in most cases would be plain-sailing, were they allowed to steer their course by the light of modern science. Unfortunately the conditions under which most of them are brought up lead to a further and even greater loss of life than before.

One of the most mournful reflections on these lamentable conditions is that they could be improved and their results prevented by methods which are of the simplest. The requirements of an infant are so primitive that, were they fully appreciated, almost every mother, no matter how poor, might have at her disposal the means of satisfying them, and no matter how unintelligent, might know how to meet them. Every infant born untainted by disease could have his life practically assured to him by the observance of two conditions-proper feeding and scrupulous cleanliness. Were these facts better recognised, sickness and death in the last eleven months of infancy would dwindle to relatively unimportant dimensions.

1 Infant Life Protection Act, 1909. 
The trivial value that is set by the lowest classes on infant life must be witnessed, and witnessed often, to be fully appreciated. Extreme poverty, though it seems reckless in the matter of large families, shows its mercilessness when the new mouth begins to cry for the food which cannot be spared. Maternal instinct is converted into selfishness, and the only being to whom the infant can turn ceases to trouble for its welfare. Often the worst degrees of poverty are associated with drunken habits, and the child meets with nothing but neglect. Such victims are not taken to a hospital until the end is near, and even then the motive is not the child's welfare, but rather the need for a death-certificate in order to realise an assurance policy. These cases, seen once or perhaps twice before death, are often a cause of anxiety to hospital medical officers; for though no reasonable doubt may exist that death has been accelerated if not caused by neglect, legal proof is almost impossible to obtain, and so punishment is difficult.

Yet indifference and neglect will often act just as fatally when instigated by motives other than those of poverty or life assurance. We have already noted the effect of illegitimacy. Closely associated with this question is that of baby-farming. For reasons which are often though not invariably connected with illegitimacy, many mothers of the servant and other classes surrender their new-born infants to the entire charge of women who, for a money payment, undertake the maternal duties. It is a common arrangement for the stipulated payment to be made in a lump sum at the outset; in other cases, periodic contributions are made for a while, and then arrears accumulate. In either event, the pecuniary interest of the hired mother and the continued welfare of the nursling do not coincide. So notorious became the abuses of this system, that the Infant Life Protection Act was passed in 1897 , to make obligatory the registration of all houses receiving for hire or reward more than one infant during a period of more than twenty-four hours. Whatever the effect of this Act has been, it has fallen short of the hopes which accompanied its enactment. The harmful influence of women who make their livelihood by accepting the care of strange infants is still being widely exercised, despite the 
exemplary infliction of the capital punishment on four offenders within the past few years. Recently, however, this question has been further dealt with in the Children Act, I908. Section I. of this Act provides that all persons who undertake for reward the nursing and maintenance of one or more infants shall give notice of this fact within forty-eight hours to the local authority. By this means, all those instances in which only one child is received at a time, and which therefore escaped registration under the Act of I 897 , are now brought under supervision. As an additional assurance that these infants shall be properly cared for, the local authority is instructed to appoint "infant protection visitors." Their duty is to visit the infants and the premises on which they are kept, to satisfy themselves as to their proper nursing and maintenance, and to give any necessary advice.

The suffering and loss of life which result from neglect at the hands of baby-farmers are wholly criminal; but numerous infants endure the miseries of neglect, perhaps to a smaller degree, from a cause which makes it difficult to blame the parent. Few stories are more often heard in the outpatient rooms of a children's hospital than that of a mother from the surrounding slums who has of necessity to be away at work from morning till evening earning enough to keep the home together, while her infant is left in the care of its sisterherself but a child-or more often of a neighbour, who sells her grudging services for a few pence. Sometimes the baby is left by the mother at a neighbouring crêche on her way to work in the morning, and taken away on her return in the evening. Occasionally it may be learnt that the mother devotes part of her dinner-hour to suckling her infant, but most frequently the child is in hired hands from about 8 A.M. to 6 P.M., and can obtain only artificial food. These crêches carry on their work with varying success; and though some are well managed, others show small consideration for their charges. A mother circumstanced like this is often poles removed from those whose neglect is deliberate, and, with the heaviness of despair, she will watch her child ailing day by day. Yet often the illness has far advanced before she can afford the loss of a half-day's 
work to bring the patient to the hospital. These cases raise the question of the industrial employment of married women in the period immediately following their confinements, but the discussion of this subject will be deferred until we deal with the remedies.

Dirt-Bad Feeding:-The two most serious dangers in an infant's simple surroundings are uncleanliness and improper feeding. Each is a fertile source of disease, but they are so often intimately associated in action that it is not always easy to trace their respective influences on any particular victim. That uncleanliness forms the lot of the majority of infants will be denied by no one familiar with the conditions of the poor, and it is equally certain that it is more prevalent in towns than in country districts. We have already had occasion to notice the excessive mortality in urban as compared with rural districts, and we have noted the greater prevalence of sickness in the former. The insanitary state of large numbers of the poorer quarters of towns is responsible for much disease. Their harmful effect is strengthened by the uncleanliness of the living-rooms themselves, and, to complete the sinister catalogue, the personal hygiene of the infant is often almost wholly neglected. The virtues of washing and of clean garments are unknown, and diseases, parasitic and other, are the frequent results. ${ }^{1}$ It is difficult to estimate the extent to which the lung-diseases of infancy are attributable to dirty surroundings, but it is probable that there is some intimate connection. The accumulation of dust and dirt provides in addition a source of infection for tuberculous diseases. Infants who are left to amuse themselves on the floor, stir up the dust into the air they are breathing, and contaminate the fingers which they suck at frequent intervals. Another danger of

1 The apathy shown by many women of the poorer classes to the personal cleanliness of their babies is often illustrated in a children's hospital. The late Sister in charge of the Out-patient Department of the Evelina Hospital tells me that more than once she has witnessed the following incident, the humour of which is lost in its pathos. An infant has been brought to the hospital for "wasting " of some weeks' duration, and has been undressed for medical examination. At the sight of its shrivelled body the mother has begun to cry, and has explained her emotion with the ingenuous admission that her baby was a beautiful child when last it was undressed. 
infection is encountered in tuberculous relatives, especially such as live in intimate daily association with an infant, and perhaps share with it the one room that serves for living and sleeping alike.

These, in brief, are the principal risks from uncleanliness. Of no less importance is improper feeding. Food may be improper in one of two ways. It may be deficient in some chemical constituent essential to health, or it may be compounded of the proper ingredients in the right proportion, and yet contain in addition some deleterious substance. Both these faults are very common. Food that is improper by deficiency of nutritive value necessarily results in starvation, marasmus, or rickets. Human milk itself may be thus deficient. The fault is either one of quantity or of quality, but in each case the needs of the infant go unsatisfied, and various degrees of starvation result. Occasionally rickets appears in infants who have been regularly fed by the breast, and in these the disease is attributable to the lack of some essential constituent in the human milk, the composition of which is variable. The breast-fed infant, however, is relatively safe from all these ill-effects compared with the child reared on the bottle. Many cases of nutritional disorders arise in children whose only diet is certain condensed milks and infants' foods.

The most serious harm, however, is effected by food that is improper by reason of some deleterious and adventitious constituent. Cow's milk, as given to infants, only too often comes under this suspicion, and is responsible for a large proportion of the illnesses of the first year. The two diseases that are especially associated with its use are infantile diarrhoea and tuberculosis; and, since each of these is often fatal, it will be understood how prominently they must figure among the causes of death. Clinically, too, they are among the most frequent causes of sickness. The special conditions on which they depend must therefore be considered in detail.

Infantile Diarrhea.-The essential features of this disease can be easily summarised. Its greatest activity is limited to the few hot months of the summer. It specially affects the last three trimesters of infancy. It is a disease of towns rather 
than of the country. Its incursions are heaviest where the surroundings are most squalid. It marks down the bottle-fed baby and spares the breast-fed.

Few cases are to be seen in May and June. In July they are more frequent, and in August and September they overcrowd the out-patient department of the children's hospitals. Towards the end of September the number of cases diminishes, and from October till the following May few if any examples are met with. Every year the same sequence is observed, until a cold summer (as in 1907) appears, and the condition becomes relatively infrequent. A comparison of the seasonal mortality with the mean atmospheric temperature has been found to show the favourable influence of warmth on the causative agent of the disease.

The next point is that few of the patients are under three months of age. Of 22 I consecutive cases seen at the Evelina Hospital, 33 were under three months, I 88 were above that age. This relative immunity of the youngest infants is a suggestive fact, though its importance is masked in mortality returns, which give the figures from diarrhœa in each trimester as 8.82, I 1.04, $7 \cdot 63$, and 5.14 (1906)-i.e., a higher mortality in the first trimester than in any other except the second. These figures, however, express the greater susceptibility of very young infants to the disease.

Let us here note another important fact-namely, the personal condition of the patients and of their mothers. The children are frequently among the most neglected-their bodies dirty, their clothing malodorous-and the mothers are among the poorest who visit the hospital. If the observer will take the trouble to follow some of these cases to their homes, he will find that they come from the most unwholesome courts and allies of the neighbourhood, and he will form the opinion for himself that squalid surroundings play their part in inducing the disease. If statistics were available, to allow a comparison between the incidence of the disease and the condition of the dwellings, we might confidently anticipate a confirmation of this belief. The following statistics, which show the mortality from diarrhœa in urban and in rural 
districts respectively, clearly indicate the influence of town surroundings :-

Average Mortality from Diarrha. Male and Female Infants.

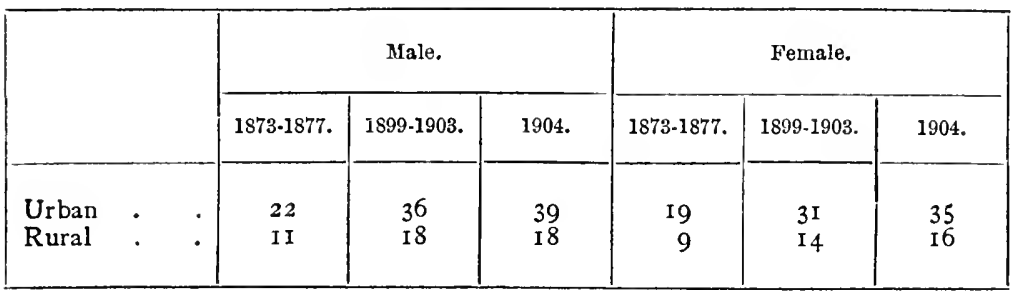

The mortality is thus seen to be twice as heavy in urban as in rural districts.

If we enquire as to the method of feeding in each case up to the time of the onset of the symptoms, we shall find that the artificially-fed babies are attacked, while the breast-fed are relatively spared. This is well exemplified in hospital practice. During the five weeks from I 5 th August to 2oth September 1908, 221 cases of summer diarrhœa were brought to the Evelina Hospital, and in each case a note was kindly made for me by the Resident Medical Officers as to the nature of the feeding. Of these $22 \mathrm{I}$ cases, 203 were under eighteen months. Of the latter number, only $4 \mathrm{I}$ were stated to be breast-fed, while the remainder, 162 , were hand-fed. In other words, 4 out of every 5 infants were hand-fed.

Dr Niven pointed out in $1896^{1}$ the greater incidence of diarrhoa in hand-fed infants, and Dr Newman in Finsbury, and $\operatorname{Dr}$ Newsholme in Brighton, have shown ${ }^{2}$ that the percentage in breast-fed infants may be even less than 20. We have already noted that infant diarrhœa is least prevalent in the first few months of life. At this period, more infants are kept at the breast than at any other time.

The foregoing considerations clearly indicate that a special danger attaches to artificial feeding. On the other hand, it is

1 Lancet, I896, vol. i., p. 535.

2 Infant Mortality, G. Newman, M.D., 1906. 
well known that infants can be safely reared on the bottle, provided every care is taken to secure pure milk, and to give it fresh and in clean vessels. We must conclude that it is not the milk itself, but the contamination of the milk, that effects the harm. In this fact lies the explanation of the special association of the disease with summer when bacterial organisms flourish, with towns, with squalid surroundings, and with unclean persons. The question at once arises, whether the contamination takes place in the dairy-farm, the milk-shop, or the dwelling-house. If it occurs at any stage before the milk passes from retailer to customer, summer diarrhœa may be expected to be less an infliction on any one class of consumer than on any other-for epidemics of, for example, scarlet fever arising from an infected milk-supply, take no heed of social distinctions-but we know that it is the lowest class that suffers. On the same assumption, we would expect that infants fed on condensed milk would escape, but they are victimised with those reared on cow's milk. Lastly, some cases-few, no doubt, but too large in number to be neglected-occur in breastfed babies. The conclusion is that the source of contamination must be sought in the dwelling-place of the consumer. This view does not of course exclude, the possibility of infection before retailing, but in the large number of examples the infection is a domestic one.

No specific germ has been identified as the cause of this, and it is possible that more than one variety may be concerned; but our ignorance on this point does not hinder us from taking the right measures of prevention. These are clean receptacles for the morning and evening supplies of milk; air-tight lids to prevent absorption of atmospheric impurities or pollution by flies or dust; clean feeding-bottles; and finally, the abolition of the rubber "comforter," which becomes crusted at its base with soured milk and picks up dirt each time it falls to the floor. If, in addition to these precautions, the milk is boiled on delivery, we shall have gone far to rid infancy of its greatest scourge.

Tuberculous Disease.-Tuberculous diseases are among the most frequent causes of infant death. Reasons have already been given for the belief that the mortality returns for these 
diseases (4.92 in 1906 and 4.54 in 1907) are underestimates of these deaths and-since infantile tubercle is usually fatal-of the incidence of the condition. Further, tubercle, unlike pneumonia or diarrhœe, is not a disease that kills within a few days of its onset. It runs its course for weeks or months after the initial infection. As we have seen, some of the deaths, therefore, that occur in the second year, will be the result of infection arising within the limits of infancy. Allowance must be made for this fact in appraising the importance of tubercle as a factor in infant health. This is the more necessary, seeing that an infant may be infected for many weeks and may even die before revealing definite symptoms of the disease; and therefore many such patients may not figure in the mortality returns from tubercle.

The next question is the channels of infection. The risks of dirt and of tuberculous relatives have already been instanced, and we have now to consider infection by food-i.e., cow's milk. The distinction between human and bovine tubercle drawn by Professor Koch in 1901 at the International Congress on Tuberculosis has been disproved by the second report of the Royal Commission on Tuberculosis of 1907 ; and in cow's milk we must recognise one of the sources of human tuberculosis. The importance of this cause depends on the frequency with which milk is contaminated. Bacteriological examination has shown only too conclusively the frequent presence of active tubercle bacilli in milk-supplies. Thus Drs Niven and Délapine examined 93 samples of milk arriving in Manchester, and found that 18 of them contained tubercle bacilli. If so large a proportion of tuberculous milk is sent into a city where a strict inspection of milk is enforced, the proportion is not likely to be smaller in London and other large towns, where the public health authorities are allowed less power to control the milk supply. ${ }^{1}$

The matter may be concluded by a brief reference to post-

1 Under its General Powers Act, 1907, the London County Council arranged last year for the bacteriological examination of samples of milk brought into London. Of 92 samples of milk conveyed by four of the chief railway companies, no fewer than 22 proved to be tuberculous. 
mortem records. Of Ioo children under two years who died from tubercle in the Hospital for Sick Children, Great Ormond Street, 22 showed evidence of infection through the intestine. ${ }^{I}$ Of 300 cases of abdominal tubercle recorded by $\mathrm{Dr}$ Nathan Raw ${ }^{2}$ not one was breast-fed.

\section{TIIE REMEDIES}

Though infancy must ever remain one of the two most critical periods of life, it could without insuperable difficulty be released from much of the disease which literally decimates it at the present time. Many, if not most of the causes of the deaths are preventable, others are partly preventable, and only a few are beyond the control of modern science. The fatal conditions have been traced in the foregoing pages, and hardly one of the more prominent does not suggest its own remedy. What are they? And are they at our disposal ?

Infant mortality, and with it infant disease, must be attacked from two directions-the legal and the social. It must be controlled by laws which impose deterrent punishments on those who hold the life of a child cheap and who actively or by deliberate neglect compass its death. In this way we shall reach the baby-farmer, and all those who for money gain are directly or indirectly responsible for adding to the high deathrate of illegitimate and legitimate children. By legal help infants can be protected from the dairyman and milkman who trade in milk that is polluted. Yet another way in which the law can assist is by giving to infants, if only for a few weeks, the right which every farmer allows in full to his calves and foals - the right to their mother's care and milk. The mere existence, however, of these laws on the statute-book will not save a single life: they must be made operative.

But though the law can effect much, it cannot do all or nearly all. The evil must be approached from a second standpoint, which is even more important and promises greater results. Ignorance of the needs of infancy must be driven out

1 Diseases of Children, Goodhart and Still, 8th ed., I905.

${ }^{2}$ British Medical Journal, vol. ii., 1904. 
from the homes of the poor. When this has been done, a proper regard for infant life will take its place. This change is the fundamental one, but it will not be effected solely by measures aimed directly at infant mortality. We must recognise this subject as one aspect of a vast social question - that of the poor-and not until this has been adjusted will the lesser difficulty be overcome. If it were possible tomorrow for each infant to find its home clean and healthy, the problem of the poor would have been solved: conversely, if the problem of the poor were solved, excessive infant mortality would cease to trouble us.

We can no doubt indicate readily enough what is wrong, and we can even more readily suggest how the defects should be made good, yet no one but a visionary can persuade himself that the practical application of the cure is either easy or likely to be completed for many years to come. Inasmuch as the condition of the infant population depends on that of the adult population, we must be prepared for the occurrence of preventable sickness and death, despite the fact that science has already the means of lessening both. The recognition of this limitation is, however, no reason for disappointment, nor is it any excuse for inaction. A knowledge of the dangers of dirt and bad feeding should be spread among those who are most ignorant, and measures should be pressed forward to make their surroundings more hygienic. The harmful conditions have been traced, the remedies are known: the imperative necessity is to rouse those who have it in their power to organise the requisite resources.

We must take as our inspiration the knowledge that the majority of mothers, no matter how poor, are genuinely solicitous of their children's good. They are anxious to do well by them so far as their circumstances permit, though the overwhelming number do not recognise the road that leads to death. They are profoundly ignorant on the subject which is more important to them than any other, but they are ready to learn, and the hope of the future lies in their instruction.

Lessons on baby-rearing can be given even to school-girls; but fruitful results will be obtained from instruction, com- 
bined with advice and supervision in its practical application, to young women-especially those who possess or shortly will possess babies of their own. Within the last few years many associations have been formed with this object, and though the movement is only in its beginnings, the results have already proved its value. The lines which this work should follow are by now fairly clearly recognised, and the following particulars of a working plan will show those who are interested in the matter how the instruction and supervision can best be given. The details represent the scheme successfully put into practice by the Westminster Health Society-a pioneer body which has served as a model to younger societies in different parts of the country.

The society, which is a voluntary one, works in close cooperation with the public health authority and with the charitable and social agencies in the city. In addition to preparing and distributing leaflets on such subjects as infant management, hints to mothers, summer diarrhœa, measles, and whooping-cough, it arranges for frequent popular lectures to mothers on food, home-nursing, children's ailments, and infectious diseases. Of even greater practical value is the system of house-visiting, which constitutes the main object of the society. A staff of voluntary lady visitors, trained to the work and chosen for their tact, patience, and perseverance, are engaged in visiting homes where there are infants. A special district is allotted to each visitor, and from time to time the staff meet to exchange experiences or to listen to lectures dealing with their daily work. An attempt is made to begin the visiting even before a child is born, and, with the help of various hospital authorities, a list of impending maternity cases is prepared. A call is paid to each address some little time before the confinement is expected. Should the environment be found unsatisfactory, additional visits are arranged in order to improve the condition of the home. Extra nourishment is provided if necessary at the time of the confinement, and facilities are given for mothers who wish to save money for their lying-in. By this means an attempt is made to ensure that the home is in a fit state before the baby is born, and that 
the mother understands the requirements of her infant, and how to satisfy them.

The cases visited after delivery include, in addition to the above, all those heard of through official registration. These are at once visited, and the mother receives proper advice. The calls are repeated at intervals depending on the requirements of each case, but are made at least once a month for the first three months, and thereafter once a quarter. Complete records are kept of each case, and should any infant begin to ail, it is sent, with an explanatory note from the visitor, for medical advice.

The practical result of this undertaking has been to establish a very satisfactory difference between the infant mortality in the visited homes and that in the city generally.

It is clear that a system, such as the above, is founded on the principle that infant mortality is a domestic problem and that improved conditions of life are best secured by direct personal appeal to individual mothers. This truth is bound to receive wider recognition in the next few years, and its practical application is likely to be made by voluntary or municipal enterprise in every neighbourhood where the mortality is unnecessarily high. Yet even these measures will not suffice if two other matters remain unimproved. The first is the milksupply, and the second is the care of those infants whose mothers go out to work.

A few municipal authorities have already taken action for the supply of pure milk, and depôts, planned on similar lines to the "gouttes de lait" of France and Belgium, have been established at St Helens, Battersea, Lambeth, Woolwich, Bradford, Burnley, Liverpool, and Ashton-under-Lyne. Although these institutions have not yet succeeded in producing as great an effect on local infant mortality as was generally hoped, ${ }^{1}$ they indicate a step in a forward direction. Seeing that infant mortality is fundamentally a question of the home, we can easily understand why these depôts have not been more successful. It is the slovenly, ignorant mother who is the

1 Indeed, one leading city (Birmingham), has decided against their usefulness. 
greatest danger to an infant, yet it is just this type of woman who will not make an effort to avail herself of the benefits of a pure milk-supply. The milk, prepared under every scientific precaution at these depôts, is incomparably better fitted for infants than that retailed at the average milkshop; but the practical difficulty is to arrange that it shall be used in the homes where it is most needed. Even in cases where this end is attained, the risk is always present that the good effect of the careful preparation will be undone by dirty and unhygienic domestic habits.

The second difficulty which has yet to be overcome by State or municipal action is the provision of proper care for those infants whose mothers are working all day away from home. The entire dependence of an infant on others, and the difficultywhich, among the poor, often amounts to an impossibility-of obtaining proper attention for it in its mother's absence, combine to emphasise the importance of placing the care of all babies with their mothers. Though this arrangement is desirable throughout the whole of infancy, it is only a counsel of perfection; but in the earliest and most critical weeks of life, it should be possible to secure for a baby this amount of attention. The legislatures of most European countries have wisely appreciated its value, and in many states, including Hungary, Austria, Holland, Belgium, Germany, Portugal, and Norway, ${ }^{1}$ a period of four weeks' rest after confinement is made obligatory on women who are industrially employed. Though the credit of taking the lead in this matter in modern times lies with Switzerland, where a rest of at least six weeks after confinement has been enforced since 1877 , the first enactment on the matter appears to have been made as long ago as 1755 , when a Swedish edict imposed a money fine on any mother who, by neglecting to suckle her child for at least six months, caused its death. ${ }^{2}$

In England, the law prescribes a four-weeks' rest after confinement, but the obligation is easily evaded, and in practice the law has proved a complete failure. It is to the interest of

1 The Infant, the Parent, and the State, H. L. Heath, 1907.

2 Infant Mortality, G. Newman, M.D. 
the employer to regain the services of his "hand" as soon as possible, and it is important to the mother that she should resume her wage-earning without delay. Between these two the welfare of the infant is forced into the background. The difficulty is not one that allows a ready solution. The prosperity of the child demands not only that the law should be made operative, but that the time-limit should be extended to at least twice its present length; but the mothers whom this alteration would affect are not those who can afford to spend some months out of work, especially at a period when a new baby has brought additional expenses to the household. The employer will not be willing to keep open a situation for so long. If a period of abstention from work is to be enforced, the wages thereby lost to the mother must be made good. In Germany the difficulty is overcome by the system of compulsory sick insurance; in Spain the cost falls on the employer; while in other countries systems of partial insurance and of daily subsidies are adopted. The subject is one that cannot be indefinitely shirked in this country, and a recent move in the matter by the Local Government Board shows that it is receiving attention, but it will not be solved until public opinion recognises that a woman who brings a child into the world has done the State a service, and that it is for the State to see that the young life is given a fair chance at its outset.

For somewhat older children a properly organised system of day-nurseries, subject to medical inspection and supervision, is urgently required. Infants and young children under school age can thus be tended and fed throughout the day for a reasonable charge among healthy surroundings. $U_{p}$ to the present time crêches have been in most cases private undertakings, and they by no means always attain a satisfactory standard either by way of food, cleanliness, or attention, and their arrangements often invite the spread of infectious disease. In England we are far behind many other countries in this matter. ${ }^{1}$

1 The National Society of Day Nurseries was founded in 1906 to do. away with this reproach. Its object is to open model day-nurseries, and to provide a central authority to raise the standard of crêches throughout the United Kingdom by affiliating them to the society. 
In Sweden crêches have been organised for a great many years and are entirely self-supporting. In France the first crêche was founded over sixty years ago, and at the present time there are some five hundred, each of which is under Government inspection, and no new crêche can be opened without the authority of the Prefect.

It should not be difficult to establish suitable nurseries in this country. They are essential in every district where women's labour is in great demand; though in order to prevent their improper use, each mother, before being allowed to avail herself of their benefits, should satisfactorily prove that she goes out to work of necessity. They should be healthy in every respect, and a source of pleasure and profitable training to older children. Such institutions might be made centres for teaching mothers, and the influence they would thus exert would provide yet another means of leavening the ignorance of the poorer classes, and of relegating their present-day methods of infant-rearing to the traditions of a dark age. 


\section{CHAPTER XII \\ CHILDREN AND DISEASE}

InCIDENCE of Disease in CHILDHoOd: Factors diminishing the Incidence of Disease in Childhood; The Simplicity of Child-life; Secondary Pathological Changes ; Hereditary Diseases ; Sexual Instinct ; Diseases incident to Parturition; Habits of Excess; Occupational Diseases ; Muscular Strain; Many Diseases of unknown Pathology restricted to Adult Life-Factors increasing the incidence of Disease in Childhood; Congenital Deformities; Injuries at Birth; Susceptibility to Bacterial Infections; Interference with Growth; Physiological Equilibrium of Childhood unstable; Certain Diseases restricted to Childhood; The Helplessness of the Young. PECUliarities of CHILdren in DiSEASE: Children dislike Illness; Neurotic Affections uncommon; Malingering; Chronic Illness; Loss of Muscular Activity; Mental Tiredness ; Habit-spasms ; Interference with Growth ; Heart-disease ; Appetite ; Acute Illness ; Constitutional Symptoms prominent ; Epileptic Convulsions ; Cerebral Symptoms ; Irregular Clinical Course ; Rapidity of Recovery; Complications and Sequelæ frequent; Sudden Death.

\section{The Incidence of Disease in Childhood}

WHILE each successive period of life is marked by an increased liability to some diseases and an exemption from others, the two ages at which health is in most danger are the beginning and the end of life. A curve planned to represent the incidence of disease at all ages would have its greatest height in the first year; it would then fall, at first rapidly and then more gradually, to about the eighth year, when it would rise slowly until the eighteenth year. From this time it would again decline to a minimum between the twenty-fourth and thirtieth years, after which a steady rise would begin, continuing into old age.

There are many causes to account for this heavier incidence in early life than at any later period, even that of old age, though 
numerous circumstances of child life must favourably affect the liability of the young to disease. Each of these two groups of influences deserves special attention, and consideration will first be given to the factors that work for the benefit and protection of the young.

Factors diminishing the Incidence of Disease in Childhood.At the outset we must note the relatively simple daily life of young children. We know that the effects of disease are an expression of the reactions of the tissues to altered environment, and that the causative agents of disease are being increasingly found rather in the surroundings of the individual than in his own organs. The more complex and changing the former, the greater must be the chances of encounter with pathogenic conditions. The daily round of the average adult brings him into contact with innumerable possible sources of danger. The apparel of the crowd that jostles him in the street: the intimate propinquity, in office, shop, or public conveyance, of his fellows, many of whom must certainly carry with them the seeds of disease: his meals, some taken in public eating-places, where the food is handled before reaching his plate by numerous workers of dubious cleanliness-all these must add to his risks of acquiring disease. The customary environment of childhood, especially in its earlier years, is relatively exempt from these risks. The child of a careful mother, fed with her own breastmilk, or with milk prepared to reduce the chance of infection to a minimum, is kept clean by frequent baths, and in his excursions into public places is well protected from undesirable contact. Only later, when he has passed the age when he can be thus conveniently shielded from harm, do his risks begin to increase, until, with the advent of the day when he first mixes with others in classroom and playground, his liability to disease begins to show a steady rise.

Next, the fact is important that a large proportion of the pathological conditions of adults are the secondary results of pre-existing disease-for example, heart-disease due to chronic bronchitis, or cancer to an earlier ulceration. But those children who are born free from inherited disease, have no previous medical history; and therefore no secondary changes 
can be suspected until the primary condition has been acquired and has had time to produce its later results. Similarly, many hereditary conditions which tend to affect the members of successive generations of a family, do not disclose themselves until adult life. As common examples of these, we may cite arterio-sclerosis and certain mental affections.

Again, numerous circumstances incidental to adult life but absent in earlier years are responsible for much illness. Foremost among these must be placed the sexual instinct, which remains a prolific cause of adult disease. In childhood the changes accompanying acquired syphilis are hardly to be looked for, and therefore the long train of subsequent degenerations rarely occurs. Thus endarteritis, aneurisms, and thrombosis, resulting from this infection are very uncommon. The formidable list of ills associated with menstruation, gestation, and parturition are necessarily absent. Morbid states, the results of habits of excess, are exceptional until after adolescence. The various effects of alcoholism-such as cirrhosis of the liver or peripheral nephritis-and of drugtaking are little more than curiosities of juvenile pathology. The numerous diseases of adult life that are occupational in origin develop only rarely among the young. The only analogous conditions that occur in early life are those referable to the one occupation of childhood-namely, schooling. These we have dealt with in a previous chapter. Another circumstance to be noted is the absence from childhood of those conditions which, in men, are usually ascribed to excessive muscular or nervous strain, such as aneurisms of the larger arteries, simple dilation of the heart, and neurasthenia.

Finally, certain diseases are prevalent only among adults, though their pathogeny remains too obscure to allow any satisfactory explanation of the immunity of the young. Gout and osteo-arthritis, primary chronic Bright's disease, emphysema, gastric ulcer, pernicious anæmia, chlorosis, cancer, exophthalmic goitre, Addison's disease, and acne are some of the affections that are little seen in children. In addition there are the affections of a chronic degenerative character which are usually associated with declining age-such as 
atheroma, senile gangrene, paralysis agitans-and most of the chronic lesions of the brain and spinal cord.

Factors increasing the Incidence of Disease in Childhood.-The above factors must clearly lighten childhood of a very great amount of disease. On the other hand, we have to set against them an important series of influences which are directly responsible for the great incidence of disease in the young.

First in point of time come maldevelopment and obstructed labour. These have already been discussed as affecting infancy; but in many cases the more serious results may be deferred until later periods-five years, ten years, or even later. Thus children with congenital heart-disease not infrequently live for some few years after infancy.

Another cause, broader in its application than the foregoing, is the absence in early childhood of the protection afforded by acquired immunity. Many diseases bequeath an exemption from further attack which endures throughout life, but the young child brought into contact with these infections for the first time is taken unprepared, and can offer no resistance. Throughout his earliest years, the care and watchfulness of his parents may enable him to escape many diseases. With the advent of schooling, however, and the unavoidable intercourse with other equally susceptible children, the opportunities for infection multiply. The ensuing few years will therefore be punctuated by the appearance of many of the specific fevers. Not only does the prevalence of the infective fevers in early life and the acquired immunity of the many who survive to adult age unite in increasing the relative incidence of disease in childhood, but the very large number of after-results of these affections operates still further in the same direction. This is especially the case with such common conditions as measles and whooping-cough. They are widely regarded as comparatively harmless complaints, in spite of the fact that they leave behind them a long trail of serious effects.

In addition to the specific fevers, other diseases, also bacterial in origin, are peculiar to childhood or attain their maximum incidence at that period. Infantile paralysis is of frequent occurrence in the first three years, but it is not known 
in adult life. Rheumatic fever and chorea, though prevalent well into mature age, are especially diseases of children above five or six years. Posterior basal meningitis attacks the young in their first year, and tuberculous meningitis is found most usually in subjects between two and five years old. Impetigo, ringworm of the scalp, erythema nodosum, and lichen urticatus are some of the common diseases of the skin that specially affect children. Enlarged tonsils and adenoids are essentially juvenile conditions.

Next, we must recognise that a fundamental condition which confers a distinctive character on the pathological history of childhood is the presence of active growth. This process is readily susceptible to morbid influences. It provides yet another explanation of the frequency of ill-health in the young, in whom nutritional disorders are among the commonest. Development may be interfered with as a whole, as in marasmus among infants and in the wasting that follows many acute illnesses in older children. On other occasions merely a function or a tissue has its growth arrested. As instances of the arrested or delayed growth of the former, we may mention acquired idiocy and the more trivial condition of incontinence of urine produced by a backward development of the nervous centres controlling the movements of the bladder. The tissue that suffers most commonly from interrupted growth is the skeleton. This is specially vulnerable, owing to the fact that the bones during infancy are structurally more backward in their development than other tissues. In rickets we have a condition which by its nature presupposes active growthnamely, that of cartilage changing to bone. This disease is therefore peculiar to childhood. Another example is scurvy. Though this affects the mature as well as the young, and is for the most part similar in its effects at all ages, even to the effusion, in severe cases, of blood beneath the periosteum, it presents in earlier years a result that is never observed later. The growing epiphyses of one or more long bones often become separated from the diaphyses. A similar distinction holds in regard to syphilis, which in its congenital form attacks the susceptible extremities of bones such as the 
femur or humerus, but cannot produce these effects in adult bone.

By reason of their rapid growth, children are in a state of physiological unrest and change. Even weak forces may therefore impress or deflect the course of their health. Every such abnormal modification may itself be the starting-point of a chain of results each more serious than the last. Consequently many minor conditions which would easily be thrown off from the more stable constitution of an adult may lead to extensive changes in the young. Examples of this are in the experience of everyone. Thus a young girl was permitted to wear an elastic belt to afford support to her stomach during paroxysms of coughing. This practice was continued even after the cough had ceased. At the end of a year her ribs had become so crumpled and her chest so deformed by the continued pressure, that both her breathing and circulation were seriously impaired. Her general health became undermined, and she is now condemned to an invalid life, lacking the energy to join in the games or interests of her companions. Again, we know that the loss, by injudicious extraction, of teeth from a child's developing jaw may so modify the growth of the bone as to produce deformity in later years. Not uncommonly a young child, when convalescing from an attack of some such fever as measles or even influenza, shows symptoms of paralysis of one side; henceforth the intelligence ceases to develop, and a state of incurable imbecility may gradually supervene.

In yet another group of diseases specially attached to childhood, we have certain affections of the motor apparatus. Though they are not common, and can therefore take only a relatively unimportant part in swelling the incidence of juvenile disease, they must no doubt depend on some factor belonging to early life. Friedreich's ataxy is peculiar in that it begins a few years after birth and often affects several members of a family. Pseudo-hypertrophic paralysis is a disease of childhood, as also are certain juvenile forms of muscular atrophy (Duchenne's infantile type, etc.).

Probably the most important of the factors that increase the amount of disease in childhood is one that has already been 
fully dealt with in the chapter on the Conditions of Infant Lifenamely, the helplessness of the young and their more or less complete dependence on the offices of others. Whereas instinct or experience provides animals, both parents and offspring, with a shrewd knowledge of what is harmful, the intelligence of children is for years too rudimentary to be of this service; and even should an infant display indications of distaste or discomfort, these actions are often misinterpreted or ignored. Experience shows even among the better classes of society a considerable ignorance of the simple requirements of child-life. Among the lower classes this ignorance is distressing and almost mediæval. The most elementary precautions-some so simple as to be observed even by animals themselves-are neglected and their place filled by observances which only too often invite disease or even death. The question of food and cleanliness will not be further considered than to observe that the absence of these two essentials is a greater factor than any other in producing ill-health, and that therefore on these rest the chief responsibility for the excessive incidence of disease in childhood. All the commonest affections of this age that are not the direct result of improper feeding, either have already been proved or are likely to be proved to be due to micro-organisms. Between a comparatively innocuous affection like thrush-the causative fungus of which would have no opportunity to grow were the child's mouth kept clean-and a deadly one such as tetanusresulting from access of dirt to the navel of a new-born infantthere is a whole catalogue of diseases due directly or indirectly to uncleanliness.

\section{Peculiarities of Cimldren in Disease}

With few exceptions the pathological processes of youth and of maturity are alike. Their clinical effects, however, are different at the two ages, and many of the obstacles encountered in dealing with sick children are due to the peculiar reactions of the young to disease. The familiar landmarks which are the A.B.C. of diagnosis in adults, are, in children, either absent, or, what is more confusing, are shifted in position. The learner finds himself a wanderer in a strange land, where signs are 
written in an unknown script, and his bewilderment is none the less because many of the scenes appear familiar, and yet on closer inspection prove in some imperfectly comprehended way to be only vaguely recognised. The following pages are intended rather as a brief guide to the subject than as a detailed survey. The latter must be sought in one of the many excellent works on the diseases of children. Here I shall consider only the broader principles, which grow into prominence as experience sorts observations in a clear perspective. My intention is to throw into relief from a clinical standpoint the principal distinctions between young and adult patients.

Though infirmity, as Malvolio says, decays the wise, it is in the knowledge of us all that the features of disease, reflected by the mirror of neurosis on to the placid surface of health, are the treasured possessions of many. The lives of these are empty of all but trivial incidents, and their very shallowness strands them in miserable contemplation of their own sensations. To them a natural function naturally performed is a subject for morbid apprehension. A minor malady becomes an event and a surgical operation marks an epoch. With children we have to deal with another class. The mere act of living is to them a supreme joy. Health and ill-health are terms outside their knowledge, and they have yet to learn the dubious pleasure of running after unhappiness. Herein lies the first peculiarity in the attitude of the young towards disease. As their days are innocent of care, so each leaves an added zest for the morrow. But if illness intrudes, their childish pleasures must be foregone. Their restless energy must be pent between the sheets, and, overfilling their cup, come the discomfort, malaise, and pain of the illness itself.

The important lesson to be drawn from this is that children do not make a business of their health. They do not, like many of their elders, suck the bitter-sweet of valetudinarianism for any morbid satisfaction it may yield. They dislike illness, and their natures are too genuine to suggest the assumption of its features for ulterior motives. If, therefore, a child shows a change, temporary or lasting, sudden or gradual, in his habits of life, it is right to look to his bodily health as the probable 
cause. If a child begins to lose interest in the society of his playmates or in his school-work, his health is surely slipping from him, and we cannot often explain the alteration in any other way. This characteristic antipathy to disease not only prevents children from simulating illness, but, when sickness has marked them down, keeps them from multiplying their symptoms. They are uninfluenced either by habits of morbid introspection, or by any desire to enlarge the gravity of their condition in order to make themselves the petted centre of their little circle. This last motive, which, with a craving for unearned sympathy, is a factor so often presented to the physician in his dealings with adult patients, is relatively uncommon among young children. Of all sick persons, perhaps none so wants sympathy as the sick child. To his apprehensions a mother's arms are narcotic, yet he seeks no more than his just meed, and his mental attitude can be relied on as an unassumed and unexaggerated index to his bodily state.

Although I have thus emphasised the genuineness of little children, sham and imaginary complaints are by no means strangers in their sickrooms. Schoolboys are notorious, though often transparent, professors of the art of malingering. Their ingenuity and resource will often baffle their parents or masters, and may even tax the acumen of their medical men. Simulation apart, true hysteria is not the exclusive heritage of adult women. Its occurrence in childhood is well recognised, and its possible existence must be borne in mind, even in the case of patients who have but the other day cut their milk-teeth. It has developed in the third year of life, but is rare until four or five years later, and even then is not common. One of the noticeable features of the disease in early life is that it affects boys almost as frequently as girls.

The great muscular activity of a child is responsible for some peculiarities in illness. An adult may have an insidious complaint creeping over him for a long time before his vigour shows any appreciable change. Even when attacked by one of the more acute diseases, he will often, by force of will, continue his daily round for a time. Not so with a child. One of the earliest signs of a slow-growing disease is a notice- 
able disinclination from the exercise which has hitherto been eagerly sought. At first the less energetic pastimes are preferred to the more exhausting. Later, quiet games supplant the active. Finally, he needs persuasion to pursue any interest that is not wholly sedentary, and only by urging will he join in play with others. Similarly in his acute maladies the first sign of ill-health is often a wish to lie down.

This slackening of a child's physical energy is usually associated with a loss of interest in his surroundings. The exertion demanded by the attentive attitude becomes too great. $\mathrm{He}$ grows fatigued in trying to observe the innumerable incidents that have previously been full of attraction to his enquiring mind. He avoids his companions. Their incessant movements, their shouts, even their quieter conversation tire him. He mopes at home; at school he is called a dullard. Now the mind of a healthy child should find novelty at every turn, and though this alertness of attention is sometimes discouraged as leading to incessant question asking, it is nevertheless an almost infallible sign of health. Any unnatural decline in its vigour is, therefore, often the first intimation that the child is beginning to ail.

In this connection it should be noted that a child who is in a state of depressed vitality is a readier prey to bacterial diseases. He also shows a peculiar liability to those involuntary muscular movements, which, under the name of "habit-spasms," are so common in school years. Some children, whenever their health falls below par, begin this twitching. The movements are usually of one or both eyes, of the shoulder, and, less commonly, of the muscles of some other part. Some children are prone to walk in their sleep at these times. In almost all cases, however, these troubles pass away as the general health improves.

Turning now to another peculiarity of children in disease, we find that the prominent place occupied by growth in the physiological processes of early life suggests a similar importance for it in the present connection. Regular and normal increase, both in stature and in weight, can only proceed under circumstances which make for health. In the absence of any of them, 
or in the presence of unhealthy conditions, progress may be retarded or even prematurely arrested. This interference, however, is often so slight, and the rate of growth in healthy children so variable, that the evidence it affords is not, as a rule, available until the adverse influences have been at work for some considerable time. Many brief illnesses leave no appreciable mark on the child's stature or weight. Therefore, as an early or even moderately early indication of ill-health, growth is not of the greatest importance. But the same reasons that lead to this conclusion also show that an interruption in growth, when once it is obvious enough to obtain recognition, is a serious warning of an abnormal state that must have continued over some considerable period.

The existence of the relation between growth and foodsupply has already been discussed in an earlier chapter, but we have now to realise that even children who are well fed may fail to make satisfactory progress. A part from tuberculosis and the wasting diseases of infancy-the latter of which are usually dependent on errors of diet-certain diseases are peculiarly active in retarding growth in volume or in height. The specific fevers, especially measles and chicken-pox, often produce this result. Another of the most frequent causes is acquired heartdisease. No one who has examined many children can fail to have observed that heart-disease presents a different clinical picture according as it attacks adults or adolescents. The point of distinction is that in the one case growth is complete and cannot be affected, while in the other it may be seriously hindered. In children, heart-disease, until it is leading the sufferer near to death, is not usually a disease of failing circulation-swollen ankles, congested lungs, pain, breathlessness, and the rest-but a disease of growth. It is essentially a wasting disease. Many of its victims are brought by their parents to the doctor solely on account of their wasting. Where the severity of the lesion is not enough to prevent the completion of adolescence, the fully-grown patient will sometimes be of a well-formed but diminutive build. Many young women who are of the "petite" type will be found to have had a constriction of the mitral valve from early youth. 
It is not without interest to observe that heart-disease in children seems to follow one of two types. In some cases the body as a whole appears to concede its rights for the benefit of the diseased organ. Growth is stunted, but the heart is able to maintain the circulation through the small body. In other cases, growth continues in spite of the additional work thereby cast on the already embarrassed heart. In these patients the circulation soon fails, and the adult form of the disease is produced.

Children then show the impress of ill-health in their rates of growth. Associated with this we often find variations in appetite. Whether it is that the poor appetite results in slower growth, or that a feebler demand for building material leads to a smaller supply, is difficult to decide. There can, however, be no doubt that the desire for food, though profoundly modified by disease, waxes and wanes wholly apart from any recognisable morbid factor. In adolescents, appetite must vary with growth as well as with the wants of existing tissues, and, since the rate of growth is variable, an indifferent appetite may mean nothing more than a lull in growth. If, however, aversion from food has been long continued, or is associated with any abnormal signs, it must be regarded with suspicion. A disinclination, or even an active dislike for food, not only accompanies many diseases, but may continue for months afterwards. So frequently is this the effect of measles and chicken-pox, that loss of appetite should always prompt an enquiry for the recent occurrence of one of these complaints.

Acute Illness.-Limiting our attention to the subjects of acute illness, we find many clinical features which mark off children from adults.

The symptoms of acute disease, which in adults are often restricted chiefly to the organ or region primarily involved, tend in children to affect the patient as a whole. Expressing this in another way, we may say that children develop general or constitutional symptoms more often and on less provocation than adults. This observation may be amplified by the statement that the younger the child the more striking is the contrast, and that in all of them localising symptoms are less 
obvious. The explanation of this difference is to be sought in a physiological direction. In the very young there is, as we have seen, less specialisation of nervous function. This imperfect development specially affects the mechanism of inhibitory control. Reflex actions are unrestrained and become quickly generalised; therefore constitutional symptoms are readily induced. These latter disturbances often arise in children as a result of conditions which are inert in this direction in adults. Consequently, nervous symptoms, which in the latter portend grave issues, may develop in early life without implying cause for any serious misgiving.

In conformity with these peculiarities, we find that most of the acute diseases of childhood frequently give rise at their onset to one or more epileptiform convulsions-an accompaniment practically unknown in patients of mature years. Though their severity may excite alarm in the minds of the inexperienced, they do not, as a rule, produce any but transient effects. Once the disease is fairly established, they usually subside. The specific fevers, diarrhœa and gastro-enteritis, which do not provoke convulsions in adults, will often lay hold of a child by means of this initial phenomenon. An attack of pneumonia not infrequently introduces itself by a series of epileptiform seizures. In conditions such as meningitis, cerebral tumour, or uræmia, which in adults are usually associated with convulsions, children, as would be anticipated, reveal an exaggerated tendency to the fits, which may follow each other without lull or intermission till the patient is exhausted.

The clinical complex that goes by the name of a "cerebral state" and comprises blurring of the mental faculties, drowsiness, delirium, and coma, though not rare at any age, is induced most readily in the young. These signs are therefore of less ominous import in early life. Acute pneumonia, whether beginning with convulsions or not, may set up this cerebral state quite early in its course and before any localising signs have developed in the lungs. In adults, on the other hand, such a condition does not as a rule arise until the disease is passing towards a fatal issue. 
Another and even more important feature of childhood is the rapidity and frequency with which its acute diseases alter their course. During their progress the pulse and temperature are subject to profound disturbance and the general condition of a case may undergo quick changes for the better or worse. These vacillations are often ascribed to some slight indiscretion in diet, to exposure to cold or to some other seemingly unimportant cause, but not infrequently their origin cannot be traced. Without any warning, a child whose condition seems free from anxiety may quickly pass into a precarious state. The converse, however, holds equally true. The importance of these points as regards caution in prognosis and unremitting perseverance of treatment is obvious.

When the acuteness of the attack begins to subside, the symptoms often pass away rapidly and the child may soon regain his liveliness and bright intelligence. Sometimes, indeed, the mists seem to roll back with almost startling swiftness. Convalescence often progresses at a quick pace. Just as a decline of vigour first gives warning of an oncoming disease, so its revival is one of the earliest signs of a return of health. The speed with which energy both of muscle and of mind is regained forms one of the most gratifying features of sick childhood. This recovery, however, has to be regarded with caution, because it often outstrips the repair of the organ or organs affected. These may still continue in a pathological state, and not uncommonly a secondary illness is set up some time after the general appearance of the child has suggested that all trouble is past. The frequency with which sequelæ chequer the convalescent period is characteristic of the age. It warns us to show no undue haste in relaxing the restrictions of the sick-room. Finally, the course run by a sequela or by a complication is often as irregular as the original attack.

If we were to sum up the attitude of children to acute disease, we should say that their equilibrium is shifting. They are easily overturned, but as easily set up again. Their centre of gravity is high in health, and they are soon knocked down. In disease it lies low, and but little is needed to put them on 
their feet again. The extent to which they bend before a storm is no exact measure of the furiousness of the assault. Yet the very malleability of constitution that enables them to withstand the gravest attacks must also help to swell the roll of those who succumb to mild diseases. The most serious aspect of this matter is seen in the frequency with which apparently trivial complaints end fatally. Not only does death in early life come more suddenly and with less warning than in later years, but sufficient cause for it is most often lacking in autopsies on the young. Of the recognised causes of sudden death in children the factor common to most will be found to be an interference with the freedom of breathing. This holds for atelectasis, pressure of enlarged glands or of a retro-pharyngeal abscess on the trachea, asphyxiation from food in the airpassages, from an enlarged thymus, and from overlaying. These are the causes that most frequently account for sudden death in infants. In other words, the situation immediately responsible for death is reached only shortly before the end, and but little time is left to produce any marked changes in the organs. 


\section{CHAPTER XIII}

\section{ON EXAMINING INFANTS AND CHILDREN}

The Child's Fear of the Doctor ; Crying assists the Examination in some respects; Position of the Patient; Medical History; Value of the Mother's Observations; General Inspection of the Patient; Painful Examinations; Risks in handling the Child, in impeding his movements, in startling him; Auscultation; Procedure; Percussion ; Abdominal Examination; Examination of the Nervous System; Rectal Examination; Examination of the Urine ; Examination of the Blood; Temperature Records; Examination of the Pulse; Examination of the Sputum; Examination of the Vomit and the Fæces; Examination of the Tonsils.

In the present chapter I shall offer some practical suggestions for the successful physical examination of infants and children, but I shall attempt no more than the loose weaving together of those methods which I have put to the test of experience.

Even the youngest child has likes and dislikes. Some he shares with all other infants of his own age. Some are peculiar to himself. Like his elders, he resents infringements of his weaknesses. As a general rule the younger a baby the more he will resemble other babies and the fewer will be his idiosyncrasies, so that the methods which succeed with one infant will succeed with another. Later, especially after a child has learnt to talk and the development of his imagination has led to a growth of the instinct of fear, he must be approached in a particular way to win his own particular confidence. It is important to recognise this twofold constitution of a child-the specific and the individual-because it provides the means of securing his confidence. 
It is hardly necessary to say that before embarking on a physical examination, a friendly relationship must be established with the child. It is somewhat difficult to frame directions for accomplishing this end. Many ways, doubtless, are equally efficacious. Some, however, which are successful in the hands of one, may be useless when employed by another. Others, again, may appeal to one child and yet may alarm his fellow. Time spent in dispelling the nursery prejudice against doctors is not wasted, for it will be saved later when the patient contentedly allows a detailed investigation and provides facts that could not possibly be obtained from a nervous child. Infants of a few months necessarily exhibit no special timidity towards the doctor, and with them we have no initial bias to overcome. But though they draw no distinction between their medical and their lay visitors, they group both in the single category of strangers, and these are likely to be received with alarm, perhaps with crying. It is usually desirable to avoid this occurrence, though in certain cases crying may be useful by facilitating the auscultation of the lungs. Thus, young infants are apt to allow very long pauses between their respirations, and when an inspiration is at last taken, it may be too shallow to be of service. This difficulty does not occur if the patient is crying. The inspirations are then of considerable depth, and, though their rhythm may be slow (owing to the habit which babies have, when annoyed, of holding their breath), any alteration of the vesicular murmur or the presence of adventitious sounds is readily heard. A lustily crying infant is the only one on whom the character or degree of vocal resonance can be tested, for it is hardly possible to induce him to phonate at the precise moment when the presence or absence of bronchophony is to be ascertained.

The first care, then, must be to make the infant feel "at home," and this comfortable attitude cannot be found outside the mother's or nurse's arms. It is a mistake to direct a mother to place her baby on a couch in order, for example, to palpate his abdomen. The almost invariable result will be that the child loudly resents the change and his abdominal walls at once become too rigid for the examination. No infant is likely 
to be as contented out of his mother's arms as in them, and it is only wise to complete as much of the examination as possible with this assistance. There are other details necessary to observe at this stage. If the patient is awake he will probably turn to study the doctor. The latter will be wise to take no notice at first, but to give the child ample time and opportunity to complete his investigation. He should not sit too far away for the child to watch him easily, but he must be careful not to approach too near, as the sudden looming-up of an unknown face is quite sufficient to cause alarm. When he has placed himself at appropriate distance, it is advisable to begin at once to talk either to the patient or to the mother. This is of some importance. The child after recognising him as one of the same species as his parents, not unnaturally expects him to emit similar sounds, and he may, therefore, become apprehensive if disappointed in this respect. While the doctor is quietly interrogating the mother, the child will have ample opportunity to understand that there is no need for anxiety, and when the history has been obtained, notice can then be turned to the patient.

In dealing with an older child, the object should be to draw him into conversation, and this may conveniently be done by questioning. A child that is nervous towards strangers is not likely to be conversational, and therefore, as soon as he volunteers a remark over and above his reply, his shyness may safely be looked on as evaporating. One often hears an adult attempting to break the ice by a series of questions to which replies, however brief or even monosyllabic, will be necessary. Obviously the method is a good one, otherwise it would not be so commonly employed, but it is not the best. One of the first effects of shyness is to make children lose their tongues. They have not a word to say and do not want to be asked to find one, and if their replies are deliberately awaited they may feel that they are being unfairly pressed. A better plan is to talk to the child, without expecting replies, until he shows signs of emerging from his shell, when he can be drawn out by a welldirected question or two. Sometimes the shyest children are just a little frightened by the mere fact that remarks are 
addressed to them directly. Experience soon teaches the interpretation of the fleeting expression in a child's face or attitude, and, if any apprehensiveness is shown, it is best to turn the observations to the mother, while we continue the same topic in such a way as to tempt the child to join in.

Before attending to the patient we should set about obtaining the medical history. This is best sought from the mother to whom the child, if old enough, has probably described his subjective symptoms. Information on such points is often extracted with difficulty from the patient-in fact free denials in this connection must be expected, for many children leave their pain and all knowledge of it outside the consulting-room. With regard to the value of the history as given by the mother, it must be remembered that she is in a much better position than the doctor to watch the course and fluctuations of her child's health, but that she is naturally apt to be biassed in their interpretation. It is often surprising how even a most careful mother will overlook a prominent symptom until it is enquired for. Her attempts to locate her child's pain may be wide of the mark, and, for example, a definite history of severe headache may be founded on nothing more than the childish habit of lying or sleeping with one hand to the head-an attitude that may mean something or nothing. Or again, pain may be described as abdominal because the thighs are flexed over the body-a position that many babies habitually assume for some weeks after birth. In all cases it is well for the doctor to depend rather on his own observation and examination than on the contrary evidence of others.

Suppose the history obtained, the child friendly and the road clear for the actual physical examination; what is the best way to undertake it? The first essential, after the position of the patient has been seen to, is to have all the clothing removed. A warm wrap may be thrown round the child if the day is cold, but by preference the chairs should be drawn to the fire and the child left completely uncovered. This will afford an uninterrupted view of the whole body and enable the movements of the abdomen, chest, and limbs-items of great 
importance-to be studied in detail. As he reposes comfortably on his mother's knees run your eye over him from head to foot, front and back. Note his size and compare this with his age. Observe his general nutrition and the firmness or flabbiness of his flesh. Study his facial expression and his complexion. Is he dull, bright, listless, apathetic? Does he cry, and if so, is it the loud cry of temper or of hunger, the intermittent, sudden outbursts suggestive of pain, the longdrawn wail of continued suffering, the sharp penetrating cry of brain trouble, or the mournful whine of utter exhaustion? Watch his respiration, noting whether it is easy or laboured; whether there is sucking-in about the chest; whether the alæ nasi work. The rhythm of breathing, is it regular and quiet, or jerky with a pause at the end of inspiration succeeded by a grunting expiration? The presence of a cough is important. There is the croup of laryngitis, the oft-repeated cough of pneumonia-little more than a clearing of the throat-the violent paroxysm of whooping-cough.

Notice the contour of the head. The well-known features of the rachitic head have to be distinguished from the globular bulge of the hydrocephalic or from the syphilitic marked by its osteophytic ridges like a hot-cross bun. Ascertain the condition of the anterior fontanelle, whether it projects, sinks in, or pulsates. Next, carry your eye down the neck to the chest where many points must be observed. The two sides should be symmetrical and evenly moulded. Note whether the wall sinks in on either side of the sternum, whether the sides of the thorax, especially at their lower parts, are no longer convex but concave, showing Harrison's sulcus. Beading of the ribs, pigeon-breast, sinking in of the intercostal spaces, præcordial bulging, forcible or diffuse cardiac impulse are all important aids to diagnosis. The abdomen is normally large in a child, and a tumidity approaching pot-belly may imply nothing amiss. Is there any local swelling? Is the epigastrium unduly full? Are the veins dilated? Glance at the navel, the inguinal rings, the prepuce and the buttocks. Finally the limbs require notice. Observe whether each limb is being moved freely or whether one is kept motionless or used unwillingly. Ascertain the 
condition of the epiphyses and the presence or absence of clubbing of the fingers.

All these items may be studied before the child is so much as touched. They have taken some minutes to describe, but with practice they can be observed almost as soon as the baby is stripped.

When a mother sees that the examination is about to begin, it is not unusual for her to alter her infant from a sitting to a lying position with the intention of facilitating the examination. This sudden change from the vertical to the horizontal is likely to provoke struggles and crying by way of protest. It is well to forestall this unnecessary interruption by telling each mother as a routine practice, and before she has had an opportunity of acting otherwise, that the child is to be placed upright, supported or not by her hand, according to his age. This precaution does not apply, of course, to children who are seriously ill and must be kept in a horizontal position.

For his own welfare, the medical man should be careful not to allow the baby, if a boy, to sit directly facing him.

It is hardly necessary to say that nothing should be done that will hurt a child. If an area of skin, a joint or a bone is found to be tender, its examination should not be pressed. Only exceptionally is it required to investigate in detail a region that is painful to the touch, and even then this step should be postponed until the last.

In making an examination three precautions must be observed. Don't handle the child more than is absolutely necessary. Don't impede the movements of its limbs or head. Don't startle it by unexpected actions.

The importance of these points will be best recognised by illustrations. An inexperienced student may sometimes be seen to begin his examination by palpating the child all over, first feeling the fontanelles and cranial sutures, then drawing the lips up and down to expose the teeth, next feeling the bones of the arms, working the joints open and shut like so many hinges, finally passing to the legs, observing, en route, any possible beading of the ribs. Needless to say the child is screaming lustily long before the voyage of discovery is com- 
pleted. A small infant, resenting the sudden application of the cold chest-piece of a stethoscope to his skin, attempts to push away the offending instrument. His arm is promptly seized and imprisoned. The other hand coming to the rescue is caught up and held between a spare couple of fingers, and in a few seconds the child is violently struggling to liberate himself and soon wriggles off his mother's lap. Babies are quick to resent treatment of this kind, and it inevitably leads to trouble.

With regard to startling the child by unexpected actions, I do not mean that he should be warned before each step is taken. On the contrary, an attempt should be made to fix the child's interest on anything rather than on the examination, but at the same time care must be taken to avoid provoking a sensation in any way unpleasant or likely to claim the child's principal attention. For example, when auscultation is commenced at one base, the sudden application of the stethoscope will reflexly cause the child to start and therefore to be alarmed. If now the chest-piece is placed over the opposite base, there will again be the reflex jump, and the child will possibly cry. Similarly, the sudden contact of a cold hand with the abdomen will mean unnecessary risk of disturbance. It is a golden rule in children's practice to avoid setting up cutaneous sensations as much as possible. Touch only when you must touch, and then firmly rather than lightly in order to let the child recognise the feel of the hand. In palpation, the hand should be passed slowly, not quickly, over the skin.

Humour the child, and never be in a hurry with him. Should he take a fancy to your stethoscope, let it be a toy for the minute. A little practice will soon enable you to differentiate between the vibrations thus set up and the pulmonary or cardiac sounds. In any event the novelty of the toy will soon pass. It is sometimes a good plan to introduce the several instruments as so many toys which the child is to be allowed to touch or even to hold so that he may lose all apprehensions concerning them. Quite small babies are too young to be interested in toys, but bright articles appeal to them. A pair of bright sleeve-links or of gold-rimmed glasses may be irresistible.

Now to the actual examination. Is there a set routine of 
investigation in children as in adults? No. It is not often advisable to endeavour to examine children in a methodical way because the attempt will fail as often as succeed. Experience will soon teach you that the most satisfactory course is to be keenly on the alert for each opportunity the child offers, and to seize it before it has passed. The plan has one drawback, namely, that some important point may be overlooked; but ordinary care should avoid this difficulty, especially if, when nearing the conclusion of the examination, you run through the various items in your mind. For myself, I make it a general rule to examine first those organs which will probably be found healthy, as thereby pain is not likely to be inadvertently caused. For example, in the case of a child with scurvy rickets, the limbs should not be examined at the outset, nor the abdomen of one suspected of tuberculous peritonitis.

Auscultation.-On most occasions auscultation may be employed at an early stage, but the first use of the stethoscope should be made with caution. If the child objects to the instrument altogether, another point in the examination may be taken, and auscultation reserved till later. Auscultation is, however, of considerable importance, and its thorough completion must not be omitted. In the first place, it is safer to listen to the back of the chest before the front, as the child is less likely to take alarm if the stethoscope is out of sight. Care is needed until the patient has grown accustomed to the feel of the chest-piece, and a good deal depends on the initial move. A cold chest-piece must not of course be used-it may be warmed for a few moments in the hollow of the hand-but the sensation even of the warm metal or vulcanite is quite commonly resented. Against this the following ruse is often successful.

The mother is asked to turn her child round so that its back may be first examined. This she will do by placing one hand on each side of the thorax under the armpits, lifting the child above her lap, and rotating it through $180^{\circ}$. As she takes her near hand away one of the doctor's hands is slipped in its place, and the infant does not notice the change. Next the hand is slowly spread, and the doctor, 
having gone thus far without raising suspicion, places the warm chest-piece in the cleft between his fingers. Finally, the hand is withdrawn, leaving the instrument against the skin. This manœuvre rarely fails, but if the child begins to show signs of restlessness when the touch of the stethoscope is felt, the thumb, which has not been allowed to come in contact with the skin, is stretched as far away from the fingers as possible, and its tip rubbed gently against the lower part of the back. The child's attention is at once successfully attracted from the stethoscope to the friction of his skin, the whole hand is withdrawn, and the stethoscope remains in undisputed possession.

When the chest-piece is to be moved to another area it should be transferred cautiously. Its unexpected pressure on a remote part of the chest-wall is apt to cause the same trouble as before, and therefore it is best to move it only a short distance from its first position, or even to include part of the same area again. Then its range is gradually enlarged before passing to the opposite side. In many small children in whom the lower dorsal reflex is brisk, the stimulus of the stethoscope applied to the base of one lung often causes the back to straighten suddenly, and this may be resented. If, however, the progress is made from above downwards, this little difficulty is not likely to occur.

When the examination of the back has been completed, attention must be given to the front. Having grown accustomed to the feel of the stethoscope, the child does not as a rule object when it appears before his face ; but, nevertheless, it is prudent to approach the front carefully. The patient need not be moved round to face the examiner, but the stethoscope should creep round as it were from one axilla. On gaining the anterior surface of the chest, we should first examine the lower part-for example, the cardiac impulse, rather than the apex of a lung-as we run less risk of alarming the child, than by beginning high up near his face.

Percussion.-After auscultation it is generally a simple matter to proceed to percussion. This method is to be applied gently. The vigorous strokes that may be necessary when dealing with an adult are rarely, if ever, desirable in the case 
of a small child, for the vibrations set up are warranted not only to alarm the pationt but to give misleading information. With one finger laid fairly firmly on the chest-wall, percussion is to be lightly made with the opposite hand. It is the most delicate percussion that brings out distinctions in resonance.

Abdominal Examination.-Abdominal examination is not as a rule difficult. The muscles being weak, the walls are soft and supple, so that even the posterior parts of the abdominal cavity can be explored with ease. On the other hand, gastric, enteric, and colic troubles are so common in sick children that some degree of abdominal tenderness is frequent. In these cases the local examination should be reduced to a minimum. To palpate the abdomen the infant should be sitting on his mother's lap looking towards the examiner's right. The left hand should be placed behind the small of his back as a support and counter-pressure to the palpating hand. The warm right hand can then be laid on the front of the abdomen, but without making pressure, and allowed to remain until the child's expression shows that it is safe to proceed. It is a mistake to be in a hurry to begin palpating, because the slightest objection on the child's part means rigidly held abdominal walls and failure. When the road is clear the pressure of the fingers may be slightly increased, and then relaxed, and in this way a gradual passage made to the complete examination. The same precautions that require to be observed in palpation of an adult abdomen are as necessary here-the whole hand must be laid flat on the abdornen, and the use of the finger-tips must be avoided.

Nervous System.-The detailed examination of the nervous system in babies is not easy. In adults we rely to a great extent on the active co-operation of our patients, but this assistance is, of course, not forthcoming from small children. Such points, however, as inequality of the pupils, nystagmus, strabismus, facial paralysis, muscular rigidity, head-retraction, and limb-palsies are ascertained without difficulty. With the last-named condition care is necessary to distinguish a real paralysis from what is often called a pseudo-paralysis, that is, an immobility of a limb due to epiphysial or other bone trouble. 
Paræsthesia, paresis, and tendon-reflexes-the last of which must be investigated with the help of a percussor-are often difficult to detect, while incontinence of urine and fæces is of little importance in babies. Slight motor disturbances in a somewhat older child can be best discovered by persuading the patient to walk, or by dangling a bright or attractive object, and observing whether one arm is used to seize it in preference to the other. Ophthalmoscopy calls for the exercise of more patience than any other examination. The light reflected from the mirror will probably either annoy the child or cause him to close his eyes in sleep. In each case the aim of the examination is frustated. If neither of these results follows, and the patient continues to look about, the examination may require much time, and at best only transient glimpses of the blind spot are likely to be obtained. It is possible to examine the fundus of a sleeping child, but the eyelid must be raised very gently, and the position of the head left undisturbed. The direct method of examination is generally preferable to the indirect.

Rectal Examination is a specially valuable procedure in the young, inasmuch as the length of the forefinger will carry the investigation high into the abdomen. It is not as a rule objected to, provided it is carried out with all gentleness, and the finger passed very slowly indeed into the bowel. As regards his attitude the child may be sitting up, but preferably should be lying down on his back or on his side.

Urine.-With small children specimens of urine are difficult to obtain, but, in cases in which it is desired to ascertain its composition, one of the following methods must be employed. A soft, small-meshed sponge or pad of absorbent wool must be placed against the urinary orifice and retained in position by the napkin tied in the usual way. The sponge must be removed as soon as possible after the urine has been voided, and for this purpose it should be examined at frequent intervals. With boys it is often possible to fasten a test-tube by a strip of adhesive strapping to the pubes, and so to arrange it that its mouth surrounds the penis. In all important cases, however, the urine must be obtained by catheterisation. 
Blood.-A drop of blood is best obtained from the lobe of the ear. Not only is this part less sensitive than the finger, but the patient does not see the result of the puncture. If the finger is used, however, it is often possible to persuade an older child to watch with interest the gradual appearance of the drop, and he is thus made to forget his fear.

Temperature.-For accurate records this must be taken in the bowel, and this method presents less objection applied to children than to adults. The thermometer should be vaselined and passed about one inch into the rectum. If an approximate reading is sufficient the axilla or, better, the groin is used. It is always advisable that the morning record should be made before the patient is washed, and the evening record before he has been undressed for the night.

Pulse-Little information can be obtained by examining the radial pulse in infants, and the state of the circulation is best ascertained at the anterior fontanelle. At a somewhat later age when this opening has ossified, as much may be learnt from the temporal as from the radial artery. This alternative is worth remembering when a child refuses to give his hand or persists in moving his arm about.

Sputum.-The difficulty of obtaining sputum by natural means is usually very great, as children swallow their expectoration. Sometimes, after an attack of coughing, it may be possible to persuade them to spit out the phlegm, or, failing this, the fauces may be at once wiped out with a cotton-wool plug. Of other methods that have been recommended we may mention the following:-The epiglottis is lightly touched by a piece of dry gauze on the end of an artery-forceps; the irritation causes a sudden cough, and any sputum expelled from the larynx will be driven against the gauze, to which it will adhere. Or the stomach of a patient who has passed the night without food is washed out, and the sputum swallowed during the last few hours is thus recovered.

Vomit and Faces.-The customary methods of examination are as applicable to children as to adults. It is of very great importance that the doctor should inspect these dejecta for himself, as the presence of undigested food, blood, or mucus 
may give a clue to the diagnosis or suggest how treatment should be amended.

Tonsils, etc.-In conclusion there is one investigation that should always be deferred until the end of the examination. No child, however plucky, or whatever his age, enjoys having his throat examined. The mere attempt to inspect the mouth and fauces very often leads to trouble. Whatever the particular condition may be that calls for this examination-enlarged tonsils, adenoids, Koplik's spots, dental caries, stomatitis, cleft palate, or a furred tongue-it should be left undecided until the last. As a rule it is inadvisable to attempt to hide the tonguedepressor, and the child should be given full opportunity to appreciate its harmlessness. This examination, like others, should be made gradually. The spatula should at first be inserted only a short distance into the mouth and only later slipped back to the fauces. At the same time the child is encouraged to take deep breaths. If trouble in keeping the mouth open is anticipated, the depressor should be so manœuvred as to catch the patient's under-lip between the instrument and his lower teeth. For his own comfort he is compelled to keep his mouth wide open. 


\section{CHAPTER XIV}

\section{THE DIAGNOSIS OF DISEASE IN CHILDREN}

General Considerations: Absence of Subjective Symptoms; Sex ; Age; Season of Year; Prevalent Epidemics; Relative Frequency of Diseases. Diagnostic Value of Clinical. Signs and Symptoms : General Appearance of the Patient ; Importance of Minute Observation ; Diagnostic Value of Pain; Condition of the Anterior Fontanelle ; Weight and Growth; Deformities of Bones; Abdominal Examination ; The Fauces; Temperature; Pulse; Respiration; Expectoration ; Vomiting ; Constipation; Diarrhœa ; Epistaxis ; Rigors. Diagnosis OF Diseases of THE HEART AND of THE Lungs: The Heart; The Lungs; Peculiarities of the Healthy Vesicular Murmur ; Auscultatory Signs of Pneumonia ; Unequal Expansion of the Lungs ; Transmission of Signs from one Lung to the other; Auscultatory Signs of Pleuritic Effusion.

SOME of the difficulties that commonly present themselves in the diagnosis of children's ailments have already been mentioned in a previous chapter, where it was pointed out that in early life the subjects of disease rather than the diseases themselves are peculiar. Inasmuch as accuracy of diagnosis is of first importance from a medical point of view, no fact or observation that enlarges our knowledge of children is without value in the investigation of the condition of a sick child. To make an accurate diagnosis we must know the features of our young patients in health as well as in disease; and, therefore, few of the facts with which we have dealt in the preceding pages will be without their bearing on this question of diagnosis. The reader can be left to apply them to each case according to his experience. It will be remembered, however, that attention was specially drawn to the comparatively slight influence of 
mind on disease in young subjects, and this peculiarity must receive full recognition.

The diagnostic field is appreciably narrowed by the fact that its boundaries exclude the varying aspects of hysterical and neurotic affections. However, the resulting simplicity is more than counterbalanced by a difficulty which at first misleads all students of children's diseases. Young patients are unable fully to express their subjective symptoms, and timidity often leads them to deny the existence of an important phenomenon. From infants, of course, no help can be expected, and the doctor must rely on his own powers of observation and on his past experience. The absence of the patient's own story leaves a gap which is specially felt, because we have all been taught to attach great importance to this history in the case of adults. After a time, however, the want seems less inconvenient, and later still it may be appreciated as an advantage, inasmuch as the diagnosis is left to the medical man's own trained skill, unbiassed by misleading or inaccurate statements from the patient.

Certain of the personal attributes of a patient as well as some general factors influencing disease have a relatively different value in children and in adults.

Sex.-The question of sex which is of cardinal importance after puberty has but little bearing on diagnosis in the very young. Sexual characteristics, either physical or mental, are still imperfectly developed, and disturbances caused by them are improbable until a later day. The conditions of life in the two sexes have not yet been specialised, and dissimilarity of environment has not yet exerted its influences. Any one disease, therefore, is as likely to occur in boys as in girls. The few exceptions to this include none but the less common conditions - such as pseudo-hypertrophic paralysis - and their practical value therefore is small.

Age.-Though sex is a subordinate factor the precise age of a patient is often the foundation of a diagnosis. The question has already been approached in connection with the incidence of disease (p. 265), and the importance of the point may be emphasised by a single illustration. Heart-disease is common 
in children, and organic murmurs may be heard at every age. They arise from deformity of the heart or from endocarditis acquired as the result of rheumatic or scarlet fever. The former variety is usually incompatible with prolonged life, but the latter is often of much less gravity. The conditions must therefore be carefully distinguished. Apart from every other means the age of the patient will often suffice to settle the point without reasonable doubt. Thus, the maldevelopment of the heart is congenital and the bruits are heard from birth onwards, while acquired heart-disease is very rare indeed under three years, and is rare until four or five years of age.

Season.-The influence of time must be allowed not only in respect of the age of the patient, but also in regard to the season of the year in which an illness occurs. A considerable number of both infantile and juvenile diseases show a distinct seasonal periodicity, and while frequent or even epidemic at one time of the year, are uncommon or rare at another. The acute gastro-enteritis of infants is one of the best instances of this. It is rampant in the summer months, but disappears in winter. Typhoid fever and diphtheria are most prevalent in the autumn months, while whooping-cough, pneumonia, and cerebro-spinal meningitis are more frequent in the winter and spring. Measles, though occurring at all seasons, is commonest in the colder months, German measles in the spring and early summer, while rheumatic fever is stated to reach its maximum in London in September and October. The interesting condition known as spasmus nutans begins, in the majority of cases, during the four months November to February.

Prevalent Epidemic.-In every case of acute illness due consideration should be paid to the prevalence of any epidemic in the neighbourhood, or in any locality from which the child has recently been removed. By bearing this warning in mind the practitioner will be put on his guard, and with only the earliest symptoms to guide him, he will often be able to recognise the nature of a disease before it has assumed its characteristic features.

Relative Frequency of Diseases.-No less important, and in most cases more important than a knowledge of prevalent 
epidemics is the knowledge of the relative frequency of the commoner diseases of childhood. The number of these is not great, and, though the order of their importance is likely to vary from place to place, the same list will apply to most temperate climates. Urban and rural life exercise different pathological influences, and therefore it is not possible to arrange the items in an order that will be universally applicable. The following list of nine of the commonest acute illnesses is arranged approximately according to the frequency with which they are met in hospital practice :-

I. Acute Digestive Disturbances.

Acute Gastro-enteritis.

Acute Entero-colitis.

2. Acute Pulmonary Disturbances.

Acute Bronchitis.

Acute Broncho-pneumonia.

Acute Lobar Pneumonia.

3. Acute Tonsillitis.

4. Acute Specific Fevers.

5. Rheumatic Fever.

6. Tuberculosis.

7. Meningitis.

8. Acute Otitis.

9. Acute Nephritis.

This list applies both to infants and young children, and would require to be modified to suit one group alone. Thus the commonest acute affections of patients under one year do not include tonsillitis, rheumatic fever, or acute nephritis, while in children of school age, acute digestive and pulmonary disturbances would not occupy so prominent a position.

A corresponding list of the common chronic conditions (excluding dental caries, skin diseases, and defective sight) would comprise the following:-

I. Chronic Digestive Disturbances.

Marasmus from bad feeding.

Diarrhœea.

Vomiting.

2. Rickets. 
3. Enlarged Tonsils and Adenoids. Otorrhœea.

4. Heart-disease (rheumatic especially).

5. Chorea.

6. Tubercle.

7. Congenital Syphilis.

Here, again, the list must be re-arranged according to the age under consideration. Enlarged tonsils and adenoids, acquired heart-disease, and chorea are rare in the earliest years of life, but chronic digestive troubles, rickets, and congenital syphilis attain their greatest clinical importance during this period.

Thus far we have given attention only to those general considerations that affect children as a class, and we must now turn to the special clinical features arising in individual cases in order to learn their diagnostic interpretation and value.

General Appearance.-Too much importance cannot be attached to the general aspect of a sick child, but we must at once beware of attributing to the complex of symptoms that go to make up this aspect the same implication as we should in the case of an adult. Remembering the ease with which constitutional symptoms develop in early life from surprisingly trivial causes, we shall be wise if we look deeply into a case before deciding on its gravity. Especially desirable is this when we observe how readily cerebral symptoms appear without cerebral disease, and how coma, suggestive of ominous brain-trouble, may result from much less serious conditions. Such cases can be diagnosed only by the most careful examination, and it should never be forgotten that if symptoms such as delirium, drowsiness, or coma are the outcome of brain-trouble, other more definite indications of interference with the cerebral functions will probably be found. Apart from optic neuritis, we should patiently seek any muscular spasm or weakness, disturbed rhythm of heart or breathing. Loss of control over the bladder or rectum would, in older children, have a serious meaning.

Though the broad aspect of a case is highly suggestive, it would be unwise for an observer to allow it too much influence over his diagnosis. The general appearance is the impression 
obtained in a glance more or less subconsciously. Thus a mere look will suffice to identify each of three cases, of which one is heart-disease, another pneumonia, and the third meningitis. The heart-case half sits with his arms flung over his head, breathing with some distress, yet following the incidents of the ward with interest. The lung-case, propped up in bed, restless and anxious, thrusting the bed-clothes far away, is so intent on getting his breath as to resent interference of any sort, and even drinks his feeds only by momentary dashes at the cup. The brain-case lies curled up on one side, staring vacantly with filmy eyes, his head thrust deeply into the pillow or rolling from side to side. Impressions such as these are always serviceable, and should be sought in every case examined, whether child or adult, but we should not rest content with the subconscious impression alone. We should analyse it in order to determine its sensory components. The remark is often heard that a case "looks like" one of, say, typhoid fever or pneumonia, but if reasons for the comment are asked, they may be few in number and omit some important details. Yet if an observer, whenever a case has suggested itself as an example of this or that disease, were at once to set himself to ascertain why the idea has arisen in his mind, he would quickly sharpen his power of diagnosis by the necessity of recalling his past experience of similar cases.

The information that an onlooker can gather merely by watching his patient is often of the very greatest assistance, but in order that he may derive the fullest benefit from this inspection, he must be intimately acquainted with "the natural hue of health" as mirrored in each age of childhood. The ways of a babe differ at three and at nine months, and a child of five years and another of seven present many differences. Only by a knowledge of these dissimilarities will it be possible to judge of those minutia which are so suggestive to the trained eye. Mothers are keenly alive to changes in their own children, but they lack the skilled discrimination which alone can notice symptomatic details; and the doctor must be prepared to make his diagnosis without the voluntary assistance of the parent or the patient. Attention 
must be given not only to such noticeable characteristics as restlessness, listlessness, and taciturnity, but to every passing action of the child. His attitude and changes of attitude; the movements of his head, eyes, or limbs; the fleeting expressions of his face, his breathing, and a score of other points, are often full of suggestiveness, and just as they may betray an important symptom, so they may prevent a hasty diagnosis. Even in their sleep sick children are unlike their healthy companions. They moan or cry, throw off the bed-clothes, toss from this side to that, or fling their arms about. They may be difficult to rouse, or they may wake every few minutes. Sometimes they start up in a flood of tears.

A few illustrations taken from practice will serve to impress the importance of observing little details.

A child that is alarmed at the first approach of a doctor is not as a rule dangerously ill. If he were he would probably allow himself to be examined without offering resistance.

A child with suspected meningeal trouble who turns his head promptly and accurately to locate a noise in his neighbourhood is improbably the subject of meningitis. With this disease there would probably be no more than an irritable movement of the limbs.

A child with apparent head-retraction who is observed voluntarily to bend his head forward, is improbably the subject of cerebral disease; while another, with "cerebral" symptoms but a slack fontanelle, is not likely to have meningitis.

If an infant suspected of lung trouble shows the long pauses between his respirations so characteristic of healthy babies, he is improbably the subject of pneumonia. The same criticism would hold of the child who took more than a passing interest in its surroundings, for in pneumonia the attention is usually concentrated on the laboured breathing, which prevents any but a momentary notice of external affairs.

An infant who has been passing blood and mucus from the rectum for some hours, but sits up in his cot to see what is going on, is improbably an example of intussusception.

In every case of suspected grave disease the question is worth asking: "Is this child as ill in himself as he should be 
with this disease?" The precaution may not be infallible, but it will often prevent an erroneous diagnosis founded on a physical examination alone. It may sound trite to say that when a child looks ill to the experienced eye he is ill, but the platitude is worth keeping in mind when we see how frequently we may be misled by the features of adult patients. This is especially the case with adults who have been seized with alarming yet not dangerous symptoms. They look very ill, but really have not much amiss. Children are not, as a rule, apprehensive of their condition, and the newness of life prevents them understanding the meaning of death, but adults will suffer more from their apprehensions than from their illness.

Pain.-Among the many symptoms of disease none is of such frequent occurrence as pain, and none can rival it in diagnostic value. Its importance reaches a maximum in mature years, and at the outset of life it affords us comparatively little help. At the age when children have no power of verbal expression, it is not always easy to recognise its presence, character, or intensity, still less to localise it or trace its distribution. This comes not only from the infant's inability to speak, but also from his own ignorance of the site of his pain. His power of localising stimuli of every kind is still imperfectly developed. Consequently the two indications on which we must chiefly rely are crying and restlessness. Often a careful examination will throw light on the situation of the pain, as, for example, when pressure on the abdomen or passive movement of a limb provokes crying. The pain of hunger, however, is one of the most frequent causes of screaming, and the possibility of the severe pain of middle-ear disease must never be overlooked. Perhaps the leading characteristic of the cry of pain is its intermittence as each pang begins and ends.

The question is often asked whether infants feel pain acutely, and this has its bearing on diagnosis. If the outward expression of suffering were an index to its intensity, it would seem that pain does not play so important a part in children as in adults. An infant's power of expressing feeling of any kind is rudimentary, and he never shows a facial contortion characteristic of pain. Even in older children it is unusual to observe an 
emotional state comparable to the intense anguish that is seen in adults suffering from, say, renal colic. A more frequent result in early life is fear or even terror, and these psychical states return, often with increased vehemence, with every fresh attack. Even chronic painful conditions, such as tuberculous peritonitis, which might be expected to effect a change in the face of the patient, do not often, in earlier years, lead to an expression suggestive of the adult mask which results from prolonged suffering.

Pain that is imaginary, or at any rate exaggerated by mental process, is rare in childhood. On the other hand, referred pain-that is to say, pain referred to one healthy region of the body as a result of disease in another-is by no means unknown among those old enough to describe their own feelings. This possible interpretation of the symptom should not be forgotten. Its occurrence is only what would be expected, since referred pain results from anatomical conditions which exist at all ages. The pain of pleuropneumonia, for example, may be referred to the lower part of the abdomen, so that it suggests appendicitis. Abdominal pain is sometimes induced by pericarditis or spinal caries, while the reference to the knee-joint of the pain of a tuberculous hip is well known, the connection being established by the obturator branch of the lumbar plexus.

Anterior Fontanelle.-Among the clinical points of diagnostic importance that can be ascertained only in the very young, is the condition of the anterior fontanelle. The fontanelle is open until the second year of life, and in this we obtain a valuable opportunity of finding out the condition of the circulation. The pulse at an infant's wrist is so small that little information can be derived from it, and, as an index to the state of the vascular system, it is surpassed by the opening in the skull, the tenseness of which varies with changes of blood-pressure. In some cases the fontanelle discloses the intracranial condition, and can thus be of great service in diagnosis. We have already mentioned that a normal or flaccid fontanelle is not usually associated with any form of meningitis, and the same remark applies to hydrocephalus. It is of no rare occurrence for the 
rapidity of development of a rickety infant's head to suggest to the parents the fear of "water on the brain," and medical advice is not infrequently sought on this point. If, however, the fontanelle is neither tense nor bulging, the fear of existing hydrocephalus can hardly be entertained.

Weight and Growth.-The value of observations on the weight and rate of growth has already been insisted upon. We may here note the additional help that may be afforded by any irregularity in the natural sequence of development. Delay in the co-ordinating power that enables an infant to balance his head, to sit, or to walk; backwardness in cutting the teeth or in talking; each of these is a broad hint to us in our diagnosis.

Deformity of the Chest.-The juvenile skeleton, which is relatively soft, is peculiarly liable to distortion by pressure, so that some diseases attract attention by the bony deformities they induce. Rickets, by producing bowed legs, is easily recognised, but diseases even of the heart or lungs may betray themselves in their effects on the neighbouring bones. An enlarged heart will find space for itself by thrusting forward the ribs. This localised bulging should always excite suspicion of valvular disease. The presence of a pent-up collection of pus, as in an empyema, moulds the chest of its side until it may be obviously larger than the opposite side. A fibroid lung, congenital atelectasis, or obstruction to a bronchus will affect the shape of the corresponding half of the chest, but in these examples the diseased side will be the smaller in circumference.

If the thorax is to develop normally, the freeway from the nose to the lungs must be unobstructed; but when the airpassages are partially occluded at any level-whether by pressure of an enlarged gland or an abscess, by adenoids, or by any of the other affections incident to these parts-some degree of deformity of the ribs is likely to follow. In acute cases, such as laryngeal diphtheria, the alteration may become marked within the space of a few days. These few examples will be enough to show the value of a careful inspection of the configuration and symmetry of the thorax. In adults, where the ribs are stronger and better able to resist extraneous force, 
this help in diagnosis is less often obtained-a point which is probably responsible for the comparative inattention bestowed on the condition in children. A series of records of changes in the shape or volume of the chest in special cases is sometimes of service.

Alterations in the normal shape of the chest wall may be responsible for secondary changes elsewhere, the cause of which may be erroneously interpreted if the thoracic deformity is overlooked. This malformation not infrequently causes the heart to be pushed out of position, so that its impulse beats to the left of the usual site. This might suggest at first the possibility of an hypertrophied ventricle. Rarer, but very interesting, is the existence of clubbed fingers as the result of thoracic deformity. Another effect is an unusually prominent abdomen. The swelling is likely to attract the attention of the least observant of parents, and advice is often sought on this account. In some cases a suspicion of tuberculous peritonitis may be raised, especially if an additional symptom such as diarrhœa is present. Yet frequently this condition results merely from a thoracic deformity which, lessening the capacity of the chest, forces the liver and stomach to a lower level, with the result that the abdomen becomes prominent.

Abdomen.-As regards the diagnostic value of abdominal examination, the two difficulties that so often interfere with the investigation in adults-excess of subcutaneous fat and strong abdominal muscles-are of little account, and the examination is thereby made much easier. Apart from the discovery of tumours, the general sense of resistance of the abdomen is sometimes suggestive. Experience alone, however, can teach the distinctions between the "doughy" feel of tuberculous peritonitis, the elasticity and resilience of flatulence, and the firmer sensation of ascites. We must further remember that just as a deformed chest may produce a swollen abdomen, so abdominal tumours may expand the lower ribs or displace the thoracic organs.

Tonsils. - The throat is often of greater diagnostic importance in children than in adults, and the explanation of many 
acute symptoms may be found with the help of a tonguedepressor. Apart from scarlatina and diphtheria-in each of which the relation of the tonsillitis to the general disease is usually sufficiently obvious-acute rheumatism is one of the most frequent causes of sore-throat in childhood. In this disease the tonsillitis is an early symptom. Often, indeed, it is the only symptom except for a mild degree of fever. Follicular tonsillitis, which is by no means uncommon, is associated with more constitutional disturbance and greater glandular enlargement than the rheumatic form.

Nearly all the items we have been discussing tend to facilitate the diagnosis of children's complaints. We must now turn to an important series of conditions which counterbalance these advantages, and which, though of the greatest diagnostic value in adults, are of relatively less importance in the very young. They include such important clinical indications as temperature, pulse, respiration, expectoration, vomiting, diarrhœa, rigors, etc.

Temperature.-The younger the patient, the smaller is the diagnostic importance of such cardinal points as temperature, pulse, and respiration. Their significance, too, differs in the cases of adults and children. The equable temperature that is characteristic of mature years is deranged only as the result of fairly severe constitutional disturbance. An addition of two or three degrees Fahrenheit usually indicates some definite illness, and has a marked effect on the individual. Heatregulation in children has by no means attained this constancy, and very slight influences produce marked elevations of temperature. It is a matter of common knowledge that a trivial digestive disturbance, even a single improper meal, may increase a child's temperature by several degrees. As might be anticipated, this instability is associated with comparatively mild constitutional symptoms. Children with febrile temperatures often experience no discomfort or malaise, and may preserve their customary energy unimpaired. A boy of nearly five, who attended my out-patient department at the Evelina Hospital, was found to have a temperature of more than $104^{\circ} \mathrm{F}$., 
yet so little incommoded was he that shortly afterwards he was seen to join in play with other children in the waiting-hall.

Many examples could be given of accessions of temperature of two, three, or even four degrees, which produced only a slight effect, yet it would be wrong to conclude that temperature is a negligible point in diagnosis. A sudden increase of temperature is one of the first signs of an infectious fever, of pneumonia, and of other acute illnesses, and for this reason it always requires serious consideration. Similarly, a subnormal temperature, especially if it occurs during the course of pyrexia, may indicate a serious change in the child's vitality. The comparative indifference with which children can tolerate temporary elevations does not enable them to resist a prolonged fever, which dangerously saps their feeble powers of endurance. Lastly, it must be remembered that a febrile period will often leave the heat-regulation even less stable than before, and for some days or weeks a trivial disturbance such as tears, the application of a surgical dressing, or, what is the common experience in a children's ward-visiting-day, is followed by an elevated temperature.

Pulse.-I have pointed out earlier that the examination of the radial pulse of a young infant is not of great moment. Its limited importance is partly due to the fact that the artery is so small that its alterations cannot be appreciated by the crude examination of the finger-tips. In addition to this drawback, we must remember that those changes in the pulse which are so commonly encountered in adults are exceptional in children. Abnormally high blood-tension is rare at this age, as are thickening and rigidity of the coats of the artery or tortuosity of the vessel's course. Toxic arhythmia, aortic regurgitation, and aneurisms-three frequent causes of an abnormal radial pulse in adults-are hardly known in early life. With infants the condition of the circulation must be ascertained, as already mentioned, with the finger on the anterior fontanelle, supplemented, if necessary, by the stethoscope at the heart.

Neither rate nor regularity of pulse has the same importance in childhood as in adults. In the former the rapidity of the 
heart-beat is extraordinarily sensitive to stimulations which reach it from without, and very slight causes may entail a marked variation in its speed. The mere act of sleeping notably retards the heart. As a result of this susceptibility to reflex impressions, or of an intrinsic feebleness of rhythm, the hearts of many young children, if listened to for some little time, will be found to beat at unequal intervals. Such irregularity in adults is rightly viewed with disquietude, but in the young it is of little significance. Yet experience will show that this statement needs qualification, for in some diseasesespecially in meningitis-the irregularity of the heart-beat is an important diagnostic sign, and it has a similar value when associated with any indications of heart-disease. As a general rule, the halting character of the rhythm is more pronounced, and continues over longer periods in meningitis and in heartdisease than in the normal irregularity of a healthy heart.

Respiration.-The associative connection that exists between pulse-rate and respiration-rate suggests the possibility of an irregular periodicity in breathing, and it will be found that the placid rhythm with which the adult fills and empties his lungs has not developed in the young. In the chapters on the physiology of children it was mentioned that Cheyne-Stokes breathing was of normal occurrence in babies. Though this phenomenon becomes less frequent with advancing age, yet the rhythm of breathing is easily disturbed throughout childhood. Pauses may be made, or quickened breaths taken without obvious reason. The sensitiveness of a child's respiratory mechanism to outside impulses exposes its ordinary quiet breathing to frequent disturbances which are sometimes of a curious nature. Thus the administration to young infants of either chloroform or the A.C.E. mixture often modifies the rhythm so that the inspiratory movement is duplicated, and two short crowing inspirations in rapid succession precede each expiration.

In disease, respiration is often very strikingly altered, and, as a general rule, any marked and persisting change in its rate, type, or regularity is a valuable diagnostic sign, especially in the earliest years. A marked acceleration of breathing should raise 
strong suspicion of acute lung trouble-pneumonia, bronchopneumonia, or bronchitis-even in the absence of confirmatory signs on auscultation. It is of common occurrence, for example, for pneumonia to affect the respiration-rate some hours or even days before the inflammation can be localised with the stethoscope, and the same occurrence is met with in bronchopneumonia. If, in addition to the rapid breathing, the nostrils are seen to dilate and fall, there can be no reasonable doubt that an acute respiratory trouble is present. Many observers have called attention to the curious modification in breathing that takes place in children, especially those who are the subjects of acute broncho-pneumonia. The natural rhythm of health allows a pause after each expiration, but none between inspiration and expiration; but in this modification the rhythm changes, so that the pause separates inspiration from expiration. As the expiration is generally accompanied by a grunt, this "inverted" type can be readily observed. Its diagnostic value is great, though I have never convinced myself that it is not as common in other severe forms of lung-disease as in bronchopneumonia.

Yet another type of altered breathing is seen when the abdominal respiration which is normal in children is supplanted by thoracic respiration. The latter is unusual except as the result of disease, which makes free movements of the diaphragm painful or impossible-e.g., pleurisy or peritonitis-but if both varieties are in vigorous action simultaneously some acute respiratory affection is usually indicated. Mention should be made here of a mistake which sometimes occurs when the diaphragm is paralysed after diphtheria. Breathing is then carried on by thoracic movements, but the fact that the abdomen still rises and falls may obscure the real condition; yet closer inspection will show that the abdomen rises with expiration and falls with inspiration-a reversal of the normal sequence.

Apart from Cheyne-Stokes breathing and the healthy interruptions mentioned above, irregularity of rhythm is specially noticeable in diseases of the brain or its membranes, and in these cases it may be a valuable sign. It often develops 
towards the close of many fatal conditions, when it is the last flickerings of a vital function. On the other hand, marasmic infants may breathe in an irregular fashion even though their end may be hours or days off.

A special modification of respiration that requires mention is stridulous breathing. Inspiratory stridor is by no means uncommon in infancy, and it is associated with various conditions, many of which are serious. In some cases its onset is sudden and its duration temporary. In other cases it is chronic. Perhaps the best known of the acute forms is that which is associated with rickets-namely, laryngismus stridulus. The attack, which generally occurs without warning, begins with a sudden inhibition of breathing, so that the face and lips become livid, and it terminates with a long-drawn, crowing inspiration. Laryngismus stridulus is sufficiently characteristic to offer very little difficulty in diagnosis. The other forms of acute stridor are, however, more easily confused. They occur in connection with post-pharyngeal abscesses, laryngeal diphtheria, foreign bodies in the air-passages, and acute bronchitis. We are not likely to confuse the first named with any of the others if we take the precaution of examining the pharynx, both visually and digitally. With laryngeal diphtheria, the history of the case, the general condition of the child, and the occurrence of albuminuria are usually sufficiently suggestive even in the absence of a bacteriological examination. In the case of foreign bodies, the cause of the stridor may be less easy to recognise, partly because the clinical history is often defective, partly because the auscultatory signs may not be distinctive. Any suspicion that arises as to the presence of a foreign body should be confirmed with the help of the X-rays. Lastly, stridor may occur in cases of severe bronchitis. This cause is, as a rule, easily identified by the signs on auscultation, and by the negative indications of diphtheria or postpharyngeal abscess.

Persistent stridor, lasting for weeks or perhaps months, is generally due to one of four causes. Sometimes it is congenital, appearing at or shortly after birth, becoming much more marked on exertion-as in crying-but often clearing up 
altogether in the second year. In other cases the difficulty of breathing is due to enlarged glands pressing on the trachea or bronchi. This condition often occasions suggestive signs in one or both lungs. Sometimes a marked inspiratory stridor is produced by chronic laryngitis. As a last cause, we may mention intralaryngeal growths. These are most commonly of a papillomatous nature.

Expectoration.-Since children do not expectorate, their sputum is rarely available for our inspection or for microscopical examination. Even in those diseases in which excess of mucus is coughed from the lungs, the secretion is at once swallowed. The help that we would gain from noting its appearance or the presence in it of blood or tubercle bacilli, has therefore to be foregone, except occasionally when children who have reached an age of sufficient intelligence can be persuaded to expectorate.

Vomiting.-Vomiting is perhaps one of the most frequent concomitants of disease in childhood, and therefore its importance will vary in different cases. In nurslings it is often merely a regurgitation of surplus milk shortly after a full meal, and is no more than a physiological act. It may be caused by reflex irritation, while recurrent attacks associated with acetonuria have been described as a special disease in children from about six to twelve years old. As an indication of digestive trouble or of unsuitability of diet, this symptom acquires its greatest importance in infancy. The diagnosis is materially assisted by observing its relation to feeding, and also by noticing the characteristics of the vomit. In somewhat older children it is a not infrequent symptom at the onset of the acute febrile diseases, and on these occasions it may be severe, but is not usually persistent. The same symptom occurs in cases of intussusception, of tumours of the brain, and of inflammation of the meninges. Other conditions in which it may be a prominent feature are acute pericarditis, whooping-cough, and migraine.

Constipation.-Constipation, though by no means rare even in earliest infancy, is not often symptomatic. It is rather a clinical entity than a sidelight on a larger trouble, and its importance is therefore not great. It has, however, earned the 
reputation of being one of the most fertile causes of the minor disturbances which affect the average child's health, and there can be no doubt that many febrile and other attacks subside after a purgative has been administered. To what extent and how often this sequence is an instance of "post hoc, propter hoc" cannot, unfortunately, be settled. Constipation that obstinately resists the usual remedies should attract attention to the condition of the large bowel which, either by its chronic dilatation, by some congenital narrowing, or by a fissure in the rectum, may provide the reason for the condition. Stoppage of the bowels, if associated with vomiting, is always a serious matter. This combination of symptoms is found in cases of meningitis, of intestinal obstruction-usually intussusceptionand of peritonitis.

Diarrhaa.-In diarrhœea we have a symptom of considerable value. The great frequency of intestinal disorders in childhood places this phenomenon among the commonest symptoms. It is the key to many an abdominal problem, partly because of the ease with which its presence is recognised, and its intensity and variations estimated, partly because of the ocular demonstration it affords of processes at work out of sight. Whether acute or chronic, it is generally due to the condition of the intestinal contents, of the walls of the bowel, of the mesenteric glands, or of the peritoneum. As an indication of disease outside the abdominal cavity, it is rare except in empyemata. Diarrhœa is not likely to lead to an erroneous diagnosis, if only the doctor takes the simple precaution of personally examining the fæces. Apart from epidemic gastro-enteritis, the acute forms most commonly result from irritating, indigestible food, without any definite pathological lesion-the very large majority of cases-from acute entero-colitis, acute colitis, or from acute intussusception. In the last named, in which the evacuations consist of blood and mucus only, the presence of an abdominal tumour would be conclusive. In colitis and entero-colitis, blood is often mixed with the fæces, the temperature is usually raised, and the abdomen is swollen and tender. In simple acute diarrhœa, undigested food that has been hurried along the intestines may be found in the earlier motions. 
The diagnostic problem with chronic diarrhœe is its possibly tuberculous origin. A correct opinion is often formed with difficulty, especially as emaciation and a swollen abdomen occur both in the tuberculous and in the simple chronic forms. By remembering that these two symptoms are not distinctive of the former variety, we shall avoid labelling many cases of persistently loose bowels as tuberculous.

The importance of the two minor symptoms, epistaxis and rigors, varies in early and in adult life.

Epistaxis is of very frequent occurrence among children, and is specially liable to occur spontaneously, or at any rate without obvious reason. It is not uncommon in hæmophilia and other blood diseases. It occurs at or soon after the onset of whooping-cough, typhoid fever, and pneumonia. It arises during the course of Bright's disease, orthostatic albuminuria, and thrombosis of the cerebral sinuses.

Rigors are notably rare in infants and are much less common in children than in adults. Their great diagnostic value in conditions where the presence of some deep-lying abscess is suspected cannot be insisted upon in children in whom purulent collections less often give rise to this symptom. Thus, both in empyemata and in appendical abscesses, rigors are the exception. There are but three or four diseases in the course of which they occur with any frequency. In acute pyelitis they are stated to be frequent; in lateral sinus thrombosis they may be repeated; while in typhoid fever and in pneumonia they may occasionally be noted.

Diagnosis of Diseases of the Chest.-Having now dealt with the more important symptomatic peculiarities of children, we can pass to what is generally admitted to offer the greatest difficulty of interpretation-namely, the auscultatory signs of pulmonary disease. These signs are so dissimilar in children and adults, that accuracy of diagnosis requires long experience. Special attention will therefore be bestowed on the peculiarities of the young in this respect. Before we deal with these points, however, a few words are required in connection with the heart.

The natural irregularity of the heart-beat has already been 
mentioned, but we must further recognise that the position of the cardiac impulse is variable. It may be in the nipple-line or even beyond it without denoting disease. It is readily displaced by pressure or traction from neighbouring parts, especially by a deformity of the chest, fluid in one pleural cavity, or a collapsed or fibroid lung. If the abdomen is distended by gas, ascites, or a tumour, the heart may be pushed up out of its usual position. In endocarditis and pericarditis the interpretation of the signs is usually sufficiently easy. Owing to the thin covering of a child's thorax-which is all the thinner since heart-disease is a wasting disease-excessive force or diffuseness of the heart-beat is revealed with peculiar distinctness, and these two signs are therefore of much value in recognising and estimating enlargement and dilatation. Præcordial bulging has already been noted and is always a most important indication. For the rest, the details obtained by the physical examination of the heart in children are very similar in character and meaning to those with which we are familiar in adults, though the sounds are transmitted to greater distances in the former. One small point may be noted-namely, that a murmur is occasionally heard in children beneath the clavicle, especially the right, but appears to be of no clinical importance.

Pulmonary auscultation in the young presents many pitfalls. The first of these is that the peculiarities of their healthy vesicular murmur are not always sufficiently clearly recognised. Consequently, abnormalities may be overlooked or normal sounds regarded as indicative of disease. The healthy breathsounds constitute the so-called "puerile" breathing and possess two cardinal features. They are more highly pitched-but not necessarily louder or coarser-than in adults. The expiratory murmur is more prolonged and therefore more distinctly heard. In other words, the murmur normal to children presents to some extent the qualities of bronchial breathing as heard in adults.

How this resemblance is the frequent cause of confusion may be illustrated by the signs produced in acute lobar pneumonia - say, at one apex-though a similar difficulty arises with fluid in one pleural sac. Over the consolidated lobe the entry of air 
will be slight, and the vesicular murmur, even though bronchial, will be unusually faint and distant. Compensatory breathing, which is common in children, becomes established on the opposite side, where the puerile murmur becomes correspondingly louder and, to the casual ear, not unnaturally suggests loud bronchial breathing. When this is contrasted with the feeble sound of the diseased side, the observer may be tempted to identify the healthy apex as pneumonic and the consolidated apex as normal. Yet when the ear has become more nicely attuned to fine distinctions of sound, it will recognise in the feeble murmur, soft though it is, the qualities that make up true bronchial breathing.

In cases of basal pneumonia, a slight increase of resonance and of vesicular murmur at the apex of the same side is a not uncommon result. When this is contrasted with the healthy condition of the opposite apex, a possibility occurs that the latter, with its relative dulness and fainter murmur, may be regarded as the site of the pneumonia.

Another difficulty arises from the fact that the two sides of the chest may move unequally. One lung expands more freely than the other, and the loudness (though not the quality) of the vesicular murmur on that side is increased. Sometimes a portion of one lung seems temporarily to undergo less expansion than neighbouring parts-the result perhaps of some obstruction to its contributory bronchial tube-and the vesicular murmur over this disused area is damped or even abolished. As this change may be associated with dulness on percussion due to absorption of the contained air, it will readily be seen that the counterfeit signs of pleural fluid are produced.

A third difficulty is found in the fact that signs in one lung, especially those of consolidation, are peculiarly liable to be transmitted to the opposite side. Though this condition would not result in the affected area being overlooked, it may sometimes lead to doubt as to whether the pneumonia is double.

Pleural effusions are probably never more ready to disguise themselves under equivocal signs than in the earliest years of life. The wholesale negation of the auscultatory properties of healthy lung-tissue that characterises the condition in the full- 
grown chest is inconstant in the young, where an effusion may be represented by signs compatible with pneumonia, phthisis, or fibroid lung. A frequent result of fluid at one base is to leave the vesicular murmur over its limits unaffected, but to induce loud compensatory breathing on the opposite side. Here, by the same confusion as was instanced above with an apical pneumonia, the signs on the healthy side may be interpreted as abnormal. In other cases, the effusion, still leaving the vesicular murmur over it unchanged, either exaggerates the tubular character of the breathing at the same apex or causes definite bronchial breathing. Either alteration may suggest apical pneumonia or phthisis.

A special difficulty in distinguishing pleural effusions from pneumonia arises when extensive consolidation of the lower part of the lung produces not only dulness over the inferior lobe but a skodaic note at the apex-a condition which is usually characteristic of effusion. Inasmuch as the vesicular murmur is sometimes absent over solid lung, and bronchial breathing is sometimes present over fluid, the nicety of the diagnosis will be understood.

It is well to remember that pulmonary signs in children are subject to frequent variation, and therefore more than one examination may be desirable before making a diagnosis. Merely slight differences, whether in percussion or auscultation between corresponding areas on the two sides, should be regarded cautiously, and not too much weight attached to them. In all cases the results of auscultation must be combined with other physical signs of well-established value (such as the character of the respiration, percussion notes, displacement of viscera, inequality of the two sides of the chest, impaired movement of ribs), and the whole then considered in association with the general clinical facts. 


\section{CHAPTER XV}

SOME POINTS IN THE TREATMENT OF SICK CHILDREN

Preventive Treatment : Cleanliness ; Food ; Sleep ; Exercise ; Clothing. -ACUTE Illness-Chronic Illness-Medicinal Treatment: Dosage ; Susceptibility to Drugs; Alcohol ; Tonics; Pharmaceutical Preparations suitable to Children-SPECial Remedial Measures: Methods inapplicable to Children; Baths; Antipyretic Remedies; Sponging, etc.; Rectal Injections; Nutrient Enemata; Medicinal Enemata; Aperient Enemata ; Irrigation of Colon; Gastric Lavage ; Nasal Feeding-INFANT FEEDING: Feeding of Healthy Infants; Natural Feeding; Wet-nursing; Artificial Feeding ; Proprietary Foods; Effects and Treatment of Improper Feeding; Wasting; Diluents of Milk; Gastro-intestinal Disturbances; Substitutes for Cow's Milk.

PREVENTIVE treatment is of the first importance in the case of children's diseases. Much of the suffering in infancy and in later years is due, as we have seen, to ignorance of the essential requirements of early life, and the young are not likely to secure their full chance of attaining healthy adolescence until a better understanding of the conditions that breed disease has been diffused through all levels of society. Probably the day is not very distant when the wisdom will be recognised of submitting the young as a matter of course to regular medical supervision; and parents will come to regard this step as not the least valuable form of life assurance. Already the precaution is taken among the better classes with respect to their children's teeth, and a logical expansion of this procedure will lead to the employment of medical science not merely to regain health but to maintain it.

The principles that govern preventive treatment must be 315 
founded on the facts that have been set forth at length earlier in this book. The dependence of growth and development on nutrition makes the question of diet of greatest importance, and the concluding pages of the present chapter will be devoted to a succinct account of this subject. The overwhelming prevalence of bacterial diseases in childhood renders cleanliness in every detail of their lives essential. Not only is cleanliness of the body and the clothing required, but also of the nursery, and of all who come in intimate contact with the infant. Especially important is it to exclude every risk of tuberculous contamination, whether in relatives or friends, garments or floor.

Next in importance to good and proper food and scrupulous cleanliness comes the judicious alternation of rest and exercise. The necessary hours of sleep have been recorded on p. IO4. Exercise implies not only muscular activity, but the use of all functions, including those of the special senses and of the brain. In addition, light, sunshine, and ventilation must not be neglected, while warmth and clothing must be carefully adapted to the susceptible requirements of early life. When the age of schooling has been reached new dangers arise, and to meet these the precautions already dealt with in Chapter V. must be taken.

With these few remarks on prevention, the special subject of this chapter-namely, the remedial treatment of disease-can be approached. The general treatment of acute diseases will be first dealt with, after which the treatment of the more chronic affections will be discussed. Following this will be some observations on medicinal treatment, and on therapeutic remedies especially applicable to children.

Acute Illness.-Wherever practicable a child that is stricken by one of the acute illnesses should be at once placed under the care of a trained nurse. On occasions of danger, urgency, or unpleasantness, a mother is not always able to act with the requisite decision or firmness, and her child's life may therefore be unnecessarily hazarded. The frequency and suddenness of unexpected complications are additional reasons which render the constant presence of a skilled attendant highly desirable. It need hardly be said that the services of a nurse who has been specially trained at a children's hospital will be of greater 
value than those of one with adult experience alone, and every attempt should be made to secure the former.

The distinguishing points of acute illness in childhood being, as we have seen, its irregular course, quick fluctuations, sudden relapses, and as sudden recovery, each case must be followed with the closest attention, and the first intimation of a change in condition watched for with the utmost care. Every alteration for the worse must be at once met with such necessary modifications in treatment as will act efficiently and with dispatch. These measures should be continued only so long as the condition to which they were opposed persists. Should a means at first adopted fail in its anticipated effect, time must not be wasted, but fresh resources hurried up. Children in a crisis often let their powers of resistance tumble to pieces. Yet, on the other hand, we know that they will sometimes pull themselves together when things seem blackest; and therefore treatment should never be given up because the day seems lost. This hopefulness is the more necessary since children assume with comparative readiness the appearances of the gravest conditions, yet without serious cause. Since the constitutional disturbances which are common to many diseasessuch as high fever, rapid pulse, convulsions, delirium-may not tell the same tale as in an adult, we should be chary of employing any vigorous treatment aimed at these symptoms alone. As a rule, drastic remedies and potent drugs are not suited to children, and mild measures will generally be efficacious.

Finally, it will be remembered that in convalescence from an acute illness, a child's spirits shake themselves free of their invalid trammels before the pathological effects have cleared away, and therefore at this stage "festina lente" should be the doctor's motto. The unavoidable frequency of complications and sequelæ enforce this caution, for should any such trouble arise after the child has been allowed premature freedom from restraint, the blame, not unnaturally perhaps, may be laid on a too sympathetic treatment.

Chronic Illness. - In the chronic forms of illness, treatment, following the diagnosis, will be directed first to rest the function or part impaired; next to counteract the secondary changes set 
up by the morbid process and to supplement the deficiencies arising therefrom; and lastly, to support all other functions. It is, as a rule, unwise to attempt to stimulate any temporarily impaired function, for its own recuperative power is far more efficient than any applied remedy, and it acts to best advantage under the stimulus of rest. Unfortunately, children with their active habits and their intolerance of restrictions, often object to the enforced passivity of any function. This difficulty applies not only to muscular rest, but to digestive or circulatory rest. In such matters they are too young to appreciate the wisdom of curbing present desire in order to escape future trouble, still less of submitting to present discomfort in order to ensure future well-being. With them, therefore, a strong lever is lacking by which the physician is able to keep his older patients on the narrow way that leads direct to recovery. As soon as unpleasant symptoms, pain, or discomfort are smoothed away, the child, happy in forgetting trouble, is as likely as not to repeat his original indiscretion. In matters of injudicious eating, exposure to cold, sudden muscular effort, excitement, and the like, children have short memories and gather little experience. Discipline and parental authority are not always sufficient to ensure the requisite abstention or rest, and often we have to devise special means to secure our end. These expedients should be of such a form that their raison d'être is not apparent to the child-who will therefore not feel the wholly childlike desire to do that which it sees should not be done-or they should be associated with some interest that appeals to its particular fancy or enjoyment. As an illustration may be cited the useful practice of attaching side-splints projecting beyond the feet to the legs of a rickety child to prevent it walkinga device more efficient than any injunctions or supervision, and not resented, seeing that the patient does not, as a rule, appreciate its object.

In those very common cases where, without any definite disease, though often as a result of preceding acute illness, a child sinks into a condition of general debility, losing his vigour, brightness, and appetite, ceasing to gain in weight, or perhaps actually wasting, medicinal treatment concedes rank to general 
measures for improving the health. The appetite must be tempted by palatable food temptingly served. Such regular exercise should be arranged as will induce a desire for nourishment, and an atmosphere should be selected to brace the child's constitution. Generally speaking, the repair of health would be more rapid if the dietetic tastes and idiosyncrasies of each child were consulted a little more than is sometimes the case, and articles of food excluded or pressed less in accordance with traditional views of what children ought to eat than with what they desire.

The advantages of a change in surroundings should not be forgotten in the treatment of these cases, for the good that comes of a country or seaside holiday is often remarkable. Nervous, irritable children will profit more by some such change of scene and atmosphere than by any other treatment, and it is much more desirable than sedative drugs. Marine climates, which are cool and bracing, are to be preferred; while warm, humid neighbourhoods are usually unsuitable. In this country, the East coast, from Margate to Scarborough, presents numerous towns and villages well suited to children. On the Continent, the bracing shores of the English Channel, the North Sea, and the Baltic Sea are in similar repute; in France, the dry, sandy coast running south from Boulogne, past Berck and Paris Plage; in Belgium, Blankenberghe; in Holland, Scheveningen and Zaandvoort; in Germany, Ahlbeck, Swinemünde, Kolberg, etc.

\section{Medicinal Treatment.}

The medicinal treatment should be conducted on the simplest possible lines. It is rarely necessary to invoke the aid of any drugs except those whose physiological actions are well established, and no medicine should be administered without a definite object in view. The day has fortunately passed when the efficacy of a child's draught was supposed to vary inversely as its palatability, and a nauseous or unpleasant medicine should never be ordered if the desired end can be attained by a less disagreeable prescription. ${ }^{1}$ Posology is to

1 The physician is well advised who never writes a prescription for a child without first having sampled the medicine himself. 
be carefully studied, and only a sufficient dose ordered to effect the purpose in mind. The rapidity with which the state of a child will alter makes it highly undesirable to allow any surplus, as it were, of a drug in the circulation, lest its influence should remain after the condition that called for its exhibition has passed. Forgetfulness of this principle may lead to a total effect that is harmful rather than beneficial. Again, certain drugs-for example, salicylic acid, arsenic, the bromides, atropine, ipecacuanha - may produce, with undesired prominence, their subordinate actions more strikingly than the primary effect for which they were employed. For these reasons a small dose repeated at short intervals is often preferable to more liberal medication. The principle has a further advantage, inasmuch as both absorption and excretion are active in the young, and the risk of over-rapid entry of a drug into the system, or of intervals when the last dose has been eliminated, and the next is not yet due, will be avoided. The application of this last factor to the administration of medicines during the night hours should not be overlooked. Allowance should also be made for drugs returned by vomiting soon after administration.

It is well known that children are tolerant of some drugs, but peculiarly susceptible to others. Among the former are belladonna and its derivatives, the bromides and the iodides, chloral, quinine, camphor, alcohol, calomel, and all mercurials. The latter include opium, codeine, morphia, apomorphine, jaborandi, phenazone, phenacetin, and acetanilide, which act with special potency, especially in infants of a few weeks or months. Even with the drugs of the first group, it is only prudent to begin with a dose well within the margin, increasing it later as the ground is proved secure. An average dose can be readily ascertained by the well-known formula:--adult dose multiplied by $\frac{\text { child's age }}{\text { child's age }+ \text { I2 }}$. Thus the dose for a child of four would be $\frac{4}{4+12}=\frac{1}{4}$ of the adult dose.

When prescribing opium, we must be specially careful. The tincture, the compound tincture of camphor and Dover's 
powder are the preparations usually employed. The dose of the tincture, as given by Goodhart and Still, can be easily remembered-namely, $\frac{1}{4}$ of a minim at $\frac{1}{4}$ of a year, $\frac{1}{2}$ a minim at $\frac{1}{2}$ a year, and $I$ minim at $I$ year. These doses, halved, represent the quantity in grains for Dover's powder (i.e., $\frac{1}{8}, \frac{1}{4}, \frac{1}{2}$ of a grain at 3,6 , and 12 months respectively), while of the compound tincture, 3, 6, and 9 minims represent the doses at corresponding periods. A safe method of placing a young child under the influence of opium is to dilute the tincture with excess of water and to give a dose corresponding to the dilution at intervals of half-an-hour or an hour.

Alcohol is a stimulant of great value in acute and collapsed conditions, and it has this peculiar value in children, that before its stimulating effect is over the patient will often have rallied more than enough to withstand any possibly depressant afteraction of the drug. As a tonic it is best avoided. Its virtues are not contained in brandy alone, but are possessed by various of the alcoholic tinctures of the Pharmacopoia, especially the compound tincture of cardamoms and the compound tincture of lavender, the latter being the stronger. In whatever form it is administered, it should be given dilute.

Tonics, though of unquestionable value, have limitations which are narrower in children than in adults. It is not without suggestiveness to note that those tonics which general experience shows to be the most efficient are those in which sugar predominates. This observation may be correlated with the known importance of glycogen to growing tissues as well as with the universal liking of children for sugar. These facts are worth bearing in mind, both in prescribing tonics and in framing diets for convalescent children.

The modern pharmacist, by his many delectable preparations, has robbed the prescription of drugs to children of half its difficulty, but a very short experience of young patients will be sufficient to show that many methods of administering drugs to adult patients are not readily tolerated by the young. Solid preparations (e.g., pills) that require to be swallowed whole should be avoided; cachets and capsules are liked but little better. Mixtures probably need less persuasion for their friendly 
reception than any other preparation, but the volume of each dose should be kept as small as possible. Syrups are readily taken, though they have the disadvantage that in hot weather they may turn sour; glycerine or saccharine in place of sugar will do away with this difficulty. Powders form a very convenient preparation, provided their bulk is kept small. An objection to their use is that some tend to cling about the mouth, thus prolonging any unpleasantness of flavour and increasing the risk of vomiting. Compound liquorice powder, for example, is apt to adhere objectionably to the palate, but this result can be simply obviated by throwing the powder on to the surface of a wine-glassful of water a quarter of an hour before bedtime. At first it floats on the surface, but gradually sinks to the bottom, and, becoming well-moistened, is free from all lumpiness. If milk is used instead of water, a somewhat longer time must be allowed for the powder to settle. The use of jam for covering small quantities of powder is of course known to all, but it is open to question whether wholesome articles of food should be associated in the child's mind with unpleasant experiences. Another successful vehicle, especially with infants, is butter. A small lump of this, enveloping, for example, some calomel and placed at the back of the mouth soon melts and is swallowed without trouble. ${ }^{1}$

\section{Special Remedial Measures}

Children are easily alarmed by the methods of the sick-room with which they are unfamiliar, and remedial measures that are

1 Some years ago a lady who, in rearing a large family, had amassed a store of knowledge on the ways of children, acquainted me with her method of powder-giving. As the device is as successful as it is simple, its details may be useful to any reader to whom they are unfamiliar. She used to take two chocolate creams, one large and one small, and by the sweep of a knife, removed the bottom from the larger. Into its creamy interior she pressed the powder, and, the bottom replaced, the join was smoothed over. At night whichever child stood in need of a mild corrective was given the two sweets, with permission to eat one on the way upstairs, and to make a present of the other to the nurse-an accessory before the fact. Human nature was never at fault. The smaller chocolate reached the nurse and the success of the conspiracy was proclaimed the following morning. 
painful, unpleasant, or elaborate may do harm as well as good. Young patients are rarely able to endure such proceedings either peaceably or patiently, and with every repetition difficulties increase. Intralaryngeal applications, gargling, cauterising, hypodermic injections, venesection, blistering, drycupping, the use of leeches, faradisation, gastric lavage, etc., come readily to mind as measures less applicable to a child old enough to be frightened than to an adult, and, though each or any of them may be necessary at some time or other, they should not be resorted to without special reason. In place of them such simple measures as hot baths, plain or medicated, poultices, fomentations, counter-irritants, spraying, rubbing and passive or resisted movements will be found to possess a charm in allaying troublesome symptoms.

A simple hot bath continued for five to ten minutes or even longer is a most efficient means of reviving an infant collapsed from any cause, or of inducing sleep in a restless child. A hot medicated bath, such as a mustard-bath, possesses a rubefacient action in addition, and should be employed for the special purpose of stimulating the skin. It is most serviceable in severe forms of collapse, or in conditions such as convulsions, where general counter-irritation is desired. Directions for the preparation of mustard-baths vary in different books, but the precise strength is relatively unimportant, provided the desired effect is known and watched for-namely, a pink flush of the skin. As soon as this change has become apparent, the bath has been continued long enough. Local applications of warmth in the form of poultices, or preferably fomentations, are of great service. In applying them it should be remembered that an infant's skin will not stand much heat or irritation, and that bandages are apt in a short time to chafe or even cut the skin. Jackets of wool or of Gamgee tissue are particularly useful and comforting.

Incidentally, it may be mentioned that the application of too much warmth may lead to a marked elevation of temperature. A single hot water-bottle in an infant's cot may cause a rise of three or even more degrees Fahrenheit.

Of the special measures that are in common use in adult 
practice, many are equally applicable to children, but a few may be mentioned here as being of special service in connection with the young.

It is often necessary to reduce an excessive temperature, and such methods as cold sponging, ice-bags, cold pack, cold bath will occur to all. The last mentioned is, with rare exceptions, an undesirable procedure by no means free from the danger of sudden collapse. This objection holds, though to a less extent, with the cold pack. Ice-bags are comparatively harmless, but, as a rule, a feverish child is a restless child, and is likely to part company with the bag. Cold sponging, or rather, tepid sponging, is useful, and, if the body is exposed in quarters at a time, rarely gives occasion for anxiety. Most of these methods, however, necessitate disturbing the patient, and are often resented. The simplest and quickest method to lower an excessive temperature is to inject a few ounces of warm water or saline fluid into the rectum. A soft rubber tube, to the end of which a glass funnel is attached, is gently passed high into the bowel, and from one to four ounces (according to the age of the child) of the liquid at a temperature of $90^{\circ} \mathrm{F}$. is allowed slowly to flow into the rectum. The tube is withdrawn and the operation is ended. Sometimes the whole procedure can be carried through without disturbing the child even if it is sleeping. The injection soon lowers the temperature and possesses the further advantage that it is absorbed, thus adding to the fluid in the circulation and slaking thirst if it is present. Should the temperature rise again the injection can be repeated, this time at a slightly lower temperature $-80^{\circ}$ to $85^{\circ} \mathrm{F}$.

At this stage it will be convenient to mention a few points on the utilisation of the rectum for therapeutic purposes. In cases of collapse, especially in acute abdominal and other conditions, the rectum affords an easy means of increasing the fluid in the blood-vessels, and has advantages over subcutaneous injections in that the latter are painful, they readily allow excess of fluid to be administered and the tissues to become waterlogged, while the skin, if too tightly stretched, may slough or the site of the injection may suppurate. The administration of nutrient enemata is not often required except in infancy. 
Their bulk should be kept small (from $\frac{1}{2}$ to 4 ozs.), fatty ingredients such as yolk of egg avoided, and inorganic salts added to isotonic strength. Infants who are taking only water or saline for nourishment will hold their own in a surprising way. Drugs may often be administered with advantage by the rectum, and sedatives, such as chloral or potassium bromide, can be relied on better if given by this method, than by the mouth, where there is the chance that they may be vomited or spat out.

Aperient enemata should be of comparatively large size ( $\frac{1}{4}$ to I pint or more), except those varieties that contain glycerine or castor oil ; their bulk is often almost as important as their ingredients. It is well to remember that an enema of soft soap sometimes results in a perplexing skin rash suggestive of scarlatina. A very useful rectal aperient for infants is made by rolling a piece of yellow soap, the size of a Spanish nut, into suppository shape, and slipping it above the sphincter. Finally, methodical irrigation of the colon is often of great service in certain forms of acute diarrhœea, especially acute colitis and entero-colitis.

The stomach-tube is an instrument particularly useful in some infantile conditions, but its employment, whether for lavage or feeding, is, as a rule, too strenuously resisted by older children to make its use often advisable. The same difficulty holds, to some extent, of nasal feeding. Severe cases of gastroenteritis often respond satisfactorily to lavage of the stomach as well as of the bowel. Many forms of vomiting, the result of improper feeding, of fermentation in the stomach or of congenital pyloric stenosis, are benefited by the treatment. ${ }^{1}$ Nasal feeding (gavage), which in experienced hands is a simple operation, can be carried out on infants without provoking serious resistance, provided the child has his limbs well enveloped in a blanket, and is held comfortably on the nurse's lap. In older patients it may be required in cases where acute faucial inflammation has produced difficulty in swallowing, in post-diphtheritic paralysis, and on occasions when, owing to coma, etc., there is a risk that the patient may splutter his feed into the larynx and broncho-pneumonia may result.

1 A No. Io or No. 12 soft rubber catheter is a convenient size. 


\section{INFANT FEEDING}

At the present day, when there is a tendency to regard the dieting of infants as an elaborate and highly specialised branch of treatment, it is well to remember that in the majority of cases the feeding should be, and is, a simple matter. Both the desires and requirements of infancy are primitive, and only exceptionally is it necessary to resort to the numerous strange and more or less unnatural preparations that from time to time are widely advertised. Two cardinal rules in infant feeding are that the diet must approximate as closely as possible to nature, and that each case must be judged on its own merits, and not treated by rule of thumb. General principles applicable to all infants are without doubt useful and necessary, but the practical physician will be prepared to recognise many exceptions, and to study the idiosyncrasies of each child.

The following short account of the subject is to be taken as supplementary to that given in Chapter II.

\section{FeEding OF Healthy Infants}

\section{Natural Feeding.}

Every baby should, wherever possible, be fed from its mother's breast, and in no other way until it is seven months old. It should then gradually be weaned, the change being completed by the eighth or ninth month.

The earliest bottle-feeds should contain equal parts of pure cow's milk and of water, with a little sugar and cream. ${ }^{1}$ In the concluding months of the first year, baked flour, ${ }^{2}$ or some

1 For exact quantities see p. 329.

2 Recipe for Baked Flour.-Press a quantity of flour into a puddingbasin, cover tightly with a cloth, and boil in a saucepan for eight hours. Remove the outside gelatinous crust, and, having cut up the remainder into small pieces, spread out on a large dish. Place all night in an oven that has been heated during the day, leaving the oven-door ajar. In the morning thoroughly pulverise with a rolling-pin and store in an air-tight tin.

Take one teaspoonful of this flour, stir in a little milk to make a thin paste. Slowly add a quarter-of-a-pint of boiling water, stirring well. Boil gently for a minute and add the measured feed of the infant. 
similar ingredient, should be added, and later an egg or meatbroth may be given. At one year, when the child has probably tripled his original weight at birth, he should be sufficiently advanced to take, in addition, bread and milk, bread and butter, milk-puddings, a simple soup, potatoes, or vegetables. Six months later, fish or minced meat may be added.

Young mothers are often ignorant of the proper way to suckle a baby. During the last few weeks of pregnancy the nipples should be carefully tended, and, if necessary, hardened by cold sponging or by the application of an astrigent solution containing tannic acid or eau de Cologne. For the first day or two after birth, both breasts should be used at each meal, but subsequently they should be given alternately. While the mother keeps her bed, she will require to turn to one side to nurse her child; later it will be more conveniently accomplished by sitting up. When she is about again, she should sit on a low chair, with her foot of the side corresponding to the breast that is to be given raised on a footstool, so as to elevate the knee and thus support the child's head. The infant should be held encircled in one arm, in such a manner that its head is supported by the palm of the hand. The other hand is to be used to place the nipple between the infant's lips, and then by spreading the fingers to hold back the breast so that it does not obstruct the child's nostrils for breathing.

Too often, unfortunately, a child is brought up from the first by hand, as the mother appears to have no milk. Lactation, however, may normally be absent until the fourth or fifth day, and then develop, provided the infant is placed regularly at the breast. During these days it will be sufficient to allow the child feeds of warm water alone, without any artificial food, and thus save it from the risks of the bottle.

Wet-Nursing.-If the mother's milk fails, we should consider the question of wet-nursing before having recourse to artificial feeding. The services of a wet-nurse are much more commonly in request in France and in Germany than in this country, where the very real advantages of the method are too often allowed to be obscured by its possible risks. Yet by 
exercising ordinary care it should be possible to avoid these latter, and to secure for an infant of middle or upper class parents who can afford the expense, the benefits and safety of breast-milk.

The following are the points to be observed in selecting a wet-nurse: (a) She should be healthy, clean, and, on detailed medical examination, free from all signs of disease, present or past; attention being specially paid to indications of phthisis and of syphilis; $(b)$ Her infant must be similarly examined and proved healthy, and the doctor must ascertain that the child submitted to him is, in fact, the nurse's baby ; (c) The woman's breasts and their yield of milk must be satisfactory. The shape and size of the glands is no criterion, but the nipples must be well-formed, clean, and free from fissures. The quality of the milk may, if thought necessary, be ascertained by analysis. Its quantity is to be estimated, not by the breast-pump, which is a fallacious test, but by weighing the nurse's own child before and after it has suckled. An average daily yield of breasts in full activity is between two and three pounds of milk; (d) On the nurse's behalf the doctor should assure himself that the proposed foster-child is not the subject of congenital syphilis.

\section{Artificial Feeding.}

Artificial feeding is never so satisfactory as breast feeding. If for sufficient reason-such as the state of the mother's health, the quality or quantity of her milk-it becomes necessary to bring up an infant by hand, the following details should be observed.

Pure cow's milk, obtained fresh by morning and afternoon deliveries and stored until required in a closely covered vessel in a cool atmosphere, is to be given in regular and measured feeds. The bottle should be boat-shaped, with a rubber-teat attached directly to the neck without any intervening tube, and both are to be scalded in boiling water and soda after each feed. They should be left covered by cold water until next required. The feeds themselves, to which sugar and cream may advantageously be added, are to be given at bodytemperature as ascertained by the thermometer. If too hot or 
too cold, diarrhoea or some other disturbance may result. The sizes of the feeds and the intervals at which they may be given are shown in the following table:-

Table showing the Composition, etc., of Feeds for Infants of Different Weights.

\begin{tabular}{|c|c|c|c|c|c|c|c|c|}
\hline \multicolumn{2}{|c|}{ Weight. } & \multirow{2}{*}{ 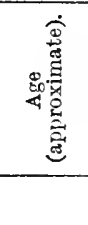 } & \multirow{3}{*}{ 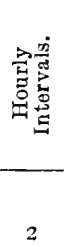 } & \multirow{3}{*}{ 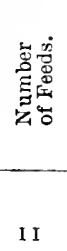 } & \multirow{3}{*}{ 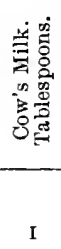 } & \multirow{2}{*}{ 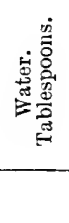 } & \multirow{3}{*}{ 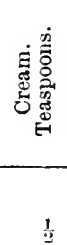 } & \multirow{2}{*}{ 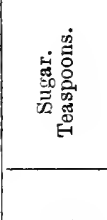 } \\
\hline Kilos & Lb. & & & & & & & \\
\hline 3 & $6 \frac{1}{2}$ & Birth. & & & & 2 & & $\frac{1}{2}$ \\
\hline 4 & 9 & I & 2 & 9 & 2 & 3 & $\overline{\frac{3}{4}}$ & $\frac{5}{4}$ \\
\hline 5 & I I & 3 & 3 & 8 & 3 & 3 & I & I \\
\hline 6 & I 3 & 5 & 3 & 8 & 4 & 3 & I & 2 \\
\hline 7 & I $5 \frac{1}{2}$ & 7 & 3 & 7 & 6 & 3 & $1 \frac{1}{3}$ & 2 \\
\hline 8 & $17 \frac{1}{2}$ & 9 & 4 & 6 & 8 & 4 & $\mathrm{I} \frac{1}{2}$ & I Baked \\
\hline 9 & 20 & I 2 & 4 & 5 & 10 & 4 & $\mathrm{I} \frac{1}{2}$ & jFlour. \\
\hline
\end{tabular}

The foregoing figures are not to be taken as rigidly applicable to the needs of every infant, and the physician will, if necessary, modify them either by increase, decrease, or in their relative proportions, in accordance with his observations of each patient as indicated by the rate of growth and the signs of hunger or of repletion.

It is of no infrequent occurrence for attention to be so concentrated on the size and composition of each feed, that the total amount of nourishment given in the twenty-four hours is overlooked; and it is by no means unknown for feeds to be progressively diluted in a vain attempt to improve the misapprehended state of a hungry child whose only need is a doubled or trebled supply of nourishment. A useful plan in the preparation of a diet scheme is to settle first the total daily amount of milk the child is to receive, and from that starting-point to work out the times, dilutions, and sizes of the feeds. Another common mistake is that of allowing a child to remain too long on a diluted mixture, instead of returning by steps as soon as possible to the normal strength.

By preference children, especially of the poor, should be 
weaned either before or after the hot summer months-not later than the beginning of July or earlier than the end of September-in order to avoid the risk of summer diarrhøea.

After an infant has attained the weight of some fifteen or sixteen pounds, its nutrition is improved by increasing the carbohydrate content of the feed. This may be done quite satisfactorily by the addition of baked flour (see p. 326) until the milk has the consistency of thin cream. A common practice is to make use of one of the more expensive patent foods. These preparations fall into two main groups-those with and those without the solid constituents of cow's milk.

i. Some are based on milk and contain the residue left by evaporating it at an increased temperature and often at a diminished atmospheric pressure. To this powder, which is necessarily expensive, is added varying proportions of carbohydrate (cereal) converted, partially or entirely, into sugar. As starch is not an appropriate food for very young infants, this main group may be subdivided, according as starch is absent or not, into:-

(a) Foods containing dried milk and malted cereal (but no starch).

Examples:-Horlick's Malted Milk; Allenbury's foods, Nos. I and 2.

(b) Foods containing dried milk and only partially malted cereal (i.e., contain starch).

Example:-Nestlé's Food.

ii. The members of the second group contain no derivative of milk, but consist of carbohydrate alone, and these may be subdivided, according to the nature of the carbohydrate:-

(a) Foods consisting of malted cereal.

Example:-Mellin's food.

(b) Foods consisting of partially malted cereal.

Examples:-Benger's, Savory and Moore's, Allenbury's No. 3.

(c) Foods consisting of cereals (i.e., starch).

Examples:-Robbs's biscuits, Neave's food, Ridge's food. 
If a patent food is to be recommended, one should be chosen that is suited to the age of the child, as young infants are not able to digest starch.

The Effects and Treatment of Improper Feeding

Infants fed from the first in accordance with the scheme outlined above rarely need any special modification in their diet. Unfortunately, improper feeding is so common, that frequent illness results, and in these cases special dieting has to be arranged. The customary conditions calling for this alteration are :-

I. Deficient growth (wasting or marasmus, without any other indication of disease).

II. Gastro-intestinal disturbances (flatulence, vomiting, colic, diarrhœa, etc.).

In the absence of organic disease as a cause of these conditions, erroneous dieting will in almost all cases be responsible.

The investigation and treatment of a case should be conducted on the following lines:-

I. Deficient Growth (Wasting, Marasmus), with no other indication of disease :-

A. Food deficient in quantity or quality.

i. Breast-fed.

ii. Bottle-fed.

B. Food sufficient in quantity but imperfectly digested.

i. Indigestible food.

ii. Feeble digestive powers of infant.

II. Symptoms of Gastro-intestinal Disease:

A. Fermentative or putrefactive changes in the alimentary canal.

B. Pathogenic Milk.

C. Improper Food-stuffs.

These classes will be considered in order. 
I. Deficient Growth (Wasting or Marasmus).

A. Food deficient in quantity or quality:-

i. If the child is breast-fed, the mother's milk is at fault. Investigations as to its quality (by analysis) or its quantity (by weighing the child before and after his feeds) should be made. The correct treatment is to supplement-not necessarily to supplant-the breast-feeds with bottle-feeds in accordance with the table given on p. 329 for the particular weight of the child.

ii. If the child is bottle-fed, the conditions of feeding must be revised in accordance with the particulars already given for artificial feeding.

B. Food sufficient in quantity but imperfectly digested.-This may be recognised by examining the stools (or vomit) for milk-curd, the presence of which indicates either indigestibility of food or feeble digestive juices. Curds in the motions may also mean that an excess of food over bodily requirements is being taken-a condition which is easily remedied.

i. Indigestible Food.- Should this be the cause of the wasting, the feeding must be modified until a dietary has been found by experiment that is suitable to the child. The first attempt should be to dilute the feeds, in order that their digestibility may be increased. The diluents in common use are barley-water ${ }^{1}$ and lime-water ${ }^{2}$; less frequently ricewater, ${ }^{3}$ oatmeal-water, ${ }^{4}$ or arrowroot-water. ${ }^{5}$ The relative quantities of these and of cow's milk must be regulated by trial and the proportions revised,

1 Recipe for Barley-water.-Wash two teaspoonfuls of pearl-barley in two or three changes of cold water. Boil the washed barley in a pint of water until two-thirds of a pint remain, and then pass through a strainer. Barley-water must be prepared fresh daily, as it will not keep.

2 Recipe for Lime-water.-Add a lump of freshly burnt lime, the size of a walnut, to a pint of cold water. Place in a corked bottle for twelve hours, when the water will be ready for use.

3 Recipe for Rice-water.-Soak one ounce of rice (previously washed 
if required, at frequent intervals. It is rarely necessary or desirable to continue a dilute mixture if the child takes it readily and with comfort, but an attempt should be made to pass to a stronger preparation.

If dilution fails, recourse must be had to one or other of the temporary substitutes for cow's milk described below (see II. B.).

ii. Fceble digestive powers can be surmised rather than proved. The obvious treatment is to bring the food within the powers of the juices rather than to adhere to a relatively indigestible diet and attempt to increase the activity of the secreting alimentary glands.

\section{Symptoms of Gastro-Intestinal Disease.}

These arise from fermentative or putrefactive changes in the alimentary canal, from soured or pathogenic milk, or from the administration of improper food-stuffs. After detailed investigation of these cases, the principles to be followed are: $(a)$ to stop all probable or possible extraneous causes of the trouble; $(b)$ to allay the symptoms by medicinal and local treatment; $(c)$ at the same time temporarily to restrict the diet as much as possible, in order to rest the disordered viscera; $(d)$ in convalescence to prescribe a diet that will obviate any recurrence while fully satisfying the healthy requirements of the infant.

A. Fermentative or Putrefactive Changes in the Alimentary Canal.-These will be recognised by examining the vomit or motions. The former may be watery, with little or no curd, perhaps frothy from the presence of the gases of fermentation, and it may have a sour and unpleasant odour. The motions are pale or yellow or green, more liquid than they should be, perhaps frothy,

clean in cold water) for three hours in a quart of water kept warm on the hob. Boil slowly for an hour and strain.

4 Recipe for Oatmeal-water.-Add one tablespoonful of oatmeal to a pint of water and allow to simmer gently for an hour.

${ }_{5}$ Recipe for Arroweot-water.-Add two teaspoonfuls of arrowroot to a pint of water and simmer for five minutes, stirring thoroughly. 
and often very offensive. Commonly the epigastrium or the whole abdomen is distended with gas.

As a first step in treatment, it may be necessary to wash out the stomach or bowel at least once to rid them of their decomposing contents. An aperient (magnesium sulphate, calomel, castor oil, magnesia, rhubarb, etc.) helps in the same direction. Later, bismuth salicylate, salicylic acid, salol, chalk, catechu, hæmatoxylin, etc., are all serviceable. Diet will be amended as above.

B. Pathogenic Milk.-Before concluding that the composition of the milk is at fault, enquiry must first exclude the possibilities of the feeds being irregular in time, inappropriate in quantity, or unsuitable in temperature. The source of the milk should be ascertained (so far as possible), the length of time it is kept in the house before use, the place (ventilation and temperature) and vessel in which it is stored (how often cleaned, and whether covered to exclude flies and dust). Milk is sometimes placed for coolness where there is a through draught, and, if the vessel is uncovered, dust is readily blown on its surface.

If all these points are satisfactory and the feedingbottle and teat are clean and in good order, it is probable that the quality of the milk is at fault. This may be due to added chemical preservatives (usually boracic acid) or to bacterial infection. These defects must be rectified by obtaining a different supply of milk, or, alternatively (in the case of bacterial impurity), by boiling or by pasteurisation. ${ }^{1}$ If these measures fail and no form of cow's milk can be tolerated, some

1 Pasteurisation of Milk.-In this method the milk is kept at a temperature of $160^{\circ} \mathrm{F}$. for 20 minutes. The feeding-bottle containing the milk is closed by a cotton-wool plug, or by a special rubber cap, and placed on a tray in a saucepan of water. The saucepan is then covered by a lid through which passes a thermometer so adjusted that its bulb dips into the water. When the milk has been heated the required time, the whole apparatus is allowed to stand unopened for another 20 minutes. The bottle is then removed, the plug withdrawn, and a rubber teat fixed to the neck. 
other food must be tried. This last step should be only temporary and the child should be brought back to its more natural nourishment as soon as possible. In obstinate cases, before replacing the cow's milk by another food, it is often well to remember the beneficial effect of a brief period of complete abstention from food. Though contra-indicated by collapse or exhaustion, a fast of from four to eight or even more hours, is sometimes productive of improvement when feeding is resumed. Longer fasts can be tried, especially if water is given by the mouth or by the rectum.

Of temporary substitutes for milk, trial may be made of condensed milk, ${ }^{1}$ dried milk, ${ }^{2}$ ass's milk, ${ }^{3}$ goat's milk, ${ }^{4}$ peptonised milk, ${ }^{5}$ albumin water, ${ }^{6}$ whey, ${ }^{7}$ sherry-whey, ${ }^{8}$ sugar solution, ${ }^{9}$ or normal saline solution. ${ }^{10}$ If vomiting

${ }_{1}$ Sreetened condensed milk should be diluted with water seven times, and one teaspoonful of cream added to every six tablespoonfuls of the mixture. Unsweetened condensed milk should be diluted four times, with a teaspoonful each of cream and of sugar to every six tablespoonfuls.

${ }^{2}$ Several varieties are in the market.

${ }^{3}$ Ass's milk, containing but little caseinogen, provides a flocculent curd. It contains, however, less than a third the amount of fat in cow's milk, and has the further disadvantage of being laxative.

${ }^{4}$ Goat's milk has a composition not very unlike that of cow's milk, and possesses two advantages over the latter. It is less liable to tuberculous infection and less subject to fæcal contamination, as the droppings of the goat are solid. It has the disadvantage of a strong flavour.

${ }^{5}$ Peptonised milk may be made by heating a pint of milk and five fluid ounces of water to $140^{\circ} \mathrm{F}$., and then adding twenty grains of sodium bicarbonate and one fluid drachm of pancreatic solution (B.P.). The mixture is maintained at a temperature of from $112^{\circ}$ to $140^{\circ} \mathrm{F}$. for twenty minutes to half-an-hour, and is then momentarily heated to boiling-point.

${ }^{6}$ Albumin water is made by adding the white of one egg to half-a-pint of water and mixing thoroughly by shaking. It may be sweetened and flavoured.

7 Whey is prepared by adding a teaspoonful of essence of rennet to half-a-pint of milk warmed to $96^{\circ} \mathrm{F}$. Allow it to set before the fire, then break up the curd, allow it to settle, and pour off the whey.

8 Sherry-whey is made by adding a wineglassful of sherry to half-a-pint of milk just boiling. Heat again to boiling-point and strain through muslin.

${ }^{9}$ Six teaspoonfuls of milk-sugar to a pint of water.

${ }^{10}$ One teaspoonful of common salt to a pint of boiled water. 
is persistent, any, except the first four of these, may be given by the rectum, or the last subcutaneously.

It is the usual practice to treat the continued vomiting of infants, inter alia, by an increased dilution of their feeds, and the results, in most cases, justify the remedy. There is the danger, however, especially in prolonged cases, that the continued starvation may bring the child's vitality still lower. Sometimes these cases improve by exactly contrary measures-namely, by increasing the strength of the feeds or by giving undiluted cow's milk. This last method is particularly useful in these cases of vomiting, as much smaller feeds can be ordered and yet more nourishment can be administered. If sodium citrate is added to the pure milk in the proportion of one to two grains per ounce of milk, a valuable remedy is to hand.

C. Improper Food-stuffs.-The symptoms may result from the use of a food not adapted to the particular age or even unsuited to infancy at all. Enquiry should not stop short at a general question as to whether any food has been given likely to disagree with the patient. Mothers will give their babies the most improper foods without knowing their error, and will therefore reply to a general question in the negative. A more detailed interrogation respecting the common articles in an adult diet will sometimes lead to enlightenment. 


\section{N D E X}

A

Abdomen, distension of, 334

- examination of, $2 \mathrm{~S} 9,303$

- prominence of, in deformity of chest, 303

- resistance of, on palpation, 303

Abdominal pain, referred, 301

- tumours, cardiac impulse and, 312

- effect of, on ribs, 303

Abortion, criminal, 248

Abscess, alveolar, from dental caries, I 42

- retro-pharyngeal, and sudden death, 279

Acardiacus, 7

Accommodation of sight, 123

A.C.E. mixture, effect of, on respiration, 306

Acetanilide, 320

Acetonuria, recurrent vomiting with, 309

Achondroplasia, 8

Acland, Dr, quoted, 104

Acne, rarity of, in childhood, 267

Acquired idiocy, 196, 200

- - traumatic, 20I

- - varieties of, 197

Acts of Parliament, etc., referred to-

Children Act, I908, $25 \mathrm{I}$

Criminal Law Amendment Act, I885, 2 I 2

Dairies, Cowsheds, and Milkshops Order, I 885,96

Education (Administrative Provisions) Act, 1907, I28, 149

- (School Attendance) Amendment Act, I 899, I 33

Elementary Education (Defective and Epileptic Children) Act, I 899, I 88

General Powers (London County Council) Act, I907, 257

Infant Life Protection Act, I897, 250

- - Act, 1909, 249

Notification of Births Act, I907, 247
Acts of Parliament, etc., referred to, contd.-

Provision of Meals Act, IO2

Public Health (London) Act, I89I, I I4

Statute of Prerogatives, I87

Acute illness, children in, 276

- - treatment of, 316

Addison's disease, rarity of, in childhood, 267

Adenitis, cervical. See Glands, ENLARGED

Adenoids. See Tonsils AND Adenoids

Adhesions, amniotic, effects of, 6

Adolescence, training at, I 74

Adolescents, mental characteristics of, I74, I 76

Adrenal glands, fœtal, I 3

- - growth of, $3 \mathrm{I}, 32$

Adrenalin absent before birth, I 3

Esthetics in education, 169

Affection, growth of, 88

Age, bearing of, on diagnosis, 294

- incidence of disease, 265

- - of heart disease, 295

- minimum, for school attendance, 172

Ainy-le-Château, I92

Alre nasi, movements of, 307

Albuminuria in diphtheria, 308

- in new-born infants, 39

- orthostatic, epistaxis in, 3I I

Alcaptonuria, 4

Alcohol, children tolerant of, 320

- medicinal use of, $32 \mathrm{I}$

- mental deficiency and, I94

- schoolboys and, IO3

Alcoholism, maternal, effects of, on child, 8 - rarity of, in childhood, 267

Altherr and Quetelet quoted, 45

Altruism in adolescence, I 74

- in childhood, 88

Amaurotic genetous idiocy, 199

Amnion, 5 
Amniotic adhesions, 6

- fluid in fœtal stomach, I 3

Amphibian eggs, experiments on, 5

Amputation, intra-uterine, 6

Amylopsin, 35

Anæmia, dental caries and, 142

- pernicious, rarity of, in childhood, 267

Anæsthetics, effect of, on respiration, 306

Anencephalics, crying of, 6I

Anencephalus, 6

Aneurisms, rarity of, in childhood, 267 , 305

Animal heat. See HEAT, ANIMAL

Antenatal physiology. See Physiology, ANTENATAL

Anterior poliomyelitis, age-incidence of, 268

Antrum of Highmore, 24

Aortic regurgitation, rarity of, in childhood, 305

Aperients, 334

Apex-beat. See CARDIAC IMPUlse

Apomorphine, 320

Appendicitis simulated by pneumonia, $30 \mathrm{I}$

- rigors in, 3 II

Appetite as an index of health, 276

Arhythmia not common in childhood, 305

Arm-bones of infant, 26

Arms, use of, by infants, $64,65,67,68,74$

- use of, developing ideas of self, 70

Arrowroot-water, recipe for, 332

Arsenic, 320

Art. See ÆSTheTiCs

Arteries, diseases of, rare in childhood, 305

- sectional area of, 38

Arterio-sclerosis rare in childhood, 267

Articulation. See SPEECr

Artificial and natural stupidity, I 37

- feeding of infants. See INFAnT-FEEDING

Ascites, palpation of abdomen in, 303

- effect of, on cardiac impulse, 3 I 2

Asphyxia at birth producing idiocy, 200

Asphyxiation from food in air-passages, 279

Association, mental, 63, 66, 70, 80

- - between objects and sounds, 78

Ass's milk, 335

Astigmatism, I 24

Astonishment. See WONDER

Asylums, idiot, I 88

- - private, 208

Ataxy, congenital, 34

Atelectasis, I 7, 302

- a cause of sudden death, 279

Atheroma rare in childhood, 268

Atmospheric pressure, effect of, on chest, 27
Atmospheric temperature. See TEMPERATURE, ATMOSPHERIC

Atresia of colon, constipation in, 3 Io

Atrophy. See Wasting

Atropine, 320

Attendance, average, and infectious fevers, I I 2 I 32

- minimum age of school, I 72

Attention, power of, 75

- - - in idiots, 202, 204

- - - in school-children, IO4

Authority, place of, in child-training, 167

Automatic movements of infants, $6 \mathrm{I}, 67$

Auscultation, 286, 287, 3I 2

- facilitated by crying, $28 \mathrm{I}$

\section{B}

Baby-farming, 248, 250

Back, shape of infant's, 27

Bacteria, transmission of, to child in utero, 8

Baked flour, recipe for, 326

Baldwin, J. M., quoted, $56,63,66$

Band of Hope classes, spread of infectious diseases by, II 3

Barley-water, recipe for, 332

Barr, Dr, quoted, 209

Basket-weaving for mentally deficients, 2 I 4

Baths, cold, in fever, 324

- hot, 323

Beading of ribs, 284

Bead-threading for mentally deficients, 2 I 4

Beds, folding, in boarding-schools, 85

- size of, in boarding-schools, 95

Bee, incident of child and, 86

Belgium, mentally deficients in, 192

- seaside resorts suitable for children in, 3 I 9

Belladonna, 320

Belt, elastic, effect of wearing, 270

Bicêtre in Paris, 206

Bile, secretion of, before birth, I 3

Birth, injury at. See INJURY AT BIRTH

- mechanical forces acting at, I4

- palsy, idiocy from, 200

- - - from, surgical treatment of, 203

- -rate, England and Wales, 220

Bismuth salicylate, 334

Biting his arm, incident of child, 82

Bladder, contents of, before birth, I3

- loss of control of, 297

Blame, sensitiveness to, I6I

Blepharitis, marginal, I 36 
Blind mentally deficient children, 208

Blindness in primary amaurotic idiocy, I 99

Blinking in infants, 64

Blood, examination of, $29 \mathrm{I}$

- pressure, 38,305

- relative amount of, at different ages, 37

- specific gravity of, at puberty, 55

Boarding-schools-

Advantages of separate "houses," 93

Aspect of buildings, 93

Alcohol in school diet, I03

Closet accommodation, 95

Closure during epidemics, I I6

Diet, 102

Dining-hall, 93

Hygiene, standard of, 92

Infectious diseases, I I 6

Leisure, use of, I 72

Laundries, 96, i 16

Meals, times of, IO2

Medical officers, I 50

Medical supervision, I 49

Milk-supply, 96, I I6

Site, 93

Soil, 93

Studies as bedrooms, 95

Water-supply, 96

Board of Control, I 90

- of Education and medical inspection, I 29

- - - schedule of medical inspection, I 38,140

Body-surface and body-weight, relation between, 36

Body-weight, absolute increase in, 32

- and body-surface, relation between, 36

Bone-disease causing pseudo-paralysis, 289

Boracic acid in milk, 324

Bottle-feeding. See INFANT-FEEDING, ARTIFICIAL

Bottle, hot-water, effect of, on temperature, 323

Bow-legs among public schoolboys, I 49

Boys and girls, comparison between, 54

Brain. See also Cerebral, mental

- compression of, and still-birth, I4

- effect of want of food on, IOI

- growth of, 32, 33

- injury to, causing idiocy, 200, 201

- protrusion of, from head, 6

- rupture of embryonic, 6

- weight of, at different ages, 33

Breast-feeding. See also INFANT-FEEDING, NATURAL

- - bottle-feeding and, 253

- - diarrhœa and, 255

- - duration of, 326
Breast-feeding, infantile defects interfering with, 35

- - insufficient, 332

- mechanism of, 34

- method of, 327

- - rickets and, 253

- - tubercle and, 258

Breasts, care of, in pregnancy, 327

- wet nurse's, 328

Bridgman, Laura, 202

Bright's disease, epistaxis in, 3 I I

- - primary chronic, rare in childhood, 267

Bromides, effect of, on developing toad, 5

- per rectum, 325

- tolerance of, by children, 320

Bronchitis, acute, effect of, on respiration, 307

- - relative frequency of, 296

- - shape of chest altered by, 302

- - stridor in, 308

Bronchophony, how to examine for, 28I

Broncho-pneumonia, effect of, on respiration, 307

- infant mortality from, 230, 232

- relative frequency of, 296

Bruits, diagnosis between congenital and acquired, 295

- subclavicular, 3 I 2

Brushwork for mentally deficients, 214

Brussels, myopia in schools in, I 24

Butter a vehicle for powders, 322

\section{C}

Cachets, 321

Callousness, 88

Calomel, 320, 334

Calorie, 48

Calorific value of cow's milk, 5 I

- - of food required by infant, 49

- - of human milk, 49

Camerer quoted, 44, 53

Camphor, 320

- compound tincture of, dose of, $32 \mathrm{I}$

Canadian farm-colonies for mentally deficients, I 92

Cancer, rarity of, in childhood, 267

Cantines scolaires, 102

Capsules, $32 \mathrm{I}$

Carbohydrates, consumption of, 44

- in school-dietary, IO3 $\mathrm{V} \exists \mathrm{g}_{\mathrm{M}}$

Carbon dioxide, excretion of, 39

- — in class-room air, 98, 105 
Cardamoms, compound tincture of, 321 Cardiac impulse, displacement of, 303

- - variations in position of, $3 \mathrm{I} 2$

Care-takers, phthisical, I 20

Caries, dental. See DenTAL C.ARIES

Carpentry for mentally deficients, 2 I 4

Castor oil, 334

Cataract, medical inspection for, I 36

Catechu, 334

Catheter, size of, for gastric lavage, 325

Catheterisation, 290

Cats, heat-regulation in new-born, 43

Cerebellum, function of, 33

- growth of, 33

Cerebral degeneration, infantile, I 99

- disease, effect of, on respiration, 307

- embolism causing idiocy, 201

- sinus thrombosis, epistaxis in, 3 I I

- - - rigors in, 3II

- symptoms in infants, 277,297

- - in pneumonia, 277

- thrombosis causing idiocy, 20I

- tumour, convulsions in, 277

- - moral insanity from, 216

- - vomiting in, 309

Cerebro-spinal dropsy of embryo, 6

- - meningitis. See MENINGITIS

Cerebrum. See also BRAIN

- inactive before birth, I2

Chalk in gastro-intestinal disease, 334

Chamberlain, A. F., quoted, 245

Charlottenburg open-air school, I 48

Chemical composition of infants and adults, 34

Chest. See Thorax

Chewing the cud, 4

Cheyne-Stokes breathing, 38

Chick, experimental deformity of, 5

Chicken-pox, infant mortality from, 230

- loss of appetite after, 276

- wasting after, 275

Child-rearing, instruction in, I81, 259, 264

Children, abnormal types of, I 83

- Act, I 908, 25I

- antipathy of, to disease, 272

- peculiarities of, in disease, 27I, 276

Chloral, 320

- per rectum, 325

Chlorides, effect of, on developing toad, 5

Chloroform, effect of, on respiration, 306

Chlorosis, rarity of, in childhood, 267

Chocolate creams, powders administered in, 322

Chorea, age-incidence of, 269

- medical inspection for, 136

- relative frequency of, 297

Circulation, changes in, at birth, I 7
Circulation, respiration and, 36

- time, 37

Cirrhosis of liver, rarity of, in childhood, 267

Citrated cow's milk, 336

Clasping, reflex act of, 6 r, 64

Class-rooms, 98-

Acoustic properties, 98

Desks, I0O

- harmful effects of, I 22

Dirt and phthisis, i 8

Floor, danger from, I I4, I 8

- space, 98

Furniture, 99

Heating, 98

Illumination, 99

Size, 98

Temperature, 99

Time-table and mental fatigue, I05, 106

Ventilation, 98, 105

- effects of bad, I22

Wall-colouring, 99

Clavicle in infants, 27

Clay-modelling for mentally deficients, 2 I 4

Cleft palate, effect of, on suckling, 35

- - examination for, 292

Climbing stairs, 83

Closets in boarding-schools, 95,98

- in elementary schools, 97

Closure of boarding-schools for infectious outbreaks, i I 6

- of elementary schools for infectious outbreaks, I I4

Clothing, children's, Io9

Clubbed feet and idiocy, 205

- fingers, 285, 303

Codeine, 320

Co-education, 178

Cold, sense of, $57,60,62$

Collapse, hot baths in, 323

- rectal injections in, 324

Colitis, treatment of, by rectal lavage, 325

Colon, diseases of, causing constipation, 310

Colour sense, 64,87

- - in mentally defectives, training of, 2 I 3

Coma in pneumonia, 277

- nasal feeding in, 325

Combined defects, 208

Comenius, I 56

Comforters, rubber, risks of, 256

Committee, Departmental, of Home Office, on half-timers, I 34

- for the care of the feeble-minded, I 90

Compensatory breathing, 313 
Complications, frequency of, in children, 278

Composition, chemical, of infants and adults, 34

Compound liquorice powder, 322

- tincture of camphor, dose of, 321

- - of cardamoms, 32 I

- - of lavender, 321

Condensed milk. See Milk, CONDENSED

Congenital ataxy, 34

- deformities. See Defects, conGENITAL

- idiocy, 196

- laryngeal stridor, 308

- stenosis of colon, 310

- - of pylorus, gastric lavage for, 325

Congress, International, on School Hygiene, 128

Conjunctivitis, medical inspection and, I36

Consanguinity as a cause of mental deficiency, 194

Consciousness of self. See SElf, CONSCIOUSNESS OF

Consonants, use of, by infant, $66,77,78$

Constipation, causes of, 3 Io

- diagnostic value of, 309

- febrile attacks from, 3 Io

Consumption. See PHTHIsis, Tubercle

Contagious skin diseases in schools, I 20

Convulsions, acute diseases and, 277

- as a cause of death, 226,232

- diseases characterised by, 232

- idiots and, 200

- infant mortality from, 228, 229, 234, 235

- - - in Evelina Hospital, from, 234, 235

-23 of legitimate and illegitimate infants from, 226

- treatment of, by hot baths, 323

- use of term in death-certification, 237

Cookery, training of girls in, 81

- - of mentally deficients in, $2 I_{4}$

Co-ordination of muscles, $61,65-$

Arms, 68, 78

Eyes, 64

Head, 64

Leg, 76

Trunk, 68

Co-ordination, defective, of head, a sign of idiocy, 204, 302

- loss of, in amaurotic idiocy, 199

- mentally defectives and, 2 I 4

- training of, in infants, 165

- - of, in mentally deficients, 214

Cord, spinal. See SPINAL CORD
Corneal opacities, medical inspection and, I 36

Cough, varieties of, 284

Coughing, reflex act of, $6 \mathrm{r}$

Counter-irritation, 323

Court of Wards and Liveries, 187

Crawl, first attempts to, 76

Crêches, 25I, 263

Cretins, characteristics of, 198

- hearing of, 202

- treatment of, 203

Crichton-Brown, Sir James, 106

Crime, mentally deficients and, 192, 212

Criminal Law Amendment Act, I885, 2 I 2

Crippled mentally deficient children, 208

Crowing, 69, 78

Crying in infants, $61,63,68$

- - auscultation facilitated by, 28I

- - - pain as a cause of, 300

- - - speech originating in, 78

- - - varieties of, in disease, 284

Cubicles in boarding-schools, 94

Curiosity an influence in child-training, I 60

$\mathrm{D}$

Dairies, Cowsheds, and Milkshops Order, I 885,96

Darenth Idiot Asylum, 188

Darwin quoted, $56,63,76,199$

Day nurseries, 263

- National Society of, 263

- schools, 97

Deaf-mute mentally deficients, 208

Deafness, congenital, of cretins, 202

Death-rate, England and Wales, 220

- infant. See InfANT MORTALITY

Death, sudden, of infants, 279

Debility. See also WAsting

- treatment of, 318

Deductive reasoning in children, 175

Defæcation, frequency of, in infants, 36

- mechanism of, in infants, 36

Defects, congenital, 4,6

- - causes of, 243

- - infant mortality from, 334, 335

- - infant mortality, legitimate and illegitimate, from, 226

- - when produced, 243

Deformity, experimental, of amphibians, 5

- - of chick, 5

Degeneration, physical, as a cause of infant deaths, 242,243

- - report on, 244 
Deglutition before birth, I 3

Délapine, Dr, quoted, 257

Delirium in pneumonia, 277

Dental caries, cause of, 142

- - dyspepsia from, I42

- - effects of, I4I

- epilepsy from, $\mathrm{I}_{42}$

- - examination for, 292

- - in Evelina Hospital, I4 I

- - in school-children, I4 I

- - tubercle from, I42

- surgeons for schools, I 43

Dentition, delayed, 205, 302

Deprivation, idiocy by, $20 \mathrm{I}$

Diabetes insipidus, 4

Diagnosis of idiocy in infants, 204

— of hydrocephalic and rachitic heads, I 98 , 302

Diagnostic points in connection with-

Abdomen, 303

Age, 294

Appendicitis, 301

Ascites, 303

Atelectasis, 302

Auscultation, 3 I 2

Bronchial obstruction, 302

Cardiac impulse, 303

Cerebral disease, 297

Clubbed fingers, 303

Common acute diseases, 296

- chronic diseases, 296

Constipation, 309

Crying, 300

Development, irregular, 302

- delayed, 302

Diarrhcea, 3 IO

Empyema, 302

Epidenics, 295

Epistaxis, 3 I I

Facial expression, 300

Fibroid lung, 302, 3 I 4

Flatulence, 303

Fontanelle, 30I

General appearance, 297

Head, shape of, 302

Heart-disease, 295, 302, 3 I I

Hip-joint disease, $3 \circ \mathrm{I}$

Hysteria, 294

Intussusception, 299, 3 Io

Meningitis, 299

Murmurs, 295

Neurosis, 295

Otitis, 300

Pain, 300

- referred, 30 I

Pericarditis, $30 \mathrm{I}$

Peritonitis, tuberculous, 303
Diagnostic points in connection with, contd.Pleural effusions, 3 I 2,3 I 3

Pneumonia, 299, 30I, 3 I 2

Pulse, 305

Respiration, 306

Rigors, 3I I

Seasons, 295

Sex, 294

Spinal caries, $30 \mathrm{I}$

Stridor, 308

Temperature, 304

Thorax, deformities of, 302

Tonsils, 303

Vomiting, 309

Diaphragm, paralysis of, 307

- restricted action of, 307

Diarrhœa, causes of, 3 I0, 33 I, 333

- convulsions in, 277

- cow's milk and, 253

- diagnostic value of, 3 IO

- epidemic, 253

- - age incidence of, 254, 255

- - atmospheric temperature and, 254

- - in Evelina Hospital, 255

- - hand-feeding and, 255

- - infant mortality, urban and rural, from, 255

- - insanitation and, 254

- - preventive measures, 256

- infant mortality from, 228, 229, 234, 235,236 $-\frac{2}{226}$ legitimate and illegitimate, from,

- - - urban and rural, 255

- relative frequency of, 296

- treatment of, 334,335

Diet. See also INFANT-FEEDING

- children's, 53

- infant's, 45,326

- - in disease, $33 \mathrm{I}$

- school, IOI

Digestion, organs of, before birth, I2

- physiology of, 34

Dilatation of colon causing constipation, 3 IO

- of heart, 267

Diluents of milk, 332

Dining-halls, school, 93

Diphtheria, albuminuria in, 308

- epidemics of, in schools, I I6

- glands enlarged in, 144

- infant mortality from, 230

- laryngeal, altering shape of chest, 302

- - stridor in, 308

- moral insanity from, 2 I 6

- paralysis of diaphragm in, 307 
Diphtheria, seasonal prevalence of, 295

- spread of, by schools, II 2

Diplegic idiots, 200

"Dirt-conditions," 120

Dirt, influence of, in infant life, 252

Disappointment, expression of, by infants, 69

Disciplinary training, 167

Discrimination, power of, in infants, 66

Disease, convalescence from, 278, 317

- course of acute, 278

- incidence of, in childhood, 265

- - of, factors diminishing, 266

- - of, factors increasing, 268

- mental attitude of children towards, 272

- muscular activity in, 273

- peculiarities of children in, 271, 276

- simulated, 273

Diseases, common acute, 296

- - chronic, 296

- relative frequency of, 295

Distance, appreciation of, by infants, 68 , 74

Dogs, heat-production in, 42

- heat-regulation in new-born, 43

Domestic economy, instruction of girls in, I $8 \mathrm{I}$

Donders quoted, 6o

Dormitories, 94

Dorsetshire, infant mortality in, 222

Dose of Dover's powder, $32 \mathrm{I}$

- of opium, 320, $32 \mathrm{I}$

Doses, formula for calculating, 320

- suited to children, 3 I9

Dover's powder, 321

Drainage in boarding-schools, II 6

- in elementary schools, 97

Drawing, 89, I 06

- training mentally deficients in, $2 \mathrm{I}_{4}$

Dress, children's, 109

Dressmaking, training of girls in, 18 I

Dropsy, cerebro-spinal, of embryo, 6

Drowsiness in pneumonia, 277

Drugs, reaction of children to, 320

Drug-taking, rarity of, in childhood, 267

Duchenne's muscular atrophy, 270

Ductless glands in fœtus, I3

Ductus arteriosus, closing of, $\mathrm{I} 7$

Dukes, Dr, quoted, 92, I06

Dun-sur-Auron, mentally deficients at, 192

Durham, infant mortality in, 223

Dust, protection of milk from, 256

Dyspepsia from dental caries, $\mathbf{I}_{42}$
E

Earlswood Idiot Asylum, epileptics in, 201

Ears, medical inspection of, 136

- shape of, in idiots, 205

Eau de Cologne, application of, to nipples, 327

École alsatienne, $\mathrm{I} 68$

Education (Administrative Provisions) Act, I 28, 149

- authorities, local, duties of, re medical inspection, 128

- (School Attendance) Amendment Act, 133

Egg, experiments on developing, 5

Egoism in children, 88

Eider-down, incident of child with, 87

Elementary Education (Defective and Epileptic Children) Act, 188

Elephant, pulse-rate of, 37

Embolism, cerebral, a cause of idiocy, 201

Embryo, diseases of, 6

- protection of, 6

- rate of growth of, 245

Emotional idiocy, 197

Emotions. See also Feelings

- development of, $59,60,62,65,69,75$, 78,87

- of idiots, 203

- morality and, 88

- short-lived in children, 88

Emphysema, rarity of, in childhood, 267

Employment of children, I 33

- of married women, infant mortality and, $246,251,262$

Empyema altering shape of chest, 302

- diarrhœa from, 310

- rigors in, 311

Encephalitis a cause of idiocy, 201

Endarteritis, rarity' of, in childhood, 267

Endocarditis, 295

Enema rash, 325

Enemata, aperient, 325

- medicinal, 325

- nutrient, 324, 336

- saline, 325

Energy, amount of, required for growth, $4 \mathrm{I}$

- consumption of, from food, $4 \mathrm{I}$

- loss of, in illness, 273

Enteric fever. See TYPHOID FEVER

Enteritis, tuberculous, diagnosis of, 3 I I

Entero-colitis, relative frequency of, 296

- - treatment of, by rectal lavage, 325

Enthusiasm in childhood, 160 
Enuresis, 269

- mouth breathing and, 143

Environment, disease and, 266

- intra-uterine, 5, 6, Io, I I

Epicanthus in idiocy, 205

Epidemic, diarrhœa. See DIARrhœA, EPIDEMIC

Epidemics, prevalent, in relation to diagnosis, 295

- in schools, 116

Epileptic colony at Ewell, 209

— idiocy, 200

- - treatment of, 203

- mentally deficient children, 208

Epileptiform convulsions. See ConvulSIONS

Epilepsy, dental caries and, I42

- mental deficiency and, I42

- moral insanity and, 216

Epiphyses, disease of, 269

- examination of, 285

- pseudo-paralysis from disease of, 289

Epiphysitis, syphilitic, 269

Epistaxis, causes of, 3 I I

Erect posture, effect of, on height, 29

Erythema nodosum, 269

Ethical training of children, I68, I74

Eugenics, 5, I 82

Evelina Hospital, dental caries at the, I4I

- - infant mortality at, 233

- - summer diarrhœa at, 255

Evening preparation, I06

Evolution, relation of, to education, I 56 , 162

Ewell Epileptic Colony, 209

Examination, clinical, of children-

Abdomen, 284, 289, 303

Auscultation, 286, 287

Beaded ribs, 284

Blood, 29I

Chest, shape of, 284

Clubbed fingers, 285

Cough, 284

Crying, 28I, 284

Epiphyses, 285, 287

Fæces, 29I

Fontanelle, 284

Harrison's sulcus, 284

Head, shape of, 284

History of case, 283

Inspection, 284

Nervous system, 289

Pain, 285, 287

Palpation, 286

Percussion, 289

Position of patient, 281, 282, 285, 289, 290
Examination, clinical, of children, contd.-

Pulse, 29I

Rectum, 290

Respiration, 284

Sputum, 29I

Temperature, $29 \mathrm{I}$

Throat, 292

Urine, 290

Vomit, 291

Examinations, school, I 57, 175

Excess, habits of, rare in childhood, 267

Exophthalmic goitre rare in childhood, 267

Expectoration. See SPUTUM

Expression of pain by infants, 300

- power of, in infants, $60,62,65,69,75$, 78

Eyebrows, movements of, indicative of pleasure, 75

Eyelids, movements of, 61, 62, 64

Eyes, medical inspection of, I 36

- movements of, 6I, 64

Eyesight. See SIGHT

\section{F}

Face, expression of, in mouth-breathers, I 43

- small size of, in infants, 24

Facial expression of feelings, 65, 69, 75

Fæces, characteristics of infantile, 36

- examination of, $29 \mathrm{I}$

- incontinence of, 290,297

Family-care of mentally deficients, 192

Farm-colonies for mentally deficients, 192

Fat, amount of, in body, 34

- consumption of, by infants and adults, 44

- deposit of, in girls at puberty, 55

- proportion of, retained for growth, 44

- school dietary and, IO3

Father, health of, as affecting unborn child, 9

Fatigue, classroom time-table and, 105, I06

- schoolwork and, 104

Fauces, examination of, 292

Favus, I 20

- school, I 2 I

Fear, development of, in infants, 66,87

- its diagnostic implication, 299

Feather, incident of child and, 87

- name of, applied to pigeon, 84

Feeble-minded. See MeNTALly DEFICIENT 
Feeding-bottles, 328

Feeding, infant. See INFANT-FEEDING

Feeds, infant. See INFANT-FEEDING

Feelings. See also Emotions

- expression of, by infant, $60,62,65$, 69

- pleasant, how produced, 62, 65

- unpleasant, how produced, 65

Femur at birth, 25

- changes in, 26

Fermentation, gastric, 333

Fever, reduction of, by rectal injections, 324

Fevers, specific, convulsions in, 277

- - idiocy following, 201, 202

- - infant mortality from, 228, 229, 230

- - intra-uterine, 8

- - moral insanity from, 216

- - relative frequency of, 296

- - schools and. See SCHOols, INFECTIOUS FEVERS

\section{- - vomiting in, 309}

Fibroid lung altering state of chest, 302

- - diagnosis of, from pleurisy, $3 \mathrm{I}_{4}$

- - effect of, on cardiac impulse, 312

Fingers, clubbed, 285, 303

- infant's interest in his, 67,80

- webbed, 4

Fire-places in classrooms, 98

Fissure, rectal, causing constipation, 3 Io

Flat-foot among public schoolboys, 149

Flatulence, 331, 333

- abdominal palpation in, 303

- effect of, on cardiac impulse, 3 I 2

Flies, infection of milk by, 256, 334

Floors, dangers of classroom, I 4

- - of nursery, 165

Floor-space in elementary schools, 98

Flour, baked, recipe for, 326

Focal length of eye, 123

Fœtus, physiology of, at term, I I

- rate of growth of, 245

Fomentations, 323

Fontanelle, closing, date of, 30I

- diagnostic importance of, 299, 30r

- examination of, $284,29 \mathrm{I}$

- hydrocephalic idiot's, 197

Food. See also INFANT-FEEDING

- heat produced from, 43

- relation between growth and, $4 \mathrm{I}$

- requirements of infant, 43

- - _ - estimate of, 46

- school, IOI, IO2

- want of, effect on mental work, Ior, I 33

Foods, proprietary. See Proprietary FOoDS
Foramen ovale, closing of, I7

Forehead of genetous idiots, 200

- of microcephalic idiots, 197

Foreign bodies, stridor from, 308

Formula for calculating doses, 320

- - - surface-area, 46

Fractured skull, idiocy from, 20I

France, medical inspection in, I 27

- mentally deficients in, 192

- seaside resorts for children in, 319

Frequency, relative of common diseases, 295

Friedreich's ataxy, 270

Frontal sinus in infants, 25

Fundus of eye, examination of, 290

Furniture, classroom, 99

\section{G}

Galabin, Dr, quoted, 245

Galton, Mr Douglas, 125

Games, I08

- compulsory, I09

Gamgee-tissue jackets, 323

Gardening, training of mentally deficients in, 214

Gastric juice in infants, 35

- lavage, 325, 334

- ulcer, rarity of, in childhood, 267

Gastro-enteritis, convulsions in, 277

- lavage for, 325, 334

- relative frequency of, 296

Gavage, 325

Genetous idiocy, 200

Germany, seaside resorts for children in, 319

Gesticulation, expression of feelings by, 65 , 69

Gheel, mentally deficients at, I 92

Gingivitis, dental caries and, I 42

Girls, co-education of, 178

- educational differences between boys and, 177

- influence of teachers on, I82

- instruction of, in child-rearing, 181

- - of, in domestic economy, I8I

- of, in healthy living, I79

- physiological differences between boys and, 54

- training of, 177

Glands, ductless, in fœtus, I 3

- enlarged, altering shape of chest, 302

- causes of, I44

- - dental caries and, I42 
Glands, enlarged, frequency of, I44

- - lymphoid, 40

- - measles as a cause of, 144

- - medical inspection for, I 37

- pediculosis and, I44

- - stridor from, 309

- - sudden death from, 279

Goat's milk, 335

Goitre in new-born dogs, I3

- - - infants, I 3

- exophthalmic, rarity of, in childhood, 267

Goodhart and Still quoted, 50, 258

Gout, rarity of, in childhood, 267

Gouttes de lait, 26I

Government grants and spread of infectious fevers, II 2

Grasping movements before birth, I3

- - after birth, $64,65,67,68,74$

- - defective, as a sign of idiocy, 204

Gravity, effect of, on intra-uterine development, 5,23

- ${ }^{2}$ of, on skeleton, 25

Growth, food and, 4I, 44, 276

- in height, 28

- impaired in disease, 269,274

- - by mitral stenosis, 275

- irregular, diagnostic value of, 302

- of organs, 3 I

- physiology of, 2 I

- rate of, in utero, 245

- underfeeding and, 45

- in weight, 29

Grunting, 78

Guardians of the poor, mentally deficients and, 188

Guardianship of mentally deficients, I92, 207

Guinea-pigs, heat-regulation in new-born, 43

- respiratory centre of, 38

\section{$\mathrm{H}$}

Habits, formation of, I 59

Habit, cultivation of, in infants, 166

- -spasms, I 42,274

Hæmatoxylin in gastro-intestinal disease, 334

Hæmoglobin destroyed by foctal liver, I 3

Hæmophilia, 4

- epistaxis in, 3 II
Hæmorrhage, cerebral, idiocy from, I5, 200

Half-timers, 133

Haller quoted, I 94

Hand-feeding. See INFANT-FEEDING, ARTIFICIAL

$\mathrm{H}$ ands, use of, by infant, 67

Hare-lip, effect of, on suckling, 35

Harrison's sulcus, 284

Head, aversion of, as a sign of refusal, 66

- hydrocephalic, 197, 302

- operations on, 203

- injury causing moral insanity, 2I 6

- microcephalic, 197

- - operations on, 203

- movements in infants, 64

- - defective, as a sign of idiocy, 204, 302

- proportions of, in infants, 24

- rachitic, I98, 302

- relative size of, in infants and adults, 24

- retraction, 299

Headache from bad classroom ventilation, I 22

- - dental caries, I42

- - eye-strain, I 23

- - overwork, I25

- - tonsils and adenoids, I37, I44

Head-lice, I 20, I 44

Headlights in classrooms, 99

Health certificates in schools, I I 3

- resorts for children, 3 I9

- Society, Westminster, 260

- visitors, 260

Hearing of idiots, 202

- - training of, $2 \mathrm{I} 3$

- of infants, $57,60,62,65,69$

- testing acuteness of, 136

Heart-beat, age-variations in, 37

- before birth, I 2

- effect of sleep on, 306

- elephant's, 37

- irregularity of, in children, 306

- mouse's, 37

- rabbit's, 37

Heart-disease, congenital, duration of life in, 268

- - in idiots, 205

- diagnosis of, 294, 3 I I

- effect of, on chest, 302

- peculiarities of children in, 275

Heart, growth in weight of, 32

- dilatation of, 267

- displacement of, 303

- in twin conceptions, 7 
Heart, protrusion of, from body, 6

Heat, animal, physiology of, 40

- -loss in adults, 42

- - loss in infants, 48

- - surface-area and, 37,42

- -production, age-variations in, 42

- - in dogs, 42

- - food and, 44

- - pulse-rate and, 37

- - surface-area and, 42

- -regulation, 42

- - in infants, 42,43

- - in new-born animals, 42

- sense of, $57,60,62$

Heel, shape of infant's, 26

Height, growth in, 28

- variations of, 29

Hemiplegia, idiocy from, 200, 270

Herbart, I 56

Hereditary diseases, 267

Heredity, idiocy and, 94

- immaturity and, 246

- moral insanity and, 216

- physical defects and, 4

Herefordshire, infant mortality in, 222

Hernia, idiocy and, 205

- medical inspection of, 140

Holidays, I07, I 7 I

- effect of, on growth, 29

- - of, on specific fevers, I I 2

Holland, seaside resorts for children in, 319

Home and school, moral influences of, I 70

- - - relations between, I08, I I I, I 70, I 72

-work, 106, I07

Homerton, home for mentally deficients at, 209

Hours of school-work, Io6

Housewifery, instruction of girls in, I8 I

- of mentally deficients in, 2 I 4

Howe, Dr, and Laura Bridgman, 202

Hunger, effect of, on infants, 52

- expression of, by infants, $60,62,69$

- influence of, in forming ideas of self, 70

Hydrocephalic idiots, 197

- and rachitic heads, I 98,302

Hydrocephalus, fontanelle in, I97, 301

- internus, 197

- surgical treatment of, 203

Hydrochloric acid in gastric juice of infants, 35

Hygiene of Schools, 92

- of school-life, IOI

- of school classrooms, 98, I2 I
Hypermetropia in school-children, I 24

Hysteria, 272

- diagnosis of, 294

Ice-bags, 323

Ideas, 58

Idiocy, classification of, I 96

- congenital heart-disease and, 205

- diagnosis of, I97, 205

- - of, in infants, 204

- injury at birth and, I5

- measles and, 270

- post-influenzal, 270

- prognosis of, 203

- stigmata of, 205

- treatment of, 203, 2 I I

Idiot asylums, I 88

- - private, 208

- - term defined, I95

- etymology of word, 206

Idiots. See also ImBECILES, Mentally DEFICIENT CHILDREN

- general remarks on, 202

- historical account of, 187

- relative proportions of boys and girls, I 94

- supervision of, 207

- training of, 206, 2IO, 2 I I

Idiots, varieties of-

Birth-palsy, 200

By deprivation, 201

Cretin, I98

Epileptic, 200

Genetous, 200

Hydrocephalic, I97

Microcephalic, 197

Mongolian, I 98

Post-febrile, 20I, 270

Primary amaurotic, 199

Talmuc, Ig8

Traumatic, 201

Iliac fossæ, 25,26

Illegitimacy, 224

Illegitimate children of mentally deficient women, 2 I 2

- - infant mortality of, at successive ages, 225,227

- _ - - of, from certain causes, 226

- - - of, in London, 225, 227

- - - - of, in rural counties, 225

Illumination, classroom, 99 
Image, recognition of, by infant, 7 I, 82

Imagination, 86, 89

Imbeciles. See also Idiots, Mentally DEFICIENT CHILDREN

- moral, i96, 2 Io

- term defined, I95

- training of, 210,2 I I

Imitative movements, $70,75,78$

Immaturity, meaning of term, 244

- mortality from, 244

Immunity, II, 40, 268

Impetigo, I 20, 269

- enlarged glands from, I44

Impulsive movements. See Instinctive MOVEMENTS

Inattention as a sign of ill-health, I25, 274

Incendiarism, mentally deficient children and, 203

Incidence of disease in childhood, 265

Incontinence, 269

- mouth-breathing and, I44

Inductive reasoning in children, 175

Industrial training of children, 89, I 73

- - of mentaliy deficients, 210,212 , 2 I 4

Inebriate mentally deficients, I93, I 96

Inexperience, children's, 85

Infancy, common diseases of, 296

Infant, age to which word is limited, 219

INFANT-FEEDING, physiological principles of, 43

Artificial, 328-

Common errors in, 329

Feeds, composition of, 326, 329

- frequency of, 52,329

- indigestible, 253, 332

- insufficient, 332

- number of, 53, 329

- size of, 53,329

- temperature of, 334

Diarrhœa, 255

Malnutrition, 35, 253, 33I

Milk, cow's-

Amount required, 5I, 52

Caloric value, $5 \mathrm{I}$

Composition, 5 I

Citrated, 336

Diluents, 332

Pasteurised, 334

Peptonised, 335

Preservation, 256, 334

Substitutes, 335

Morbid results, 253, 255, 257, 33 I

Proprietary foods, 330

Tubercle, 257
INFANT-FEEDING (continued)-

Natural (breast), 326 -

Feeds, frequency of, 52

- number of, 53

- size of, 53,328

Diarrhœa, 255

Malnutrition, 35, 253, 33 I

Milk, human-

Amount required, 50, 52

Caloric value, 49

Composition, 49

Deficiency, 253, 332

Rickets, 253

Secretion, 50, 327

Suckling, 34, 327

Weaning, 326, 329

Wet-nursing, 327

Infant foods. See PROPRIETARY FOODS

INFANT LIFE, conditions of-

At birth, 24I

Baby-farming, 248, $25^{\circ}$

Breast-feeding, 253

Children Act, $25 \mathrm{I}$

Congenital defects, 243

Crêches, 25I, 263

Day-nurseries, 263

Degeneration, physical, 242

Diarrhœa, 253

Dirt, 252, 254

Drunkenness, 250

Employment of married women, 246, $25 \mathrm{I}, 262$

Feeding, improper, 253

Hand-feeding, 255

Health societies, 260

Immaturity, 244

Infant Life Protection Acts, 249, 250

Injury at birth, 242

Life assurance, 250

Midwives, 247

Milk, contaminated, 253, 256, 257

- depôts, $26 \mathrm{I}$

Neglect, 250

Notification of Births Act, 247

Post-mortems, 247, $25^{8}$

Poverty, 250

Tubercle, 56

Infant Life Protection Act, I 897, 250

- - - - I gog, 249

INFANT MORTALITY-

Age-factor, 2 I 8

Causes of, 228, 229

Deaths up to 5 years, 2 I 9

Employment of married women, 246

Evelina Hospital statistics, 233

Illegitimacy, 224

Mortality, I85I-I907, 219 
INFANT MORTALITY (continued)-

Mortality, I 900-I 906, 200

- in London, 227

- in Peabody Buildings, 224

- in rural counties, 222,227

- in urban counties, 222

Remedies, 258

Infant protection visitors, 25 I

Infantile cerebral degeneration, I 99

- paralysis, age-incidence of, 268

- scurvy, 269

Infection of child in utero, 8

Infectious fevers. See FEVERS, SPECIFIC

- - in schools. See Schools, INFECTIOUS FEVERS

Influenza causing idiocy, 270

Inhibitory control in infants, 276

Injections, rectal, 324

- subcutaneous, 326, 336

Injury at birth, causes of, 242

- - - idiocy from, I 5,200

- - - infant mortality, legitimate and illegitimate from, $\mathbf{2 2 6}$

- to head causing moral insanity, 216

Insanity, moral, 2 I 5

Instinctive movements, $61,63,66,67,74$, 76

Instincts, animal, of children, I 58

Intelligence, development of, $66,85,89$

- at nine months, 75

- at one year, 80

- training of, $173,175,178$

- - of, in mentally deficients, 215

Internal secretions, 13,55

Intestinal obstruction, 3 IO

Intestines, physiology of, 36

- protrusion of, from body, 6

Intra-abdominal pressure, effect of, on circulation, I7

Intra-thoracic pressure, before birth, 12

- - after birth, I6

- effects of, on chest, 27

Intra-uterine environment, 5, 6, 10, 243

Intussusception, diagnosis of, 297, 310

- vomiting in, 309

Invalid schools. See Schools, SPECIAL

Inverted respiration, 307

Iodides, 320

Ipecacuanha, 320

Ireland, Dr, quoted, 200

Isolation in boarding-schools, I $50,15 \mathrm{I}$

\section{$\mathrm{J}$}

Jaborandi, 320

Jackets, woollen, 223
Jaw, abnormal development of, 270

- effects of growth of, 2 I

- in infancy, 24

Jews, primary amaurotic idiocy among, 199

Joints, use of, by children, 83

Judgments, 58

- erroneous, 86

Jumping, 87

Kaffir babies, feeding with sour milk, 80

- child, incident of, 66

Kerr, Dr James, 98, I 28, I 44

Kidd, D., quoted, 66, 80

Kidneys, cystic, I 3

- fotal, activity of, 13

- growth of, 32

- physiology of, 38

- size of, relative to adrenals, 32

Kilogramme, equivalent of, in ounces, 46

King's protection of natural fools, 187

Kittens, heat-regulation in, 43

Knee-joint, referred pain in, 301

Knock-knee among public schoolboys, I 49

Koch, Prof., quoted, 257

Koplik's spots, examination for, 292

L

Labials, first use of, 78

Lachrymal glands, in crying, 63

Lactation. See BREAST-FEEDING

Lactic acid in infant's gastric juice, 35

Lactose, action of succus entericus on, 36

Lalling, I $45, \mathrm{I}_{47}$

Lambs, incident of child and, 83

Lancashire, infant mortality in, 223

Landois quoted, 38

Langlois quoted, 42

Laryngeal diphtheria. See Diphtheria

- growths, 309

- stridor, congenital, 308

Laryngismus stridulus, 308

Laryngitis, chronic, stridor from, 309

- infant mortality from, 230

Lateral curvature of spine, causes of, I 45

- of spine, in school-children, I22, I44, 149 
Laudanum, dose of, $32 \mathrm{I}$

Laughing (see also SMILING), 65, 69

Laundries, school, 96

Laundry-work, training girls in, I8 $r$

- - mentally deficients in, 214

Lavage, gastric, 325,334

- - to obtain sputum, $29 \mathrm{I}$

- rectal, 325, 334

Lavatories in boarding-schools, 95,98

— in day-schools, 97

Lavender, compound tincture of, 32 I

Lead-poisoning, fœtal death from, 8

- paternal, 9

Legitimacy. See Illegitimacy

Legs, a factor in developing ideas of self, 70

- relative lengths of, in infants and adults, 24

Leisure, use and abuse of, $\mathrm{r} 7 \mathrm{I}, \mathrm{I} 76$

Length, growth in, 28

- infant's, at birth, 28

- relative, of intestines in infants and adults, 36

Lessons, duration of, 105

- home, I07

Lice, head, I2O, I44

Lichen urticatus, 269

Life assurance, infant, $25^{\circ}$

Light, arrangement of, in classrooms, 99

- effect of, on new-born infants, 60, 62

Limbs, influence of, in developing ideas of self, 70

- use of, by infants (see also GRASPING), $6 \mathrm{I}, 63,67,77,78,82,87$

Lime-water, recipe for, 332

Lion, respiration rate of, 38

Lips, sensitiveness of, in infants, 60,67

- pursing the, 75

Liquorice powder, how to administer, 322

Lisping, medical inspection and, 137

Lissauer quoted, 47

Literature, influence of, in child-training, I 70

Liver, activity of fœtal, $12, \mathrm{r}_{3}$

- displaced in deformity of chest, 303

- growth of, 32

Local Government Board, memorandum of, II 5

- - - for Scotland, memorandum of, II 5

Localisation of objects by infant, 68

Locomotion, attempts at, 76,82

- psychological effects of, 60,62

London County Council General Powers Act, I907, 257

Lord Chancellor's control of idiots, I87
Lungs, changes in, at birth, I6, I 7

- diseases of. See also PNEUMONIA, BRONCHO - PNEUMONIA, EMPYEMA,

FYBROID LUNG

- - of, effect on cardiac impulse, 3 I 2

- - of, effect on chest, 302

- - of, effect on respiration, 307

- growth of, 32

一 inactivity of fœtal, I 2

Lying, 88, I 84

Lymphoid tissue, physiology of, 39

- - See also GIANDS, ENLARGED

\section{M}

Macrami-work for mentally deficients, $2 \mathrm{I} 4$

Magnesia and magnesium sulphate, 334

Major, D. R., quoted, $57,60,62,77,81$

Malformations. See also DEFECTs, CONGENITAL

- production of, 6

Malingering, 272

Malnutrition (see also WASTING), 35,253 , $33 \mathrm{I}$

Manual work, 89

- training, I 73, I 75

- - for mentally deficients, 2I 2, 2 I 4

Marasmus. See WASTING

Marginal blepharitis, I 36

Mastoid disease, deaths from, in Evelina Hospital, 234, 235, 238

- - pain from, 300

Masturbation by mentally deficients, 2 I 2

Meals, free, in schools, IO2

Measles, epidemics of, in schools, I 6

- German, seasonal prevalence of, 295

- glands enlarged after, I I4

- idiocy following, 270

- infant mortality from, 230

- loss of appetite after, 276

- seasonal prevalence of, 295

- spread of, by schools, I 12

- wasting after, 275

Meat in school dietary, 103

Medical inspection of boarding-schools, I 49

Medical inspection of school-childrenAnthropometric, 135

Eyes and ears, I 36

Frequency of, I 30

intention of, I $3 \mathrm{I}$

Law relative to, I 28 
Medical inspection of school-chiluren, contd.-

Mental capacity, I 36

Methods of, I 34

Official scheme of, I 29

- schedule of, I 38, I 40

Reports, annual, 140

Requisites for, I 34

Routine of, 135

Speech, I 37

Teeth, I 37

Throat, 137

Medical officer, school. See ScHOOL DOCTOR

- - (Education) London County Council, report of, on dental caries, I 4 I

Meeh quoted, 47

Memory, 58, 63, 7o, 80

- differences between infant and adult, 70

- training of, 157, I 73, I 75

Meningeal hæmorrhage, idiocy from, 200

Meningitis, appearance of case of, 298

- cerebro-spinal, seasonal prevalence of, 295.

- constipation in, 3 ro

- convulsions in, 277

- cry in, 284

- diagnosis of, 299

- effect of, on pulse, 306

- - of, on respiration, 307

- infant mortality from, 228, 229, 235

- - - in Evelina Hospital, 234, 235

- post-basal, age-incidence of, 269

- - idiocy from, 201

- relative frequency of, 296

- tuberculous, age-incidence of, 269

- - moral insanity from, 216

- vomiting in, 309

- - and constipation in, 3 IO

Menstruation, 55

Mental. See also BraIN, CEREBral

- activity, when greatest during day, 106

- attitude of children towards disease, 272

- deficiency, acquired, I96

- - artificial, I 37

- - causes of, 194

- classification of, 195, 210

- congenital, Ig6

- - environment and, I94

- - injury at birth causing, 15

- - medical inspection for, 136

- natural, 137

Mentally deficient children. See also IDIOTS, IMBECILES.
Mentally deficient children-

Admission to workhouses, I 88, 192

Combined defects, 208

Criminal, I92

Guardianship, I 92

Inebriate, 193,196

London County Council, I 88

Metropolitan Asylums Board, I 88

Number in England and Wales, I93

- on school registers, I89, 193

Pauper, 193

Poor law relief, I 88

Private homes, 208

Public control, I87

- - age limit for, 188 , I90

Royal Commission on, 190

Training of, 206, 2 I I

Mentally deficient women, illegitimacy and, 212

Mercurial poisoning, intra-nterine, death from, 8

Mercurials, 320

Mericismus, 4

Metabolism, physiology of, 40

Metropolitan Asylums Board, mentally deficients and, I 88

Microcephalic idiots, 197

- - surgical treatment of, 203

Micturition, frequency of, in infants, 39

Mid-point of body, 24

Midwives, infant mortality and, 247

Migraine, vomiting in, 309

Milk, ass's, 335

— condensed, 335

- - diarrhœa from, 256

- malnutrition from, 253

- cow's, amount of, required by infant, $j \mathrm{I}$, $52,326,329$

- - caloric value of, $5 \mathrm{I}$

- - citrated, 336

- composition of, 5 I

- - diarrhœa from, 256

- - diluents of, 332

- - diseases from, 253,256

- - dried, 335

- - feeds. See INFANT-FEEdING

- pasteurisation of, 334

- - pathogenic, 334

- - peptonised, 335

- - preservatives in, 334

- - storage of, 256,334

- - substitutes for, 335

- - tuberculous, 257

- - whey, 335

- depôts, municipal, $26 \mathrm{r}$

- goat's, 335

- human. See INFANT-FREDING (natural) 
Milk, pasteurised, 334

- peptonised, 335

- supply of boarding-schools, 96, I 6

- whey, 335

Mimicry, mentally deficients and, 2 I 5

Mitral stenosis, effect of, on growth, 275

Mixtures, medicinal, 323

Molar tooth, eruption of first permanent, I 43

Molluscum contagiosum, I20

Mongolian idiots, I98

Monster siren, 6

Moods. See also Emorions, FeELing

- expression of, 59

Moral imbecile, definition of term, I96

- insanity, 2 I 5

- training of mentally deficients, 2 I I

Morality, early training in, I68

- emotions and, 88

- school dormitories and, 94

- young children's, I 55, I 59, I68

Morphia, 320

Mortality, infant. See INFANT MORTALITY

Mother, effect on child of disease in, 7

Motions, infant's, 36

- examination of, 29I, 332, 333

- in intussusception, 310

Mouse, heat-regulation in new-born, 43

- pulse-rate of, 37

Mouth-breathing, effects of, I 43

- medical inspection and, I 37

- mental deficiency and, I44

- public schoolboys and, I49

Movements, infant, before birth, I3, 23

- - at nine months, 74

- varieties of, $6 \mathrm{I}$

Murmurs, cardiac, diagnosis of, 295

- subclavicular, 3 I 2

Murphy, Sir Shirley, quoted, I I 2

Muscles, growth of, 32

Muscle sense, $58,60,65,68,83$

- in mentally deficients, 213

Muscular action, physiological effects of, 22

- - psychological effects of, 58

- atrophy, primary, 270

- co-ordination. See Co-ORDINATION OF MUSCLES

- exercise, food supply needed in, 52

- twitching, 274

Music, appreciation of, by infants, 65

- - of, by mentally deficients, 213
Music, influence of, in training, 170

Mustard baths, 323

Myopia in school-children, I 24

\section{N}

Name, infant's, a factor in developing ideas of self, 7 I

Nasal feeding, 325

Natal physiology, I4

National Society of Day Nurseries, 263

Natural and artificial stupidity, I 37

Nature study, I 73

Needlework, effect of, on eyesight, I 23

Negation, how expressed in early speech, 84

Neo-malthusianism, 248

Nephritis, acute, relative frequency of, 296

Nervous diseases, moral insanity following, 216

- system, examination of, 289

Neuralgia from dental caries, 142

- from eye-strain, I 23

Neurasthenia, rarity of, in childhood, 267

Neuritis, alcoholic, rarity of, in childhood, 267

- optic, 297

- post-diphtheritic, of diaphragm, 307

Neurosis in childhood, 272, 294

- - bearing of, on diagnosis, 294

Neurotic children, I84

- - school conditions producing, I 85

- idiocy, 197

Newman, Dr, quoted, 255

Newsholme, Dr, quoted, II 7, 255

- and Pakes quoted, I 5

New South Wales, infant mortality in, 227

Night-blindness, 4

- terrors, mouth-breathing and, I43

Nipples, care of, in pregnancy, 327

- of wet-nurse, 328

Niven, Dr, quoted, 255, 257

Nostrils, mouth-breathers', I 43

- movements of, in disease, 307

Notification of Births Act, 247

Nourishment, insufficient maternal, effect of, on child, 9

Nurseries, day, 25 I, 263

Nurses, children's hospital, 3 I 6

- wet, 328

Nutrient en emata, 324, 336

Nutrition, physiology of, 43 
Nutrition, defective. See Malnutrition, WASTING

Nystagmus, medical inspection and, 126

— in idiots, 197

Oatmeal-water, recipe for, 332

Obedience, 86, I 55, I 67

- in mentally deficients, 2 I I

Observation centres for mentally deficients, I9I

Obturator foramen at birth, 25

Occupational diseases, 267

Onomatopœic words, 84

Open-air schools, 148

Ophthalmoscopy, 290

Opium, doses of, 320

- susceptibility of children to, 320

- tincture of, $32 \mathrm{I}$

Optical axis in children, 123

Optic disc, examination of, 290

- neuritis, 297

Os calcis at birth, 25

- - prominence of, 26

Osteo-arthritis, rarity of, in childhood, 267

Otitis, acute, relative frequency of, 296

- - pain from, 300

- chronic (otorrhœa), 297

Ovary, growth of, 32

Overlaying, 279

Overwork, I06, 124, I37

- half-timers and, I 33

- neurotic effects of, 185

\section{$\mathrm{P}$}

Pack, cold, 324

Paget's, Mr Charles, experiments on half. timers, I 34

Pain, abdominal, 301

- clinical examination for, 285,287

- cry of, 300

- diagnostic value of, 300

- expression of, by infants, 300

- fear caused by, 301

- hunger and, 60, 69, 300

- imaginary, 30I

- influence of, in developing ideas of self, 71,82

- location of, in infants, 300

- otitis and, 300

- reaction of children to, 300

- referred, 301

- sense of, at birth, 57,60
Pain, sense of, in idiots, 202

Painting, influence of, 170

Palate, cleft. See Cleft ralate

- of idiots, 200, 205

- of mouth-breathers, 143

Palpation, 286

Pancreas, growth of, 32

Pancreatic juice, physiology of, 35

Paper-weaving for mentaliy deficients, 214

Paræsthesia, 290

Paralysed mentally deficient children, $20 \mathrm{~S}$

Paralysis, cerebral disease causing, 297

- examination for, 290

- idiocy from, 200

- infantile, age-incidence of, 268

- influenza causing, 270

- injury at birth causing, I5

- measles causing, 270

- post-diphtheritic, of diaphragm, 307

- - nasal feeding in, 325

- pseudo-, 289

- pseudo-hypertrophic, 270

- - sex-incidence of, 294

Paraplegic idiots, 200

Paresis, 290

$\overrightarrow{-}$ in intracranial disease, 297

Paris, cantines scolaires of, IO2

Parturition, effects of, on child, I4

Passionate children, 183

Pasteurisation, 334

Patent foods. See Proprietary foods

Pauper, mentally deficients, number of, I93

Peabody buildings, infant mortality in, 224

Pediculosis, I20, I44

Pedley, Mr Denison, quoted, I4I

Pelvis at birth, 25

- changes in, 26

Pembrey, Dr, quoted, 42

Pen for infants, 166

Pepsin, 35

Peptonised milk, how to prepare, 335

Percepts, 58

Percussion, 288

Pericarditis, referred pain in, $30 \mathrm{r}$

- vomiting in, 309

Periosteal hæmorrhages in scurvy, 269

Peritoneum, diarrhœa in diseases of, 3 Io

Peritonitis, constipation in, 3 IO

- effect of, on diaphragm, 307

- tuberculous, abdomen in, 303

- vomiting in, 310

Pernicious anæmia, rarity of, in childhood, 267

Pertussis. See Whooping-cough

Pestalozzi, I6z 
Pfaundler and Schlossmann quoted, 50

Pharmaceutical preparations, 32 I

Phenacetin, 320

Phenazone, 320

Phimosis, I 40

Phonation. See SPEECH

Photograph, behaviour of infant on seeing his, 82

Phthisis. See also Tubercle

- diagnosis of, from pleural effusion, 3 I4

- genetous idiots and, 200

- idiots and, 203

- mongols and, 198

- school-children and, II 7

Phylogenetic influence, 4

Physical degeneration. See DegeneraTION, PHYSICAL

- education of mentally deficients, 2 I 2

- exercises during school, I05

Physician, place of, in education, 2

Physiology, antenatal, 4

- - immaturity and, 246

- natal, I4

- postnatal, 20

- stages in, of childhood, 3

- term, child at, I I

Picture-pricking for mentally deficients, $2 \mathrm{I}_{4}$

Pigeon-breast, examination for, 1 36, 284

- mouth-breathing and, 143

- public schoolboys and, I 49

Pigeon, incident of child and, 84

Pills, 321

Placenta, influence of, 7

Plaiting straws, 87

Plantar arch at birth, 25

- changes in, 26

Play and work, relation between, 108

Pleasure, expression of, by infant, 69

- infant's, on seeing his reflection, 72

Pleural effusion, diagnosis of, 312, 3I 3

- - effect of, on cardiac impulse, 3 I 2

Pleurisy, diaphragmatic movements in, 307

Pleuro-pneumonia, referred pain in, 301

Plumbism. See LEAD-POISONING

Pneumonia, aspect of case of, 298

- auscultatory signs in, 312

- coma in, 277

- convulsions in, 277

- diagnosis of, 299, 313

- epistaxis in, 3 I I

- infant mortality from, 230

- pain, referred, in, $30 \mathrm{I}$

- prevalence, seasonal, of, 295

- relative frequency of, 296

- respiration in, 307

- rigors in, 3 II

- simulation of appendicitis by, $30 \mathrm{I}$
Poor law relief for mentally deficients, I 88

Population, density of, and infant mortality, 224

Posology, 3 I 9

Possetting, 35

Post-basal meningitis. See Meningitis, POST-BASAL

Post-diphtheritic paralysis of diaphragm, 307

- - nasal feeding in, 325

Post-febrile idiocy, 20I

Post-mortem examination of still-born children, 247

Post-pharyngeal abscess, stridor from, 308

Pott's belly-ache, 301

Poultices, 323

Pound for infants, I 66

Powders, how to administer, 322

Præcordial bulging, 284, 302

Precocious children, 184

Pregnancy, hygiene of, and infant mortality, $242,243,245$

"Prelinguistic babblings," 78

Prematurity, infant mortality from, 229, 237

- - - from, in hospitals, 235 -

Preparation, evening, I06

Prescriptions, 3 I9

Preyer quoted, 56, 62, 64, 71, 75, 77, 81, 85

Primary amaurotic idiocy, I99

- infantile atrophy, 237

Prize attendance system, II 3, I 32

Prizes and punishments, 167

Prognosis in acute diseases, 278

- in idiocy, 203

Prolapsus ani, 36

Pronoun, personal, first use of, 85,88

Proportions, bodily, changes in, 23, 24

Proprietary foods, 330

Protein, amount of, in infant's body, 34

- consumption of, by infants and adults, 34

Provision of Meals Act, IO2

Prussia, suicides of children in, 185

Pseudo-hypertrophic paralysis, 270, 294

Pseudo-paralysis, 289

Psychology. See under Emotions; INTELLIGENCE; SELF, CONSCIOUSNESS $\mathrm{OF}$, etc.

Ptosis, medical inspection and, I 36

Puberty, growth in height at, 28

- - in weight at, 29

- health of girls at, 180 
Puberty, mental changes at, $x 76$

- physiology of, 54 .

Public elementary schools, 97

- schools, See also BoARDING-SCHOols

- - physical defects of boys at, 149

Puerile breathing, 3I 2

Pulmonary circulation at birth, I7

- diseases. See also LuNGs, DISEASES OF

- - infant mortality from acute, 228, $229,234,235$

Pulse, examination of, in infants, 29r, 3or

- -rate, abnormal, in infants, 305

- age-variations of, 37

- diagnostic value of, 305

- elephant's, 37

- heat-production and, 37

- mouse's, 37

- rabbit's, 37

Punishment, dread of, a cause of neurosis, 185

- as a factor in child-training, $108,16 \mathrm{r}$, I 67

- moral insanity and, 2 I6

- prizes and, 167

Puppies, heat-regulation in, 43

Pyelitis, acute, rigors in, 3 I I

Pyloric stenosis, congenital, lavage for, 325

Pyrexia. See Temperature

\section{Q}

"Quack," use of word, by child, 84

Quetelet and Altherr quoted, 45

Quinine, 320

Quotient, respiratory, 44

$\mathrm{R}$

Rabbit, pulse-rate of, 37

Radiators in classrooms, 99

Radius in infants, 27

Rash, enema, 325

Rat, respiration-rate of, 38

Reading, training mentally deficients in, 215

Realists, 106

Reasoning, 59, 80, 85

- faculties, training of, 175

Recipe for-

Albumin-water, 325

Arrowroot-water, 333
Recipe for, continued-

Baked flour, 326

Barley-water, 332

Lime-water, 332

Oatmeal-water, 333

Pasteurised milk, 334

Peptonised milk, 335

Rice-water, 332

Saline solution, 335

Sherry-whey, 335

Sugar-solution, 335

Whey, 335

Recognition, 58, 63, 70

Recreation during school-hours, 105

- relation between work and, 108

Rectal injections, 324,336

- - in fever, 324

- medicated, 335

- lavage, 325,334

- temperature, $29 \mathrm{I}$

Rectum, examination of, 290

- fissure of, producing constipation, 310

- loss of control of, 297

Recurrent vomiting, 309

Reflex action before birth, $\mathrm{I}_{3}$

- - after birth, 64, 288

- - in disease, 277

- movements of new-born infant, $6 r$

Reflexes, examination of, 290

- in primary amaurotic idiocy, 199

Religious training of children, 168

Rennin in infant's gastric juice, 35

Respiration, abdominal, 307

- diagnostic value of, 306

- effects of anæsthetics on, 306

- - of cerebral disease on, 307

- - of first, on heart, 16

- - of marasmus on, 308

- of paralysed diaphragm on, 307

- - of pneumonia on, 299

- infant's, inhibited by annoyance, 28I

- irregularity of, in children, 306

- mechanism of first, 15

- thoracic, 307

- -rate, age-variations in, 38

- - of lion, 38

- of rat, 38

- - rhinoceros, 38

- surface-area and, 38

Respiratory centre in new-born mammals, 38

- - stimulation of, at birth, I6

- exchange, 39

- quotient, 44

Rest, importance of, in illness, 3 I 8

- and work in schools, IO4

Restlessness as a sign of pain, 300 
Restlessness in pneumonia, 298

Retina, examination of, 290

Retinal impressions, projection of, by infant, 68

Retro-pharyngeal abscess, a cause of sudden death, 279

Rheumatic fever, age-incidence of, 269

- - endocarditis from, 295

- - medical inspection and, I 37

- - relative frequency of, 296

- - seasonal prevalence of, 295

- - tonsillitis in, 304

Rhinoceros, respiration-rate of, 38

Ribs, beading of, 284

- shape of, in infants, 27

Richet quoted, 39

Rickets, breast-feeding and, 253

- diagnosis of idiocy from, 205

- fœtal, 8

- infant mortality from, 228, 229, 23I, $234,235,238$

- - - from, in Evelina Hospital, 234,

$-\frac{235}{226}$ legitimate and illegitimate from,

- laryngismus stridulus in, 308

- peculiar to childhood, 269

- relative frequency of, 296

- shape of head in, 198,284

Rigidity, muscular, in cerebral disease, 297

- - in primary amaurotic idiocy, 199

Rigors, 3 I I

Ring-worm, I20

- medical inspection for, $\mathrm{I} 36$

- schools, I2I

Robinson's, Dr Louis, experiment, 6I

Romanes quoted, $56,76,77,79,85,87$

Round-shoulders from unsuitable desks, 123

Royal Commission on feeble-minded children, I9o

- - on tuberculosis, 257

Rubbing, therapeutic use of, 323

Rübner quoted, 47

Run, ability to, 82

Rupture. See HERnia

\section{$\mathrm{S}$}

Sacro-vertebral prominence, 25, 26

Sacrum, 25

Salicylic acid, 320, 334

Salicylate, bismuth, 334
Saline solution, recipe for, 335

Saliva, physiology of infant's, 35

Salivary glands, growth of, 32

Salol, 334

Salts, inorganic, amount of, in infant's body, 34

- - - retained for growth, 44

- - in urine, 39

Sanatorium, school, I50

Satiety, expression of, 62

Scabies, I 20

Scapula in infants, 27

Scarlet fever, endocarditis from, 295

- - enlarged glands in, I44

- - epidemics of, in schools, II 6

- - idiocy from, 200, 201

- - infant mortality from, 230

- - spread by schools, I I 2

School attendance, specific fevers and, II 2

- children, common pathological conditions of, 140

- classrooms, 98

- closure, II4, II6

- desks, 99, I22

- diet, IOI

- doctor, annual report of, I4O

- - assistance of school-nurse for, I34

- - attendance of, daily, I 32

- - boarding, 150

- - duties of, I 3 I

- - requisites for, I34

- - weekly visits of, in Sweden, I32

- dormitories, 94

- drainage, 97, I 6

- examinations, 157,175

- games, I09

- heating, 99

- holidays, I07, I 7 I

- home and, relation between, I08, III, 170, 172

- hygiene, 92, IOI

- life, risks and dangers of, I I I

- laundries, 96, I I6

- milk-supply, 96, II6

- nurse, I 30, I 3 I, I 34

- prizes, 167

- punishments, 108, I61, 167

- sanatoriums, 150

- time-table, mental fatigue and, I05

- treats, spread of fevers by, II 3

- ventilation, 98, 122

- water-supply, 96

- work, hours of, I06

Schools, boarding. See BoARDINGSCHOOLS

- girl, I80

- infectious fevers in, 112 
Schools, infectious fevers, closure for, I 4

- - - exclusion of cases of, I I4

- - notification of cases of, 114

- - - prevention of, I I 3

- invalid, I47

- medical inspection of. See MedicaL INSPECTION

- open-air, 148

— public elementary, 97

- site for, 92

- special, for mentally deficient children, I 88 , I 91

- - for physically defective children, 147

- Sunday, spread of infectious fevers by, I 3

Seaside resorts for children, 319

Seasonal incidence of certain diseases, 295

- - of diarrhœa, 254

Seasons, effect of, on growth in height, 29

- - of, on growth in weight, $3 \mathrm{I}$

Secretion, internal, of testes and ovaries, 55

- of milk, 50, 337, 328

Séguin, 206

Self, consciousness of, $67,70,8 \mathrm{I}, 85,88$

Selfishness in childhood, 88,183

Sensation, 57

Senses. See Cold, Heat, Hearing, Muscle, Pain, Sigh'T, SMell, Static, Taste, Touch

Sensory training of infants, 164

Sentences, first use of, 84

- understanding of, 77

Septic conditions, infant mortality in Evelina Hospital from, 234, 235

Sequelæ, frequency of, 278

Sewing, effect of, on eyesight, $\mathrm{I} 23$

- training mentally deficients in, 214

Sex, bearing of, on diagnosis, 294

Sexual crime, mentally deficients and, 212

- physiology, instruction in, I76, 180

Schedule of medical inspection by Board of Education, I 38 , 140

Sherry-whey, 335

Shinn, Miss, quoted, $56,63,87$

Shuttleworth, Dr, quoted, $20 \mathrm{I}$

Sickrooms, school, I 5 I

Sight, defective, among public schoolboys, I 49

- effects of school-life on, I 23

- idiot's, 202

- sense of, in infants, 57, 60, 62, 64
Sight testing, I 36

- training, of mentally deficients, 2 I 3

Sinus, frontal, in infants, 25

Siren monster, 6

Site, school, 93, 97

Sitting, delayed power of, in idiots, 205, 302

- first attempts at, 68

Skeleton, changes in, 23

- deformity of, in disease, 302

- growth of, 32

- - of, in disease, 269

Skin diseases in childhood, 267,269

- - contagious in schools, I20

- growth of, 32

- sensitiveness of infant's, 323

Skipping, 87

Skodaic resonance, 314

Skye-terrier, incident of, 87

Sleep, effect of, on heart-beat, 306

- how influenced by disease, 299

- hours of, for children, 104

- to induce, 323

Slojd for mentally deficients, 2 I 4

Small-pox, infant mortality from, 230

Smell, sense of, 57,62

Smiling (see also LAUGHING), 62, 63

Snelling's test-type, I 34

Sneezing, reflex act of, 6 I

Snoring, mouth-breathing and, I43

Society of Day-Nurseries, National, 263

- Westminster Health, 260

Soil suitable for schools, 93

Somnambulism, 274

Sound. See also Hearing

- localisation of, by infants, 65

Sore throat. See ToNsillitis

Space, appreciation of, by infants, 68

Spasm, muscular, in cerebral disease, 297

Spasmus nutans, seasonal prevalence of, 295

Special schools. See Schools, special

Specific fevers. See FEvers, SPECIFIC

- gravity of blood at puberty, 55

Speech-

Attempted, 63,66

Common errors, 83

Date of earliest, 77

Defective, 145

— in idiots, 205

- medical inspection and, 137

- of microcephalics, 197

Delayed, 302

Difficulties in acquiring, 79

Growth of vocabulary, 83 .

Inarticulate, 69 
Speech, continued-

Inflection of verbs, 88

Influence of, on ideas of self, 85

Mechanism of, 145

Psychological effect of, 59

Stages in development of, 78

Training infants, I 66

- mentally deficients, 214

Use of personal pronoun, 88

Spencer, Herbert, quoted, I 56, I 67

Spinal caries, referred pain in, $30 \mathrm{I}$

- cord, activity of, before birth, I 3

- - chronic diseases of, rare in childhood, 268

- - growth of, 32

Spleen, growth of, 32

Splints in rickets, 318

Sponging, cold, 324

Spontaneous education, I63

"Spoon-feeding," I60

Spraying, therapeutic use of, 323

Sputum, diagnostic value of, 309

- methods of obtaining, $29 \mathrm{I}$

Squinting in idiots, 200, 205

- in infants, 6r, 64

- medical inspection and, I 36

St Vitus's dance. See Chorea

Staffordshire, infant mortality in, 223

Stairs in elementary schools, 98

Stammering, I45

- medical inspection and, 137

Standing, first attempts at, 76

Starch, action of infant's saliva on, 35

- in an infant's diet, 33I

- proprietary foods containing, 330

Static sense in infants, 74

Statute of Prerogatives, 187

Steapsin, 35

Steele quoted, I 79

Stenosis, congenital pyloric, gastric lavage for, 325

Sternum in infants, 27

Stigmata of idiocy, 205

Still, Dr, quoted, 238

Still-born children, autopsies on, 247

Stomach, amniotic fluid in, I 3

- capacity of infants, 53

- displacement of, by deformity of chest, 303

- growth of, 32

- lavage of, 325

- - of, to obtain sputum, 29I

- tube, 325

Stones, incident of child and, 88

Stop-positions in speaking, I 45

Stormont House, 209

Strain, muscular and nervous, in childhood, 267
Stretching, first act of, 6 I

Stridor, causes of, 308

Studies as bedrooms, 95

Stuttering, I 45

Subcutaneous injections, 326

- - risks of, 324

Subpubic angle at birth, 25

Succus entericus, 36

Suckling, 34, 327

- reflex movement of, 61

Sudden death, 279

Sugar. See also CARbohydRATES

- medicinal use of, 32 I

- solution, 335

Suicides of children, I 85

Sully, Professor, quoted, 56, 83, 86, 87

Summer diarrhœa. See DiarrheEA, EPIDEMIC

Sun, incident of child and, 86

Superciliary ridges of infants, 25

Supervision of idiots, 207

Suprarenal. See ADRENAL

Suppository, soap, for infants, 325

Surface-area-

Age-variations in, 47

Body-weight and, 36

Formula for calculating, 46

Heat-loss and, 37, 42

Heat-production and, 42

Pulse-rate and, 37

Respiration-rate and, 38

Respiratory exchange and, 39

Weight variations and, 47

Surgical treatment of idiocy, 203

Sweat, excretion of, 39

Sweden, school doctor in, $\mathrm{I}_{32}$

- crêches in, 264

Sympathy, growth of, in children, 88

- desire for, I6r

Syphilis, acquired, rarity of, in childhood, 267

- congenital, relative frequency of, 297

- maternal, intra-uterine death from, 8

- infant mortality from, 228, 229, 234, 235,237

$-\frac{2}{226}-\frac{1}{2}$ legitimate and illegitimate, from,

Syphilitic epiphysitis, 269

- idiocy, I 97

Syrups, 332

\section{$\mathrm{T}$}

Tables-

Caloric value of food required by infant, 49

Feeds, composition of infant's, 329 
Tables, contimued-

Feeds, number of daily, for infants, 53

Heat-loss in infants, 48

Height of boys and girls, 29

lnfant mortality-

Deaths in each of first five years, 219

- in England and Wales, 22 I

- in Evelina Hospital, 234

- _ - - and England and Wales, 235

Mortality, I85I-I907, 2 I 9

- in each of first five years, 2 I 9

- in trimesters of first year, 220

- in first trimester, $22 \mathrm{I}$

- causes of, 228

- - of, by trimesters, 229

- diarrhœa, urban and rural, 255

- legitimate and illegitimate, 225

_ - London and rural, 225, 227

- - - from certain causes, 226

- rural and urban, 223

- - and town, 223

Talking. See SPEECH

Tannic acid, application of, to nipples, 327

Taste, sense of, in infants, 57,62

Tatham, Dr, quoted, 225,227

Teacher's influence on girls, I 82

Teachers, school, instruction of, in hygiene, etc., I 2 I

- - phthisis among, I I 9

Tears, when first shed from grief, 88

- infant's, 63

Teat, infant's rubber, dangers of, 256

Teeth, caries of. See DENTAL CARIES

- cleaning, $\mathrm{I}_{42}$

- date of eruption of first permanent, I 43

- delayed eruption of, in diagnosis, 205, 302

- medical inspection of, 137

Temperature, age-variations in, 43

- appreciation of, by infants, $57,60,62$

- atmospheric, and food-requirements, 52

- - and epidemic diarrhœa, 254

- classroom, 99

- diagnostic value of, 304

- effect of hot bottle on, 323

- infant's, after birth, 42

- instability of, $43,305,323$

- precautions in registering, 29I

- rectal, 29I

- subnormal, 305

- to reduce excessive, 324

Temporal artery, examination of pulse in, $29 I$

Tendon-reflexes, examination of, 290
Terrier, Skye, incident of, 87

Terror caused by pain, 301

Testis, growth of, 32

- undescended, medical inspection and, I 40

- - in idiots, 205

Tetanus in the newly born, 27 I

Thirst, a factor in developing ideas of self, 70

Thorax, changes in, at birth, I6

- - in, from atmospheric pressure, 27

- deformed, 284, 302, 303

- clubbed fingers from, 303

- - effect of, on viscera, 303, 312

- shape of, in infant, 27

- - of, in adult, 28

- unequal expansion of, 313

Threading beads, 87

Throat. See also TonsiLs

- examination of, 292

- medical inspection of, 137

Thrombosis, cerebral, a cause of idiocy, 201

- - epistaxis in, 3 I I

- - rigors in, $3 \mathrm{I} \mathbf{I}$

- syphilitic, rarity of, in childhood, 267

Throwing movements, 78

Thrush, 27I

Thumb, opposition of, $6 \mathrm{I}, 64,75$

Thymus gland, enlarged, causing sudden death, 279

- fœtal, I3

- growth of, 31, 32

Thyroid gland, cretinism and, 199, 203

- - effects of removal in pregnant dogs, I 3

- - growth of, 31, 32

Tigerstedt quoted, 48

Tinea. See Ring-Worm

Tincture of camphor, compound, dose of, $32 \mathrm{I}$

- of cardamoms, compound, $32 \mathrm{I}$

- of lavender, compound, 321

- of opium, 321

Toad, experimental deformity of, 5

Tobacco-smoke, child's attempt to grasp, 85

Toes, infant's interest in his, $7 \mathrm{I}$

- webbed, 4

Tongue-depressor, use of, 292

- sensations of, in infants, $60,62,67$

Tonics, 321

Tonsillitis, acute, relative frequency of, 296

- recurrent, medical inspection and, I 37

- varieties of, 304 
Tonsils and adenoids, effects of, 143

- - growth of, 40

- - medical inspection and, 137

- - shape of chest with, 302

- - treatment of, I44

- enlarged, diagnostic value of, 303

- - dental caries and, I42

- - examination of, 292

- - relative frequency of, 297

Tooth. See also TeEth

- -brush and dental caries, 142

- - drill, I 43

Touch, sense of, at birth, 60

- - of, in developing ideas of self, $7 \mathrm{I}$

- - of, in idiots, 202

---- training of, $2 \mathrm{I} 3$

Town life, infant mortality and, 222, 223, $225,227,255$

Toxic arhythmia, 305

- idiocy, 197

Toxins, transmission of, to fœtus, 8

Training of children-

Adolescence, 174

Esthetics, 169

Authority, 167

Curiosity, I60

Enthusiasm, I60

Ethics, I68

Habits, I59, 166

Home-life, I 7 I

Infancy, $I 6_{3}$

Leisure, $171, \mathrm{I} 76$

Manual work, 173, 175

Methods, 162

Nature-study, I 73

Punishment, I6I, I67

Religion, 168

Rewards, 167

Schools, I70, I72

Sex, 176

Stages, first, 163

- second, 167

- third, I74

- fourth, I63

Training of girls-

Child-rearing, I8I

Classroom work, 180

Co-education, 177

Domestic economy, I8I

School influences, I82

Training of idiots and mentally deficient children-

General, 2 II

Guardianship, 207

Intellectual, 215

Manual, 2I4

Moral, 2 I I
Training of idiots and mentally deficient children, continued -

Motor, 214

Results, I89, 207, 210

Sensory, $2 \mathrm{I} 3$

Speech, 214

Traumatic acquired idiocy, 201

$\overrightarrow{-}-$ surgical treatment of, 203

Treatment-

Acute illness, 315

Chronic illness, $3 \mathrm{I} 7$

Convalescence, 317

Cretinism, I 99

Debility, 318

Digestive troubles, $33 \mathrm{I}$

Idiocy, 203

Medicinal, 319

Methods, special, 322

- unsuited to children, 323

Preventive, 315

Rest, 3 I8

Symptomatic, 3I 7

Tredgold, Dr, quoted, 209

Tremulousness in mental deficiency, $2 \mathrm{I}_{4}$

Trypsin, 35

Tubera ischii, 25, 26

Tubercle, abdominal, diagnosis of, 3 I I

Cow's milk and, 253, 257

Deaths from, in Evelina Hospital, 234, 238

- - in Hospital for Sick Children, 258

- under 18 months from, 240

Dental caries and, I42

Frequency, relative of, 296, 297

Idiocy and, I94, I98, 200, 203

Infant life and, 252, 256

- mortality from, 228, 229, 234, 235, 238

- - legitimate and illegitimate, from, 226

Meningeal, 216, 269, 299

Prevention of, in schools, II7

Revealed, II 8

School-caretakers and, 120

- children and, II 7

- teachers and, II

Sources of infection by, II 8

Tuberculosis, Royal Commission on, 257

Tumour, cerebral. See Cerrebral TUMOUR

Twitches, 274

Typhoid fever, epistaxis in $3 \mathrm{II}$

- - moral insanity from, 216

- - rigors in, $3 \mathrm{II}$

- - seasonal prevalence of, 295 
U

Ulna in infancy, 27

Uncleanliness, infant life and, 252

Underfed children, IOI, I 33

United States, mentally deficients in, I 92

Untruthfulness, 88 , I 84

Uræmic convulsions, 277

Urea, excretion of, in infants, 39

Uric acid, excretion of, in infants, 39

Urine, composition of, 39

- examination of, 290

- incontinence of, 290,297

- secretion of, 13,38

\section{V}

Vacations. See Holidays

Varicella. See CHICKEN-POX

Varicocele, medical inspection and, I40

Variola. See SMALL-PoX

Ventilation of classrooms, 98, I05

- - effects of bad, I 22

- of dormitories, 95

Ventricles at birth, 18

Verbs, inflection of, 88

Vertebral column, 25

Vesicular murmur, 3 I 2

Vierordt quoted, 32, 42

Vision. See SIGHT

Visitors, health, 260

$\overline{-}$ infant protection, 25 I

Vocabulary, growth of, 83

Vocal expression of feelings, 65,69 , 75

- resonance, how to test, $28 \mathrm{I}$

Voice, flexibility of, in infants, 77

- nasal, in mouth-breathers, 143

Voluntary action, 8I

- - and ideas of self, $7 \mathrm{I}, 82$

- movements, 6r, 67

Vomit, examination of, 291, 332, 333

- character of, in gastric trouble, 333

Vomiting, causes of, 309

- and constipation, 310

- diagnostic value of, 309

- physiological, 35

- recurrent, 309

- relative frequency of, 296

- treatment of, by gastric lavage, 325

$-\stackrel{325}{-}$ of, by nutrient enemata, 336
Vowels, first use of, 63,78

- "physiological," I45
Waldschuler, 148

Walking, delayed power of, 205, 302

- development of, $76,77,82$

- psychological effect of, 59, 77

Waller quoted, 47

Walls, classroom, colour of, 99

Wasting, common causes of, 236

- diseases, official use of term, 228

- heart-disease and, 275

- infant mortality from, 228, 229, 232, $234,235,236$

$-\frac{-}{226}$ legitimate and illegitimate, from,

- relative frequency of, 296

- treatment of, 331, 332

- use of term in death certification, 236

"Washing-out." See LAVAGE

Water, amount of, in infant's body, 34

- consumption of, 44

- supply of boarding-schools, 96, I 16

Watering-places suitable for children, 319

Watering-pot, incident of child and, 85

Wean, when to, 326, 329

Weight at birth, 29, 45

- - as an index of health, 10

- - different ages, 30

- body, absolute increase of, 32

- fallacies in estimating, 30

- - in interpreting, 45

- growth in, 29

- loss of, after birth, 30

- variations, diurnal, in, 31

Westminster Health Society, 260

Wet-nurse, average secretion of milk by, $5 \mathrm{I}$

- - - by, how to estimate, 328

- selection of a, 328

Wet-nursing, 327

Whey, 335

- sherry, 335

Whimpering, 62,78

Whooping-cough, epistaxis in, 3 I I

- infant mortality from, 230, 234, 235

- seasonal prevalence of, 295

- vomiting in, 309

Will, earliest manifestation of, 64

Wiltshire, infant mortality in, $\mathbf{2 2 2}$

Windows, classroom, 99

- dormitory, 95 
Women, employment of married, infant mortality and, $246,25 \mathrm{r}, 262$

Wonder, expression of, by infant, 65,75

- limbs, a cause of, to infants, 7 I

Wood-carving for mentally deficients, 2 I4

Woollen jackets, 323

Words, how learnt by infant, 79

- invention of, by children, 79

- understanding of, 77

Work, effect of maternal, on child, Io

Work and rest in schools, IO4

- and play in schools, 108

- hours of, at different ages, I06

Workhouses, mentally deficients and, r88, 192

Writing, 89

— for mentally deficients, 2 r 5

X-rays, favus and, I2I

- for eign bodies in air-passages and, 308

- ring-worm and, I2I

\section{Y}

Yawning, first act of, $6 \mathrm{I}$

Z

Zymotic diseases. See FEvers, sPECIFIC 
• 
RETURN TO the circulation desk of any University of California Library or to the

Bldg. 400, Richmond Field Station

University of California

Richmond, CA 94804-4698

ALL BOOKS MAY BE RECALLED AFTER 7 DAYS

- 2-month loans may be renewed by calling (510) 642-6753

- 1-year loans may be recharged by bringing books to NRLF

- Renewals and recharges may be made 4 days prior to due date.

\section{DUE AS STAMPED BELOW}

\section{JAN 13200}

RETURNED

FEB $04=$

Canta Crus Jitna' 


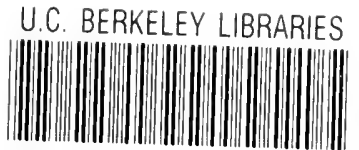

C039709046

\section{UNIVERSITY OF CALIFORNIA LIBRARY}


